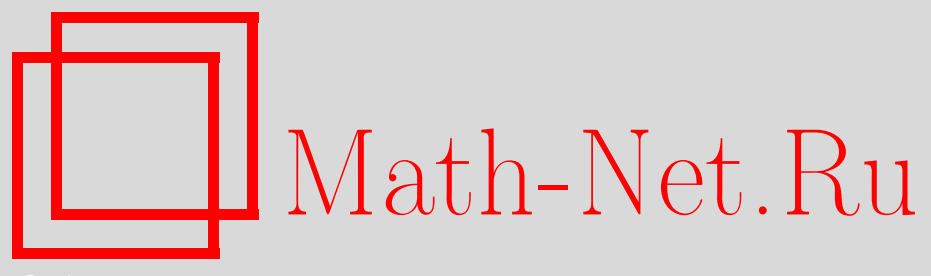

А. А. Герасимов, Д. Р. Лебедев, С. В. Облезин, Новые интегральные представления функций Уиттекера для классических групп Ли, УМН, 2012, том 67, выпуск 1, 396

DOI: https://doi.org/10.4213/rm9463

Использование Общероссийского математического портала Math-Net.Ru подразумевает, что вы прочитали и согласны с пользовательским соглашением http://www . mathnet.ru/rus/agreement

Параметры загрузки:

IP : 107.22 .136 .117

26 апреля 2023 г., 17:52:48

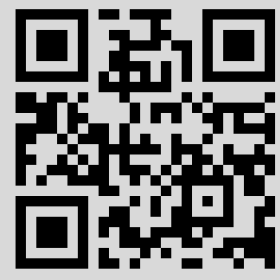




\title{
Новые интегральные представления функций Уиттекера для классических групп Ли
}

\begin{abstract}
А. А. Герасимов, Д. Р. Лебедев, С. В. Облезин
В работе получены новые интегральные представления $\mathfrak{g}$-функций Уиттекера для произвольной полупростой алгебры Ли g. В этих представлениях подынтегральная функция выражается через матричные элементы фундаментальных представлений алгебры g. Для классических алгебр Ли $\mathfrak{s p}_{2 \ell}, \mathfrak{s o}_{2 \ell}$ и $\mathfrak{s o}_{2 \ell+1}$ получена модификация этой конструкции, являющаяся прямым обобщением интегрального представления $\mathfrak{g l}_{\ell+1}$-функций Уиттекера, которое было впервые построено Гивенталем. Представление Гивенталя имеет рекурсивную структуру относительно ранга $\ell+1$ алгебры Ли $\mathfrak{g l}_{\ell+1}$, и предложенное обобщение на все классические алгебры Ли сохраняет это свойство. Ранее было замечено, что интегральный рекурсивный оператор для $\mathfrak{g l}_{\ell+1}$-функции Уиттекера в представлении Гивенталя совпадает с вырождением $\mathscr{Q}$-оператора Бакстера для $\widehat{\mathfrak{g l}}_{\ell+1}$-цепочек Тоды. В этой работе мы строим Q2-операторы для аффинных алгебр Ли $\widehat{\mathfrak{s o}}_{2 \ell}$, $\widehat{\mathfrak{s o}}_{2 \ell+1}$ и скрученной формы алгебры $\widehat{\mathfrak{g l}}_{2 \ell}$. Показано, что связь между рекурсивными интегральными операторами для обобщенных представлений Гивенталя и вырожденными $\mathscr{Q}$-операторами сохраняется для всех классических алгебр Ли.
\end{abstract}

Библиография: 33 названия.

Ключевые слова: функция Уиттекера, цепочка Тоды, оператор Бакстера.

\section{СОДЕРЖАНИЕ}

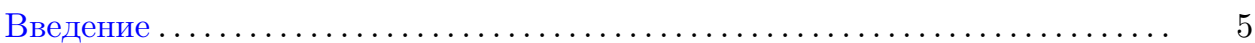

1. Часть $1:$ результаты . . . . . . . . . . . . . . . . . . . . . . . . 7

1.1. Собственные функции цепочки Тоды как матричные элементы 7

1.1.1. Корневые данные для редуктивных групп............. 8

1.1.2. Модель Уиттекера для представлений основной серии ..... 10

1.1.3. Функции Уиттекера как волновые функции цепочек Тоды . 12

1.2. Интегральные представления собственных функций $\mathfrak{g l}_{\ell+1^{-}}$и

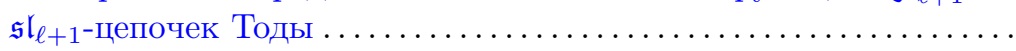

1.2.1. $\mathfrak{g l}_{\ell+1}$-функция Уиттекера: факторизационная параметриза-

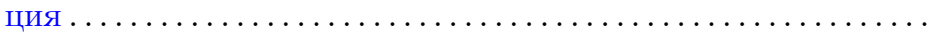

(C) А. А. Герасимов, Д. Р. ЛЕведев, С. В. ОБЛЕзин, 2012 
1.2.2. $\mathfrak{g l}_{\ell+1}$-функция Уиттекера: модифицированная факторизационная параметризация....................... 19

1.2.3. Связь с $\mathscr{Q}$-оператором Бакстера $\widehat{\mathfrak{g l}}_{\ell+1}$-цепочки Тоды .......

1.3. Интегральные представления собственных функций $\mathfrak{s o}_{2 \ell+1}$-цепочки Тоды .

1.3.1. $\mathfrak{s o}_{2 \ell+1}$-функция Уиттекера: факторизационная параметри-

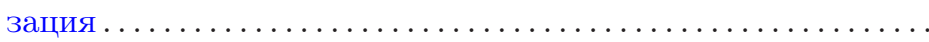

1.3.2. $\mathfrak{s o}_{2 \ell+1}$-функция Уиттекера: модифицированная фактори-

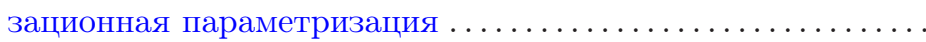

1.3.3. Рекурсия для $\mathfrak{s o}_{2 \ell+1}$-функций Уиттекера и $\mathscr{Q}$-оператор для $B_{\ell}^{(1)}$-цепочки Тоды .

1.4. Интегральные представления собственных функций $\mathfrak{s p}_{2 \ell}$-це-

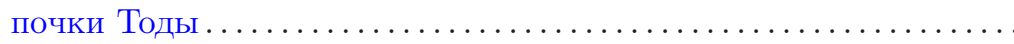

1.4.1. $\mathfrak{s p}_{2 \ell}$-функция Уиттекера: факторизационная параметриза-

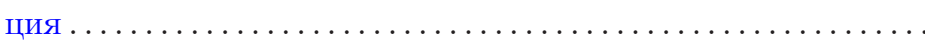

1.4.2. $\mathfrak{s p}_{2 \ell}$-функция Уиттекера: модифицированная факторизаци-

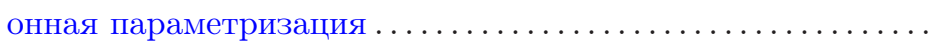

1.4.3. Рекурсия для $\mathfrak{s p}_{2 \ell}$-функций Уиттекера и $\mathscr{Q}$-оператор для $A_{2 \ell-1}^{(2)}$-цепочки Тоды. . . . . . . . . . . . . . . . . . . . .

1.5. Интегральные представления собственных функций $\mathfrak{s o}_{2 \ell}$-цепочки

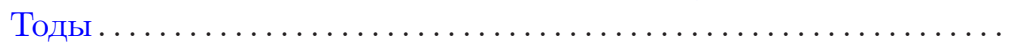

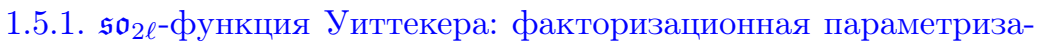

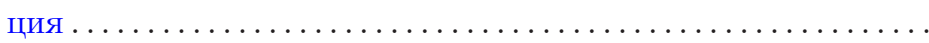

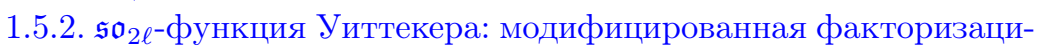

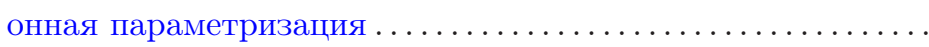

1.5.3. Рекурсия для $\mathfrak{s o}_{2 \ell}$-функций Уиттекера и $\mathscr{Q}$-оператор для

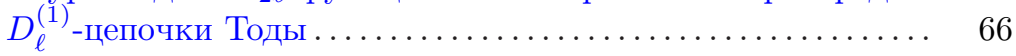

2. Часть 2. Доказательства . . . . . . . . . . . . . . . . . . . . . . . . . . 69

2.1. Мера на $N_{+}$: доказательство леммы $1.2 \ldots \ldots \ldots \ldots \ldots \ldots \ldots \ldots . \ldots 1$

2.2. Векторы Уиттекера для классических групп Ли: доказательства

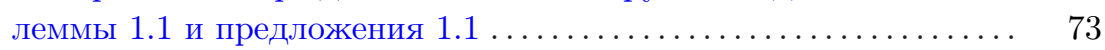

2.3. Явные вычисления для матричных элементов ............ 75

2.3.1. Выражения для $\mathfrak{g l}_{\ell+1}$-матричных элементов: доказательства теоремы 1.1 и теоремы $1.3 \ldots \ldots \ldots \ldots \ldots \ldots \ldots \ldots \ldots$

2.3.2. Выражения для $\mathfrak{s o}_{2 \ell+1}$-матричных элементов: доказательства теоремы 1.4 и теоремы $1.6 \ldots \ldots \ldots \ldots \ldots \ldots \ldots \ldots$

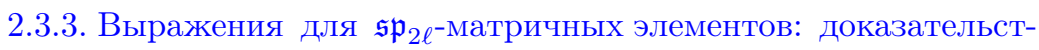
ва теоремы 1.8 и теоремы $1.10 \ldots \ldots \ldots \ldots \ldots \ldots \ldots \ldots \ldots$

2.3.4. Выражения для $\mathfrak{s o}_{2 \ell}$-матричных элементов: доказательства теоремы 1.12 и теоремы $1.14 \ldots \ldots \ldots \ldots \ldots \ldots \ldots \ldots . . \ldots 4$

2.4. Реализация $\mathscr{U}(\mathfrak{g})$ дифференциальными операторами.......... 88

2.4.1. Образующие $\mathfrak{g l}_{\ell+1}$ : доказательство предложения $1.2 \ldots \ldots .90$

2.4.2. Образующие $\mathfrak{s o}_{2 \ell+1}$ : доказательство предложения $1.4 \ldots . .90$

2.4.3. Образующие $\mathfrak{s p}_{2 \ell}$ : доказательство предложения $1.8 \ldots \ldots . .91$

2.4.4. Образующие $\mathfrak{s o}_{2 \ell}$ : доказательство предложения $1.12 \ldots \ldots .93$

Список литературы .................................. 94 


\section{Введение}

В работе [1] А. Гивенталем было предложено замечательное интегральное представление для общих собственных функций гамильтоновых операторов $\mathfrak{g l}_{\ell+1}$-цепочки Тоды (см. также [2]). Это представление естественно возникает в конструкции зеркальной симметрии для топологических замкнутых струн типа $\mathrm{A}$ на $\mathfrak{g l}_{\ell+1}$-многообразиях флагов. Интегральное представление Гивенталя имеет множество интересных свойств. Например, оно обладает явной рекурсивной структурой по отношению к рангу $\ell+1$ соответствующей алгебры Ли $\mathfrak{g l}_{\ell+1}$. Подынтегральная функция в интегральном представлении допускает сугубо комбинаторное описание в терминах простого графа, который можно отождествить с графом Гельфанда-Цейтлина, введенным в работе [3] для описания конечномерных представлений алгебры $\mathfrak{g l}_{\ell+1}$. Такие же графы возникают при изучении плоских вырождений многообразий флагов к горенштейновым торическим многообразиям Фано [4], [5].

В работе [6] интегральное представление Гивенталя изучалось в рамках теоретико-представленческого подхода к теории квантовых интегрируемых систем. Как показал Б. Костант [7], [8], общие собственные функции гамильтоновых операторов g-цепочки Тоды описываются как обобщения классических функций Уиттекера [9] и могут быть выражены в терминах матричных элементов бесконечномерных представлений универсальной обертывающей алгебры $\mathscr{U}(\mathfrak{g})$. В работе [6] показано, что представление Гивенталя для собственных функций $\mathfrak{g l}_{\ell+1}$-цепочки Тоды совпадает с интегральным представлением соответствующего матричного элемента, полученного с использованием параметризации открытого подмножества $\mathfrak{g l}_{\ell+1}$-многообразия флагов.

Концептуальное объяснение данному специальному выбору координат на многообразии флагов можно дать, используя связь с формализмом Q-оператора Бакстера. В [6] также было замечено, что интегральное представление Гивенталя имеет рекурсивную структуру, которая связывает $\mathfrak{g l}_{\ell^{-}}$и $\mathfrak{g l}_{\ell+1}$-функции Уиттекера простым интегральным преобразованием. Соответствующий интегральный оператор совпадает с некоторым вырождением $\mathscr{Q}$-оператора Бакстера для $\widehat{\mathfrak{g l}}_{\ell+1}$-цепочки Тоды [10]. Хорошо известно, что $\mathscr{Q}$-операторы реализуют в квантовых интегрируемых системах квантовые преобразования Бэклунда [11]. В классическом пределе формализм $\mathscr{Q}$-оператора позволяет определить специальную систему координат на фазовом пространстве $\widehat{\mathfrak{g l}}_{\ell+1}$-цепочки Тоды. Тем самым вырожденные $\mathscr{Q}$-операторы определяют специальные координаты на открытом подмножестве многообразия флагов $\mathrm{GL}_{\ell+1} / B$ и непосредственно приводят к интегральному представлению Гивенталя для $\mathfrak{g l}_{\ell+1}$-функции Уиттекера.

До настоящего момента не было известно обобщение интегрального представления Гивенталя для $\mathfrak{g l}_{\ell+1}$-функций Уиттекера на алгебры Ли, отличные от $\mathfrak{g l}_{\ell+1}$. Единственное известное обобщение [5], [12] конструкции Гивенталя состояло в интегральном представлении общих собственных функций определенных вырождений $\mathfrak{g l}_{\ell+1}$-цепочек Тоды [13]. Оно основано на плоских вырождениях многообразий частичных флагов $G / P$ для $G=\mathrm{GL}(\ell+1, \mathbb{C})$, где $P$ параболическая подгруппа в $G[5]$. 
В настоящей работе мы предлагаем универсальную конструкцию интегрального представления $\mathfrak{g}$-функции Уиттекера для произвольной полупростой алгебры Ли g. Для классических алгебр Ли $\mathfrak{s p}_{2 \ell}, \mathfrak{s o}_{2 \ell}$ и $\mathfrak{s o}_{2 \ell+1}$ нам удалось модифицировать эту конструкцию и получить обобщение конструкции Гивенталя для классических алгебр Ли. Полученные таким образом интегральные представления обладают всеми характеристическими свойствами исходного интегрального представления Гивенталя. Интегральные представления для классических алгебр Ли имеют рекурсивную структуру. Далее, подынтегральные выражения в интегральных представлениях допускают комбинаторное описание в терминах графов. Предложенное обобщение на классические алгебры Ли основано на модификации факторизованного представления типичных элементов максимальных унипотентных подгрупп в соответствующих группах Ли [14] (см. также [15], [16]). Конструкция модифицированного факторизованного представления существенно использует реализацию максимальных унипотентных подгрупп классических групп Ли в виде явно заданных подгрупп в группе верхнетреугольных матриц (см., например, [17]). Также мы вводим Q-операторы Бакстера, связанные с классическими аффинными алгебрами Ли $\widehat{\mathfrak{s o}}_{2 \ell}, \widehat{\mathfrak{s o}}_{2 \ell+1}$ и со скрученной формой алгебры $\widehat{\mathfrak{g l}}_{2 \ell}$. Мы показываем, что связь между интегральными операторами рекурсии обобщенного представления Гивенталя и вырожденными $\mathscr{Q}$-операторами сохраняется для всех классических алгебр Ли.

Новое свойство построенного интегрального представления состоит в том, что в отличие от случая $\mathfrak{g l}_{\ell+1}$ (где ядро оператора рекурсии задается простой функцией), интегральные ядра операторов рекурсии для всех других классических групп Ли заданы нетривиальными интегралами. Это наталкивает на мысль о том, что операторы рекурсии можно получить как композиции элементарных операторов. В самом деле, для нулевых собственных значений операторы рекурсии, связывающие собственные функции цепочки Тоды алгебр Ли с соседними рангами, можно представить как композиции элементарных операторов рекурсии, связывающих собственные функции цепочек Тоды различных классических серий.

Подчеркнем, что конструкция интегральных представлений g-функций Уиттекера, изложенная в данной статье, допускает естественную интерпретацию в терминах торификации многообразий флагов для классических групп Ли. Граф, определяющий представление Гивенталя для классической группы Ли, можно отождествить с графом Гельфанда-Цейтлина (см., например, [18]). Эти графы также явно описывают торические вырождения соответствующих многообразий флагов, тем самым обобщая результаты работы [5] на все классические группы Ли.

Интегральное представление типа Гивенталя g-функций Уиттекера для классических алгебр Ли находит прямое приложение в конструкции зеркальной симметрии для замкнутых струн типа А на многообразиях флагов, ассоциированных с классическими группами Ли $G, \mathfrak{g}=\operatorname{Lie}(G)$. Как показано у Гивенталя [1], зеркально симметричными к топологическим теориям струн типа А на многообразиях флагов, ассоциированных с группами Ли $G$, должны быть модели Ландау-Гинзбурга, связанные с двойственными по Ленглендсу группа- 
ми $G^{\vee}$, для которых производящая функция корреляторов нулевого рода есть $\mathfrak{g}^{\vee}$-функция Уиттекера, $\mathfrak{g}^{\vee}=\operatorname{Lie}\left(G^{\vee}\right)$. В случае $\mathfrak{g}=\mathfrak{g l}_{\ell+1}$ Гивенталь нашел описание суперпотенциала двойственной модели Ландау-Гинзбурга в терминах подынтегрального выражения в интегральном представлении соответствующей функции Уиттекера. Более того, интерпретация подынтегральных выражений в интегральных представлениях $\mathfrak{g l}_{\ell+1}$-функции Уиттекера в терминах торических вырождений многообразий флагов [4], [5], [19] позволяет дать явную конструкцию зеркальной симметрии. Мы предполагаем, что подынтегральные функции в интегральных представлениях для функций Уиттекера классических алгебр Ли имеют аналогичную связь с суперпотенциалами двойственных моделей Ландау-Гинзбурга.

Мы следуем следующему плану изложения. В части 1 мы предлагаем новые интегральные представления для $\mathfrak{g}$-функций Уиттекера, отвечающих произвольной полупростой алгебре Ли $\mathfrak{g}$; при этом подынтегральные функции предложенных интегральных представлений выражены через матричные элементы фундаментальных представлений алгебры g. Соответствующее утверждение приведено в предложении 1.1. Затем для классических алгебр Ли $\mathfrak{s o}_{2 \ell+1}, \mathfrak{s p}_{2 \ell}$ и $\mathfrak{s o}_{2 \ell}$ мы получаем модификацию этой конструкции, которая приводит к прямому обобщению интегрального представления для $\mathfrak{g l}_{\ell+1}$-функции Уиттекера, впервые полученного Гивенталем. Соответствующие результаты сформулированы в теоремах 1.3, 1.6, 1.10 и 1.14 соответственно. В части 2 собраны доказательства результатов, сформулированных в части 1 .

Результаты этой работы были анонсированы в [20] (см. также [21], [22]) и в течение последних четырех лет докладывались на многих конференциях.

Авторы благодарны С. Харчеву за обсуждения на начальном этапе этого проекта. Работа первого автора выполнена при поддержке Enterprise Ireland Basic Research Grant. Второй автор благодарен факультету математики Тринити-колледжа в Дублине и Математическому институту им. Макса Планка за гостеприимство. Третий автор также благодарит Математический институт им. Макса Планка за гостеприимство; его исследования были частично поддержаны грантом Пьера Делиня, основанным на премии Бальзана по математике 2004 года.

\section{1. Часть 1: результаты}

\section{1. Собственные функции цепочки Тоды как матричные эле-} менты. Собственные функции $\mathfrak{g}$-цепочки Тоды задаются определенными матричными элементами бесконечномерных представлений алгебры Ли g [7], [8] (подробное изложение этого вопроса можно найти, например, в [23]). В этом разделе мы строим интегральные представления этих матричных элементов, подынтегральные функции которых выражены через матричные элементы конечномерных представлений алгебры Ли g. Затем, в разделе 1.2 и в последующих разделах, получены явные выражения для соответствующих матричных элементов конечномерных представлений, использующих специальную параметризацию на максимальных унипотентных подгруппах. Также получены 
интегральные представления для собственных функций $\mathfrak{g}$-цепочек Тоды, обобщающие результаты Гивенталя для случая $\mathfrak{g}=\mathfrak{g l}_{\ell+1}$ на остальные классические алгебры Ли. Начнем со стандартных определений теории алгебр Ли, следуя в основном книге [24]; обсуждение корневых данных для редуктивных групп также можно найти в обзоре [25].

1.1.1. Корневые данные для редуктивных групп. Корневые данные - это четверка $\left(X, \Phi, X^{\vee}, \Phi^{\vee}\right)$, где $X$ - решетка конечного ранга, $X^{\vee}$ - двойственная решетка, $\Phi$ и $\Phi^{\vee}$ - подмножества в $X$ и $X^{\vee}$, снабженные биекцией $\alpha \mapsto \alpha^{\vee}$ из $\Phi$ в $\Phi^{\vee}$, для которой выполнены следующие условия. Во-первых, $\left\langle\alpha, \alpha^{\vee}\right\rangle=2$ для любого $\alpha \in \Phi$. Во-вторых, подмножества $\Phi$ и $\Phi^{\vee}$ должны быть инвариантны относительно всех автоморфизмов $s_{\alpha}, s_{\alpha}$ :

$$
s_{\alpha}(x)=x-\left\langle x, \alpha^{\vee}\right\rangle \alpha, \quad s_{\alpha} \vee(y)=y-\langle y, \alpha\rangle \alpha^{\vee}, \quad x \in X, \quad y \in X^{\vee}, \quad \alpha \in \Phi .
$$

Пусть $Q \subset X$ - подрешетка, порожденная элементами $\Phi$, и $P$ - решетка, определенная как

$$
P=\left\{x \in X \otimes \mathbb{Q} \mid\left\langle x, \alpha^{\vee}\right\rangle \in \mathbb{Z}, \alpha \in \Phi\right\} .
$$

Тогда $Q \subset P$ и группа $P / Q$ конечна. Пусть $X_{0} \subset X-$ подрешетка, заданная условиями

$$
X_{0}=\left\{x \in X \mid\langle x, y\rangle=0, y \in \Phi^{\vee}\right\} .
$$

С каждой редуктивной группой Ли можно связать корневые данные. А именно, пусть $G$ - связная комплексная редуктивная группа Ли и $H \subset G$ - максимальный тор (подгруппа Картана). Свяжем с парой $(G, H)$ корневые данные $\left(X, \Phi, X^{\vee}, \Phi^{\vee}\right)$ следующим образом. Пусть $X$ - решетка характеров подгруппы $H$, являющаяся свободной абелевой группой конечного ранга, и $X^{\vee}=$ $\operatorname{Hom}\left(\mathbb{C}^{*}, H\right)$ - двойственная решетка однопараметрических мультипликативных подгрупп в $H$. Спаривание $\langle\cdot, \cdot\rangle: X \times X^{\vee} \rightarrow \mathbb{Z}$ задается условием

$$
\lambda(u(t))=t^{\langle\lambda, u\rangle}, \quad \lambda \in X, \quad u \in X^{\vee}, \quad t \in \mathbb{C}^{*} .
$$

Определим конечные подмножества $\Phi$ и $\Phi^{\vee}$ в $X$ и $X^{\vee}$ соответственно, вместе с биекцией $\alpha \mapsto \alpha^{\vee}$ из $\Phi$ в $\Phi^{\vee}$. Присоединенное действие подгруппы $H$ на алгебре Ли $\mathfrak{g}=\operatorname{Lie}(G)$ определяет корневое разложение

$$
\mathfrak{g}=\mathfrak{h} \oplus \sum_{\alpha \in \Phi} \mathbb{C} e_{\alpha}, \quad \mathfrak{h}=\operatorname{Lie}(H),
$$

которое в свою очередь задает подмножество $\Phi \subset X$. Пусть $B$ - борелевская подгруппа, содержащая $H$. На $\Phi$ имеется ровно одно упорядочение $>$, для которого подалгебра $\mathfrak{b}=\operatorname{Lie}(B)$ порождается $\mathfrak{h}=\operatorname{Lie}(H)$ и $e_{\alpha}$ по всем $\alpha>0$. Зафиксируем базис $\Pi=\left\{\alpha_{i}, i \in \Gamma\right\}$ в $\Phi$, согласованный с этим упорядочением, для некоторого множества $\Gamma$.

Имеется разложение $G=Z_{0} \cdot G^{\prime}$, где $Z_{0}$ - связная компонента единицы центра $Z$ группы $G$, а подгруппа $G^{\prime}$ полупроста и совпадает с коммутантом группы $G$. Тогда $H=Z_{0} \cdot H^{\prime}$, где $H^{\prime}$ - максимальный тор в группе $G^{\prime}$. Корневые данные, связанные с парой $\left(G^{\prime}, H^{\prime}\right)$, - это четверка $\left(X / X_{0}, \Phi, Q^{\vee}, \Phi^{\vee}\right)$, где $Q \subset$ $X / X_{0}$. Имея базис $\left\{\alpha_{i}^{\vee}, i \in \Gamma\right\}$ в $Q^{\vee}$ и базис $\left\{\omega_{j}, j \in \widetilde{\Gamma}\right\}$ в $X$, можно выбрать 
такой набор представителей $\left\{\omega_{i}^{\prime}=\omega_{i}+X_{0}, i \in \Gamma \subset \widetilde{\Gamma}\right\}$ в $X / X_{0}$, что $\left\{\omega_{i}^{\prime}, i \in \Gamma\right\}$ образуют базис, двойственный к $\left\{\alpha_{i}^{\vee}, i \in \Gamma\right\}$.

В дальнейшем, если не оговорено противное, мы считаем, что $\mathfrak{g}$ - полупростая алгебра Ли ранга $\ell$. Пусть $\mathfrak{h} \subset \mathfrak{g}-$ картановская подалгебра, и $\mathfrak{b}_{ \pm}-$ пара противоположных борелевских подалгебр алгебры $\mathfrak{g}$, содержащих $\mathfrak{h}$ и согласованных с корневым разложением и с упорядочением $>$ на $\Phi$. Имеется разложение $\mathfrak{g}=\mathfrak{n}_{-} \oplus \mathfrak{h} \oplus \mathfrak{n}_{+}$, где $\mathfrak{n}_{ \pm} \subset \mathfrak{b}_{ \pm}-$нильпотентный радикал. Далее, $\Gamma$ - множество вершин диаграммы Дынкина, отвечающей системе корней полупрострой алгебры $\mathfrak{g}$, и $|\Gamma|=\operatorname{dim} \mathfrak{h}=\ell$. Пусть $\Pi=\left\{\alpha_{i} \in \mathfrak{h}^{*}, i \in \Gamma\right\}-$ множество простых корней, $\left\{\omega_{i} \in \mathfrak{h}^{*}, i \in \Gamma\right\}$ - набор фундаментальных весов и $\Pi^{\vee}=\left\{\alpha_{i}^{\vee} \in \mathfrak{h}, i \in \Gamma\right\}-$ множество кокорней, определенное формулой $\left\langle\alpha_{i}^{\vee}, \omega_{j}\right\rangle=\delta_{i j}$. Рассмотрим матрицу Картана $A=\left\|a_{i j}\right\|, i, j=1, \ldots, \ell$, алгебры Ли $\mathfrak{g}$, элементы которой заданы как $a_{i j}=\left\langle\alpha_{i}^{\vee}, \alpha_{j}\right\rangle$. Обозначим через $R_{+}$множество положительных корней алгебры Ли $\mathfrak{g}$ и пусть $\rho$ - это полусумма положительных корней, т. е. $\rho=\frac{1}{2} \sum_{\alpha \in R_{+}} \alpha$. Найдутся положительные рациональные числа $d_{1}, \ldots, d_{\ell}$, для которых матрица $\left\|b_{i j}\right\|=\left\|d_{i} a_{i j}\right\|$ симметрична. Определим симметрическую билинейную форму на $\mathfrak{h}^{*}$ по формуле $\left(\alpha_{i}, \alpha_{j}\right)=b_{i j}$. Эта форма определяет невырожденное спаривание $\nu: \mathfrak{h} \widetilde{\rightrightarrows} \mathfrak{h}^{*}$, заданное условием $\nu\left(\alpha_{i}^{\vee}\right)=d_{i}^{-1} \alpha_{i}$.

Обозначим через $e_{i}, f_{i}, h_{i}, i=1, \ldots, \ell$, множество образующих Шевалле полупростой алгебры Ли $\mathfrak{g}$, т. е. $e_{i}=e_{-\alpha_{i}}, f_{i}=e_{\alpha_{i}}, h_{i}=\alpha^{\vee}, i=1, \ldots, \ell$. Они удовлетворяют следующим соотношениям:

$$
\begin{gathered}
{\left[h_{i}, h_{j}\right]=0,} \\
{\left[h_{i}, e_{j}\right]=a_{i j} e_{j}, \quad\left[h_{i}, f_{j}\right]=-a_{i j} f_{j}, \quad\left[e_{i}, f_{j}\right]=\delta_{i j} h_{i},} \\
\left(\operatorname{ad} e_{i}\right)^{1-a_{i j}} e_{j}=0, \quad\left(\operatorname{ad} f_{i}\right)^{1-a_{i j}} f_{j}=0, \quad i \neq j .
\end{gathered}
$$

Инвариантная симметрическая билинейная форма на алгебре $\mathfrak{g}$ определяется условиями

$$
\left(h_{i}, h_{j}\right)=b_{i j} d_{i}^{-1} d_{j}^{-1}, \quad\left(e_{i}, f_{j}\right)=\delta_{i j} d_{i}^{-1}, \quad\left(e_{i}, h_{j}\right)=\left(f_{i}, h_{j}\right)=0 .
$$

Единственная неполупростая редуктивная алгебра Ли, рассматриваемая в этой работе, - это алгебра Ли $\mathfrak{g l}_{\ell+1}$. Мы явно задаем алгебру Ли образующими и соотношениями следующим образом. Рассмотрим множество образующих

$$
\left\{e_{i, i \pm 1}, i=1, \ldots, \ell ; \quad e_{k, k}, k=1, \ldots, \ell+1\right\}
$$

алгебры $\mathfrak{g l}_{\ell+1}$. Они удовлетворяют следующим соотношениям:

$$
\begin{aligned}
& {\left[e_{i, i}, e_{j, j}\right]=0,} \\
& {\left[e_{i, i+1}, e_{i+1, i}\right]=e_{i, i}-e_{i+1, i+1},} \\
& {\left[e_{i, i}, e_{i, i+1}\right]=e_{i, i+1} \text {, }} \\
& {\left[e_{i+1, i+1}, e_{i, i+1}\right]=-e_{i, i+1},} \\
& {\left[e_{i, i}, e_{i+1, i}\right]=-e_{i+1, i}} \\
& {\left[e_{i+1, i+1}, e_{i+1, i}\right]=e_{i+1, i},} \\
& \left(\operatorname{ad}_{e_{i, i+1}}\right)^{2} e_{j, j+1}=0, \quad\left(\operatorname{ad}_{e_{i+1, i}}\right)^{2} e_{j+1, j}=0, \quad|i-j|=1 .
\end{aligned}
$$


Пусть $W=W(\mathfrak{g})$ - группа Вейля системы корней алгебры Ли $\mathfrak{g}$. Она порождена простыми отражениями $s_{i}=s_{\alpha_{i}}, i \in \Gamma$, которые действуют в пространстве $\mathfrak{h}^{*}$ посредством следующих линейных преобразований:

$$
s_{i}(\lambda)=\lambda-\left\langle\lambda, \alpha_{i}^{\vee}\right\rangle \alpha_{i}, \quad \lambda \in \mathfrak{h}^{*} .
$$

Определяющие соотношения в группе Вейля имеют вид

$$
s_{i}^{2}=1, \quad\left(s_{i} s_{j}\right)^{m_{i j}}=1, \quad i, j=1, \ldots, \ell,
$$

где

$$
m_{i j}=2,3,4,6, \infty
$$

для

$$
a_{i j} a_{j i}=0,1,2,3, \geqslant 4
$$

соответственно. Для элемента $w \in W$ приведенное слово - это последовательность индексов $I_{w}=\left(i_{1}, \ldots, i_{l(w)}\right), i_{k} \in \Gamma$, минимальной длины, для которой $w=s_{i_{1}} \cdots s_{i_{l(w)}}$. Целое число $l(w)$ называется длиной элемента $w$. Обозначим через $w_{0}$ единственный элемент максимальной длины и положим $m=l\left(w_{0}\right)$. Ниже мы фиксируем подъем $\dot{w} \in G$ элемента $w \in W$, так что $w(u)=\operatorname{Ad}_{\dot{w}} u$ для всех $u \in \mathfrak{g}$. Для простых отражений $s_{i}$ определим

$$
\dot{s_{i}}=e^{f_{i}} e^{-e_{i}} e^{f_{i}}
$$

а для $w=s_{i_{1}} \cdots s_{i_{l(w)}}$ положим $\dot{w}=\dot{s}_{i_{1}} \cdots \dot{s}_{i_{l(w)}}$. Так определенный $\dot{w}$ не зависит от разложения элемента $w$ в произведение простых отражений (см, например, [24; лемма 3.8]).

1.1.2. Модель Уиттекера для представлений основной серии. Рассмотрим универсальную обертывающую $\mathscr{U}(\mathfrak{g})$ алгебры Ли $\mathfrak{g}$. Пусть $V$ и $V^{\prime}-$ это $\mathscr{U}(\mathfrak{g})$ модули. Они называются сопряженными, если имеется такое невырожденное спаривание $\langle\cdot, \cdot\rangle: V^{\prime} \times V \rightarrow \mathbb{C}$, что $\left\langle v^{\prime}, X v\right\rangle=-\left\langle X v^{\prime}, v\right\rangle$ для всех $v \in V, v^{\prime} \in V^{\prime}$ и $X \in \mathfrak{g}$. Мы предполагаем, что действие картановской подалгебры на $V, V^{\prime}$ может быть проинтегрировано до действия максимального тора.

Пусть $B_{-}=N_{-} H$ и $B_{+}=H N_{+}$- пара противоположных борелевских подгрупп, где $H$ - максимальный тор, и $N_{ \pm}$- пара противоположных максимальных унипотентных подгрупп в $G$. Положим $\mathfrak{h}=\operatorname{Lie}(H)$ и $\mathfrak{n}_{ \pm}=\operatorname{Lie}\left(N_{ \pm}\right)$. Характеры алгебры $\mathfrak{n}_{ \pm}$определяются своими значениями на образующих, отвечающих простым корням. Пусть $\chi_{ \pm}: \mathfrak{n}_{ \pm} \rightarrow \mathbb{C}-$ это характер алгебры $\mathfrak{n}_{ \pm}$, определенный условием $\chi_{+}\left(f_{i}\right):=-1$ (соответственно $\chi_{-}\left(e_{i}\right):=-1$ ) для всех $i=1, \ldots, \ell$. Вектор $\psi_{R} \in V$ называется вектором Уиттекера относительно $\chi_{+}$, если

$$
f_{i} \psi_{R}=\chi_{+}\left(f_{i}\right) \psi_{R}=-\psi_{R}, \quad i=1, \ldots, \ell .
$$

Аналогично, вектор $\psi_{L} \in V^{\prime}$ называется вектором Уиттекера относительно $\chi_{-}$, если

$$
e_{i} \psi_{L}=\chi_{-}\left(e_{i}\right) \psi_{L}=-\psi_{L}, \quad i=1, \ldots, \ell
$$


Вектор Уиттекера $\psi$ называется циклическим, если $\mathscr{U}(\mathfrak{g}) \psi=V$. Далее, $\mathscr{U}(\mathfrak{g})$-модуль $V$ называется модулем Уиттекера, если он содержит циклический вектор Уиттекера. Каждый $\mathscr{U}(\mathfrak{g})$-модуль Уиттекера $V$ допускает инфинитезимальный характер $\xi$. Это означает, что имеется такой гомоморфизм $\xi: \mathscr{Z}(\mathfrak{g}) \rightarrow \mathbb{C}$ из центра $\mathscr{Z}(\mathfrak{g}) \subset \mathscr{U}(\mathfrak{g})$, что $z v=\xi(z) v$ для всех $z \in \mathscr{Z}(\mathfrak{g})$ и $v \in V$.

Рассмотрим представление основной серии $\operatorname{Ind}_{B-}^{G} \chi_{\mu}$ группы $G$, индуцированное характером $\chi_{\mu}$ подгруппы $B_{-}=H N_{-}$, который тривиален на $N_{-}$. Оно реализуется в пространстве функций $f \in L^{2}(G)$, удовлетворяющих условию

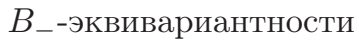

$$
f(b g)=\chi_{\mu}(b) f(g)
$$

где группа $G$ действует правыми сдвигами $\left(g_{1} \cdot f\right)\left(g_{2}\right)=f\left(g_{2} g_{1}\right)$. Мы интересуемся инфинитезимальной формой $\operatorname{Ind}_{\mathscr{U}(\mathfrak{G})}^{\mathscr{U}(\mathfrak{g})} \chi_{\mu}$ этого представления. Действие алгебры Ли $\mathfrak{g}=\operatorname{Lie}(G)$ в инфинитезимальной форме задано формулой

$$
(X f)(g)=\left.\frac{d}{d \varepsilon} f\left(g e^{\varepsilon X}\right)\right|_{\varepsilon \rightarrow 0}, \quad X \in \mathfrak{g} .
$$

Обозначим через $\left(\pi_{\mu}, V_{\mu}\right)$ соответствующий $\mathscr{U}(\mathfrak{g})$-модуль.

Пусть $G(\mathbb{R})$ - вполне расщепимая вещественная форма редуктивной группы Ли $G, \mathfrak{g}_{\mathbb{R}}=\operatorname{Lie}(G(\mathbb{R}))$ - соответствующая алгебра Ли и $N_{+}(\mathbb{R}) \subset N_{+} \cap G(\mathbb{R})$ унипотентная подгруппа в $G(\mathbb{R})$. Рассмотрим биинвариантную меру Хаара $d \mu_{G(\mathbb{R})}$ на $G(\mathbb{R})$. Имеет место разложение Брюа $G(\mathbb{R})=\coprod_{w \in W} B_{-}(\mathbb{R}) \dot{w} B_{+}(\mathbb{R})$. Пусть $G_{0}(\mathbb{R})=B_{-}(\mathbb{R}) N_{+}(\mathbb{R})$ - это $w$-компонента этого разложения при $w=1$. Ограничение меры $d \mu_{G(\mathbb{R})}$ на $G_{0}(\mathbb{R})$ с точностью до нормализации имеет следующий вид (см. [26]):

$$
d \mu_{G(\mathbb{R})}(g)=\delta_{B_{+}(\mathbb{R})}(b) d \mu_{B_{+}(\mathbb{R})}(b) \wedge d \mu_{N_{+}(\mathbb{R})}(x) .
$$

Здесь $\delta_{B_{+}(\mathbb{R})}$ - модулярная функция на $B_{+}(\mathbb{R}) ;$ для любого $b=n g_{0} \in N_{+}(\mathbb{R}) H$ она равна $\delta_{B_{+}(\mathbb{R})}(b)=\exp \left\{2\left\langle\rho, \ln g_{0}\right\rangle\right\}$.

Положим $\mu=i \lambda-\rho$ и рассмотрим невырожденное спаривание $V_{\mu} \times V_{\mu} \rightarrow \mathbb{C}$ :

$$
\left\langle f_{1}, f_{2}\right\rangle=\int_{N_{+}(\mathbb{R})} d \mu_{N_{+}(\mathbb{R})}(x) f_{1}(x) \overline{f_{2}(x)},
$$

где $d \mu_{N_{+}(\mathbb{R})}$ - ограничение $(1.11)$ на $N_{+}(\mathbb{R})$. Это определяет на $V_{\mu}$ структуру унитарного представления $\pi_{\mu}$ алгебры $\mathscr{U}\left(\mathfrak{g}_{\mathbb{R}}\right)$, и мы имеем $\left\langle f_{1}, X f_{2}\right\rangle=$ $-\left\langle X f_{1}, f_{2}\right\rangle$ для любого $X \in \mathfrak{g}_{\mathbb{R}}$.

Мы будем рассматривать несколько более общее спаривание, которое определяется следующим образом. Заметим, что $N_{+}(\mathbb{R}) \subset N_{+}$является вещественной некомпактной подгруппой половинной размерности. Имеется естественная комплексная структура на алгебре Ли $\mathfrak{g}=\mathfrak{g}_{\mathbb{R}} \otimes \mathbb{C}=\mathfrak{g}^{1,0} \oplus \mathfrak{g}^{0,1}$, которая индуцирует комплексную структуру на $N_{+}$. Тогда пространство голоморфных функций на $N_{+}$является модулем относительно голоморфного действия подалгебры $\mathfrak{g}^{1,0} \subset \mathfrak{g}$. Правоинвариантная мера $d \mu_{N_{+}(\mathbb{R})}$ может быть продолжена

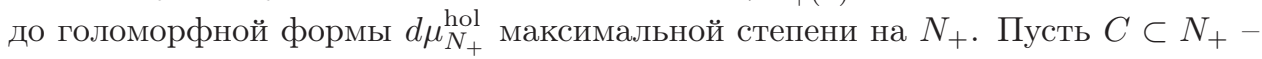


произвольное некомпактное подмногообразие половинной размерности. Рассмотрим спаривание

$$
\left\langle f_{1}, f_{2}\right\rangle_{C}=\int_{C} d \mu_{N_{+}}^{\text {hol }}(x) f_{1}(x) \overline{f_{2}(\bar{x})}
$$

на пространстве $\mathscr{S}_{C}^{\mathrm{hol}}\left(N_{+}\right)$голоморфных функций на $N_{+}$, которые вместе со всеми своими производными экспоненциально убывают после ограничения на $C$. Это спаривание удовлетворяет условиям $\left\langle f_{1}, X f_{2}\right\rangle_{C}=-\left\langle X f_{1}, f_{2}\right\rangle_{C}$ для любого $X \in \mathfrak{g}^{1,0}$.

1.1.3. Функции Уиттекера как волновые функции цепочек Тоды. Следуя Костанту [7], [8], можно выразить собственные функции $\mathfrak{g}$-цепочек Тоды в терминах инвариантного спаривания на модулях Уиттекера:

$$
\Psi_{\lambda}^{\mathfrak{g}}(x)=e^{-\langle\rho, x\rangle}\left\langle\psi_{L}, \pi_{\mu}\left(e^{-h_{x}}\right) \psi_{R}\right\rangle, \quad x \in \mathfrak{h},
$$

где $h_{x}:=\sum_{i=1}^{\ell}\left\langle\omega_{i}, x\right\rangle h_{i}$. В частном случае $\mathfrak{g}=\mathfrak{s l}_{2}$ функция $\Psi_{\lambda}^{\mathfrak{g}}(x), x \in \mathbb{R}$, совпадает с классической модифицированной функцией Бесселя 2-го рода [9] (иногда эту функцию называют функцией Макдональда). Ниже мы используем для матричного элемента (1.12) термин "g-функция Уиттекера" (см., например, [23]). Несколько другое определение функции Уиттекера дано в [27], [28].

Рассмотрим следующее множество коммутирующих дифференциальных операторов $\mathscr{H}_{k} \in \operatorname{Diff}(\mathfrak{h}), k=1, \ldots, \ell$, отвечающих множеству $\left\{c_{k}, k=1, \ldots, \ell\right\}$ образующих центра $\mathscr{Z}(\mathfrak{g}) \subset \mathscr{U}(\mathfrak{g})$ :

$$
\mathscr{H}_{k} \Psi_{\lambda}^{\mathfrak{g}}(x)=e^{-\langle\rho, x\rangle}\left\langle\psi_{L}, \pi_{\mu}\left(e^{-h_{x}}\right) c_{k} \psi_{R}\right\rangle .
$$

Квадратичный образующий центра $\mathscr{Z}(\mathfrak{g})$ (элемент Казимира) имеет вид

$$
c_{2}=\frac{1}{2} \sum_{i, j=1}^{\ell} c_{i j} h_{i} h_{j}+\frac{1}{2} \sum_{\alpha \in R_{+}}\left(e_{\alpha} f_{\alpha}+f_{\alpha} e_{\alpha}\right)
$$

где матрица $\left\|c_{i j}\right\|=\left\|d_{i} d_{j}\left(b^{-1}\right)_{i j}\right\|$ обратна к матрице $\left\|\left(\alpha_{i}^{\vee}, \alpha_{j}^{\vee}\right)\right\|$. Пусть $\left\{\epsilon_{i}, i=\right.$ $1, \ldots, \ell\}$ - ортонормированный базис в $\mathfrak{h}$, т. е. $\left(\epsilon_{i}, \epsilon_{j}\right)=\delta_{i j}$, и $x=\sum_{i=1}^{\ell} x_{i} \epsilon_{i}-$ разложение элемента $x \in \mathfrak{h}$ по этому базису. Проекция (1.13) элемента (1.14) определяет хорошо известный гамильтонов оператор g-цепочки Тоды (см., например, [13])

$$
\mathscr{H}_{2}^{\mathfrak{g}}=-\frac{1}{2} \sum_{i=1}^{\ell} \frac{\partial^{2}}{\partial x_{i}^{2}}+\sum_{i=1}^{\ell} d_{i} e^{\left\langle\alpha_{i}, x\right\rangle}
$$

Операторы $\mathscr{H}_{k}$ образуют полный набор коммутирующих гамильтонианов g-цепочки Тоды [7]. 
Отметим, что собственные функции (1.12) g-цепочки Тоды записаны в весьма абстрактной форме. Чтобы получить явные интегральные представления, для начала выразим матричные элементы (1.12) бесконечномерных представлений в терминах матричных элементов конечномерных представлений алгебры $\mathscr{U}(\mathfrak{g})$. Пусть $\pi_{i}, i \in \Gamma$, - фундаментальные представления, отвечающие фундаментальным весам $\omega_{i}, i \in \Gamma$, алгебры Ли $\mathfrak{g}$, и пусть $\xi_{\omega_{i}}^{+/-}, i \in \Gamma$, - старшие/младшие векторы в этих представлениях, которые определяются условиями $\mathfrak{n}_{ \pm} \xi_{i}^{ \pm}=0, i \in \Gamma$, и нормализованы так, что $\left\langle\xi_{\omega_{i}}^{-} \mid \xi_{\omega_{i}}^{+}\right\rangle=1$. Для старшего вектора $\xi_{\omega_{i}}^{+}$в фундаментальном представлении $V_{\omega_{i}}$ имеем $\dot{s}_{i}^{-1} \xi_{\omega_{i}}^{+}=e_{i} \xi_{\omega_{i}}^{+}$. Рассмотрим следующие матричные элементы в фундаментальных (конечномерных) представлениях:

$$
\Delta_{\omega_{i}, \dot{w}}(g)=\left\langle\xi_{\omega_{i}}^{-}\left|\pi_{i}(g) \pi_{i}(\dot{w})\right| \xi_{\omega_{i}}^{+}\right\rangle, \quad w \in W, \quad g \in G .
$$

Лемма 1.1. Левый и правый векторы Уиттекера, определенные в (1.8) и (1.7), имеют вид

$$
\begin{aligned}
& \psi_{R}(v)=\exp \left\{-\sum_{i=1}^{\ell} \frac{\Delta_{\omega_{i}, \dot{s}_{i}^{-1}}(v)}{\Delta_{\omega_{i}, 1}(v)}\right\}, \\
& \psi_{L}(v)=\prod_{i=1}^{\ell}\left(\Delta_{\omega_{i}, \dot{w}_{0}^{-1}}(v)\right)^{\imath\left\langle\lambda, \alpha_{i}^{\vee}\right\rangle-1} \cdot \exp \left\{\sum_{i=1}^{\ell} \frac{\Delta_{\omega_{i}, \dot{w}_{0}^{-1} \dot{s}_{i}^{-1}(v)}}{\Delta_{\omega_{i}, \dot{w}_{0}^{-1}}(v)}\right\} .
\end{aligned}
$$

Доказательство приведено в разделе 2.2 части 2.

ПРЕДЛОЖЕНИЕ 1.1. Пусть $\mathfrak{g}$ - полупростая алгебра Ли. Общие собственнъе функиии (1.12) g-цепочки Тоды допускают следующее интегральное представление:

$$
\begin{aligned}
\Psi_{\lambda}^{\mathfrak{g}}(x)=e^{\imath\langle\lambda, x\rangle} \int_{C} d \mu_{N_{+}}^{\mathrm{hol}}(v) \prod_{i=1}^{\ell}\left(\Delta_{\omega_{i}, \dot{w}_{0}^{-1}}(v)\right)^{\imath\left\langle\lambda, \alpha_{i}^{\vee}\right\rangle-1} \\
\quad \times \exp \left\{\sum_{i=1}^{\ell}\left(\frac{\Delta_{\omega_{i}, \dot{w}_{0}^{-1} \dot{s}_{i}^{-1}(v)}}{\Delta_{\omega_{i}, \dot{w}_{0}^{-1}}(v)}-e^{\left\langle\alpha_{i}, x\right\rangle} \frac{\Delta_{\omega_{i}, \dot{s}_{i}^{-1}}(v)}{\Delta_{\omega_{i}, 1}(v)}\right)\right\},
\end{aligned}
$$

где $\Delta_{\omega_{i}, \dot{w}}(g)$ определено в (1.16). Здесъ $C \subset N_{+}^{-}$некомпактный иикл половинной размерности, для которого подынтегральное выражение экспоненииально убъвает на границе и на бесконечности. Интегральная мера определяется как ограничение на $C$ голоморфного продолжения $d \mu_{N_{+}}^{\text {hol }}$ правоинвариантной меры $d \mu_{N_{+}(\mathbb{R})}$ на $N_{+}(\mathbb{R})$.

Первый пример интегрального представления этого типа для $\mathfrak{g l}_{n}$-функций Уиттекера был рассмотрен в [29]. Указанное выше представление является его прямым обобщением. Доказательство предложения 1.1 дано в разделе 2.2 части 2 .

Выражение (1.19) для функции Уиттекера является более детальным по сравнению с (1.12), однако все равно не дает явного интегрального представления. Для получения приемлемого представления функций Уиттекера надо 
выбрать параметризацию на $N_{+}$(или на его открытом подмножестве) и выразить меру $d \mu_{N_{+}}^{\text {hol }}$ и матричные элементы, входящие в (1.19), через соответствующие координаты на $N_{+}$. Естественный выбор состоит в разложении элементов из открытого подмножества максимальной унипотентной подгруппы произвольной группы Ли в произведение определенного вида [14] (см. также [16], [15]). Для каждого $i \in \Gamma$ рассмотрим однопараметрическую подгруппу $X_{i}(t)=\exp \left\{t f_{i}\right\}$ в $N_{+}$. Фиксируем разложение элемента максимальной длины $w_{0}$ группы Вейля $W=W(\mathfrak{g})$, отвечающее приведенному слову $I_{w_{0}}=\left(i_{1}, \ldots, i_{m}\right), l\left(w_{0}\right)=m=\operatorname{dim} N_{+}$. Тогда отображение

$$
\mathbb{C}^{m} \rightarrow N_{+}^{(0)}, \quad\left(t_{1}, \ldots, t_{m}\right) \longmapsto v\left(t_{1}, \ldots, t_{m}\right)=X_{i_{1}}\left(t_{1}\right) \cdots X_{i_{m}}\left(t_{m}\right),
$$

является бирациональным изоморфизмом. Это определяет параметризацию открытого подмножества $N_{+}^{(0)}$ в $N_{+}$, которую мы называем факторизационной. Параметризации, отвечающие различным выборам приведенного слова $I_{w_{0}}$, связаны бирациональными преобразованиями, которые явно описаны Г. Люстигом [14]. Правоинвариантная мера здесь допускает следующее описание.

Лемма 1.2. Правоинвариантная мера $d \mu_{N_{+}}^{\text {hol }}$ ф факторизационной параметризачии задается формулой

$$
d \mu_{N_{+}}^{\mathrm{hol}}(v)=\prod_{i=1}^{\ell} \Delta_{\omega_{i}, \dot{w}_{0}^{-1}}(v) \bigwedge_{k=1}^{m} \frac{d t_{k}}{t_{k}} .
$$

Доказательство этого факта дано в разделе 2.1 части 2.

Тем самым проблема нахождения явных интегральных представлений для функций Уиттекера в факторизационной параметризации (1.20) сводится к вычислению матричных элементов конечномерных представлений алгебры Ли $\mathfrak{g}$ в этой параметризации. Ниже (в теоремах 1.1, 1.4, 1.8 и 1.12) мы приводим соответствующие интегральные представления функций Уиттекера, а явные вычисления матричных элементов конечномерных представлений приведены в разделе 2.2. Следует отметить, что полученное таким образом интегральное представление для $\mathfrak{g}=\mathfrak{g l}_{\ell+1}$ не совпадает с представлением Гивенталя [1]. Заметим, что для классических серий алгебр Ли факторизационная параметризация (1.20) имеет рекурсивную структуру относительно ранга $\ell$, которая отражает рекурсивную структуру приведенного разложения элемента $w_{0} \in W$. Однако эта структура не дает простой рекурсивной формы для матричных элементов бесконечномерных представлений в факторизационной параметризации и не воспроизводит рекурсивную структуру на интегральном представлении Гивенталя.

В работе [6] предложена модификация факторизационной параметризации (1.20) для $\mathfrak{g}=\mathfrak{g l}_{\ell+1}$. Там показано, что интегральное представление (1.19) в этой параметризации в точности воспроизводит интегральное представление Гивенталя для $\mathfrak{g l}_{\ell+1}$-функций Уиттекера. В частности, для этой параметризации рекурсивная структура приведенного разложения элемента $w_{0} \in W\left(A_{\ell}\right)$ определяет рекурсивную структуру интегрального представления соответствующей функции Уиттекера. 
В данной работе мы обобщаем результаты [6] на все классические серии алгебр Ли. Мы предлагаем модификацию факторизационной параметризации (1.20), основанную на специальной реализации максимальных унипотентных подгрупп $N_{+} \subset G$ классических групп Ли как явно определенных подгрупп в максимальной унипотентной подгруппе общей линейной группы. Для любой простой классической группы Ли максимальная унипотентная подгруппа может быть реализована как подгруппа в группе верхнетреугольных матриц подходящего размера с единицами на диагонали (см., например, [17]). Соответствующее подмножество верхнетреугольных матриц для классической группы Ли можно описать явно. Мы определяем параметризацию на максимальных унипотентных подгруппах классических групп посредством параметризации специального вида на соответствующем подмножестве верхнетреугольных матриц. Используя эту параметризацию, мы получаем явные интегральные представления для функций Уиттекера, связанных с классическими группами, и показываем, что эти интегральные представления обладают всеми характеристическими свойствами интегрального представления Гивенталя для $\mathfrak{g l}_{\ell+1}$-функций Уиттекера. В частности, в новой параметризации интегральное представление для функций Уиттекера обладает рекурсивной структурой по рангу соответствующей алгебры Ли.

1.2. Интегральные представления собственных функций $\mathfrak{g l}_{\ell+1}-\mathbf{и}$ $\mathfrak{s l}_{\ell+1}$-цепочек Тоды. В этом разделе мы напоминаем конструкцию интегральных представлений для собственных функций $\mathfrak{g l}_{\ell+1}-$ и $\mathfrak{s l}_{\ell+1}$-цепочек Тоды, которая использует факторизационную параметризацию (1.20) на открытом подмножестве $N_{+}^{(0)}$ максимальной унипотентной подгруппы $N_{+} \subset \mathrm{GL}(\ell+1)$ и ее модификацию из работы [6]. Вторая параметризация приводит к интегральному представлению, которое ранее было получено Гивенталем [1] исходя из других соображений. Затем эти конструкции обобщаются на g-цепочки Тоды для произвольных классических алгебр Ли $\mathfrak{g}$.

Начнем со случая редуктивной алгебры Ли $\mathfrak{g l}_{\ell+1}$. Пусть $\left\{\epsilon_{1}, \ldots, \epsilon_{\ell+1}\right\}-$ opтонормированный базис в $\mathbb{R}^{\ell+1}$, т. е. $\left(\epsilon_{i}, \epsilon_{j}\right)=\delta_{i, j}$. Корни и фундаментальные веса для $\mathfrak{g l}_{\ell+1}$ отождествляются со следующими векторами в $\mathbb{R}^{\ell+1}$ :

$$
\alpha_{i}=\epsilon_{i+1}-\epsilon_{i}, \quad i=1, \ldots, \ell, \quad \omega_{j}=-\epsilon_{j}, \quad j=1, \ldots, \ell+1 .
$$

Используя спаривание на $\mathbb{R}^{\ell+1}$, кокорни $\alpha_{i}^{\vee}$ можно отождествить с соответствующими корнями $\alpha_{i}$. Этой системе корней/весов отвечает квантовая интегрируемая $\mathfrak{g l}_{\ell+1}$-система Тоды, для которой имеется $(\ell+1)$ попарно коммутирующих функционально независимых квантовых гамильтонианов $\mathscr{H}_{k}^{\mathfrak{g l} l_{\ell+1}}$, $k=1, \ldots, \ell+1$. Мы интересуемся явными интегральными представлениями общих собственных функций полного набора квантовых гамильтоновых операторов для $\mathfrak{g l}_{\ell+1}$. Например, линейный и квадратичный квантовые гамильтонианы $\mathfrak{g l}_{\ell+1}$-цепочки Тоды задаются формулами

$$
\mathscr{H}_{1}^{\mathfrak{g l}_{\ell+1}}=-\imath \sum_{i=1}^{\ell+1} \frac{\partial}{\partial x_{i}},
$$




$$
\mathscr{H}_{2}^{\mathfrak{g l}_{\ell+1}}=-\frac{1}{2} \sum_{i=1}^{\ell+1} \frac{\partial^{2}}{\partial x_{i}^{2}}+\sum_{i=1}^{\ell} e^{x_{i+1}-x_{i}},
$$

и собственная функция должна удовлетворять следующим уравнениям:

$$
\begin{aligned}
& \mathscr{H}_{1}^{\mathfrak{g l}_{\ell+1}}(x) \Psi_{\lambda_{1}, \ldots, \lambda_{\ell+1}}^{\mathfrak{g l}_{\ell+1}}\left(x_{1}, \ldots, x_{\ell+1}\right)=\sum_{i=1}^{\ell+1} \lambda_{i} \Psi_{\lambda_{1}, \ldots, \lambda_{\ell+1}}^{\mathfrak{g l}_{\ell+1}}\left(x_{1}, \ldots, x_{\ell+1}\right), \\
& \mathscr{H}_{2}^{\mathfrak{g l}_{\ell+1}}(x) \Psi_{\lambda_{1}, \ldots, \lambda_{\ell+1}}^{\mathfrak{g l} l_{\ell+1}}\left(x_{1}, \ldots, x_{\ell+1}\right)=\frac{1}{2} \sum_{i=1}^{\ell+1} \lambda_{i}^{2} \Psi_{\lambda_{1}, \ldots, \lambda_{\ell+1}}^{\mathfrak{g l} \mathfrak{l}_{\ell+1}}\left(x_{1}, \ldots, x_{\ell+1}\right) .
\end{aligned}
$$

Общая собственная функция квантовых гамильтонианов допускает следующее представление в виде матричного элемента:

$$
\Psi_{\lambda}^{\mathfrak{g l} \mathfrak{l}_{\ell+1}}(x)=e^{\sum x_{i} \rho_{i}}\left\langle\psi_{L}, \pi_{\mu}\left(e^{\sum x_{i} E_{i, i}}\right) \psi_{R}\right\rangle,
$$

где $\rho_{i}=-(\ell-2 i+2) / 2, i=1, \ldots, \ell+1,-$ компоненты $\rho$ в базисе $\left\{\epsilon_{i}, i=\right.$ $1, \ldots, \ell+1\}$ пространства $\mathbb{R}^{\ell+1}$.

Конструкция для полупростой алгебры Ли $\mathfrak{s l}_{\ell+1}$ вполне аналогична случаю редуктивной алгебры Ли $\mathfrak{g l}_{\ell+1}$. Корни и фундаментальные веса алгебры $\mathfrak{s l}_{\ell+1}$ можно записать в следующем виде (см., например, [30]):

$$
\alpha_{i}=\epsilon_{i+1}-\epsilon_{i}, \quad \omega_{i}=-\left(\epsilon_{1}+\cdots+\epsilon_{i}\right)+\frac{i}{\ell+1}\left(\epsilon_{1}+\cdots+\epsilon_{\ell+1}\right),
$$

где $i=1, \ldots, \ell$. Это представление системы корней/весов $A_{\ell}$ можно получить из системы корней/весов алгебры Ли $\mathfrak{g l}_{\ell+1}$ следующим образом. Выберем двойственный базис из фундаментальных весов для $\mathfrak{g l}_{\ell+1}$ :

$$
\omega_{i}^{\prime}=-\epsilon_{1}-\cdots-\epsilon_{i},
$$

где $\left\langle\omega_{i}^{\prime}, \alpha_{j}^{\vee}\right\rangle=\delta_{i, j}$ при $i, j=1, \ldots, \ell$ и $\left\langle\omega_{\ell+1}^{\prime}, \alpha_{j}^{\vee}\right\rangle=0$ при $j=1, \ldots, \ell$. Тогда $\omega_{\ell+1}^{\prime}$ можно рассматривать как образующий элемент $X_{0}$. Полагая

$$
\omega_{i}=\omega_{i}^{\prime}-\frac{i}{\ell+1} \omega_{\ell+1}^{\prime},
$$

несложно получить множество (1.28) фундаментальных весов для $\mathfrak{s l}_{\ell+1}$.

Свяжем с этими корневыми данными квантовую интегрируемую $\mathfrak{s l}_{\ell+1}$-систему Тоды, которая обладает $\ell$ попарно коммутирующими функционально независимыми гамильтонианами $\mathscr{H}_{k}^{\mathfrak{s l}_{\ell+1}}, k=1, \ldots, \ell$. Здесь удобнее рассматривать гамильтонианы $\mathfrak{s l}_{\ell+1}$-цепочки Тоды как подмножество $\mathscr{H}_{k}^{\mathfrak{g l} \ell+1}, k=2, \ldots, \ell+1$, в множестве гамильтонианов $\mathfrak{g l}_{\ell+1}$-цепочки, действующих на ядре линейного гамильтониана $\mathscr{H}_{1}^{\mathfrak{g l} \mathfrak{l}_{\ell+1}}$. Например, собственная функция квадратичного квантового гамильтониана $\mathfrak{s l}_{\ell+1}$-цепочки Тоды должна удовлетворять уравнению

$$
\begin{aligned}
\mathscr{H}_{2}^{\mathfrak{s} \mathfrak{l}_{\ell+1}} & \Psi_{\lambda_{1}, \ldots, \lambda_{\ell+1}}^{\mathfrak{s l}_{\ell+1}}\left(x_{1}, \ldots, x_{\ell+1}\right) \\
= & \left\{-\frac{1}{2} \sum_{i=1}^{\ell+1} \frac{\partial^{2}}{\partial x_{i}^{2}}+\sum_{i=1}^{\ell} e^{x_{i+1}-x_{i}}\right\} \Psi_{\lambda_{1}, \ldots, \lambda_{\ell+1}}^{\mathfrak{s} \mathfrak{l}_{\ell+1}}\left(x_{1}, \ldots, x_{\ell+1}\right) \\
= & \frac{1}{2} \sum_{i=1}^{\ell+1} \lambda_{i}^{2} \Psi_{\lambda_{1}, \ldots, \lambda_{\ell+1}}^{\mathfrak{s l} \ell+1}\left(x_{1}, \ldots, x_{\ell+1}\right)
\end{aligned}
$$


с дополнительным ограничением $\lambda_{1}+\cdots+\lambda_{\ell+1}=0$. Собственные функции $\mathfrak{s l}_{\ell+1}$-цепочки Тоды также можно записать, используя следующий набор переменных:

$\Psi_{\nu_{1}, \ldots, \nu_{\ell}}^{\mathfrak{s l}_{\ell+1}}\left(y_{1}, \ldots, y_{\ell}\right) \equiv \Psi_{\lambda_{1}, \ldots, \lambda_{\ell+1}}^{\mathfrak{s l}_{\ell+1}}\left(x_{1}, \ldots, x_{\ell+1}\right), \quad \nu_{i}=\lambda_{i+1}-\lambda_{i}, \quad y_{i}=x_{i+1}-x_{i}$.

Заметим, что если не налагать ограничение $\lambda_{1}+\cdots+\lambda_{\ell+1}=0$, то собственные функции $\mathfrak{s l}_{\ell+1}$-цепочки Тоды можно выразить через собственные функции $\mathfrak{g l}_{\ell+1}$-цепочки Тоды следующим образом:

$$
\Psi_{\nu_{1}, \ldots, \nu_{\ell}}^{\mathfrak{s} \mathfrak{l}_{\ell+1}}\left(y_{1}, \ldots, y_{\ell}\right)=\exp \left\{-\frac{\imath}{\ell+1} \sum_{i=1}^{\ell+1} \lambda_{i} \cdot \sum_{i=1}^{\ell+1} x_{i}\right\} \Psi_{\lambda_{1}, \ldots, \lambda_{\ell+1}}^{\mathfrak{g l}_{\ell+1}}\left(x_{1}, \ldots, x_{\ell+1}\right) .
$$

Здесь использованы обозначения из (1.30). Далее мы рассматриваем в основном собственные функции $\mathfrak{g l}_{\ell+1}$-цепочки Тоды, ограничиваясь комментариями относительно случая $\mathfrak{s l}_{\ell+1}$, и преимущественно используем неприведенную форму $\Psi_{\lambda}^{\mathfrak{g l}} \ell+1(x)$.

1.2.1. $\mathfrak{g l}_{\ell+1}$-функция Уиттекера: факторизационная параметризация. Для того чтобы сделать интегральное представление (1.19) для $\mathfrak{g l}_{\ell+1}$-функции Уиттекера явным, нам нужно выбрать специальную параметризацию $N_{+}^{(0)} \subset$ $\mathrm{GL}(\ell+1)$. Пусть $w_{0}$ - элемент максимальной длины в группе Вейля $S_{\ell+1}$ алгебры Ли $\mathfrak{g l}_{\ell+1}$. Рассмотрим приведенное разложение элемента $w_{0}$, отвечающее приведенному слову $I_{\ell}$ :

$$
I_{\ell}=\left(i_{1}, i_{2}, i_{3}, \ldots, i_{m_{\ell}}\right):=(1,21,321, \ldots,(\ell \ldots 21)), \quad m_{\ell}=\frac{\ell(\ell+1)}{2} .
$$

Заметим, что приведенное слово $I_{\ell}$ имеет очевидную рекурсивную структуру: $I_{\ell}=I_{\ell-1} \sqcup(\ell \ldots 21)$. Тем самым, соответствующая параметризация унипотентных элементов $v^{(\ell)}$ на открытом подмножестве $N_{+}^{(0)}$ подгруппы $N_{+}$может быть записана рекурсивно:

$$
v^{(\ell)}=v^{(\ell-1)} \mathfrak{X}_{\ell-1}^{\ell},
$$

где

$$
\mathfrak{X}_{\ell-1}^{\ell}=X_{\ell}\left(y_{\ell, 1}\right) \cdots X_{2}\left(y_{2, \ell-1}\right) X_{1}\left(y_{1, \ell}\right)
$$

и $X_{i}(y)=\exp \left(y f_{i}\right)$. Параметры $y_{i, k}$ однопараметрических подгрупп будем называть параметрами факторизации. Рассмотренное в п. 1.1.2 действие алгебры Ли $\mathfrak{g l}_{\ell+1}$ на $G / B_{-}$определяет действие алгебры Ли на пространстве функций $V_{\mu}$, ограниченном на $N_{+}^{(0)}$.

ПредлОженИЕ 1.2. Следующие дифференииальные операторы реализуют представление $\pi_{\mu}$ алгебры $\mathscr{U}\left(\mathfrak{g l}_{\ell+1}\right)$ в пространстве $V_{\mu}$ в терминах факторизационной параметризации (1.32), (1.33):

$$
E_{i, i+1}=\frac{\partial}{\partial y_{i, \ell+1-i}}+\sum_{k=1}^{i} \sum_{s=0}^{k-1} \frac{y_{i-1-s, \ell+2-i}}{y_{i-s, \ell+1-i}}\left\{\frac{\partial}{\partial y_{i-1-k, \ell+1-i}}-\frac{\partial}{\partial y_{i-1-k, \ell+2-i}}\right\},
$$




$$
\begin{aligned}
E_{i, i} & =\mu_{i}-\sum_{k=1}^{\ell+1-i} y_{i, k} \frac{\partial}{\partial y_{i, k}}+\sum_{k=1}^{\ell+2-i} y_{i-1, k} \frac{\partial}{\partial y_{i-1, k}}, \\
E_{i+1, i} & =\sum_{n=1}^{\ell+1-i} y_{i, n}\left\{\mu_{i}-\mu_{i+1}-y_{i, n} \frac{\partial}{\partial y_{i, n}}-\sum_{j=1}^{\ell} a_{i j} \sum_{k=1}^{n-1+\delta_{i, j+1}} y_{j, k} \frac{\partial}{\partial y_{j, k}}\right\},
\end{aligned}
$$

где $E_{i, j}=\pi_{\mu}\left(e_{i, j}\right), \mu_{k}=\imath \lambda_{k}-\rho_{k} u \rho_{k}=-(\ell-2 k+2) / 2$ nрu $k=1, \ldots, \ell+1$.

Доказательство приведено в п. 2.4.1 части 2.

Вычисление матричных элементов, входящих в интеграл (1.19), в факторизационной параметризации (1.32), (1.33) можно провести следуя [16] и [15] (подробности даны в разделе 2.3). Другой, более прямой подход, состоит в нахождении левого и правого векторов Уиттекера посредством решения уравнений (1.7), (1.8). Всюду далее мы считаем, что $\sum_{i=k}^{j}=0$ при $k>j$ и $\prod_{i=k}^{j}=1$ при $k>j$.

Напомним, что в соответствии с $(1.7),(1.8)$ векторы Уиттекера являются решениями уравнений

$$
E_{i, i+1} \psi_{R}=-\psi_{R}, \quad E_{i+1, i} \psi_{L}=-\psi_{L}, \quad i=1, \ldots, \ell .
$$

Лемма 1.3. Имеют место следующие выражения для левого/правого векторов Уиттекера в терминах параметров факторизаиии:

$$
\begin{aligned}
& \psi_{R}(y)= \exp \left\{-\sum_{i=1}^{\ell} \sum_{n=1}^{\ell+1-i} y_{i, n}\right\}, \\
& \psi_{L}(y)=\prod_{i=1}^{\ell}\left(\prod_{k=i}^{\ell} \prod_{n=1}^{i} y_{k+1-k, n}\right)^{\mu_{i+1}-\mu_{i}} \\
& \times \exp \left\{-\sum_{k=1}^{\ell} \frac{1}{y_{\ell+1-k, k}}\left(1+\sum_{n=1}^{\ell-k} \prod_{i=1}^{n} \frac{y_{\ell+1-k-i, k+1}}{y_{\ell+1-k-i, k}}\right)\right\} .
\end{aligned}
$$

С учетом (1.21) мы получаем следующее выражение $\mathfrak{g l}_{\ell+1}$-функции Уиттекера в факторизационной параметризации.

Теорема 1.1. Собственные функиии $\mathfrak{g l}_{\ell+1}$-цепочки Тоды (1.27) допускают интегральное представление:

$$
\begin{aligned}
\Psi_{\lambda_{1}, \ldots, \lambda_{\ell+1}}^{\mathfrak{g l} \mathfrak{l}_{\ell+1}} & \left(x_{1}, \ldots, x_{\ell+1}\right) \\
= & e^{\ell \sum_{k=1}^{\ell+1} \lambda_{k} x_{k}} \int_{C} \bigwedge_{i=1}^{\ell} \bigwedge_{n=1}^{\ell+1-i} \frac{d y_{i, n}}{y_{i, n}} \prod_{i=1}^{\ell}\left(\prod_{k=i}^{\ell} \prod_{n=1}^{i} y_{k+1-n, n}\right)^{\imath\left(\lambda_{i+1}-\lambda_{i}\right)} \\
& \times \exp \left\{-\sum_{k=1}^{\ell} \frac{1}{y_{\ell+1-k, k}}\left(1+\sum_{n=1}^{\ell-k} \prod_{i=1}^{n} \frac{y_{\ell+1-k-i, k+1}}{y_{\ell+1-k-i, k}}\right)\right. \\
& \left.-\sum_{i=1}^{\ell} e^{x_{i+1}-x_{i}} \sum_{n=1}^{\ell+1-i} y_{i, n}\right\}
\end{aligned}
$$


Здесъ $C \subset N_{+}-$некомпактное подмногообразие половинной размерности, для которого подынтегральные функиии экспоненииально убывают на границе и на бесконечности. $B$ частности, в качестве $C$ можно взять малую дебормацию подмножества $\mathbb{R}_{+}^{\ell(\ell+1) / 2}{ }_{\text {в }} \mathbb{C}^{\ell(\ell+1) / 2}$, для которой интеграл $(1.37)$ сходится.

Доказательство дано в п. 2.3.1 части 2.

1.2.2. $\mathfrak{g l}_{\ell+1}$-функция Уиттекера: модифицированная факторизационная параметризация. Рассмотрим модификацию факторизационной параметризации (1.32), (1.33), которая приводит к интегральному представлению Гивенталя $\mathfrak{g l}_{\ell+1}$-функции Уиттекера. Эта модифицированная параметризация впервые возникла в работе [6]. Следует подчеркнуть, что между факторизационной и модифицированной факторизационной параметризациями имеется принципиальное отличие. А именно, параметризация (1.32), (1.33) определяется в терминах элементов подгруппы $N_{+}$. В свою очередь, для того чтобы определить модифицированную факторизационную параметризацию на $N_{+}^{(0)}$, мы рассматриваем образ элемента группы в точном конечномерном представлении данной группы. В случае алгебр $\mathfrak{g l}_{\ell+1}$ и $\mathfrak{s l}_{\ell+1}$ мы используем тавтологическое представление $\pi: \mathfrak{g l}_{\ell+1} \rightarrow \operatorname{End}\left(\mathbb{C}^{\ell+1}\right)$. Пусть $\epsilon_{i, j}-$ множество элементарных $(\ell+1) \times(\ell+1)$-матриц с единицей на месте $(i, j)$ и с нулями на остальных местах. Рассмотрим диагональные матрицы

$$
U_{k}=\sum_{i=1}^{k} e^{-x_{k, i}} \epsilon_{i, i}+\sum_{i=k+1}^{\ell+1} \epsilon_{i, i}
$$

и их верхнетреугольные деформации:

$$
\widetilde{U}_{k}=\sum_{i=1}^{k} e^{-x_{k, i}} \epsilon_{i, i}+\sum_{i=k+1}^{\ell+1} \epsilon_{i, i}+\sum_{i=1}^{k-1} e^{-x_{k-1, i}} \epsilon_{i, i+1} .
$$

Модифицированная факторизационная параметризация на $N_{+}$определяется следующим образом.

Теорема 1.2. (i) Образ типичного унипотентного элемента $v \in N_{+} 8$ тавтологическом представлении $\pi: \mathfrak{g l}_{\ell+1} \rightarrow \operatorname{End}\left(\mathbb{C}_{\ell+1}\right)$ можно представить в виде

$$
\pi(v)=\widetilde{U}_{2} U_{2}^{-1} \widetilde{U}_{3} U_{3}^{-1} \cdots \widetilde{U}_{\ell} U_{\ell}^{-1} \widetilde{U}_{\ell+1},
$$

где предполагается, что $x_{\ell+1, i}=0, i=1, \ldots, \ell+1$.

(ii) Формула (1.40) определяет параметризацию множества полностью неотрицательных унипотентных элементов $N_{+}^{(0)} \subset N_{+}$.

ДокАЗАТЕЛЬСтво. Пусть $v(y)$ - элементы из $N_{+}$, параметризованные в соответствии с (1.32), (1.33). Произведем следующую замену переменных:

$$
y_{i, n}=e^{x_{n+i, i+1}-x_{n+i-1, i}}, \quad 1 \leqslant n \leqslant i \leqslant \ell,
$$

где $x_{\ell+1, n}=0, n=1, \ldots, \ell+1$. Несложно проверить, что после такой замены образ $\pi(v)$ элемента $v$, заданного в $(1.32),(1.33)$, преобразуется в (1.40). Учитывая, что замена переменных (1.41) обратима, мы получаем параметризацию на подмножестве $N_{+}^{(0)} \subset N_{+}$. Теорема 1.2 доказана. 
Применяя замену переменных (1.41) к выражениям из предложения 1.2, мы получаем искомую реализацию в модифицированной факторизационной параметризации.

ПредложениЕ 1.3. Следующие дифференииалъные операторы определяют реализацию представления $\pi_{\mu}$ алгебры $\mathfrak{g l}_{\ell+1}$ в пространстве $V_{\mu}$ в терминах модифицированной факторизационной параметризащии (1.38)-(1.40) на групne $N_{+}$:

$$
\begin{aligned}
E_{i, i} & =\mu_{i}-\sum_{k=1}^{i-1} \frac{\partial}{\partial x_{\ell+1+k-i, k}}+\sum_{k=i}^{\ell} \frac{\partial}{\partial x_{k, i}}, \\
E_{i, i+1} & =-\sum_{n=1}^{i} e^{x_{\ell-i+n, n}-x_{\ell+1-i+n, n}} \sum_{k=1}^{n}\left\{\frac{\partial}{\partial x_{\ell-i+k, k}}-\frac{\partial}{\partial x_{\ell-i+k, k-1}}\right\}, \\
E_{i+1, i} & =\sum_{n=1}^{\ell+1-i} e^{x_{n+i, i+1}-x_{k+i-1, i}}\left[\mu_{i}-\mu_{i+1}+\sum_{k=1}^{n}\left\{\frac{\partial}{\partial x_{i+k-1, i}}-\frac{\partial}{\partial x_{i+k-1, i+1}}\right\}\right],
\end{aligned}
$$

где $E_{i, j}=\pi_{\mu}\left(e_{i, j}\right)$ u $x_{\ell+1, i}=0$ при $i=1, \ldots, \ell+1$.

Реализация дифференциальными операторами представления основной серии алгебры $\mathfrak{g l}_{\ell+1}$ основывается на специальной параметризации максимальной унипотентной подгруппы $N_{+}$, входящей в разложение Гаусса группы GL $(\ell+1)$. Она возникла благодаря интегральной формуле Гивенталя. В работе [6] мы предложили использовать для этой реализации представления основной серии термин "представление Гаусса-Гивенталя". Используя замену переменных (1.41) в выражениях из леммы 1.3, мы получаем векторы Уиттекера в модифицированной факторизационной параметризации.

ЛЕмма 1.4. Имеют место следующие выражения для левого и правого векторов Уиттекера:

$$
\begin{aligned}
& \psi_{R}(x)=\exp \left\{-\sum_{i=1}^{\ell} \sum_{n=1}^{\ell+1-i} e^{x_{n+i, i+1}-x_{n+i-1, i}}\right\} \\
& \psi_{L}(x)=\exp \left\{\sum_{k=1}^{\ell} \sum_{i=1}^{k}\left(\mu_{k}-\mu_{k+1}\right) x_{k, i}\right\} \exp \left\{-\sum_{i=1}^{\ell} \sum_{k=1}^{\ell+1-i} e^{x_{k+i-1, k}-x_{k+i, k}}\right\},
\end{aligned}
$$

где $x_{\ell+1, i}=0, i=1, \ldots, \ell+1$.

Теперь мы готовы предъявить интегральное представление для спаривания (1.27) с использованием модифицированной факторизационной параметризации. Переходя от (1.27) к (1.19), а затем к (1.37), мы действуем элементом подгруппы Картана $e^{\sum x_{i} E_{i, i}}$ на (1.27) справа. Другой выбор действия (например, левого действия) приводит к преобразованию подынтегрального выражения добавлением к нему полного дифференциала от некоторой функции. Выбор диагонального элемента в (1.19), (1.37) не является наиболее симметричным. Одно из специальных свойств представления Гаусса-Гивенталя состоит 
в том, что с точностью до простого экспоненциального множителя в $\psi_{L}(x)$ левый и правый векторы Уиттекера (1.43) очень похожи (это не так в случае факторизационной параметризации (1.17), (1.18)). Нам бы хотелось сохранить эту симметрию в подынтегральном выражении интегрального представления. Представим элемент подгруппы Картана $H$ в виде

$$
e^{H}=e^{H_{L}} e^{H_{R}},
$$

где

$$
\begin{aligned}
e^{H} & =e^{\sum x_{i} E_{i, i}}=\exp \left\{\sum_{i=1}^{\ell} x_{\ell+1, i}\left(\mu_{i}-\sum_{k=1}^{i-1} \frac{\partial}{\partial x_{\ell+1+k-i, k}}+\sum_{k=i}^{\ell} \frac{\partial}{\partial x_{k, i}}\right)\right\}, \\
e^{H_{L}} & =\exp \left\{\sum_{i=1}^{\ell+1} x_{\ell+1, i} \sum_{k=i}^{\ell} \frac{\partial}{\partial x_{k, i}}\right\}, \\
e^{H_{R}} & =\exp \left\{\sum_{i=1}^{\ell+1} x_{\ell+1, i} \mu_{i}-\sum_{i=1}^{\ell+1} x_{\ell+1, i} \sum_{k=1}^{i-1} \frac{\partial}{\partial x_{\ell+1+k-i, k}}\right\} .
\end{aligned}
$$

При вычислении матричного элемента мы будем выбирать дифференциальный оператор $H_{L}$, действующий на левом векторе, и оператор $H_{R}$, действующий на правом векторе Уиттекера в (1.27). Так мы получим следующую интегральную формулу для собственных функций $\mathfrak{g l}_{\ell+1}$-цепочки Тоды.

ТЕорема 1.3. Собственные функиии $\mathfrak{g l}_{\ell+1}$-цепочки Тоды (1.27) допускают следующее интегральное представление:

$$
\Psi_{\lambda_{1}, \ldots, \lambda_{\ell+1}}^{\mathfrak{g l}_{\ell+1}}\left(x_{1}, \ldots, x_{\ell+1}\right)=\int_{C} \prod_{k=1}^{\ell} d \underline{x}_{k} e^{\mathscr{F}^{\mathfrak{g} \mathfrak{l}_{\ell+1}(x)}},
$$

где $\underline{x}_{k}=\left(x_{k, 1}, \ldots, x_{k, k}\right), k=1, \ldots, \ell$, а функция $\mathscr{F}^{\mathfrak{g l}_{\ell+1}}(x)$ задана как $\mathscr{F}^{\mathfrak{g l}_{\ell+1}}(x)=\imath \sum_{n=1}^{\ell+1} \lambda_{n}\left(\sum_{i=1}^{n} x_{n, i}-\sum_{i=1}^{n-1} x_{n-1, i}\right)-\sum_{k=1}^{\ell} \sum_{i=1}^{k}\left(e^{x_{k, i}-x_{k+1, i}}+e^{x_{k+1, i+1}-x_{k, i}}\right)$.

Здесь $x_{i}=x_{\ell+1, i}, i=1, \ldots, \ell+1, a C \subset N_{+}$- некомпактное подмногообразие половинной размерности, для которого подынтегральные функции экспоненииально убывают на границе и на бесконечности. Например, С можно получить малой дебормацией подпространства $\mathbb{R}^{(\ell+1) \ell / 2} \subset \mathbb{C}^{(\ell+1) \ell / 2}$, обеспечивающей сходимость интеграла (1.47).

Отметим, что указанное выше интегральное представление (1.47), (1.48) можно получить непосредственно из (1.37), подставляя

$$
y_{i, n}=e^{x_{\ell+1, i}-x_{\ell+1, i+1}} e^{x_{n+i, i+1}-x_{n+i-1, i}}, \quad i=1, \ldots, \ell, \quad n=1, \ldots, \ell+1-i .
$$

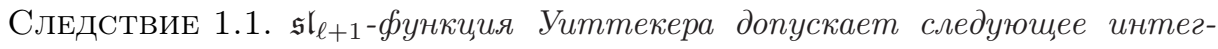
ральное представление:

$$
\begin{aligned}
& \Psi_{\lambda_{2}-\lambda_{1}, \ldots, \lambda_{\ell+1}-\lambda_{\ell}}^{\mathfrak{s l}_{\ell+1}}\left(x_{2}-x_{1}, \ldots, x_{\ell+1}-x_{\ell}\right) \\
& \quad=\exp \left\{\frac{\imath}{\ell+1} \sum_{i=1}^{\ell+1} x_{i}\left(\ell \lambda_{i}-\sum_{j \neq i} \lambda_{j}\right)\right\} \int_{C} \prod_{k=1}^{\ell} d \underline{x}_{k} e^{\mathscr{F}^{\mathfrak{s} \boldsymbol{l} \ell+1}(x)},
\end{aligned}
$$


¿əe $\mathscr{F}^{\mathfrak{s l}_{\ell+1}}(x)=\imath \sum_{k=1}^{\ell}\left(\lambda_{k+1}-\lambda_{k}\right) \sum_{i=1}^{k}\left(x_{\ell+1, k}-x_{k, i}\right)-\sum_{k=1}^{\ell} \sum_{i=1}^{k}\left(e^{x_{k, i}-x_{k+1, i}}+e^{x_{k+1, i+1}-x_{k, i}}\right)$ $u x_{k}=x_{\ell+1, k}, k=1, \ldots, \ell+1$.

ПримеР 1.1. Пусть $x_{1}=x_{2,1}, x_{2}=x_{2,2}$. В случае $\ell=1$ представление (1.47), (1.48) превращается в

$$
\Psi_{\lambda_{1}, \lambda_{2}}^{\mathfrak{g l} l_{2}}\left(x_{1}, x_{2}\right)=\int_{\mathbb{R}} d x_{1,1} \exp \left\{\imath \lambda_{2}\left(x_{1}+x_{2}-x_{1,1}\right)+\imath \lambda_{1} x_{1,1}-e^{x_{1,1}-x_{1}}-e^{x_{2}-x_{1,1}}\right\} .
$$

Здесь $\mathfrak{s l}_{2}$-функция Уиттекера (1.50) совпадает с так называемой модифицированной функцией Бесселя 2-го рода [9]:

$$
\begin{aligned}
\Psi_{\lambda_{2}-\lambda_{1}}^{\mathfrak{s l}_{2}}\left(x_{2}-x_{1}\right) & =e^{\frac{\imath}{2}\left(\lambda_{2}-\lambda_{1}\right)\left(x_{1}+x_{2}\right)} \int_{\mathbb{R}} d x_{1,1} e^{\imath\left(\lambda_{1}-\lambda_{2}\right) x_{1,1}-e^{x_{1,1}-x_{1}}-e^{x_{2}-x_{1,1}}} \\
& =\int_{\mathbb{R}} d x_{1,1} \exp \left\{\imath\left(\lambda_{1}-\lambda_{2}\right) x_{1,1}-e^{\frac{x_{2}-x_{1}}{2}}\left(e^{x_{1,1}}+e^{-x_{1,1}}\right)\right\} \\
& =K_{\imath\left(\lambda_{1}-\lambda_{2}\right)}\left(2 e^{\frac{x_{2}-x_{1}}{2}}\right) .
\end{aligned}
$$

Интегральное представление (1.47), (1.48) для собственных функций $\mathfrak{g l}_{\ell+1^{-}}$ цепочки Тоды было впервые получено Гивенталем в его работе о квантовых когомологиях $\mathfrak{g l}_{\ell+1}$-многообразия флагов [1] (см. также [2]). Теоретико-представленческий вывод интегральной формулы Гивенталя в виде матричного элемента (1.27) впервые появился в работе [6].

Согласно Гивенталю, функция $\mathscr{F}^{\mathfrak{g l}_{\ell+1}}(x)$ при $\lambda_{k}=0, k=1, \ldots, \ell+1$, допускает простое описание в терминах следующей диаграммы:

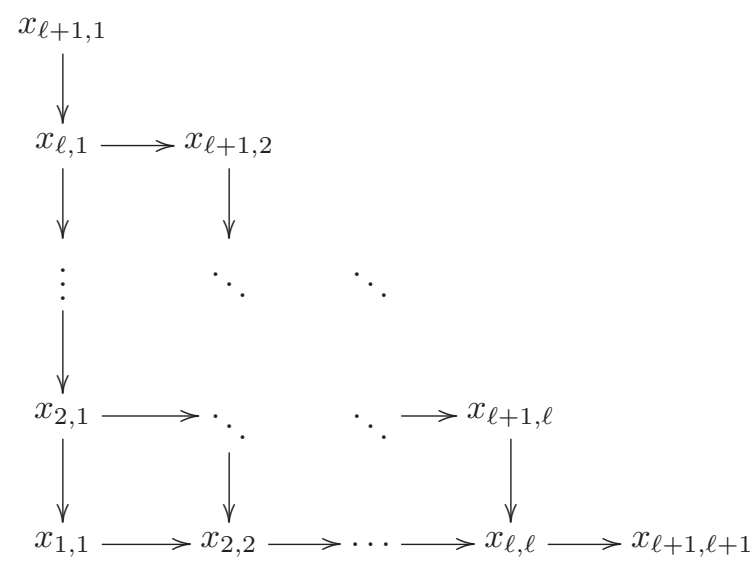


Мы связываем переменные $x_{k, i}$ с вершинами $(k, i)$ и функции $e^{y-x}$ со стрелками $(x \longrightarrow y)$ диаграммы $(1.51)$. Тогда потенциал $\mathscr{F}^{\mathfrak{g l}_{\ell+1}}(x)$ из $(1.48)$ на нулевом уровне спектра $\lambda_{i}=0$ задается суммой функций, отвечающих всем стрелкам.

Как показано в теореме 1.2 , переменные $\left\{x_{k, i}, 1 \leqslant i \leqslant k \leqslant \ell\right\}$ определяют параметризацию открытого подмножества $N_{+}^{(0)}$ многообразия флагов $X=$ $\mathrm{SL}(\ell+1, \mathbb{C}) / B$. Некомпактное многообразие $N_{+}^{(0)}$ обладает естественным действием тора $T^{m_{\ell}}$ и допускает компактификацию, которая является горенштейновым торическим многообразием. Множество соотношений между мономами, определяющее эту компактификацию, можно описать следующим образом. Введем новые переменные

$$
a_{k, i}=e^{x_{k, i}-x_{k+1, i}}, \quad b_{k, i}=e^{x_{k+1, i+1}-x_{k, i}}, \quad 1 \leqslant i \leqslant k \leqslant \ell
$$

связанные со стрелками диаграммы (1.51). Тогда имеют место соотношения

$$
\begin{gathered}
a_{k, i} b_{k, i}=b_{k+1, i} a_{k+1, i+1}, \quad 1 \leqslant i \leqslant k<\ell, \\
a_{\ell, i} b_{\ell, i}=e^{x_{\ell+1, i+1}-x_{\ell+1, i}}, \quad i=1, \ldots, \ell .
\end{gathered}
$$

Их можно интерпретировать как соотношения между различными композициями элементарных путей с совпадающими исходной и конечной вершинами. Множество соотношений между более общими путями (следующих из (1.52)) определяет торическое вложение вырождения многообразия флагов (подробности см. в [5] и [19]). Отметим, что диаграмма (1.51) совпадает с диаграммой Гельфанда-Цейтлина для алгебры Ли $\mathfrak{g l}_{\ell+1}$.

1.2.3. Связь с Q2-оператором Бакстера $\widehat{\mathfrak{g l}}_{\ell+1}$-цепочки Тоды. Интегральное представление (1.47), (1.48) для $\mathfrak{g l}_{\ell+1}$-функции Уиттекера имеет рекурсивную структуру относительно ранга $\ell+1$ алгебры Ли. В самом деле, это интегральное представление можно переписать в следующем виде:

$$
\Psi_{\lambda_{1}, \ldots, \lambda_{\ell+1}}^{\mathfrak{g l}_{\ell+1}}\left(\underline{x}_{\ell+1}\right)=\int_{C} \prod_{k=1}^{\ell} d \underline{x}_{k} \prod_{k=1}^{\ell+1} Q_{\mathfrak{g l}_{k-1}}^{\mathfrak{g l}_{k}}\left(\underline{x}_{k}, \underline{x}_{k-1} ; \lambda_{k}\right),
$$

где

$$
\begin{aligned}
Q_{\mathfrak{g l}_{k}}^{\mathfrak{g l}_{k+1}}\left(\underline{x}_{k+1}, \underline{x}_{k} ; \lambda_{k+1}\right)=\exp \{ & \imath \lambda_{k+1}\left(\sum_{i=1}^{k+1} x_{k+1, i}-\sum_{i=1}^{k} x_{k, i}\right) \\
& \left.-\sum_{i=1}^{k}\left(e^{x_{k, i}-x_{k+1, i}}+e^{x_{k+1, i+1}-x_{k, i}}\right)\right\} .
\end{aligned}
$$

Здесь использовано обозначение $\underline{x}_{k}=\left(x_{k, 1}, \ldots, x_{k, k}\right)$ и предполагается, что $Q_{\mathfrak{g l}_{0}}^{\mathfrak{g l}_{1}}=e^{\imath \lambda_{1} x_{1,1}}$.

Выберем линейные координаты $\underline{x}_{k}=\left(x_{k, 1}, \ldots, x_{k, k}\right)$ в пространстве $\mathbb{C}^{k}$. Пусть $\mathscr{C}_{k}-$ некомпактное подмногообразие половинной размерности в $\mathbb{C}^{k}$, для которого (1.54) как функция от $\underline{x}_{k}$ экспоненциально убывает на возможных 
границах и на бесконечности $\mathscr{C}_{k}$. Рассмотрим следующий интегральный оператор:

$$
\left(Q_{\mathfrak{g l}_{k}}^{\mathfrak{g l}_{k+1}} f\right)\left(\underline{x}_{k+1}\right)=\int_{\mathscr{C}_{k}} Q_{\mathfrak{g l}_{k}}^{\mathfrak{g l}_{k+1}}\left(\underline{x}_{k+1}, \underline{x}_{k} ; \lambda_{k+1}\right) f\left(\underline{x}_{k}\right) d \underline{x}_{k}
$$

Он действует на функциях, которые растут не слишком быстро на возможных границах и на бесконечности в $\mathscr{C}_{k}$. Интегральные операторы $Q_{\mathfrak{g l}}^{\mathfrak{g l}_{k+1}}$ определяют рекурсивную конструкцию $\mathfrak{g l}_{\ell+1}$-функций Уиттекера:

$$
\Psi_{\lambda_{1}, \ldots, \lambda_{\ell+1}}^{\mathfrak{g l} l_{\ell+1}}\left(\underline{x}_{\ell+1}\right)=\int_{\mathscr{C}_{\ell}} d \underline{x}_{\ell} Q_{\mathfrak{g l}_{\ell}}^{\mathfrak{g l} l_{\ell+1}}\left(\underline{x}_{\ell+1}, \underline{x}_{\ell} ; \lambda_{\ell+1}\right) \Psi_{\lambda_{1}, \ldots, \lambda_{\ell}}^{\mathfrak{g l} \mathfrak{l}_{\ell}}\left(\underline{x}_{\ell}\right) .
$$

С оператором рекурсии $Q_{\mathfrak{g l}_{\ell}}^{\mathfrak{g l}_{\ell+1}}$ можно связать ориентированный путь на графe (1.51):

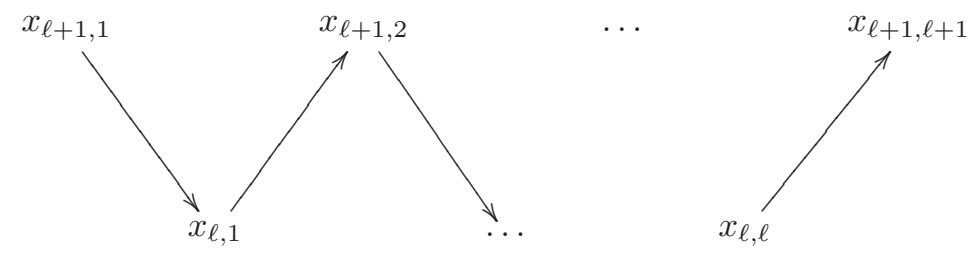

Диаграмму (1.51) можно рассматривать как набор ориентированных путей (1.56), и тем самым рекурсивная структура интегрального представления (1.47) закодирована в диаграмме (1.51) очевидным образом.

В силу (1.55) интегральные операторы $Q_{\mathfrak{g l}_{k}}^{\mathfrak{g l}_{k+1}}$ с ядрами $Q_{\mathfrak{g l}_{k+1}}^{\mathfrak{g l}_{k+1}}\left(\underline{x}_{k+1}, \underline{x}_{k} ; \lambda_{k+1}\right)$ удовлетворяют сплетающим соотношениям для гамильтонианов квантовых цепочек Тоды. Например, между квадратичными гамильтонианами $\mathscr{H}_{2}^{\mathfrak{g l}_{k+1}}\left(\underline{x}_{k+1}\right)$ и $\mathscr{H}_{2}^{\mathfrak{g l}_{k}}\left(\underline{x}_{k}\right)$ имеет место соотношение

$$
\mathscr{H}_{2}^{\mathfrak{g l}_{k+1}}\left(\underline{x}_{k+1}\right) Q_{\mathfrak{g l}_{k}}^{\mathfrak{g l}_{k+1}}\left(\underline{x}_{k+1}, \underline{x}_{k} ; \lambda_{k+1}\right)=Q_{\mathfrak{g l}_{k}}^{\mathfrak{g l}_{k+1}}\left(\underline{x}_{k+1}, \underline{x}_{k} ; \lambda_{k+1}\right) \mathscr{H}_{2}^{\mathfrak{g l}_{k}}\left(\underline{x}_{k}\right)+\frac{1}{2} \lambda_{k+1}^{2} .
$$

Мы будем предполагать, что в подобных соотношениях гамильтоновы операторы в левой части действуют справа, а гамильтоновы операторы в правой части действуют слева. Похожие коммутационные соотношения имеют место для высших квантовых гамильтоновых операторов (подробности см. в [6]).

Операторы рекурсии $Q_{\mathfrak{g l}_{k+1}}^{\mathfrak{g l}_{k+1}}$ тесно связаны с важным объектом теории квантовых интегрируемых систем, который называется $\mathscr{Q}$-оператором. Специальный вид Q-оператора был введен Р. Бакстером в работе [31] для решения одного класса квантовых интегрируемых моделей. В случае (периодической) $\widehat{\mathfrak{g l}}_{\ell+1}$-цепочки Тоды с квадратичным гамильтонианом

$$
\mathscr{H}_{2}^{\widehat{\mathfrak{g l}}_{\ell+1}}=-\frac{1}{2} \sum_{i=1}^{\ell+1} \frac{\partial^{2}}{\partial x_{i}^{2}}+\sum_{i=1}^{\ell} e^{x_{i+1}-x_{i}}+g e^{x_{1}-x_{\ell+1}},
$$


где $g$ - произвольная константа связи, Q-оператор имеет следующее интегральное ядро:

$$
\begin{aligned}
\mathscr{Q}^{\widehat{\mathfrak{g}}_{\ell+1}}\left(\underline{x}^{(\ell+1)}, \underline{y}^{(\ell+1)} ; \lambda\right) & \exp \left\{\imath \lambda \sum_{i=1}^{\ell+1}\left(x_{i}-y_{i}\right)\right. \\
& \left.-\left(\sum_{i=1}^{\ell}\left(e^{y_{i}-x_{i}}+e^{x_{i+1}-y_{i}}\right)+e^{y_{\ell+1}-x_{\ell+1}}+g e^{x_{1}-y_{\ell+1}}\right)\right\} .
\end{aligned}
$$

Здесь мы используем обозначения $\underline{x}^{(\ell+1)}=\left(x_{1}, \ldots, x_{\ell+1}\right)$ и $\underline{y}^{(\ell+1)}=\left(y_{1}, \ldots, y_{\ell+1}\right)$. Этот $\mathscr{Q}$-оператор был впервые построен в работе [10]. Он коммутирует со всеми гамильтонианами $\widehat{\mathfrak{g l}}_{\ell+1}$-цепочки Тоды и порождает квантовые преобразования Бэклунда [10]. Например, для квадратичных гамильтонианов мы получаем:

$$
\mathscr{H}_{2}^{\widehat{\mathfrak{g l}}_{\ell+1}}\left(\underline{x}^{(\ell+1)}\right) \mathscr{Q}^{\widehat{\mathfrak{g}}_{\ell+1}}\left(\underline{x}^{(\ell+1)}, \underline{y}^{(\ell+1)} ; \lambda\right)=\mathscr{Q}^{\widehat{\mathfrak{g}}_{\ell+1}}\left(\underline{x}^{(\ell+1)}, \underline{y}^{(\ell+1)} ; \lambda\right) \mathscr{H}_{2}^{\widehat{\mathfrak{g} l}_{\ell+1}}\left(\underline{y}^{(\ell+1)}\right) \text {. }
$$

Для установления связи между $\mathscr{Q}$-оператором Бакстера для $\widehat{\mathfrak{g l}}_{k+1}$-теории Тоды и оператором рекурсии для $\mathfrak{g l}_{k+1}$-теории Тоды полезно ввести несколько модифицированный оператор рекурсии $Q_{\mathfrak{g l}_{k} \oplus \mathfrak{g l}_{1}}^{\mathfrak{g l}_{k+1}}$ с ядром:

$$
\begin{array}{r}
Q_{\mathfrak{g l}_{k} \oplus \mathfrak{g l}_{1}}^{\mathfrak{g l}_{k+1}}\left(\underline{x}^{(k+1)}, \underline{y}^{(k+1)} ; \lambda\right)=e^{-\imath \lambda y_{k+1}} Q_{\mathfrak{g l}_{k}}^{\mathfrak{g l}_{k+1}}\left(\underline{x}^{(k+1)}, \underline{y}^{(k)} ; \lambda\right) \\
=\exp \left\{\imath \lambda\left(\sum_{i=1}^{k+1} x_{i}-\sum_{i=1}^{k+1} y_{i}\right)-\sum_{i=1}^{k}\left(e^{y_{i}-x_{i}}+e^{x_{i+1}-y_{i}}\right)\right\},
\end{array}
$$

где $\underline{x}^{(k+1)}=\left(x_{1}, \ldots, x_{k+1}\right), \underline{y}^{(k)}=\left(y_{1}, \ldots, y_{k}\right)$ и $\underline{y}^{(k+1)}=\left(y_{1}, \ldots, y_{k}, y_{k+1}\right)$.

Этот модифицированный оператор сплетает гамильтоновы операторы $\mathfrak{g l}_{k+1^{-}}$ и $\mathfrak{g l}_{k} \oplus \mathfrak{g l}_{1}$-цепочек Тоды (новая переменная $y_{k+1}$ входит только в описание $\mathfrak{g l}_{1}$-цепочки Тоды). Тем самым, для квадратичных гамильтоновых операторов имеем

$$
\begin{aligned}
\mathscr{H}_{2} \mathfrak{g l}_{k+1} & \left(\underline{x}^{(k+1)}\right) Q_{\mathfrak{g l}_{k} \oplus \mathfrak{g l}_{1}}^{\mathfrak{g l}_{k+1}}\left(\underline{x}^{(k+1)}, \underline{y}^{(k+1)} ; \lambda\right) \\
= & \left.Q_{\mathfrak{g l}_{k} \oplus \mathfrak{g l}_{1} \underline{\mathfrak{l}}_{k+1}}^{(k+1)}, \underline{y}^{(k+1)} ; \lambda\right)\left(\mathscr{H}_{2}^{\mathfrak{g l}_{k}}\left(\underline{y}^{(k)}\right)+\mathscr{H}_{2}^{\mathfrak{g l}^{(k+1}}\left(y_{k+1}\right)\right),
\end{aligned}
$$

где $\mathscr{H}_{2}^{\mathfrak{g l} l_{1}}\left(y_{k+1}\right)=-\frac{1}{2} \frac{\partial^{2}}{\partial y_{k+1}^{2}}$. Ясно, что проекция этого соотношения на подпространство функций $F\left(\underline{y}^{(k)}, y_{k+1}\right)=e^{-\imath \lambda y_{k+1}} f\left(\underline{y}^{(k)}\right)$ приводит к $(1.57)$.

Рассмотрим однопараметрическое семейство интегральных операторов

$$
\begin{aligned}
\mathscr{Q}^{\widehat{\mathfrak{g}}_{\ell+1}}\left(\underline{x}^{(k+1)}, \underline{y}^{(k+1)} ; \lambda ; \varepsilon\right)=\varepsilon^{-\imath \lambda} \exp \left\{\imath \lambda \sum_{i=1}^{\ell+1}\left(x_{i}-y_{i}\right)\right. \\
\left.-\left(\sum_{i=1}^{\ell}\left(e^{y_{i}-x_{i}}+e^{x_{i+1}-y_{i}}\right)+\varepsilon e^{y_{\ell+1}-x_{\ell+1}}+\varepsilon^{-1} g e^{x_{1}-y_{\ell+1}}\right)\right\},
\end{aligned}
$$

полученных из (1.59) сдвигом переменной $y_{\ell+1} \rightarrow y_{\ell+1}+\ln \varepsilon$. Предельное поведение операторов (1.60) при $\varepsilon \rightarrow 0, g \varepsilon^{-1} \rightarrow 0$ можно описать следующим 
образом:

$$
Q_{\mathfrak{g l}_{k+1} \mathfrak{g l}_{k} \oplus \mathfrak{g l}_{1}}\left(\underline{x}^{(k+1)}, \underline{y}^{(k+1)} ; \lambda\right)=\lim _{\substack{\varepsilon \rightarrow 0, g \varepsilon^{-1} \rightarrow 0}} \varepsilon^{\imath \lambda} \mathscr{Q}^{\widehat{\mathfrak{g l}}_{k+1}}\left(\underline{x}^{(k+1)}, \underline{y}^{(k+1)} ; \lambda ; \varepsilon\right) .
$$

Это устанавливает взаимосвязь между Q्Q-оператором Бакстера и (модифицированным) оператором рекурсии.

1.3. Интегральные представления собственных функций $\mathfrak{s o}_{2 \ell+1}$-цепочки Тоды. В этом разделе мы получим обобщение интегрального представления Гивенталя для $\mathfrak{g l}_{\ell+1}$-функций Уиттекера на случай алгебры Ли $\mathfrak{s o}_{2 \ell+1}$. Мы начнем с задания интегрального представления $\mathfrak{s o}_{2 \ell+1}$-функций Уиттекера, использующего факторизационное представление. Затем будет рассмотрена модификация факторизационного представления, которая приводит к интегральному представлению типа Гивенталя.

Рассмотрим систему корней типа $B_{\ell}$, отвечающую алгебре Ли $\mathfrak{s o}_{2 \ell+1}$. Пусть $\left\{\epsilon_{1}, \ldots, \epsilon_{\ell}\right\}$ - ортонормированный базис в пространстве $\mathbb{R}^{\ell}$. Реализуем корни, кокорни и фундаментальные веса, связанные с системой $B_{\ell}$, как векторы в $\mathbb{R}^{\ell}$ :

$$
\begin{array}{lll}
\alpha_{1}=\epsilon_{1}, & \alpha_{1}^{\vee}=2 \epsilon_{1}, & \omega_{1}=\frac{1}{2}\left(\epsilon_{1}+\cdots+\epsilon_{\ell}\right), \\
\alpha_{2}=\epsilon_{2}-\epsilon_{1}, & \alpha_{2}^{\vee}=\epsilon_{2}-\epsilon_{1}, & \omega_{2}=\epsilon_{2}+\cdots+\epsilon_{\ell}, \\
\ldots & \ldots & \cdots \\
\alpha_{\ell}=\epsilon_{\ell}-\epsilon_{\ell-1}, & \alpha_{\ell}^{\vee}=\epsilon_{\ell}-\epsilon_{\ell-1}, & \omega_{\ell}=\epsilon_{\ell} .
\end{array}
$$

Матрица Картана в этом случае имеет вид $a_{i j}=\left\langle\alpha_{i}^{\vee}, \alpha_{j}\right\rangle$. Для положительных рациональных чисел $d_{1}=1 / 2, d_{2}=1, \ldots, d_{\ell}=1$ матрица $\left\|b_{i j}\right\|=\left\|d_{i} a_{i j}\right\|$ симметрична. С этими данными связана квантовая цепочка Тоды с квадратичным гамильтонианом

$$
\mathscr{H}_{2}^{B_{\ell}}=-\frac{1}{2} \sum_{i=1}^{\ell} \frac{\partial^{2}}{\partial x_{i}^{2}}+\frac{1}{2} e^{x_{1}}+\sum_{i=1}^{\ell-1} e^{x_{i+1}-x_{i}}
$$

Можно дополнить (1.65) до полного набора из $\ell$ попарно коммутирующих функционально независимых гамильтонианов $\mathscr{H}_{k}^{B_{\ell}}, k=1, \ldots, \ell$, для $\mathfrak{s o}_{2 \ell+1}$-цепочки Тоды. Мы ищем интегральное представление общей собственной функции для $\mathscr{H}_{k}^{B_{\ell}}$. Задача о собственной функции квадратичного гамильтониана может быть записана в следующем виде:

$$
\mathscr{H}_{2}^{B_{\ell}} \Psi_{\lambda_{1}, \ldots, \lambda_{\ell}}^{B_{\ell}}\left(x_{1}, \ldots, x_{\ell}\right)=\frac{1}{2} \sum_{i=1}^{\ell} \lambda_{i}^{2} \Psi_{\lambda_{1}, \ldots, \lambda_{\ell}}^{B_{\ell}}\left(x_{1}, \ldots, x_{\ell}\right) .
$$

1.3.1. $\mathfrak{s o}_{2 \ell+1}$-функция Уиттекера: факторизационная параметризация. Приведенное слово, отвечающее элементу $w_{0}$ максимальной длины в группе Вейля типа $B_{\ell}$, можно представить рекурсивно:

$$
I_{\ell}=\left(i_{1}, i_{2}, i_{3}, \ldots, i_{m_{\ell}}\right):=(1,212,32123, \ldots,(\ell \ldots 212 \ldots \ell)), \quad m_{\ell}=\ell^{2},
$$


где индексы $i_{k} \in \Gamma=\{1, \ldots, \ell\}$ соответствуют отражениям относительно простых корней $\alpha_{i_{k}}$. Пусть $N_{+} \subset G$ - максимальная унипотентная подгруппа в $G=\mathrm{SO}(2 \ell+1)$. Свяжем с приведенным словом $I_{\ell}$ следующую рекурсивную параметризацию типичного унипотентного элемента $v^{B_{\ell}} \in N_{+}$:

$$
v^{B_{\ell}}=v^{B_{\ell-1}} \mathfrak{X}_{B_{\ell-1} B_{\ell}},
$$

где

$$
\begin{aligned}
\mathfrak{X}_{B_{\ell-1}}^{B_{\ell}}= & X_{\ell}\left(y_{\ell, 1}\right) \cdots X_{k}\left(y_{k, 2(\ell+1-k)-1}\right) \cdots X_{2}\left(y_{2,2 \ell-3}\right) \\
& \times X_{1}\left(y_{1, \ell}\right) X_{2}\left(y_{2,2 \ell-2}\right) \cdots X_{k}\left(y_{k, 2(\ell+1-k)}\right) X_{\ell}\left(y_{\ell, 2}\right) .
\end{aligned}
$$

Здесь $X_{i}(y)=e^{y f_{i}}$ и $f_{i} \equiv f_{\alpha_{i}}$ - образующие, которые соответствуют простым корням. Подмножество $N_{+}^{(0)}$ элементов, допускающих такое представление, открыто в $N_{+}$. Действие (1.10) алгебры Ли $\mathfrak{s o}_{2 \ell+1}$ на $N_{+}$, которое рассматривалось п. 1.1.2, определяет действие алгебры Ли на $N_{+}^{(0)}$. Следующее предложение дает явное описание этого действия на пространстве $V_{\mu}$, которое рассматривается как пространство эквивариантных функций на $N_{+}^{(0)}$.

ПредложениЕ 1.4. Следующие дифференциалъные оператори определяют реализачию представления основной серии $\pi_{\mu}$ алгебры $\mathscr{U}\left(\mathfrak{s o}_{2 \ell+1}\right)$ в терминах факторизачионной параметризачии на $N_{+}^{(0)}$ :

$$
\begin{aligned}
& F_{1}=\frac{\partial}{\partial y_{1, \ell}}+\sum_{n=1}^{\ell-1}\left\{\prod_{j=n}^{\ell-1} \frac{y_{2,2 i}}{y_{2,2 i-1}}\left(\frac{\partial}{\partial y_{1, n}}-\frac{\partial}{\partial y_{1, n+1}}\right)\right. \\
& \left.+2 \frac{y_{2,2(n-1)}}{y_{1, n}} \prod_{i=n+1}^{\ell-1} \frac{y_{2,2 i}}{y_{2,2 i-1}}\left(\frac{\partial}{\partial y_{2,2 n-1}}-\frac{\partial}{\partial y_{2,2 n}}\right)\right\} \\
& F_{k}=\frac{\partial}{\partial y_{k, 2(\ell+1-k)}}+\left(1-\delta_{k, \ell}\right) \sum_{n=1}^{\ell-k}\left\{\prod_{i=n}^{\ell-k} \frac{y_{k+1,2 i}}{y_{k+1,2 i-1}} \frac{y_{k, 2(i+1)-1}}{y_{k, 2(i+1)}}\left(\frac{\partial}{\partial y_{k, 2 n}}-\frac{\partial}{\partial y_{k, 2 n+1}}\right)\right. \\
& +\frac{y_{k+1,2 n}}{y_{k, 2(n+1)}} \prod_{i=n+1}^{\ell-k} \frac{y_{k+1,2 i}}{y_{k+1,2 i-1}} \frac{y_{k, 2(i+1)-1}}{y_{k, 2(i+1)}} \\
& \left.\times\left(\frac{\partial}{\partial y_{k+1,2 n-1}}-\frac{\partial}{\partial y_{k+1,2 n}}\right)\right\}, \quad k=2, \ldots, \ell, \\
& H_{k}=\left\langle\mu, \alpha_{k}^{\vee}\right\rangle+\sum_{i=1}^{\ell} a_{k, i} \sum_{j=1}^{n_{i}} y_{i, j} \frac{\partial}{\partial y_{i, j}}, \quad 1 \leqslant k \leqslant \ell, \\
& E_{1}=\sum_{n=1}^{\ell} y_{1, n}\left\{-\left\langle\mu, \alpha_{1}^{\vee}\right\rangle+\sum_{j=1}^{2 n-3} 2 y_{2, j} \frac{\partial}{\partial y_{2, j}}-2 \sum_{j=1}^{n-1} y_{1, j} \frac{\partial}{\partial y_{1, j}}-y_{1, n} \frac{\partial}{\partial y_{1, n}}\right\} \\
& E_{2}=\sum_{n=1}^{2(\ell-1)} y_{2, n}\left\{-\left\langle\mu, \alpha_{2}^{\vee}\right\rangle+\sum_{j=1}^{\alpha(n)+1} y_{1, j} \frac{\partial}{\partial y_{1, j}}-2 \sum_{j=1}^{n-1} y_{2, j} \frac{\partial}{\partial y_{2, j}}\right. \\
& \left.+\sum_{j=1}^{2 \beta(n)-3} y_{3, j} \frac{\partial}{\partial y_{3, j}}-y_{2, n} \frac{\partial}{\partial y_{2, n}}\right\}
\end{aligned}
$$




$$
\begin{aligned}
E_{k}=\sum_{n=1}^{2(\ell+1-k)} y_{k, n}\{ & -\left\langle\mu, \alpha_{k}^{\vee}\right\rangle+\sum_{j=1}^{2 \alpha(n)+2} y_{k-1, j} \frac{\partial}{\partial y_{k-1, j}}-2 \sum_{j=1}^{n-1} y_{k, j} \frac{\partial}{\partial y_{k, j}} \\
& \left.+\sum_{j=1}^{2 \beta(n)-3} y_{k+1, j} \frac{\partial}{\partial y_{k+1, j}}-y_{k, n} \frac{\partial}{\partial y_{k, n}}\right\}, \quad k=3, \ldots, \ell,
\end{aligned}
$$

где $\pi_{\mu}\left(e_{i}\right)=E_{i}, \pi_{\mu}\left(f_{i}\right)=F_{i}, \pi_{\mu}\left(h_{i}\right)=H_{i}, i=1, \ldots, \ell, n_{1}=\ell, n_{k}=2(\ell+1-k)$ при $1<k \leqslant \ell, a_{i j}$ - элементы матрицы Картана и предполагается, что члены, содержащие $y_{i, j}$ с индексами не из множества $\{1 \leqslant i, j \leqslant \ell\}$, опущены. Также мы используем обозначения $\alpha(n):=[n / 2], \beta(n):=[(n+1) / 2]$.

Доказательство дано в п. 2.4.2 части 2.

Левый и правый векторы Уиттекера определены посредством (1.7) и (1.8); в факторизационной параметризации они имеют следующий вид.

Лемма 1.5. Левый и правый векторы Уиттекера задаются выражениями

$$
\begin{aligned}
\psi_{R}(y)= & \exp \left\{-\left(\sum_{n=1}^{\ell} y_{1, n}+\sum_{k=2}^{\ell} \sum_{n=1}^{n_{k}} y_{k, n}\right)\right\} \\
\psi_{L}(y)=( & \left.\prod_{n=1}^{\ell} y_{1, n} \prod_{i=2}^{\ell} \prod_{n=1}^{n_{i} / 2} y_{i, 2 n-1}\right)^{\left\langle\mu, \alpha_{1}^{\vee}\right\rangle} \\
& \times \prod_{k=2}^{\ell}\left(\prod_{n=2}^{\ell} y_{1, n}^{2} \prod_{i=k+1}^{\ell} \prod_{n=1}^{n_{i} / 2} y_{i, 2 n-1}^{2} \prod_{i=2}^{k} \prod_{n=1}^{n_{i} / 2} y_{i, 2 n-1} y_{i, 2 n}\right)^{\left\langle\mu, \alpha_{k}^{\vee}\right\rangle} \\
& \times \exp \left\{-\left(\sum_{n=1}^{\ell} \frac{1}{y_{1, n}}\left(1+\frac{y_{2,2(n-1)}}{y_{2,2(n-1)-1}}\right) \prod_{i=n+1}^{\ell} \frac{y_{2,2(i-1)}}{y_{2,2(i-1)-1}}\right.\right. \\
& \left.\left.+\sum_{k=2}^{\ell} \sum_{n=1}^{n_{k} / 2} \frac{1}{y_{k, 2 n}}\left(1+\frac{y_{k+1,2(n-1)}}{y_{k+1,2(n-1)-1}}\right) \prod_{i=n+1}^{n_{k} / 2} \frac{y_{k+1,2(i-1)}}{y_{k+1,2(i-1)-1}} \frac{y_{k, 2 i-1}}{y_{k, 2 i}}\right)\right\},
\end{aligned}
$$

где $n_{1}=\ell$ u $n_{k}=2(\ell+1-k), k=2, \ldots, \ell$.

Доказательство дано в п. 2.3.2 части 2 .

Используя (1.12) и (1.21), мы получаем интегральное представление для $\mathfrak{s o}_{2 \ell+1}$-функций Уиттекера в факторизационной параметризации.

Tеорема 1.4. Собственные функции (1.12) $\mathfrak{s o}_{2 \ell+1}$-цепочки Тоды допускают следующее интегральное представление:

$$
\begin{gathered}
\Psi_{\lambda_{1}, \ldots, \lambda_{\ell}}^{B_{\ell}}\left(x_{1}, \ldots, x_{\ell}\right)=e^{\imath \lambda_{1} x_{1}+\cdots+\imath \lambda_{\ell} x_{\ell}} \int_{C} \prod_{i=1}^{\ell} \prod_{k=1}^{n_{i}} \frac{d y_{i, k}}{y_{i, k}}\left(\prod_{n=1}^{\ell} y_{1, n} \prod_{i=2}^{\ell} \prod_{n=1}^{n_{i} / 2} y_{i, 2 n-1}\right)^{2 \imath \lambda_{1}} \\
\times \prod_{k=2}^{\ell}\left(\prod_{n=2}^{\ell} y_{1, n}^{2} \prod_{i=k+1}^{\ell} \prod_{n=1}^{n_{i} / 2} y_{i, 2 n-1}^{2} \prod_{i=2}^{k} \prod_{n=1}^{n_{i} / 2} y_{i, 2 n-1} y_{i, 2 n}\right)^{\left(\lambda_{k}-\lambda_{k-1}\right)}
\end{gathered}
$$




$$
\begin{aligned}
& \times \exp \left\{-\left(\sum_{n=1}^{\ell} \frac{1}{y_{1, n}}\left(1+\frac{y_{2,2(n-1)}}{y_{2,2(n-1)-1}}\right) \prod_{i=n+1}^{\ell} \frac{y_{2,2(i-1)}}{y_{2,2(i-1)-1}}\right.\right. \\
& +\sum_{k=2}^{\ell} \sum_{n=1}^{n_{k} / 2} \frac{1}{y_{k, 2 n}}\left(1+\frac{y_{k+1,2(n-1)}}{y_{k+1,2(n-1)-1}}\right) \prod_{i=n+1}^{n_{k} / 2} \frac{y_{k+1,2(i-1)}}{y_{k+1,2(i-1)-1}} \frac{y_{k, 2 i-1}}{y_{k, 2 i}} \\
& \left.\left.+e^{x_{1}} \sum_{n=1}^{\ell} y_{1, n}+\sum_{k=2}^{\ell} e^{x_{k}-x_{k-1}} \sum_{n=1}^{n_{k}} y_{k, n}\right)\right\}
\end{aligned}
$$

где $n_{1}=\ell, n_{k}=2(\ell+1-k), k=2, \ldots, \ell, u C \subset N_{+}-$некомпактное подмногообразие половинной размерности, для которого подынтегральное выражение экспоненииально убъвает на границе и на бесконечности. В частности, в качестве $C$ можно взять малую дебормацию подмножества $\mathbb{R}_{+}^{\ell^{2}}$ в $\mathbb{C}^{\ell^{2}}$, обеспечиваюшую сходимость интеграла.

Доказательство приведено в п. 2.3.2 части 2.

ПримеР 1.2. Пусть $\ell=2$. В этом случае общая формула (1.74) приобретает вид

$$
\begin{aligned}
& \Psi_{\lambda_{1}, \lambda_{2}}^{B_{2}}\left(x_{1}, x_{2}\right)=e^{\imath \lambda_{1} x_{1}+\imath \lambda_{2} x_{2}} \int_{C} \prod_{i, k=1}^{2} \frac{d y_{i, k}}{y_{i, k}}\left(y_{1,1} y_{2,1} y_{1,2}\right)^{2 \imath \lambda_{1}}\left(y_{2,1} y_{1,2}^{2} y_{2,2}\right)^{\imath \lambda_{2}-\imath \lambda_{1}} \\
& \quad \times \exp \left\{-\left\{\frac{1}{y_{1,2}}+\frac{y_{2,2}}{y_{2,1}}\left(\frac{1}{y_{1,1}}+\frac{1}{y_{1,2}}\right)\right\}-\frac{1}{y_{2,2}}\right. \\
& \left.\quad-e^{x_{1}}\left(y_{1,1}+y_{1,2}\right)-e^{x_{2}-x_{1}}\left(y_{2,1}+y_{2,2}\right)\right\}
\end{aligned}
$$

где в качестве $C$ можно выбрать малую деформацию $\mathbb{R}_{+}^{4}$ в $\mathbb{C}^{4}$, обеспечивающую сходимость интеграла.

1.3.2. $\mathfrak{s o}_{2 \ell+1}$-функция Уиттекера: модифицированная факторизационная параметризация. В этом пункте мы определяем модифицированную факторизационную параметризацию на открытом подмножестве $N_{+}^{(0)} \subset \mathrm{SO}(2 \ell+1)$. Мы используем эту параметризацию для построения интегральных представлений $\mathfrak{s o}_{2 \ell+1}$-функций Уиттекера. В отличие от описанных выше интегральных представлений эти представления имеют простую рекурсивную структуру относительно ранга $\ell$ и могут быть описаны в сугубо комбинаторных терминах с использованием подходящих графов. Тем самым, эти представления можно рассматривать как обобщение интегрального представления Гивенталя на случай алгебры Ли $\mathfrak{s o}_{2 \ell+1}$.

Имеется реализация тавтологического представления $\pi: \mathfrak{s o}_{2 \ell+1} \rightarrow \operatorname{End}\left(\mathbb{C}_{2 \ell+1}\right)$, для которого образующие Шевалле, отвечающие борелевской (соответственно картановской) подалгебре $\mathfrak{s o}_{2 \ell+1}$, переходят в верхнетреугольные (соответственно диагональные) матрицы. Это определяет вложение $\mathfrak{s o}_{2 \ell+1} \subset \mathfrak{g l}_{2 \ell+1}$, для которого борелевская (соответственно картановская) подалгебра отображается в борелевскую (соответственно картановскую) подалгебру (см., например, [17]). 
Для того чтобы определить соответствующее вложение групп, рассмотрим инволюцию на GL $(2 \ell+1)$ :

$$
g \longmapsto g^{*}:=\dot{W}_{0}^{-1}\left(g^{-1}\right)^{t} \dot{W}_{0},
$$

где $a^{t}$ индуцировано стандартным транспонированием матрицы $a$ и $\dot{W}_{0}-$ подъем элемента максимальной длины $W_{0}$ группы Вейля $S_{2 \ell+1}$ алгебры $\mathfrak{g l}_{2 \ell+1}$. В матричной форме это может быть записано как

$$
\pi\left(\dot{W}_{0}\right)=S J
$$

где $S=\operatorname{diag}(1,-1, \ldots, 1,-1,1)$ и $J=\left\|J_{i, j}\right\|=\left\|\delta_{i+j, 2 \ell+2}\right\|$. Ортогональная группа $G=\mathrm{SO}(2 \ell+1)$ может быть определена как подгруппа в $\mathrm{GL}(2 \ell+1)$ :

$$
\mathrm{SO}(2 \ell+1)=\left\{g \in \mathrm{GL}(2 \ell+1): g^{*}=g\right\} .
$$

Пусть $\epsilon_{i, j}$ - элементарные $(2 \ell+1) \times(2 \ell+1)$-матрицы с единицей на месте $(i, j)$ и нулями на прочих местах. Рассмотрим матрицы

$$
\begin{aligned}
U_{n}=\sum_{i=1}^{\ell-n}\left(\epsilon_{i, i}+\epsilon_{2 \ell+2-i, 2 \ell+2-i}\right)+\sum_{i=1}^{n} \epsilon_{\ell-n+i, \ell-n+i}+e^{-z_{n, 1}} \epsilon_{\ell+1, \ell+1} \\
\quad+e^{z_{n, 1}} \epsilon_{\ell+2, \ell+2}+\sum_{i=1}^{n-1} e^{-z_{n, i+1}} \epsilon_{\ell+1+i, \ell+1+i}, \\
\widetilde{U}_{n}=U_{n}+\sum_{i=1}^{n-1}\left(1+\delta_{i, 1}\right) e^{-x_{n-1, i}} \epsilon_{\ell+1+i, \ell+2+i}
\end{aligned}
$$

И

$$
\begin{aligned}
& V_{n}=\sum_{i=1}^{\ell-n}\left(\epsilon_{i, i}+\epsilon_{2 \ell+2-i, 2 \ell+2-i}\right)+\sum_{i=1}^{n} e^{x_{n, i}} \epsilon_{\ell-1+i, \ell-1+i}+e^{-x_{n, 1}} \epsilon_{\ell+1, \ell+1} \\
& \quad+\sum_{i=1}^{n} \epsilon_{\ell+i+1, \ell+i+1} \\
& \widetilde{V}_{n}=V_{n}+2 e^{-z_{n, 1}} \epsilon_{\ell, \ell+1}+\sum_{i=1}^{n-1} e^{z_{n, i+1}} \epsilon_{\ell-i, \ell+1-i}
\end{aligned}
$$

при $n=2, \ldots, \ell$,

$$
\begin{aligned}
Z_{k} & =\sum_{i=1}^{\ell-1}\left(\epsilon_{i, i}+\epsilon_{2 \ell+2-i, 2 \ell+2-i}\right)+\epsilon_{\ell+1, \ell+1}+e^{-z_{n, 1}} \epsilon_{\ell, \ell}+e^{z_{n, 1}} \epsilon_{\ell+2, \ell+2}, \\
R_{k} & =\sum_{i=1}^{2 \ell+1} \epsilon_{i, i}+2 e^{2 x_{n, 1}} \epsilon_{\ell, \ell+2}
\end{aligned}
$$

при $k=1, \ldots, \ell$ и

$$
\begin{aligned}
U_{1} & =\sum_{i=1}^{\ell-1} \epsilon_{i, i}+e^{-z_{1,1}} \epsilon_{\ell, \ell}+e^{z_{1,1}} \epsilon_{\ell+1, \ell+1}+\sum_{i=\ell+2}^{2 \ell+1} \epsilon_{i, i}, \\
\widetilde{U}_{1} & =\sum_{i=1}^{\ell-1} \epsilon_{i, i}+e^{-z_{1,1}} \epsilon_{\ell, \ell}+e^{z_{1,1}} \epsilon_{\ell+1, \ell+1}+\sum_{i=\ell+2}^{2 \ell+1} \epsilon_{i, i}+2 e^{x_{1,1}} \epsilon_{\ell, \ell+1},
\end{aligned}
$$




$$
\begin{aligned}
& V_{1}=\sum_{i=1}^{\ell} \epsilon_{i, i}+e^{-z_{1,1}} \epsilon_{\ell+1, \ell+1}+e^{z_{1,1}} \epsilon_{\ell+2, \ell+2}+\sum_{i=\ell+3}^{2 \ell+1} \epsilon_{i, i} \\
& \widetilde{V}_{1}=U_{1}^{*}=\sum_{i=1}^{\ell} \epsilon_{i, i}+e^{-z_{1,1}} \epsilon_{\ell+1, \ell+1}+e^{z_{1,1}} \epsilon_{\ell+2, \ell+2}+\sum_{i=\ell+3}^{2 \ell+1} \epsilon_{i, i}+2 e^{x_{1,1}} \epsilon_{\ell+1, \ell+2},
\end{aligned}
$$

где $x_{\ell, k}=0$ при $k=1, \ldots, \ell$. Заметим, что $\widetilde{V}_{i}, \widetilde{U}_{i}$ можно рассматривать как внедиагональные деформации $V_{i}, U_{i}$. Сейчас мы готовы определить модифицированное факторизационное представление на $N_{+} \subset \mathrm{SO}(2 \ell+1)$.

Теорема 1.5. (i) Образ типичного унипотентного элемента $v^{B_{\ell}} \in N_{+}$nри тавтологическом представлении $\pi: \mathfrak{s o}_{2 \ell+1} \rightarrow \operatorname{End}\left(\mathbb{C}^{2 \ell+1}\right)$ можно записать $в$ виде

$$
\pi\left(v^{B_{\ell}}\right)=\mathfrak{X}_{1} \mathfrak{X}_{2} \cdots \mathfrak{X}_{\ell}, \quad \mathfrak{X}_{n}=\pi\left(\mathfrak{X}_{B_{n-1}}^{B_{n}}\right), \quad n=1, \ldots, \ell,
$$

¿əe

$$
\begin{aligned}
& \mathfrak{X}_{1}=\widetilde{U}_{1} U_{1}^{-1} Z_{1} R_{1} Z_{1}^{-1} \widetilde{V}_{1} V_{1}^{-1}, \\
& \mathfrak{X}_{n}=\widetilde{U}_{n} U_{n}^{-1} Z_{n} R_{n-1} Z_{n}^{-1}\left[\widetilde{U}_{n} U_{n}^{-1}\right]^{*} \cdot\left[\widetilde{V}_{n} V_{n}^{-1}\right]^{*} Z_{n} R_{n} Z_{n}^{-1} \widetilde{V}_{n} V_{n}^{-1}, \quad n=2, \ldots, \ell,
\end{aligned}
$$

$u x_{\ell, k}=0$ nрu $k=1, \ldots, \ell$.

(ii) Формуль (1.87), (1.88) определяют параметризацию множества полностъю неотрицательных унипотентных элементов $N_{+}^{(0)} \subset N_{+}$.

ДокАЗАТЕЛьство. Пусть $v^{B_{\ell}}(y)$ - параметризация на открытом подмножестве в $N_{+}$, соответствующая $(1.67),(1.68)$, и пусть $\widetilde{X}_{k}(y)=e^{y \epsilon_{\ell+1-k, \ell+2-k}}$, $k=2, \ldots, \ell,-$ унипотентные элементы в $\mathrm{GL}(2 \ell+1)$, отвечающие простым корням $\alpha_{1}, \ldots, \alpha_{\ell}$. Действие инволюции * определяется как $\widetilde{X}_{i}(y)^{*}=\widetilde{X}_{\ell+i}(y)$. Вложим элементарный унипотентный элемент $X_{i}(y)$ группы $\mathrm{SO}(2 \ell+1)$ в $\mathrm{GL}(2 \ell+1)$ следующим образом:

$$
X_{1}(y)=e^{2 y\left(\epsilon_{\ell, \ell+1}+\epsilon_{\ell+1, \ell+2}\right)}, \quad X_{k}(y)=\widetilde{X}_{i}(y)^{*} \widetilde{X}_{i}(y), \quad k=2, \ldots, \ell .
$$

Тем самым мы отображаем произвольный регулярный унипотентный элемент $v^{B_{\ell}}$ в унипотентную подгруппу в $\mathrm{GL}(2 \ell+1)$. Сделаем следующую замену переменных:

$$
\begin{aligned}
y_{1,1} & =e^{x_{1,1}-z_{1,1}}, & & \\
y_{1, k} & =e^{x_{k-1,1}-z_{k, 1}}+e^{x_{k, 1}-z_{k, 1}}, & & k=2, \ldots, \ell, \\
y_{k, 2 r-1} & =e^{z_{k+r-1, k}-x_{k+r-2, k-1}}, & & r=1, \ldots, \ell+1-k, \\
y_{k, 2 r} & =e^{z_{k+r-1, k}-x_{k+r-1, k-1}}, & &
\end{aligned}
$$

где предполагаются выполненными условия $x_{\ell, k}=0, k=1, \ldots, \ell$. Используя элементарные операции, несложно проверить, что после замены переменных (1.89) образ $\pi\left(v^{B_{\ell}}\right)$ элемента $v^{B_{\ell}}$, определенный посредством (1.67), (1.68), преобразуется в $(1.87),(1.88)$, и это приводит к параметризации на $N_{+}^{(0)} \subset N_{+}$. Теорема 1.5 доказана. 
Модифицированная факторизационная параметризация унипотентной подгруппы $N_{+} \subset \mathrm{SO}(2 \ell+1)$ определяет специальную реализацию представления $\pi_{\mu}$ основной серии алгебры $\mathscr{U}\left(\mathfrak{s o}_{2 \ell+1}\right)$ дифференциальными операторами. Ее можно получить, применяя замену переменных (1.89) к представлению из предложения 1.4. Мы будем называть эту реализацию представлением Гаусса-Гивенталя.

ПРеДлОЖенИЕ 1.5. Следующие дифференииалъные операторы определяют представление $\pi_{\mu}$ алгебры Ли $\mathfrak{s o}_{2 \ell+1}$ в пространстве $V_{\mu}$ в терминах модифииированной факторизачионной параметризачии:

$$
\begin{aligned}
& F_{1}=-e^{z_{1}} \frac{\partial}{\partial z_{1,1}}-2 \sum_{n=2}^{\ell} e^{z_{n, 1}}\left\{\frac{1}{2} \frac{\partial}{\partial z_{n, 1}}+\frac{e^{x_{n, 1}}}{e^{x_{n-1,1}}+e^{x_{n, 1}}} \frac{\partial}{\partial z_{n, 2}}\right. \\
& \left.+\sum_{k=1}^{n-1}\left(\frac{\partial}{\partial z_{k, 1}}+\frac{\partial}{\partial z_{k, 2}}+\frac{\partial}{\partial x_{k, 1}}\right)\right\} \\
& F_{2}=\sum_{n=1}^{2 \ell-3} e^{x_{\beta(n)+1,2}-z_{\alpha(n)+2,2}}\left\{\frac{\partial}{\partial z_{1,1}}+\frac{\partial}{\partial x_{1,1}}+\sum_{k=1}^{\beta(n)}\left(\frac{\partial}{\partial z_{k+1,1}}+\frac{\partial}{\partial z_{k+1,2}}-\frac{\partial}{\partial z_{k+1,3}}\right)\right. \\
& \left.+\sum_{k=1}^{\alpha(n)+1}\left(\frac{\partial}{\partial x_{k, 1}}-\frac{\partial}{\partial x_{k, 2}}\right)+(-1)^{n} \frac{e^{x_{\beta(n)+1,1}}}{e^{x_{\alpha(n)+1,1}}+e^{x_{\alpha(n)+2,1}}} \frac{\partial}{\partial z_{\alpha(n)+2,1}}\right\}, \\
& F_{i}=\sum_{n=1}^{2(\ell-i)+1} e^{x_{\beta(n)+i-1, i}-z_{\alpha(n)+i, i}}\left\{\frac{\partial}{\partial z_{i-1, i-1}}+\frac{\partial}{\partial x_{i-1, i-1}}\right. \\
& +\sum_{k=1}^{\alpha(n)}\left(\frac{\partial}{\partial x_{i+k-1, i-1}}-\frac{\partial}{\partial x_{i+k-1, i}}\right) \\
& \left.+\sum_{k=1}^{\beta(n)}\left(\frac{\partial}{\partial z_{i+k-1, i}}-\frac{\partial}{\partial z_{i+k-1, i+1}}\right)\right\}, \quad i=3, \ldots, \ell \\
& H_{k}=\left\langle\mu, \alpha_{k}^{\vee}\right\rangle+\sum_{n=1}^{\ell}(-1)^{\delta_{n, 1}} a_{k, n} \sum_{i=n}^{\ell} \frac{\partial}{\partial z_{i, n}}, \quad k=1, \ldots, \ell, \\
& E_{1}=-\sum_{n=1}^{2 \ell-1} e^{x_{\beta(n), 1}-z_{\alpha(n)+1,1}}\left\{2 \mu_{1}+2 \sum_{k=1}^{\alpha(n)} \frac{\partial}{\partial x_{k, 1}}+(-1)^{n} \frac{\partial}{\partial z_{\alpha(n)+1,1}}\right\} \text {, } \\
& E_{2}=\sum_{n=1}^{2(\ell-1)} e^{z_{\beta(n)+1,2}-x_{\alpha(n)+1,1}}\left\{\mu_{1}-\mu_{2}+\sum_{k=1}^{\beta(n)}\left(\frac{\partial}{\partial x_{k, 1}}-\frac{\partial}{\partial x_{k, 2}}\right)-\sum_{k=1}^{\alpha(n)} \frac{\partial}{\partial z_{k+1,2}}\right\}, \\
& E_{i}=\sum_{n=1}^{2(\ell+1-i)} e^{z_{\beta(n)+i-1, i}-x_{\alpha(n)+i-1, i-1}}\left\{\mu_{i-1}-\mu_{i}+\sum_{k=0}^{\alpha(n)}\left(\frac{\partial}{\partial z_{k+i-1, i-1}}-\frac{\partial}{\partial z_{k+i-1, i}}\right)\right. \\
& \left.+\sum_{k=0}^{\beta(n)-1}\left(\frac{\partial}{\partial x_{k+i-1, i-1}}-\frac{\partial}{\partial x_{k+i-1, i}}\right)\right\}, \quad i=3, \ldots, \ell .
\end{aligned}
$$


Здесъ $E_{i}=\pi_{\lambda}\left(e_{i}\right), F_{i}=\pi_{\lambda}\left(f_{i}\right), H_{i}=\pi_{\lambda}\left(h_{i}\right), x_{\ell, k}=0, k=1, \ldots, \ell$, u дифберенцирования по $x_{i, k}, z_{i, k}, i<k$, и по $x_{\ell, n}, n=1, \ldots, \ell$, мы опускаем. Также мы используем обозначения $\alpha(n):=[n / 2], \beta(n):=[(n+1) / 2]$.

Сейчас мы явно выпишем матричный элемент (1.12) в представлении Гаусса-Гивенталя. Векторы Уиттекера $\psi_{R}$ и $\psi_{L}$ в этом представлении удовлетворяют системам дифференциальных уравнений (1.7) и (1.8):

$$
F_{i} \psi_{R}(x)=-\psi_{R}(x), \quad E_{i} \psi_{L}(x)=-\psi_{L}(x), \quad i=1, \ldots, \ell
$$

Их решения имеют следующий вид.

ЛЕмма 1.6. Функиии

$$
\begin{gathered}
\psi_{L}(x, z)=e^{2 \mu_{1} x_{1,1}} \prod_{n=2}^{\ell}\left(e^{x_{n, 1}}+e^{x_{n-1,1}}\right)^{2 \mu_{n}} \\
\times \prod_{n=1}^{\ell} \exp \left\{-\mu_{n}\left(\sum_{i=1}^{n} x_{n, i}+2 z_{n, 1}-2 \sum_{i=2}^{n} z_{n, i}+\sum_{i=1}^{n-1} x_{n-1, i}\right)\right\} \\
\times \exp \left\{-\left(\sum_{k=1}^{\ell} e^{z_{k, 1}}+\sum_{k=2}^{\ell} e^{x_{k, k}-z_{k, k}}+\sum_{k=2}^{\ell} \sum_{n=k+1}^{\ell}\left(e^{x_{n-1, k}-z_{n, k}}+e^{x_{n, k}-z_{n, k}}\right)\right)\right\} \\
\psi_{R}(x, z)=\exp \left\{-\left(e^{x_{1,1}-z_{1,1}}+\sum_{n=2}^{\ell}\left(e^{x_{n-1,1}-z_{n, 1}}+e^{x_{n, 1}-z_{n, 1}}\right)\right)\right. \\
\left.+\sum_{k=2}^{\ell} \sum_{n=k}^{\ell}\left(e^{z_{n, k}-x_{n-1, k-1}}+e^{z_{n, k}-x_{n, k-1}}\right)\right\}
\end{gathered}
$$

являются решениями линейных дифференциалъных уравнений (1.7), (1.8). Здесь $x_{\ell, k}=0$ при $k=1, \ldots, \ell$ u $\mu_{k}=\imath \lambda_{k}-\rho_{k}$, где $\rho_{k}=(2 k-1) / 2$.

Мы готовы предъявить интегральное представление для спаривания (1.12) в терминах модифицированных параметров факторизации. Для того чтобы получить явное выражение для подынтегральной функции, используется тот же тип разложения элемента максимального тора, что и в случае $\mathfrak{g l}_{\ell+1}$ :

$$
e^{-H_{x}}=\pi_{\lambda}\left(\exp \left(-\sum_{i=1}^{\ell}\left\langle\omega_{i}, x\right\rangle h_{i}\right)\right)=e^{H_{L}} e^{H_{R}}
$$

где

$$
\begin{aligned}
-H_{x}=H_{L}+H_{R} & =-\sum_{i=1}^{\ell}\left\langle\omega_{i}, x\right\rangle\left\langle\mu, \alpha_{i}^{\vee}\right\rangle \\
& +x_{\ell, 1} \sum_{n=1}^{\ell} \frac{\partial}{\partial z_{n, 1}}+\sum_{k=1}^{\ell-1}\left(x_{\ell, i}-x_{\ell, i+1}\right) \sum_{n=k}^{\ell} \frac{\partial}{\partial z_{n, k}}
\end{aligned}
$$


и

$$
\begin{gathered}
H_{L}=\sum_{k=1}^{\ell-1} \sum_{n=1}^{k} x_{\ell, n} \frac{\partial}{\partial x_{k, n}}+\sum_{k=2}^{\ell} \sum_{n=2}^{k} x_{\ell, n} \frac{\partial}{\partial z_{k, n}} \\
H_{R}=-H_{x}-H_{L} .
\end{gathered}
$$

Напомним, что дифференциальный оператор $H_{L}$ действует на левый вектор, a $H_{R}$ действует на правый вектор в (1.12). Учитывая лемму 1.6, мы получаем следующую теорему.

Теорема 1.6. Собственные функиии $\mathfrak{s o}_{2 \ell+1}$-цепочки Тодъ (1.12) допускают интегральное представление:

$$
\Psi_{\lambda_{1}, \ldots, \lambda_{\ell}}^{B_{\ell}}\left(x_{\ell, 1}, \ldots, x_{\ell, \ell}\right)=\int_{C} \prod_{k=1}^{\ell-1} d \underline{x}_{k} \prod_{k=1}^{\ell} d \underline{z}_{k} e^{\mathscr{F}^{B_{\ell}}}
$$

где

$$
\begin{aligned}
\mathscr{F}^{B_{\ell}}= & -\imath \lambda_{1}\left(-x_{1,1}+2 z_{1,1}\right) \\
& -\imath \sum_{n=2}^{\ell} \lambda_{n}\left(\sum_{i=1}^{n} x_{n, i}+2 z_{n, 1}-2 \sum_{i=2}^{n} z_{n, i}+\sum_{i=1}^{n-1} x_{n-1, i}-2 \ln \left(e^{x_{n, 1}}+e^{x_{n-1,1}}\right)\right) \\
& -\left\{\sum_{n=1}^{\ell} e^{z_{n, 1}}+\sum_{k=2}^{\ell} \sum_{n=k+1}^{\ell}\left(e^{x_{n-1, k}-z_{n, k}}+e^{x_{n, k}-z_{n, k}}\right)\right. \\
& \left.+\sum_{n=k}^{\ell}\left(e^{z_{n, k}-x_{n-1, k-1}}+e^{z_{n, k}-x_{n, k-1}}\right)+\sum_{n=1}^{\ell} e^{x_{n, n}-z_{n, n}}\right\}
\end{aligned}
$$

и $x_{i}:=x_{\ell, i}, i=1, \ldots, \ell$. Здесъ $C \subset N_{+}-$некомпактное подмногообразие половинной размерности, на котором подынтегральное выражение экспоненииально убъвает на границе и на бесконечности. В частности, область интегрирования можно положить равной малой деформации подпространства $\mathbb{R}^{m} \subset \mathbb{C}^{m}, m=l\left(w_{0}\right)$, обеспечивающей сходимость интеграла.

Заметим, что интеграл (1.102) можно получить непосредственно из (1.74), используя следующую замену переменных:

$$
\begin{array}{rlrl}
y_{1,1} & =e^{-x_{\ell, 1}} e^{x_{1,1}-z_{1,1}}, \\
y_{1, k} & =e^{-x_{\ell, 1}}\left(e^{x_{k-1,1}-z_{k, 1}}+e^{x_{k, 1}-z_{k, 1}}\right), & & k=2, \ldots, \ell, \\
y_{k, 2 r-1} & =e^{-x_{\ell, k-1}-x_{\ell, k}} e^{z_{k+r-1, k}-x_{k+r-2, k-1}}, & & r=1, \ldots, \ell+1-k, \\
y_{k, 2 r} & =e^{-x_{\ell, k-1}-x_{\ell, k}} e^{z_{k+r-1, k}-x_{k+r-1, k-1}} . &
\end{array}
$$

ПримеР 1.3. При $\ell=2$ общая формула (1.102) принимает вид

$$
\begin{aligned}
\Psi_{\lambda_{1}, \lambda_{2}}^{B_{2}} & \left(x_{2,1}, x_{2,2}\right)=\int_{C} d z_{1,1} d x_{1,1} d z_{2,1} d z_{2,2} \\
& \times e^{-\imath \lambda_{1}\left(-x_{1,1}+2 z_{1,1}\right)-\imath \lambda_{2}\left(2 z_{2,1}-2 z_{2,2}+x_{1,1}+x_{2,1}+x_{2,2}\right)}\left(e^{x_{2,1}}+e^{x_{1,1}}\right)^{2 \imath \lambda_{2}} \\
& \times \exp \left\{-\left(e^{z_{1,1}}+e^{z_{2,1}}+e^{x_{2,2}-z_{2,2}}+e^{x_{1,1}-z_{1,1}}+e^{x_{1,1}-z_{2,1}}\right.\right. \\
& \left.\left.+e^{x_{2,1}-z_{2,1}}+e^{z_{2,2}-x_{1,1}}+e^{z_{2,2}-x_{2,1}}\right)\right\} .
\end{aligned}
$$


Имеется простое комбинаторное описание потенциала $\mathscr{F}^{B_{\ell}}$ на нулевом уровне спектра $\left\{\lambda_{i}=0\right\}$. А именно, он может быть представлен как сумма по всем стрелкам на следующей диаграмме:

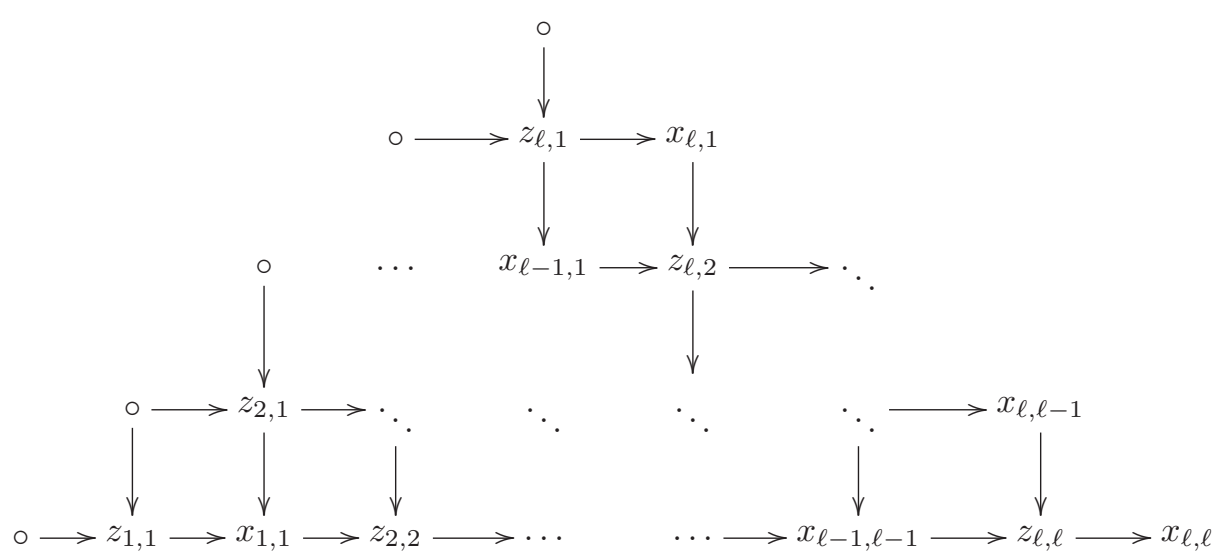

Здесь мы используем те же правила сопоставления переменных стрелкам, что и на диаграмме для типа $A_{\ell}$. Также сопоставим стрелкам $\circ \longrightarrow x$ функции $e^{x} / 2$.

Заметим, что диаграмма для типа $B_{\ell}$ может быть получена из диаграммы (1.51) для типа $A_{2 \ell}$ факторизацией. А именно, рассмотрим инволюцию

$$
*: X \longmapsto-\dot{W}_{0}^{-1} X^{t} \dot{W}_{0},
$$

где $W_{0}$ - элемент максимальной длины в группе Вейля $S_{2 \ell+1}$ системы корней $A_{2 \ell}$ и $X^{t}$ обозначает стандартное транспонирование. Соответствующее действие на модифицированных параметрах факторизации задано как

$$
*: x_{k, i} \longleftrightarrow-x_{k, k+1-i} .
$$

Это определяет факторизацию $A_{2 \ell}$-диаграммы, которая приводит к диаграмме типа $B_{\ell}$.

Можно получить аналог соотношений (1.52) следующим образом. Свяжем переменные $a_{k, i}, b_{k, i}, c_{k, i}, d_{k, i}$ со стрелками диаграммы Гивенталя:

$$
\begin{gathered}
a_{k, 1}=b_{k, 1}=\frac{1}{2} e^{z_{k, 1}}, \quad k=1, \ldots, \ell ; \\
a_{k, i}=e^{z_{k, i}-x_{k-1, i-1}}, \quad b_{k, i}=e^{z_{k, i}-x_{k, i-1}}, \quad 1<i \leqslant k \leqslant \ell ; \\
c_{k, a}=e^{x_{k, a}-z_{k, a}}, \quad 1 \leqslant a \leqslant k \leqslant \ell ; \\
d_{m, j}=e^{x_{m, j}-z_{m+1, j}}, \quad 1 \leqslant j \leqslant m<\ell .
\end{gathered}
$$

Тогда имеют место следующие соотношения:

$$
\begin{gathered}
a_{k, 1}=b_{k, 1}, \quad 1 \leqslant k \leqslant \ell \\
d_{k, i} a_{k+1, i+1}=c_{k+1, i} b_{k+1, i+1}, \quad 1 \leqslant i \leqslant k<\ell-1, \\
b_{k, i} c_{k, i}=a_{k+1, i} d_{k, i}, \quad 1 \leqslant i \leqslant k<\ell-1, \\
b_{\ell, 1} c_{\ell, 1}=e^{x_{\ell, 1}}, \quad b_{\ell, i} c_{\ell, i}=e^{x_{\ell, i}-x_{\ell, i-1}}, \quad i=2, \ldots, \ell .
\end{gathered}
$$


Эти равенства можно рассматривать как соотношения между элементарными путями на диаграмме Гивенталя. Используя соотношения для более общих путей, которые вытекают из (1.108), можно определить торическое вырождение многообразий флагов для $\mathfrak{s o}_{2 \ell+1}$, которое обобщает результаты работы [5]. Заметим, что приведенная выше диаграмма совпадает с диаграммой Гельфанда-Цейтлина для алгебры Ли $\mathfrak{s o}_{2 \ell+1}$.

1.3.3. Рекурсия для $\mathfrak{s o}_{2 \ell+1}$-функций Уиттекера и $\mathscr{Q}$-оператор для $B_{\ell}^{(1)}$-цепочки Тоды. Интегральное представление (1.102) из теоремы 1.6 для $\mathfrak{s o}_{2 \ell+1}$ функций Уиттекера обладает замечательной рекурсивной структурой относительно ранга $\ell$. Нам будет удобно использовать следующее обозначение: $\underline{a}_{n}=$ $\left(a_{n, 1}, \ldots, a_{n, n}\right)$ при $n=1, \ldots, \ell$. Введем интегральные операторы $Q_{B_{n-1}}^{B_{n}}, n=$ $2, \ldots, \ell$, с ядрами $Q_{B_{n-1}}^{B_{n}}\left(\underline{x}_{n}, \underline{x}_{n-1} ; \lambda_{n}\right)$, определяемые следующим образом:

$$
\begin{aligned}
& Q_{B_{n-1}}^{B_{n}}\left(\underline{x}_{n}, \underline{x}_{n-1} ; \lambda_{n}\right)=\int d \underline{z}_{n} Q_{B C_{n}}^{B_{n}}\left(\underline{x}_{n}, \underline{z}_{n}\right) Q_{B_{n-1}}^{B C_{n}}\left(\underline{z}_{n}, \underline{x}_{n-1}\right)\left(e^{x_{n, 1}}+e^{x_{n-1,1}}\right)^{2 \imath \lambda_{n}} \\
& \quad \times \exp \left\{-\imath \lambda_{n}\left(\sum_{i=1}^{n} x_{n, i}+2 z_{n, 1}-2 \sum_{i=2}^{n} z_{n, i}+\sum_{i=1}^{n-1} x_{n-1, i}\right)\right\},
\end{aligned}
$$

где

$$
\begin{aligned}
Q_{B C_{n}}^{B_{n}}\left(\underline{x}_{n}, \underline{z}_{n}\right) & =\exp \left\{-\frac{1}{2} e^{z_{n, 1}}-\sum_{i=1}^{n-1}\left(e^{x_{n, i}-z_{n, i}}+e^{z_{n, i+1}-x_{n, i}}\right)-e^{x_{n, n}-z_{n, n}}\right\}, \\
Q_{B_{n-1}}^{B C_{n}}\left(\underline{z}_{n}, \underline{x}_{n-1}\right) & =\exp \left\{-\frac{1}{2} e^{z_{n, 1}}-\sum_{i=1}^{n-1}\left(e^{x_{n-1, i}-z_{n, i}}+e^{z_{n, i+1}-x_{n-1, i}}\right)\right\} .
\end{aligned}
$$

При $n=1$ положим

$$
Q_{B_{0}}^{B_{1}}\left(x_{1,1} ; \lambda_{1}\right)=\Psi_{\lambda_{1}}^{B_{1}}\left(x_{1,1}\right)=\int d z_{1,1} e^{\imath \lambda_{1}\left(x_{1,1}-2 z_{1,1}\right)} \exp \left\{-\left(e^{z_{1,1}}+e^{x_{1,1}-z_{1,1}}\right)\right\} .
$$

ЗАмечАниЕ 1.1. Сделаем следующую замену переменных $\underline{z}_{n}$ под знаком интеграла в (1.109):

$$
\begin{aligned}
z_{n, 1} \longmapsto & -z_{n, 1}+\ln \left(e^{x_{n-1,1}}+e^{x_{n, 1}}\right), \\
z_{n, k} \longmapsto & -z_{n, k}+x_{n-1, k-1}+x_{n, k-1} \\
& +\ln \left(e^{x_{n-1, k}}+e^{x_{n, k}}\right)-\ln \left(e^{x_{n-1, k-1}}+e^{x_{n, k-1}}\right) \quad \text { при } \quad k=2, \ldots, n-1, \\
& \quad-z_{n, n}+x_{n-1, n-1}+x_{n, n-1}+x_{n, n}-\ln \left(e^{x_{n-1, n-1}}+e^{x_{n, n-1}}\right) .
\end{aligned}
$$

Это даст более простое интегральное представление оператора рекурсии

$$
\begin{aligned}
& Q_{B_{n-1}}^{B_{n}}\left(\underline{x}_{n}, \underline{x}_{n-1} ; \lambda_{n}\right)=\int d \underline{z}_{n} Q_{B C_{n}}^{B_{n}}\left(\underline{x}_{n}, \underline{z}_{n}\right) Q_{B_{n-1}}^{B C_{n}}\left(\underline{z}_{n}, \underline{x}_{n-1}\right) \\
& \times \exp \left\{\imath \lambda_{n}\left(\sum_{i=1}^{n} x_{n, i}+2 z_{n, 1}-2 \sum_{i=2}^{n} z_{n, i}+\sum_{i=1}^{n-1} x_{n-1, i}\right)\right\}
\end{aligned}
$$

и $\mathfrak{s o}_{2 \ell+1}$-волновой функции Тоды. 
Используя интегральные операторы $Q_{B_{n-1}}^{B_{n}}$, можно переписать интегральное представление (1.102) в рекурсивной форме.

Теорема 1.7. Собственная функиия $B_{\ell}$-цепочки Тоды может быть записана в виде

$$
\Psi_{\lambda_{1}, \ldots, \lambda_{\ell}}^{B_{\ell}}\left(x_{1}, \ldots, x_{\ell}\right)=\int_{C} \prod_{k=1}^{\ell-1} d \underline{x}_{k} \prod_{k=1}^{\ell} Q_{B_{k-1}}^{B_{k}}\left(\underline{x}_{k}, \underline{x}_{k-1} ; \lambda_{k}\right),
$$

или, эквивалентно,

$$
\Psi_{\lambda_{1}, \ldots, \lambda_{\ell}}^{B_{\ell}}\left(x_{1}, \ldots, x_{\ell}\right)=\int_{\mathscr{C}_{\ell}} d \underline{x}_{\ell-1} Q_{B_{\ell-1}}^{B_{\ell}}\left(\underline{x}_{\ell}, \underline{x}_{\ell-1} ; \lambda_{\ell}\right) \Psi_{\lambda_{1}, \ldots, \lambda_{\ell-1}}^{B_{\ell-1}}\left(\underline{x}_{\ell-1}\right),
$$

где $x_{i}:=x_{\ell, i}, i=1, \ldots, \ell, u C \subset N_{+}$- некомпактное подмногообразие половинной размерности, для которого подынтегральная функиия экспоненциально убъвает на возможных границах и на бесконечности.

Отметим, что, в отличие от интегральных представлений в случае $\mathfrak{g l}_{\ell+1}$, ядра операторов $Q_{B_{n-1}}^{B_{n}}, n=1, \ldots, \ell$, имеют сложный вид. Рассматривая функции Уиттекера на нулевом уровне спектра $\left\{\lambda_{i}=0\right\}$, мы приходим к новым интересным структурам. Как следует из (1.109), ядро оператора $Q_{B_{n-1}}^{B_{n}}$ задается сверткой двух ядер $Q_{B C_{n}}^{B_{n}}\left(\underline{x}_{n}, \underline{z}_{n}\right)$ и $Q_{B_{n-1}}^{B C_{n}}\left(\underline{z}_{n}, \underline{x}_{n-1}\right)$. Соответствующие интегральные операторы $Q_{B C_{n}}^{B_{n}}, Q_{B_{n-1}}^{B C_{n}}$ можно рассматривать как элементарные сплетения, связывающие цепочки Тоды для систем корней $B_{n}, B C_{n}$ и $B C_{n}, B_{n-1}$, при этом $B C_{\ell-\text { цепочка Тоды }}{ }^{1}$ определяется в терминах неприведенной системы корней $B C_{\ell}$ стандартным образом. Напомним конструкцию неприведенной системы корней $B C_{\ell}$. Система корней типа $B C_{\ell}$ может быть реализована в ортонормированном базисе $\left\{\epsilon_{i}\right\}$ пространства $\mathbb{R}^{\ell}$ как

$$
\alpha_{0}=2 \epsilon_{1}, \quad \alpha_{1}=\epsilon_{1}, \quad \alpha_{i+1}=\epsilon_{i+1}-\epsilon_{i}, \quad 1 \leqslant i \leqslant \ell-1,
$$

и соответствующая диаграмма Дынкина имеет вид

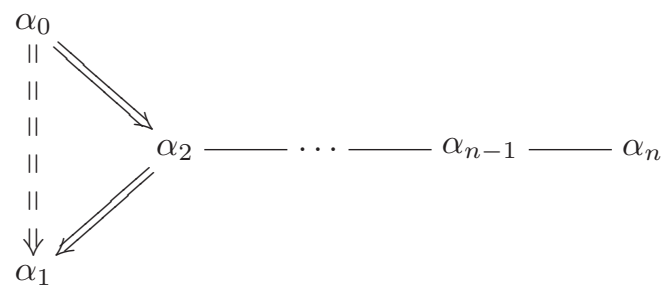

где первая слева вершина отвечает приведенному корню $\alpha_{1}=\epsilon_{1}$ и неприведенному корню $\alpha_{0}=2 \epsilon_{1}$. Например, квадратичный гамильтонов оператор $B C_{\ell}$-цепочки Тоды имеет вид

$$
\mathscr{H}_{2}^{B C_{\ell}}\left(\underline{x}^{(\ell)}\right)=-\frac{1}{2} \sum_{i=1}^{\ell} \frac{\partial^{2}}{\partial x_{i}^{2}}+\frac{1}{4}\left(e^{x_{1}}+\frac{1}{2} e^{2 x_{1}}\right)+\sum_{i=1}^{\ell-1} e^{x_{i+1}-x_{i}} .
$$

\footnotetext{
${ }^{1} B C_{\ell}$-цепочку Тоды можно рассматривать как наиболее общую форму $C_{\ell}$-цепочки Тоды (см., например, [32; замечание на с. 61]). Далее мы используем термин " $B C_{\ell}$-цепочка Тоды", для того чтобы отличать ее от более стандартной $C_{\ell}$-цепочки Тоды, которая рассматривается ниже.
} 
Интегральные операторы $Q_{B C_{n}}^{B_{n}}$ и $Q_{B_{n-1}}^{B C_{n}}$ сплетают гамильтоновы операторы цепочек Тоды для систем корней разных типов. Тем самым, для квадратичных гамильтонианов несложно непосредственно проверить следующие соотношения.

Предложение 1.6. 1. Операторъ $Q_{B C_{n}}^{B_{n}} u Q_{B_{n-1}}^{B C_{n}}$, определяемъе ядрами (1.110), (1.111), сплетают квадратичные гамильтонианы B- и ВС-цепочек Toды:

$$
\begin{aligned}
\mathscr{H}_{2}^{B C_{n}}\left(\underline{z}_{n}\right) Q_{B_{n-1}}^{B C_{n}}\left(\underline{z}_{n}, \underline{x}_{n-1}\right) & =Q_{B_{n-1}}^{B C_{n}}\left(\underline{z}_{n}, \underline{x}_{n-1}\right) \mathscr{H}_{2}^{B_{n-1}}\left(\underline{x}_{n-1}\right), \\
\mathscr{H}_{2}^{B_{n}}\left(\underline{x}_{n}\right) Q_{B C_{n}}^{B_{n}}\left(\underline{x}_{n}, \underline{z}_{n}\right) & =Q_{B C_{n}}^{B_{n}}\left(\underline{x}_{n}, \underline{z}_{n}\right) \mathscr{H}_{2}^{B C_{n}}\left(\underline{z}_{n}\right) .
\end{aligned}
$$

2. Интегральньй оператор $Q_{B_{n-1}}^{B_{n}}$ nри $\lambda_{n}=0$ сплетает гамильтониань $\mathscr{H}_{2}^{B_{n}} u \mathscr{H}_{2}^{B_{n-1}}$ :

$$
\mathscr{H}_{2}^{B_{n}}\left(\underline{x}_{n}\right) Q_{B_{n-1}}^{B_{n}}\left(\underline{x}_{n}, \underline{x}_{n-1} ; \lambda_{n}=0\right)=Q_{B_{n-1}}^{B_{n}}\left(\underline{x}_{n}, \underline{x}_{n-1} ; \lambda_{n}=0\right) \mathscr{H}_{2}^{B_{n-1}}\left(\underline{x}_{n-1}\right) .
$$

Ядро $Q_{B_{n-1}}^{B_{n}}\left(\underline{x}_{n}, \underline{x}_{n-1} ; \lambda_{n}=0\right)$ можно задать следующей поддиаграммой диаграммы Гивенталя для $\mathfrak{s o}_{2 \ell+1}$ :

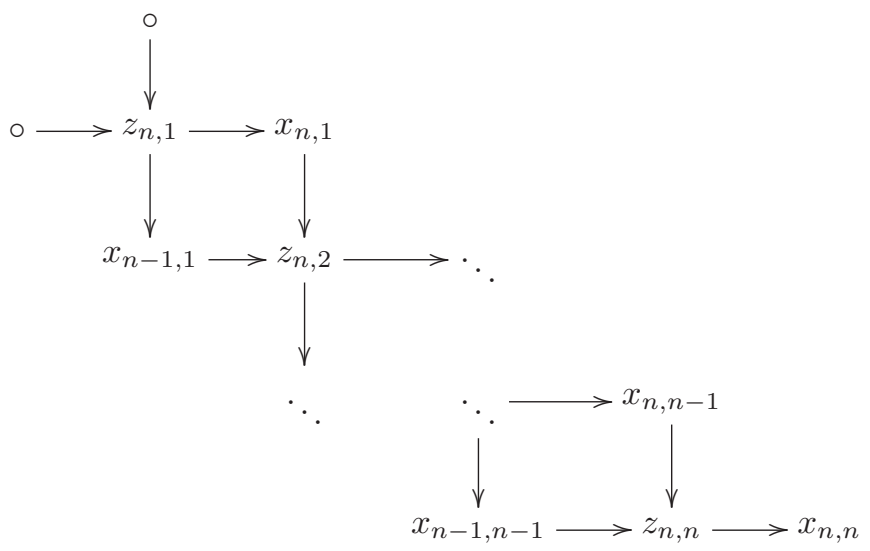

Здесь верхний и нижний спускающиеся пути на ориентированной диаграмме отвечают ядрам элементарных сплетений $Q_{B C_{n}}^{B_{n}}$ и $Q_{B_{n-1}}^{B C_{n}}$ соответственно. Свертка ядер $Q_{B C_{n}}^{B_{n}}$ и $Q_{B_{n-1}}^{B C_{n}}$ в (1.109) при $\lambda_{n}=0$ отвечает интегрированию по переменным $z_{n, i}$, соответствующим внутренним вершинам поддиаграммы (1.120).

Подобно случаю $\mathfrak{g l}_{\ell+1}$, операторы рекурсии $Q_{B_{n-1}}^{B_{n}}$ можно рассматривать как специальные вырождения $\mathscr{Q}$-операторов Бакстера для аффинных $B_{\ell}^{(1)}$-цепочек Тоды. Ниже мы приводим интегральные представления для этих Q-операторов. Подчеркнем, что ранее $\mathscr{Q}$-операторы были известны только для случая $\widehat{\mathfrak{g l}}_{\ell+1}$. Мы не будем здесь приводить полный список свойств, характеризующих определяемые $\mathscr{Q}$-операторы, и ограничимся рассмотрением коммутационных соотношений для квадратичных гамильтонианов аффинных цепочек Тоды. Подробности будут опубликованы в другом месте. 
Начнем с описания $B_{\ell}^{(1)}$-цепочки Тоды. Множество простых корней аффинной системы корней $B_{\ell}^{(1)}$ можно записать в следующем виде:

$$
\alpha_{1}=\epsilon_{1}, \quad \alpha_{i+1}=\epsilon_{i+1}-\epsilon_{i}, \quad 1 \leqslant i \leqslant \ell-1, \quad \alpha_{\ell+1}=-\epsilon_{\ell}-\epsilon_{\ell-1} .
$$

Соответствующая диаграмма Дынкина имеет вид

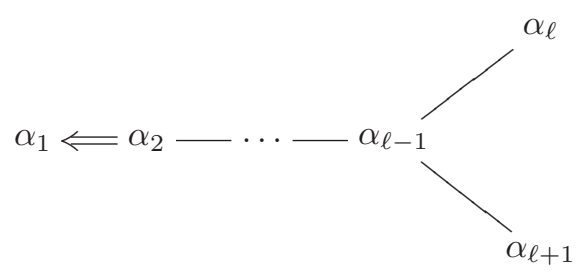

Эти корневые данные определяют аффинную $B_{\ell}^{(1)}$-цепочку Тоды с квадратичным гамильтонианом

$$
\mathscr{H}_{2}^{B_{\ell}^{(1)}}=-\frac{1}{2} \sum_{i=1}^{\ell+1} \frac{\partial^{2}}{\partial x_{i}^{2}}+\frac{1}{2} e^{x_{1}}+\sum_{i=1}^{\ell-1} e^{x_{i+1}-x_{i}}+g e^{-x_{\ell}-x_{\ell-1}}
$$

Здесь $g-$ произвольная константа связи.

Определим $\mathscr{Q}$-оператор Бакстера для $B_{\ell}^{(1)}$-цепочки Тоды как интегральный оператор с ядром

$$
\begin{aligned}
\mathscr{Q}^{B_{\ell}^{(1)}} & \left(\underline{x}^{(\ell)}, \underline{y}^{(\ell)} ; \lambda\right)=\int d \underline{z}^{(\ell)}\left(e^{x_{1}}+e^{y_{1}}\right)^{2 \imath \lambda}\left(e^{-x_{\ell}}+e^{-y_{\ell}}\right)^{-2 \imath \lambda} \\
& \times \exp \left\{-\imath \lambda\left(\sum_{i=1}^{\ell} x_{i}+2 z_{1}-2 \sum_{i=2}^{\ell} z_{i}+\sum_{i=1}^{\ell} y_{i}\right)\right\} \\
& \times Q_{B C_{\ell}^{(1)}}^{B_{\ell}^{(1)}}\left(\underline{x}^{(\ell)}, \underline{z}^{(\ell)}\right) Q_{B_{\ell}^{(1)}}^{B C_{\ell}^{(1)}}\left(\underline{z}^{(\ell)}, \underline{y}^{(\ell)}\right)
\end{aligned}
$$

где

$$
\begin{gathered}
Q_{B_{\ell}^{B C_{\ell}^{(1)}}}^{(1)}\left(\underline{z}^{(\ell)}, \underline{y}^{(\ell)}\right)=\exp \left\{-\left(\frac{1}{2} e^{z_{1}}+\sum_{i=1}^{\ell-1}\left(e^{y_{i}-z_{i}}+e^{z_{i+1}-y_{i}}\right)\right.\right. \\
\left.\left.+e^{y_{\ell}-z_{\ell}}+g e^{-y_{\ell}-z_{\ell}}\right)\right\}
\end{gathered}
$$

и

$$
Q_{B C_{n}^{(1)}}^{B_{n}^{(1)}}\left(\underline{x}^{(\ell)}, \underline{z}^{(\ell)}\right)=Q_{B_{n}^{(1)}}^{B C_{n}^{(1)}}\left(\underline{z}^{(\ell)}, \underline{x}^{(\ell)}\right)
$$

Здесь $\underline{x}^{(\ell)}=\left(x_{1}, \ldots, x_{\ell}\right), \underline{z}^{(\ell)}=\left(z_{1}, \ldots, z_{\ell}\right)$ и $\underline{y}^{(\ell)}=\left(y_{1}, \ldots, y_{\ell}\right)$.

Следующее предложение можно доказать непосредственной проверкой. 
ПреДЛОЖениЕ 1.7. Q-оператор (1.123) коммутирует с квадратичным гамильтонианом $B_{\ell}^{(1)}$-цепочки Тодъ, т.е. ядро сплетает гамильтонианы $\mathscr{H}_{2}^{B_{\ell}^{(1)}}$ :

$$
\mathscr{H}_{2}^{B_{\ell}^{(1)}}\left(\underline{x}^{(\ell)}\right) \mathscr{Q}^{B_{\ell}^{(1)}}\left(\underline{x}^{(\ell)}, \underline{y}^{(\ell)} ; \lambda\right)=\mathscr{Q}^{B_{\ell}^{(1)}}\left(\underline{x}^{(\ell)}, \underline{y}^{(\ell)} ; \lambda\right) \mathscr{H}_{2}^{B_{\ell}^{(1)}}\left(\underline{y}^{(\ell)}\right) .
$$

Покажем, что оператор рекурсии $Q_{B_{\ell-1}}^{B_{\ell}}$ можно рассматривать как вырождение $\mathscr{Q}$-операторов Бакстера для $B_{\ell}^{(1)}$. Произведем небольшую модификацию оператора рекурсии $Q_{B_{\ell-1} \oplus B_{1}}^{B_{\ell}}$ с ядром:

$$
Q_{B_{\ell-1} \oplus B_{1}}^{B_{\ell}}\left(\underline{x}^{(\ell)}, \underline{y}^{(\ell)} ; \lambda\right):=e^{\imath \lambda y_{\ell}} Q_{B_{\ell-1}}^{B_{\ell}}\left(\underline{x}^{(\ell)}, \underline{y}^{(\ell-1)} ; \lambda\right),
$$

где $\underline{y}^{(\ell-1)}=\left(y_{1}, \ldots, y_{\ell-1}\right)$. Оператор (1.127) сплетает гамильтонианы $\mathfrak{s o}_{2 \ell+1^{-}}$ и $\mathfrak{s o}_{2 \ell-1} \oplus \mathfrak{s o}_{2}$-цепочек Тоды. Тем самым, для квадратичных гамильтонианов имеем

$$
\begin{aligned}
\mathscr{H}_{2}^{B_{\ell}}\left(\underline{x}^{(\ell)}\right) Q_{B_{\ell-1} \oplus B_{1}}^{B_{\ell}}\left(\underline{x}^{(\ell)}, \underline{y}^{(\ell)} ; \lambda\right)=Q_{B_{\ell-1} \oplus B_{1}}^{B_{\ell}} & \left.\underline{x}^{(\ell)}, \underline{y}^{(\ell)} ; \lambda\right) \\
& \times\left(\mathscr{H}_{2}^{B_{\ell-1}}\left(\underline{y}^{(\ell-1)}\right)+\mathscr{H}_{2}^{B_{1}}\left(y_{\ell}\right)\right),
\end{aligned}
$$

где $\mathscr{H}_{2}^{B_{1}}\left(y_{\ell}\right)=-\frac{1}{2} \frac{\partial^{2}}{\partial y_{\ell}^{2}}$. Ясно, что проекция указанного выше соотношения на подпространство функций $F\left(\underline{y}^{(\ell)}\right)=\exp \left(\imath \lambda y_{\ell}\right) f\left(\underline{y}^{(\ell-1)}\right)$ определяет исходный оператор рекурсии, который удовлетворяет соотношению

$$
\mathscr{H}_{2}^{B_{\ell}}\left(\underline{x}^{(\ell)}\right) Q_{B_{\ell-1}}^{B_{\ell}}\left(\underline{x}^{(\ell)}, \underline{y}^{(\ell)} ; \lambda\right)=Q_{B_{\ell-1}}^{B_{\ell}}\left(\underline{x}^{(\ell)}, \underline{y}^{(\ell-1)} ; \lambda\right)\left(\mathscr{H}_{2}^{B_{\ell-1}}\left(\underline{y}^{(\ell-1)}\right)+\frac{1}{2} \lambda^{2}\right) .
$$

Рассмотрим однопараметрическое семейство ядер

$$
\begin{aligned}
& \mathscr{Q}^{B_{\ell}^{(1)}}\left(\underline{x}^{(\ell)}, \underline{y}^{(\ell)} ; \lambda ; \varepsilon\right)=\varepsilon^{\imath \lambda} e^{\imath \lambda y_{\ell}} \int d \underline{z}^{(\ell)}\left(e^{x_{1}}+e^{y_{1}}\right)^{2 \imath \lambda}\left(\varepsilon e^{y_{\ell}-x_{\ell}}+1\right)^{-2 \imath \lambda} \\
& \quad \times \exp \left\{-\imath \lambda\left(\sum_{i=1}^{\ell} x_{i}+2 z_{1}-2 \sum_{i=2}^{\ell} z_{i}+\sum_{i=1}^{\ell-1} y_{i}\right)\right\} Q_{B C_{\ell}^{(1)}}^{B_{\ell}^{(1)}}\left(x_{i}, z_{i}\right) Q_{B_{\ell}^{(1)}}^{B C_{\ell}^{(1)}}\left(z_{i}, y_{i} ; \varepsilon\right),
\end{aligned}
$$

где

$Q_{B_{\ell}^{(1)}}^{B C_{\ell}^{(1)}}\left(\underline{z}_{\ell}, \underline{y}_{\ell} ; \varepsilon\right)=\exp \left\{-\left(\frac{1}{2} e^{z_{1}}+\sum_{i=1}^{\ell-1}\left(e^{y_{i}-z_{i}}+e^{z_{i+1}-y_{i}}\right)+\varepsilon e^{y_{\ell}-z_{\ell}}+\varepsilon^{-1} g e^{-y_{\ell}-z_{\ell}}\right)\right\}$,

которые получены из ядра оператора $\mathscr{Q}^{B_{\ell}^{(1)}}$ заменой переменных $y_{\ell}=y_{\ell}+\ln \varepsilon$. Рассмотрим предельное поведение $(1.129),(1.130)$ при $\varepsilon \rightarrow 0, g \varepsilon^{-1} \rightarrow 0$. Тогда имеет место следующее соотношение, связывающее $\mathscr{Q}$-оператор для $B_{\ell}^{(1)}$-цепочки Тоды и (модифицированный) оператор рекурсии для $\mathfrak{s o}_{2 \ell+1}$-функции Уиттекера:

$$
Q_{B_{\ell-1} \oplus B_{1}}^{B_{\ell}}\left(\underline{x}^{(\ell)}, \underline{y}^{(\ell)} ; \lambda\right)=\lim _{\substack{\varepsilon \rightarrow 0, g \varepsilon^{-1} \rightarrow 0}} \varepsilon^{-\imath \lambda} \mathscr{Q}^{B_{\ell}^{(1)}}\left(\underline{x}^{(\ell)}, \underline{y}^{(\ell)} ; \lambda ; \varepsilon\right) .
$$


1.4. Интегральные представления собственных функций $\mathfrak{s p}_{2 \ell}$-цепочки Тоды. В этом разделе мы получим аналог интегрального представления Гивенталя функций Уиттекера для алгебр Ли $\mathfrak{s p}_{2 \ell}$. Как и в случае $\mathfrak{s o}_{2 \ell+1}$, мы начнем с интегрального представления $\mathfrak{s p}_{2 \ell}$-функций Уиттекера, использующего факторизационную параметризацию. Затем мы рассмотрим модификацию факторизационной параметризации, приводящую к интегральным представлениям типа Гивенталя для $\mathfrak{s p}_{2 \ell}$-функций Уиттекера.

Рассмотрим систему корней типа $C_{\ell}$, отвечающую алгебре Ли $\mathfrak{s p}_{2 \ell}$. Пусть $\left\{\epsilon_{1}, \ldots, \epsilon_{\ell}\right\}$ - ортонормированный базис в пространстве $\mathbb{R}^{\ell}$. Мы используем следующую реализацию простых корней, кокорней и фундаментальных весов векторами в $\mathbb{R}^{\ell}$ :

$$
\begin{array}{lll}
\alpha_{1}=2 \epsilon_{1}, & \alpha_{1}^{\vee}=\epsilon_{1}, & \omega_{1}=\epsilon_{1}+\ldots+\epsilon_{\ell} \\
\alpha_{2}=\epsilon_{2}-\epsilon_{1}, & \alpha_{2}^{\vee}=\epsilon_{2}-\epsilon_{1}, & \omega_{2}=\epsilon_{2}+\ldots+\epsilon_{\ell}, \\
\ldots & \ldots & \ldots \\
\alpha_{\ell}=\epsilon_{\ell}-\epsilon_{\ell-1}, & \alpha_{\ell}^{\vee}=\epsilon_{\ell}-\epsilon_{\ell-1}, & \omega_{\ell}=\epsilon_{\ell} .
\end{array}
$$

Матрица Картана $\left\|a_{i j}\right\|=\left\|\left\langle\alpha_{i}^{\vee}, \alpha_{j}\right\rangle\right\|$ становится симметрической $\left\|b_{i j}\right\|=\left\|d_{i} a_{i j}\right\|$ при $d_{1}=2, d_{i}=1, i=2, \ldots, \ell$. Свяжем с этими корневыми данными $\mathfrak{s p}_{2 \ell}$-цепочку Тоды с квадратичным гамильтонианом

$$
\mathscr{H}_{2}^{C_{\ell}}=-\frac{1}{2} \sum_{i=1}^{\ell} \frac{\partial^{2}}{\partial z_{i}^{2}}+2 e^{2 z_{1}}+\sum_{i=1}^{\ell-1} e^{z_{i+1}-z_{i}} .
$$

Можно дополнить (1.132) до полного набора из $\ell$ попарно коммутирующих операторов $\mathscr{H}_{k}^{C_{\ell}}$ для $C_{\ell}$-цепочки Тоды. Мы ищем интегральные представления общих собственных функций этого полного набора гамильтонианов. Задача о собственных функциях квадратичного гамильтониана может быть записана в следующем виде:

$$
\mathscr{H}_{2}^{C_{\ell}} \Psi_{\lambda_{1}, \ldots, \lambda_{\ell}}^{C_{\ell}}\left(z_{1}, \ldots, z_{\ell}\right)=\frac{1}{2} \sum_{i=1}^{\ell} \lambda_{i}^{2} \Psi_{\lambda_{1}, \ldots, \lambda_{\ell}}^{C_{\ell}}\left(z_{1}, \ldots, z_{\ell}\right) .
$$

1.4.1. $\mathfrak{s p}_{2 \ell}$-функция Уиттекера: факторизационная параметризация. Приведенное слово, отвечающее элементу максимальной длины $w_{0}$ в группе Вейля алгебры Ли $\mathfrak{s p}_{2 \ell}$, можно представить в рекурсивной форме:

$$
I_{\ell}=\left(i_{1}, i_{2}, i_{3}, \ldots, i_{m_{\ell}}\right):=(1,212,32123, \ldots,(\ell \ldots 212 \ldots \ell)), \quad m_{\ell}=\ell^{2},
$$

где индексы $i_{k} \in \Gamma=\{1, \ldots, \ell\}$ отвечают отражениям относительно простых корней $\alpha_{i_{k}}$. Пусть $N_{+} \subset G$ - максимальная унипотентная подгруппа в $G=$ $\operatorname{Sp}(2 \ell)$. C приведенным словом $I_{\ell}$ связана следующая рекурсивная параметризация типичного элемента $v^{C_{\ell}} \in N_{+}$:

$$
v^{C_{\ell}}=v^{C_{\ell-1}} \mathfrak{X}_{C_{\ell-1}}^{C_{\ell}},
$$

где

$$
\begin{aligned}
\mathfrak{X}_{C_{\ell-1}}^{C_{\ell}}= & X_{\ell}\left(y_{\ell, 1}\right) \cdots X_{k}\left(y_{k, 2(\ell+1-k)-1}\right) \cdots X_{2}\left(y_{2,2 \ell-3}\right) \\
& \times X_{1}\left(y_{1, \ell}\right) X_{2}\left(y_{2,2 \ell-2}\right) \cdots X_{k}\left(y_{k, 2(\ell+1-k)}\right) \cdots X_{\ell}\left(y_{\ell, 2}\right) .
\end{aligned}
$$


Здесь $X_{i}(y)=e^{y f_{i}}$ и $f_{i} \equiv f_{\alpha_{i}}$ - образующие, отвечающие простым корням. Подмножество $N_{+}^{(0)}$, допускающее представление $(1.134),(1.135)$, открыто в $N_{+}$. Действие алгебры Ли $\mathfrak{s p}_{2 \ell}$ на $N_{+}$, заданное в $(1.10)$, определяет действие на пространстве функций на $N_{+}^{(0)}$. Следующее предложение явно описывает это действие на пространстве $V_{\mu}$ эквивариантных функций на $N_{+}^{(0)}$.

ПредложениЕ 1.8. Следующие дифференииалъные операторы определяют реализачию представления $\pi_{\mu}$ алгебрь $\mathscr{U}\left(\mathfrak{s p}_{2 \ell}\right)$ на пространстве $V_{\mu}$ в терминах факторизационной параметризации на $N_{+}^{(0)}$ :

$$
\begin{aligned}
F_{1}= & \frac{\partial}{\partial y_{1, \ell}}+\sum_{n=1}^{\ell-1} \prod_{j=n}^{\ell-1}\left(\frac{y_{2,2 j}}{y_{2,2 j-1}}\right)^{2}\left\{\frac{\partial}{\partial y_{1, n}}-\frac{\partial}{\partial y_{1, n+1}}\right\} \\
& +\sum_{n=1}^{\ell-1} \frac{y_{2,2 n}}{y_{1, n+1}}\left(1+\frac{y_{2,2 n}}{y_{2,2 n-1}}\right) \prod_{j=n+1}^{\ell-1}\left(\frac{y_{2,2 j}}{y_{2,2 j-1}}\right)^{2}\left\{\frac{\partial}{\partial y_{2,2 n-1}}-\frac{\partial}{\partial y_{2,2 n}}\right\} \\
F_{k}= & \frac{\partial}{\partial y_{k, 2(\ell+1-k)}}+\sum_{n=1}^{\ell-k} \prod_{j=n+1}^{\ell-k} \frac{y_{k+1,2 j}}{y_{k+1,2 j-1}} \frac{y_{k, 2(j+1)-1}}{y_{k, 2(j+1)}}\left\{\frac{\partial}{\partial y_{k, 2 n}}-\frac{\partial}{\partial y_{k, 2 n+1}}\right\} \\
& +\sum_{n=1}^{\ell-k} \frac{y_{k+1,2 n}}{y_{k, 2(n-1)}} \prod_{j=n}^{\ell-k} \frac{y_{k+1,2 j}}{y_{k+1,2 j-1}} \frac{y_{k, 2(j+1)-1}}{y_{k, 2(j+1)}}\left\{\frac{\partial}{\partial y_{k+1,2 n-1}}-\frac{\partial}{\partial y_{k+1,2 n}}\right\}
\end{aligned}
$$

$n p u k=2, \ldots, \ell u$

$$
\begin{aligned}
H_{k}= & \left\langle\mu, \alpha_{k}^{\vee}\right\rangle+\sum_{i=1}^{\ell} a_{k, i} \sum_{j=1}^{n_{i}} y_{i, j} \frac{\partial}{\partial y_{i, j}}, \quad k=1, \ldots, \ell \\
E_{1}= & \sum_{n=1}^{\ell} y_{1, n}\left\{-\left\langle\mu, \alpha_{1}^{\vee}\right\rangle+\sum_{j=1}^{2 n-3} y_{2, j} \frac{\partial}{\partial y_{2, j}}-2 \sum_{j=1}^{n-1} y_{1, j} \frac{\partial}{\partial y_{1, j}}-y_{1, n} \frac{\partial}{\partial y_{1, n}}\right\}, \\
E_{2}= & \sum_{n=1}^{2(\ell-1)} y_{2, n}\left\{-\left\langle\mu, \alpha_{2}^{\vee}\right\rangle+2 \sum_{j=1}^{\alpha(n)+1} y_{1, j} \frac{\partial}{\partial y_{1, j}}-2 \sum_{j=1}^{n-1} y_{2, j} \frac{\partial}{\partial y_{2, j}}\right. \\
& \left.+\sum_{j=1}^{2 \beta(n)-3} y_{3, j} \frac{\partial}{\partial y_{3, j}}-y_{2, n} \frac{\partial}{\partial y_{2, n}}\right\}, \\
E_{k}= & \sum_{n=1}^{2(\ell+1-k)} y_{k, n}\left\{-\left\langle\mu, \alpha_{k}^{\vee}\right\rangle+2 \sum_{j=1}^{2 \alpha(n)+1} y_{k-1, j} \frac{\partial}{\partial y_{k-1, j}}-2 \sum_{j=1}^{n-1} y_{k, j} \frac{\partial}{\partial y_{k, j}}\right. \\
& \left.+\sum_{j=1}^{2 \beta(n)-3} y_{k+1, j} \frac{\partial}{\partial y_{k+1, j}}-y_{k, n} \frac{\partial}{\partial y_{k, n}}\right\}, \quad k=2, \ldots, \ell
\end{aligned}
$$

где $E_{i}=\pi_{\mu}\left(e_{i}\right), H_{i}=\pi_{\mu}\left(h_{i}\right), F_{i}=\pi_{\mu}\left(f_{i}\right), i=1, \ldots, \ell, n_{1}=\ell, n_{k}=2(\ell+1-k)$ при $1<k \leqslant \ell$ u $\alpha(n):=[n / 2], \beta(n):=[(n+1) / 2]$.

Доказательство дано в п. 2.4.3 части 2. 
Левый и правый векторы Уиттекера определены в (1.7), (1.8) и имеют следующее выражение в факторизационной параметризации.

Лемма 1.7. Левъй и правый векторы Уиттекера в факторизационной параметризачии имеют вид

$$
\begin{aligned}
\psi_{R}(y)= & \exp \left\{-\left(\sum_{n=1}^{\ell} y_{1, n}+\sum_{k=2}^{\ell} \sum_{n=1}^{n_{k}} y_{k, n}\right)\right\} \\
\psi_{L}(y)=\prod_{i=1}^{\ell} & \left(\prod_{n=1}^{\ell} y_{1, n} \prod_{k=2}^{i} \prod_{n=1}^{2(\ell+1-k)} y_{k, n} \prod_{k=i+1}^{\ell} \prod_{n=1}^{\ell+1-k} y_{k, 2 n-1}^{2}\right)^{\left\langle\mu, \alpha_{i}^{\vee}\right\rangle} \\
& \times \exp \left\{-\left(\sum_{n=1}^{\ell} \frac{1}{y_{1, n}}\left(1+\frac{y_{2,2(n-1)}}{y_{2,2(n-1)-1}}\right)^{2} \prod_{i=n+1}^{\ell}\left(\frac{y_{2,2(i-1)}}{y_{2,2(i-1)-1}}\right)^{2}\right.\right. \\
& \left.\left.+\sum_{k=2}^{\ell} \sum_{n=1}^{n_{k} / 2} \frac{1}{y_{k, 2 n}}\left(1+\frac{y_{k+1,2(n-1)}}{y_{k+1,2(n-1)-1}}\right) \prod_{i=n+1}^{n_{k} / 2} \frac{y_{k+1,2(i-1)}}{y_{k+1,2(i-1)-1}} \frac{y_{k, 2 i-1}}{y_{k, 2 i}}\right)\right\}
\end{aligned}
$$

где $n_{1}=\ell$ u $n_{k}=2(\ell+1-k)$ nрu $k=2, \ldots, \ell$.

Доказательство дано в п. 2.3.3 части 2.

Используя выражения (1.140) для левого и правого векторов, мы получаем интегральное представление для $\mathfrak{s p}_{2 \ell}$-функции Уиттекера в терминах факторизационной параметризации.

ТЕОРема 1.8. Собственные функции $\mathfrak{s p}_{2 \ell}$-цепочки Тоды допускают интегральное представление:

$$
\begin{aligned}
\Psi_{\lambda_{1}, \ldots, \lambda_{\ell}}^{C_{\ell}}\left(z_{1}, \ldots, z_{\ell}\right)=e^{\imath \lambda_{1} z_{1}+\cdots+\imath \lambda_{\ell} z_{\ell}} \int_{C} \prod_{i=1}^{\ell} \prod_{k=1}^{n_{i}} \frac{d y_{i, k}}{y_{i, k}}\left(\prod_{n=1}^{\ell} y_{1, n} \prod_{k=2}^{\ell} \prod_{n=1}^{n_{k} / 2} y_{k, 2 n-1}^{2}\right)^{\imath \lambda_{1}} \\
\quad \times \prod_{i=2}^{\ell}\left(\prod_{n=1}^{\ell} y_{1, n} \prod_{k=2}^{i} \prod_{n=1}^{n_{k}} y_{k, n} \prod_{k=i+1}^{\ell} \prod_{n=1}^{n_{k} / 2} y_{k, 2 n-1}^{2}\right)^{\imath\left(\lambda_{i}-\lambda_{i-1}\right)} \\
\quad \times \exp \left\{-\left(\sum_{n=1}^{\ell} \frac{1}{y_{1, n}}\left(1+\frac{y_{2,2(n-1)}}{y_{2,2(n-1)-1}}\right)^{2} \prod_{i=n+1}^{\ell}\left(\frac{y_{2,2(i-1)}}{y_{2,2(i-1)-1}}\right)^{2}\right.\right. \\
\quad+\sum_{k=2}^{\ell} \sum_{n=1}^{n_{k} / 2} \frac{1}{y_{k, 2 n}}\left(1+\frac{y_{k+1,2(n-1)}}{y_{k+1,2(n-1)-1}}\right) \prod_{i=n+1}^{n_{k} / 2} \frac{y_{k+1,2(i-1)}}{y_{k+1,2(i-1)-1}} \frac{y_{k, 2 i-1}}{y_{k, 2 i}} \\
\left.\left.+e^{2 z_{1}} \sum_{n=1}^{\ell} y_{1, n}+\sum_{k=2}^{\ell} e^{z_{k}-z_{k-1}} \sum_{n=1}^{n_{k}} y_{k, n}\right)\right\},
\end{aligned}
$$

где $n_{1}=\ell$ u $n_{k}=2(\ell+1-k)$ при $k=2, \ldots, \ell$. Область интегрирования $C \subset N_{+}$ является некомпактным подмногообразием половинной размерности, для которого подынтегральная функция экспоненииально убывает на гранище и на бесконечности. В частности, в качестве $C$ можсно взять малую деформацию подмножества $\mathbb{R}_{+}^{\ell^{2}}$ в $\mathbb{C}^{\ell^{2}}$, обеспечивающую сходимость интеграла. 
Доказательство дано в п. 2.3.3 части 2.

ПримеР 1.4. При $\ell=2$ общая формула (1.141) принимает вид

$$
\begin{aligned}
\Psi_{\lambda_{1}, \lambda_{2}}^{C_{2}}\left(z_{1}, z_{2}\right)= & e^{\imath \lambda_{1} z_{1}+\imath \lambda_{2} z_{2}} \int_{C} \prod_{i, k=1}^{2} \frac{d y_{i, k}}{y_{i, k}}\left(y_{1,1} y_{2,1}^{2} y_{1,2}\right)^{\imath \lambda_{1}}\left(y_{2,1} y_{1,2} y_{2,2}\right)^{\imath \lambda_{2}-\imath \lambda_{1}} \\
& \times \exp \left\{-\left(\frac{1}{y_{1,1}}\left(\frac{y_{2,2}}{y_{2,1}}\right)^{2}+\frac{1}{y_{1,2}}\left(\frac{y_{2,2}}{y_{2,1}}+1\right)^{2}+\frac{1}{y_{2,2}}\right.\right. \\
& \left.+e^{2 z_{1}}\left(y_{1,1}+y_{1,2}\right)+e^{z_{2}-z_{1}}\left(y_{2,1}+y_{2,2}\right)\right\}
\end{aligned}
$$

1.4.2. $\mathfrak{s p}_{2 \ell}$-функция Уиттекера: модифицированная факторизационная параметризация. В этом пункте мы определяем модифицированную факторизационную параметризацию на открытом подмножестве в $N_{+} \subset \operatorname{Sp}(2 \ell)$. Мы используем эту параметризацию для построения интегральных представлений $\mathfrak{s p}_{2 \ell}$-функций Уиттекера. По аналогии с рассмотренными выше интегральными представлениями $\mathfrak{s o}_{2 \ell+1}$-функций Уиттекера эти представления имеют простую рекурсивную структуру относительно ранга $\ell$. Они могут быть описаны в сугубо комбинаторных терминах с использованием подходящих графов. Эти представления можно рассматривать как обобщение интегральных представлений Гивенталя на случай $\mathfrak{g}=\mathfrak{s p}_{2 \ell}$.

Мы следуем тому же подходу, который был использован в описании модифицированного факторизационного представления в случае $\mathfrak{s o}_{2 \ell+1}$. Имеется реализация тавтологического представления $\pi: \mathfrak{s p}_{2 \ell} \rightarrow \operatorname{End}\left(\mathbb{C}^{2 \ell}\right)$, для которого образующие Шевалле, отвечающие борелевской (соответственно картановской) подалгебре в $\mathfrak{s p}_{2 \ell}$, переходят в верхнетреугольные (соответственно диагональные) матрицы. Это определяет вложение $\mathfrak{s p}_{2 \ell} \subset \mathfrak{g l}_{2 \ell}$, при котором борелевская (соответственно картановская) подалгебра отображается в борелевскую (соответственно картановскую) подалгебру (см., например, [17]). Для того чтобы определить соответствующее вложение групп, рассмотрим следующую инволюцию на $\mathrm{GL}(2 \ell)$ :

$$
g \longmapsto g^{*}:=\dot{W}_{0}^{-1}\left(g^{-1}\right)^{t} \dot{W}_{0},
$$

где $a \rightarrow a^{t}$ индуцировано стандартным транспонированием матриц и $\dot{W}_{0}-$ это подъем элемента максимальной длины группы Вейля $S_{2 \ell}$ для $\mathfrak{g l}_{2 \ell}$. В матричной форме это может быть записано как

$$
\pi\left(\dot{W}_{0}\right)=S J
$$

где $S=\operatorname{diag}(1,-1, \ldots, 1,-1)$ и $J=\left\|J_{i, j}\right\|=\left\|\delta_{i+j, 2 \ell+1}\right\|$. Тогда симплектическая группа $G=\operatorname{Sp}(2 \ell)$ отождествляется со следующей подгруппой в $\mathrm{GL}(2 \ell)$ (см., например, [17]):

$$
\operatorname{Sp}(2 \ell)=\left\{g \in \mathrm{GL}(2 \ell): g^{*}=g\right\} .
$$

Пусть $\epsilon_{i, j}$ - элементарная $(2 \ell \times 2 \ell)$-матрица с единицей на месте $(i, j)$ и нулями на прочих местах. Рассмотрим следующие $(2 \ell \times 2 \ell)$-матрицы:

$$
U_{n}=\sum_{i=1}^{\ell} \epsilon_{i, i}+\sum_{i=1}^{n} e^{-x_{n, i}} \epsilon_{\ell+i, \ell+i}+\sum_{i=n+1}^{\ell} \epsilon_{\ell+i, \ell+i}
$$




$$
\begin{aligned}
& \widetilde{U}_{n}=U_{n}+e^{z_{n, 1}} \epsilon_{\ell, \ell+1}+\sum_{i=1}^{n-1} e^{-z_{n-1, i}} \epsilon_{\ell+i, \ell+1+i}, \\
& \widetilde{U}_{n}^{\prime}=U_{n}+\sum_{i=1}^{n-1} e^{-z_{n-1, i}} \epsilon_{\ell+i, \ell+1+i} \\
& V_{n}=\sum_{i=1}^{\ell-n} \epsilon_{i, i}+e^{-z_{n, 1}} \epsilon_{\ell+1, \ell+1}+\sum_{i=1}^{n} e^{z_{n, i}} \epsilon_{\ell+1-i, \ell+1-i}, \\
& \widetilde{V}_{n}=V_{n}+e^{x_{n, 1}} \epsilon_{\ell, \ell+1}+\sum_{i=1}^{n} e^{x_{n, i}} \epsilon_{\ell+1-i, \ell+2-i}, \\
& \widetilde{V}_{n}^{\prime}=V_{n}+\sum_{i=1}^{n} e^{x_{n, i}} \epsilon_{\ell+1-i, \ell+2-i} .
\end{aligned}
$$

Модифицированная факторизационная параметризация может быть определена следующим образом.

Tеорема 1.9. (i) Образ типичного элемента $v^{C_{\ell}} \in N_{+}^{(0)}$ при тавтологическом представлении $\pi: \mathfrak{s p}_{2 \ell} \rightarrow \operatorname{End}\left(\mathbb{C}^{2 \ell}\right)$ может быть представлен в виде

$$
\pi\left(v^{C_{\ell}}\right)=\mathfrak{X}_{1} \mathfrak{X}_{2} \ldots \mathfrak{X}_{\ell}, \quad \mathfrak{X}_{n}=\pi\left(\mathfrak{X}_{C_{n-1}}^{C_{n}}\right), \quad n=1, \ldots, \ell,
$$

zдe

$$
\begin{gathered}
\mathfrak{X}_{1}=1+e^{x_{1,1}+z_{1,1}} \epsilon_{\ell-1, \ell}, \\
\mathfrak{X}_{n}=\left[\widetilde{U}_{n}^{\prime} U_{n}^{-1}\right]^{*} \widetilde{U}_{n} U_{n}^{-1} \cdot \widetilde{V}_{n} V_{n}^{-1}\left[\widetilde{V}_{n}^{\prime} V_{n}^{-1}\right]^{*}, \quad n=2, \ldots, \ell,
\end{gathered}
$$

$u z_{\ell, k}=0, k=1, \ldots, \ell$.

(ii) Формуль $(1.150),(1.151)$ определяют параметризацию множества полностъю неотрицателъных унипотентных элементов $N_{+}^{(0)} \subset N_{+}$.

ДокАзАТЕЛьство. Пусть $v^{C_{\ell}}(y)$ - параметризация на открытом подмножестве в $N_{+}$из $(1.134),(1.135)$. Рассмотрим унипотентные элементы $\widetilde{X}_{k}(y)=$ $e^{y \epsilon_{\ell+1-k, \ell+2-k}}, k=1, \ldots, \ell$, в $\mathrm{GL}(2 \ell)$ и действие инволюции * , заданное правилом:

$$
\widetilde{X}_{1}(y)^{*}=\widetilde{X}_{1}(y), \quad \tilde{X}_{i}(y)^{*}=\tilde{X}_{\ell+i}(y), \quad i=2, \ldots, \ell .
$$

Вложим элементарные унипотентные подгруппы $X_{i}(y)$ из $\operatorname{Sp}(2 \ell)$ в $\mathrm{GL}(2 \ell)$ следующим образом:

$$
X_{1}(y)=\widetilde{X}_{1}(y), \quad X_{i}(y)=\widetilde{X}_{i}(y)^{*} \widetilde{X}_{i}(y), \quad i=2, \ldots, \ell .
$$

Тем самым, произвольный регулярный унипотентный элемент $v^{C_{\ell}}$ вложен в

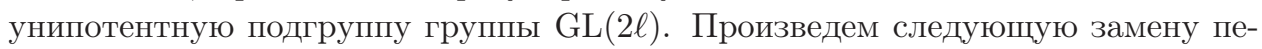
ременных:

$$
\begin{aligned}
y_{1,1} & =e^{x_{1,1}+z_{1,1}}, & & \\
y_{1, k} & =e^{z_{k-1,1}+x_{k, 1}}+e^{z_{k, 1}+x_{k, 1}}, & & k=2, \ldots, \ell, \\
y_{k, 2 r-1} & =e^{x_{k+r-1, k}-z_{k+r-2, k-1}}, & & r=1, \ldots, \ell+1-k, \\
y_{k, 2 r} & =e^{x_{k+r-1, k}-z_{k+r-1, k-1}} . & &
\end{aligned}
$$

Здесь $z_{\ell, k}=0$ при $k=1, \ldots, \ell$. 
Несложно проверить, что после замены переменных (1.152) образ $\pi\left(v^{C_{\ell}}\right)$ элемента $v^{C_{\ell}}$, определенный в (1.134), (1.135), преобразуется в (1.150), (1.151). Учитывая, что замена переменных обратима (1.152), мы получаем параметризацию на $N_{+}^{(0)} \subset N_{+}$. Теорема 1.9 доказана.

Модифицированная факторизационная параметризация на унипотентной группе $N_{+}$определяет специальную реализацию представления основной серии алгебры $\mathscr{U}\left(\mathfrak{s p}_{2 \ell}\right)$ дифференциальными операторами. Ее можно получить, используя замену переменных (1.152) в реализации из предложения 1.8. Мы будем называть эту реализацию представления алгебры $\mathscr{U}\left(\mathfrak{s p}_{2 \ell}\right)$ представлением Гаусса-Гивенталя.

ПредложениЕ 1.9. Следующие дифференциалъные операторы определяют представление $\pi_{\mu}$ алгебры $\mathscr{U}\left(\mathfrak{s p}_{2 \ell}\right)$ в пространстве $V_{\mu}$ в терминах модифицированной факторизационной параметризащии:

$$
\begin{aligned}
& F_{1}=\sum_{n=1}^{2 \ell-1} e^{z_{\beta(n), 1}-x_{\alpha(n)+1,1}}\left\{\frac{\partial}{\partial x_{1,1}}+\sum_{k=2}^{\beta(n)}\left(\frac{\partial}{\partial x_{k, 1}}-\frac{\partial}{\partial x_{k, 2}}\right)-\sum_{k=1}^{\alpha(n)} \frac{\partial}{\partial z_{k, 1}}\right\}, \\
& F_{2}=-\sum_{n=1}^{2 \ell-3} e^{z_{\beta(n)+1,2}-x_{\alpha(n)+2,2}}\left\{\frac{\partial}{\partial x_{1,1}}-\frac{\partial}{\partial z_{1,1}}\right. \\
& +\sum_{k=1}^{\beta(n)}\left(\frac{\partial}{\partial x_{k+1,1}}-\frac{\partial}{\partial x_{k+1,2}}+\left(1-\delta_{n, 1}\right) \frac{\partial}{\partial x_{k+1,3}}\right) \\
& \left.-\sum_{k=1}^{\alpha(n)}\left(\frac{\partial}{\partial z_{k+1,1}}-\frac{\partial}{\partial z_{k+1,2}}\right)+(-1)^{n} \frac{e^{z_{\beta(n)+1,1}}}{e^{x_{\alpha(n)+1,1}}+e^{x_{\alpha(n)+2,1}}} \frac{\partial}{\partial x_{\alpha(n)+2,1}}\right\} \\
& F_{k}=\sum_{2(\ell-k)+1} e^{z_{\beta(n)+k-1, k}-x_{\alpha(n)+k, k}}\left\{\frac{\partial}{\partial z_{k-1, k-1}}+\frac{\partial}{\partial x_{k, k}}\right. \\
& +\sum_{m=1}^{\alpha(n)}\left(\frac{\partial}{\partial z_{k-1+m, k-1}}-\frac{\partial}{\partial z_{k-1+m, k}}\right) \\
& \left.+\sum_{m=1}^{\beta(n)}\left(\frac{\partial}{\partial x_{k-1+m, k}}-\frac{\partial}{\partial x_{k-1+m, k+1}}\right)\right\}, \quad k=3, \ldots, \ell, \\
& H_{k}=\left\langle\mu, \alpha_{k}^{\vee}\right\rangle+\sum_{n=1}^{\ell} a_{k, n} \sum_{i=n}^{\ell} \frac{\partial}{\partial x_{i, n}}, \quad k=1, \ldots, \ell, \\
& E_{1}=-\sum_{n=1}^{2 \ell-1} e^{z_{\beta(n), 1}+x_{\alpha(n)+1,1}}\left\{\mu_{1}+\sum_{k=1}^{\beta(n)} \frac{\partial}{\partial x_{k, 1}}+\sum_{k=1}^{\alpha(n)} \frac{\partial}{\partial z_{k, 1}}\right\}, \\
& E_{2}=\sum_{n=1}^{2 \ell-2} e^{x_{\beta(n)+1,2}-z_{\alpha(n)+1,1}}\left\{\mu_{1}-\mu_{2}+\frac{\partial}{\partial x_{1,1}}+\frac{\partial}{\partial z_{1,1}}\right. \\
& \left.+\sum_{k=1}^{\alpha(n)}\left(\frac{\partial}{\partial x_{k+1,1}}-\frac{\partial}{\partial x_{k+1,2}}\right)+\sum_{k=1}^{\beta(n)-1}\left(\frac{\partial}{\partial z_{k+1,1}}-\frac{\partial}{\partial z_{k+1,2}}\right)\right\}
\end{aligned}
$$




$$
\begin{aligned}
E_{k}= & \sum_{n=1}^{2(\ell+1-k)} e^{x_{\beta(n)+k-1, k}-z_{\beta(n)+k-1, k-1}}\left\{\left\langle\mu, \alpha_{k}^{\vee}\right\rangle+\frac{\partial}{\partial x_{k-1, k-1}}+\frac{\partial}{\partial z_{k-1, k-1}}\right. \\
& +\sum_{m=1}^{\alpha(n)}\left(\frac{\partial}{\partial x_{k-1+m, k-1}}-\frac{\partial}{\partial x_{k-1+m, k}}\right) \\
& \left.+\sum_{m=2}^{\beta(n)}\left(\frac{\partial}{\partial z_{k-2+m, k-1}}-\frac{\partial}{\partial z_{k-2+m, k}}\right)\right\}, \quad k=3, \ldots, \ell .
\end{aligned}
$$

Здесъ $z_{\ell, i}=0, i=1, \ldots, \ell$, и дифференцирования над $x_{i, k}, z_{i, k}, i<k$, опущень. Мъ используем обозначения $E_{i}=\pi_{\mu}\left(e_{i}\right), F_{i}=\pi_{\mu}\left(f_{i}\right), H_{i}=\pi_{\mu}\left(h_{i}\right), i=1, \ldots, \ell$, $u \alpha(n):=[n / 2], \beta(n):=[(n+1) / 2]$.

Сейчас мы получим явное выражение матричного элемента (1.12), использующее определенное выше представление Гаусса-Гивенталя. Векторы Уиттекера $\psi_{R}$ и $\psi_{L}$ в этом представлении удовлетворяют системе дифференциальных уравнений (1.7) и (1.8). Найдем общий вид их решений. Используя явную замену переменных (1.152), мы получаем выражения для векторов Уиттекера в модифицированной факторизационной параметризации.

ЛЕмма 1.8. Имеют место следующие выражения для левого и правого векторов Уиттекера:

$$
\begin{aligned}
\psi_{R}= & \exp \left\{-e^{x_{1,1}+z_{1,1}}-\sum_{n=2}^{\ell}\left(e^{z_{n-1,1}+x_{n, 1}}+e^{z_{n, 1}+x_{n, 1}}\right)\right. \\
& \left.-\sum_{k=2}^{\ell} \sum_{n=k}^{\ell}\left(e^{x_{n, k}-z_{n-1, k}-1}+e^{x_{n, k}-z_{n, k}-1}\right)\right\}, \\
\psi_{L}=e^{\mu_{1} z_{1,1}} \prod_{n=2}^{\ell}\left(e^{z_{n, 1}}+e^{z_{n-1,1}}\right)^{\mu_{n}} & \\
& \times \prod_{n=1}^{\ell} \exp \left\{-\mu_{n}\left(\sum_{i=1}^{n} z_{n, i}-x_{n, 1}-2 \sum_{i=2}^{n} x_{n, i}+\sum_{i=1}^{n-1} z_{n-1, i}\right)\right\} \\
& \times \exp \left\{-\sum_{k=1}^{\ell}\left(e^{z_{k, k}-x_{k, k}}+\sum_{n=k+1}^{\ell} e^{z_{n-1, k}-x_{n, k}}+e^{z_{n, k}-x_{n, k}}\right)\right\},
\end{aligned}
$$

где $z_{\ell, k}=0$ u $\mu_{k}=\imath \lambda_{k}-\rho_{k}, \rho_{k}=k$ npu $k=1, \ldots, \ell$.

Мы готовы указать интегральное представление для спаривания (1.12) в случае $\mathfrak{g}=\mathfrak{s p}_{2 \ell}$. Чтобы получить явное выражение для подынтегральной функции, мы используем разложение элемента максимального тора, как и для алгебр $\mathfrak{g l}_{\ell+1}$ и $\mathfrak{s o}_{2 \ell+1}$, рассмотренных в предыдущих разделах:

$$
e^{-H_{z}}=\pi_{\lambda}\left(\exp \left(-\sum_{i=1}^{\ell}\left\langle\omega_{i}, z\right\rangle h_{i}\right)\right)=e^{H_{L}} e^{H_{R}},
$$


где

$$
-H_{z}=H_{L}+H_{R}=-\left\langle\mu, z_{\ell}\right\rangle-2 z_{\ell, 1} \sum_{n=1}^{\ell} \frac{\partial}{\partial x_{n, 1}}+\sum_{k=1}^{\ell-1}\left(z_{\ell, i}-z_{\ell, i+1}\right) \sum_{n=k}^{\ell} \frac{\partial}{\partial x_{n, k}}
$$

И

$$
\begin{aligned}
H_{L} & =\sum_{k=1}^{\ell} z_{\ell, k}\left(\sum_{n=k}^{\ell-1} \frac{\partial}{\partial z_{n, k}}+\sum_{n=k}^{\ell} \frac{\partial}{\partial x_{n, k}}\right), \\
H_{R} & =-\left\langle\mu, z_{\ell}\right\rangle-\sum_{k=1}^{\ell-1} z_{\ell, k}\left(\sum_{n=k}^{\ell} \frac{\partial}{\partial x_{n, k}}-\sum_{n=k}^{\ell-1} \frac{\partial}{\partial z_{n, k}}-\sum_{n=k+1}^{\ell} \frac{\partial}{\partial x_{n, k+1}}\right) .
\end{aligned}
$$

Мы считаем, что $H_{L}$ действует на левом векторе и $H_{R}$ действует на правом векторе в (1.12). С учетом леммы 1.8 мы приходим к следующей теореме.

Теорема 1.10. Собственные функиии $\mathfrak{s p}_{2 \ell}$-цепочки Тоды (1.12) допускают интегральное представление

$$
\Psi_{\lambda_{1}, \ldots, \lambda_{\ell}}^{C_{\ell}}\left(z_{1}, \ldots, z_{\ell}\right)=\int_{C} \prod_{k=1}^{\ell-1} d \underline{z}_{k} \prod_{k=1}^{\ell} d \underline{x}_{k} e^{\mathscr{F}^{C_{\ell}}}
$$

где

$$
\begin{aligned}
\mathscr{F}^{C_{\ell}=\imath} \lambda_{1} z_{1,1}-\sum_{n=2}^{\ell} \imath \lambda_{n}\left(\sum_{i=1}^{n} z_{n, i}-x_{n, 1}-2 \sum_{i=2}^{n} x_{n, i}\right. \\
\left.+\sum_{i=1}^{n-1} z_{n-1, i}-\ln \left(e^{z_{n, 1}}+e^{z_{n-1,1}}\right)\right) \\
-\left\{\sum_{k=1}^{\ell}\left(e^{z_{k, k}-x_{k, k}}+\sum_{n=k+1}^{\ell} e^{z_{n-1, k}-x_{n, k}}+e^{z_{n, k}-x_{n, k}}\right)+e^{x_{1,1}+z_{1,1}}\right. \\
\left.+\sum_{n=2}^{\ell}\left(e^{z_{n-1,1}+x_{n, 1}}+e^{z_{n, 1}+x_{n, 1}}\right)+\sum_{k=2}^{\ell} \sum_{n=k}^{\ell}\left(e^{x_{n, k}-z_{n-1, k-1}}+e^{x_{n, k}-z_{n, k-1}}\right)\right\}
\end{aligned}
$$

и мы полагаем $z_{i}:=z_{\ell, i}, 1 \leqslant i \leqslant \ell$. Здесъ $C \subset N_{+}-$некомпактное подмногообразие половинной размерности, для которого подынтегралъные выражения экспоненциально убывают на гранище и на бесконечности. В частности, в качестве области интегрирования можно взять малую деформацию подпространства $\mathbb{R}^{\ell^{2}} \subset \mathbb{C}^{\ell^{2}}$, обеспечивающую сходимость интеграла.

Заметим, что интеграл $(1.165),(1.166)$ можно получить из (1.141) следующей подстановкой:

$$
\begin{aligned}
& y_{1,1}=e^{-2 z_{\ell, 1}} e^{x_{1,1}+z_{1,1}}, \\
& y_{1, k}=e^{-2 z_{\ell, 1}}\left(e^{z_{k-1,1}+x_{k, 1}}+e^{z_{k, 1}+x_{k, 1}}\right), \quad k=2, \ldots, \ell, \\
& y_{k, 2 r-1}=e^{-2 z_{\ell, k-1}-z_{\ell, k}} e^{x_{k+r-1, k}-z_{k+r-2, k-1}}, \quad r=1, \ldots, \ell+1-k, \\
& y_{k, 2 r}=e^{-2 z_{\ell, k-1}-z_{\ell, k}} e^{x_{k+r-1, k}-z_{k+r-1, k-1}} .
\end{aligned}
$$


ПримеР 1.5. При $\ell=2$ общая формула (1.165) принимает вид

$$
\begin{aligned}
\Psi_{\lambda_{1}, \lambda_{2}}^{C_{2}} & \left(z_{1}, z_{2}\right)=\int_{C} d x_{1,1} d x_{2,1} d x_{2,2} d z_{1,1}\left(e^{z_{1,1}}+e^{z_{2,1}}\right)^{\imath \lambda_{2}} \\
& \times \exp \left\{\imath \lambda_{1} x_{1,1}-\imath \lambda_{2}\left(z_{2,1}+z_{2,2}-\left(x_{2,1}+2 x_{2,2}\right)+z_{1,1}\right)\right. \\
& -\left(e^{z_{1,1}-x_{1,1}}+e^{x_{1,1}+z_{1,1}}+e^{z_{1,1}-x_{2,1}}+e^{z_{1,1}+x_{2,1}}+e^{z_{2,2}-x_{2,2}}\right. \\
& \left.\left.+e^{z_{2,1}-x_{2,1}}+e^{z_{1,1}+x_{2,1}}+e^{x_{2,2}-z_{1,1}}+e^{x_{2,2}-z_{2,1}}\right)\right\}
\end{aligned}
$$

где $z_{1}=z_{2,1}, z_{2}=z_{2,2}$.

Имеется простое комбинаторное описание потенциала $\mathscr{F}^{C_{\ell}}$ на нулевом уровне спектра $\left\{\lambda_{i}=0\right\}$. А именно, он может быть представлен как сумма по всем стрелкам на следующей диаграмме:

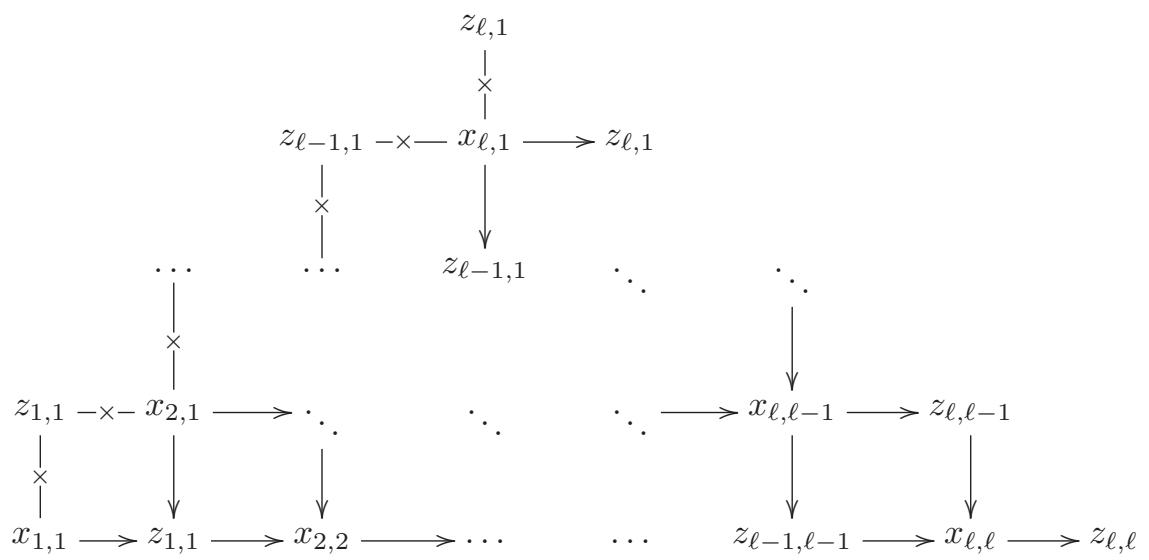

Мы используем то же правило сопоставления переменных стрелкам диаграммы, что и для случая $A_{\ell}$. В дополнение мы сопоставляем символу $z-\times-x$ экспоненту $e^{z+x}$.

Заметим, что диаграмма $C_{\ell}$ может быть получена факторизацией диаграммы для $A_{2 \ell-1}$. Рассмотрим инволюцию

$$
*: X \longmapsto-\dot{W}_{0}^{-1} X^{t} \dot{W}_{0},
$$

где $\dot{W}_{0}$ - подъем элемента максимальной длины группы Вейля $S_{2 \ell}$ и $X^{t}-$ стандартное транспонирование. Соответствующее действие на модифицированных параметрах факторизации имеет вид

$$
*: x_{k, i} \longmapsto-x_{k, k+1-i} .
$$

Это определяет факторизацию $A_{2 \ell-1}$-диаграммы, которая приводит к диаграмме для $C_{\ell}$.

Несложно выписать $C_{\ell}$-аналог соотношений (1.52). Рассмотрим переменные

$$
\begin{gathered}
a_{n, 1}=e^{x_{n, 1}+z_{n-1,1}}, \quad a_{n, i}=e^{x_{n, i}-z_{n-1, i-1}}, \quad 2 \leqslant i \leqslant n \leqslant \ell ; \\
b_{k, 1}=e^{x_{k, 1}+z_{k, 1}}, \quad k=1, \ldots, \ell ; \quad b_{n, i}=e^{z_{n, i}-x_{n, i-1}}, \quad 2 \leqslant i \leqslant n \leqslant \ell ; \\
c_{k, i}=e^{z_{k, i}-x_{k, i}}, \quad 1 \leqslant i \leqslant k \leqslant \ell ; \\
d_{m, j}=e^{z_{m, j}-x_{m+1, j}}, \quad 1 \leqslant j \leqslant m<\ell .
\end{gathered}
$$


Тогда имеют место следующие соотношения:

$$
\begin{gathered}
c_{k, i} b_{k, i}=d_{k, i} a_{k+1, i}, \quad a_{k, i} d_{k-1, i-1}=b_{k, i} c_{k, i-1}, \quad 1 \leqslant i \leqslant k<\ell ; \\
b_{\ell, 1} c_{\ell, 1}=e^{2 z_{\ell, 1}}, \quad c_{\ell, i} b_{\ell, i}=e^{z_{\ell, i}-z_{\ell, i-1}}, \quad i=2, \ldots, \ell .
\end{gathered}
$$

Эти равенства можно рассматривать как соотношения между элементарными путями на диаграмме Гивенталя. Используя соотношения для более общих путей, которые следуют из (1.172), мы можем определить торическое вырождение

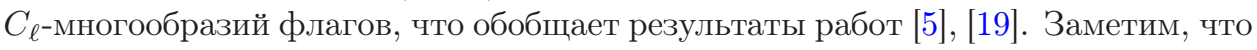
указанная диаграмма совпадает с диаграммой Гельфанда-Цейтлина для алгебры Ли $\mathfrak{s p}_{2 \ell}$.

1.4.3. Рекурсия для $\mathfrak{s p}_{2 \ell}$-функций Уиттекера и $\mathscr{Q}$-оператор для $A_{2 \ell-1}^{(2)}$-цепочки Тоды. Интегральное представление (1.165), (1.166) для $\mathfrak{s p}_{2 \ell}$-функций Уиттекера обладает рекурсивной структурой относительно ранга $\ell$. При $n=$ $2, \ldots, \ell$ определим интегральные операторы $Q_{C_{n-1}}^{C_{n}}$ с интегральными ядрами

$$
\begin{gathered}
Q_{C_{n-1}}^{C_{n}}\left(\underline{z}_{n}, \underline{z}_{n-1} ; \lambda_{n}\right)=\int d \underline{x}_{n} Q_{D_{n}}^{C_{n}}\left(\underline{z}_{n}, \underline{x}_{n}\right) Q_{C_{n-1}^{D_{n}}}\left(\underline{x}_{n}, \underline{z}_{n-1}\right)\left(e^{z_{n, 1}}+e^{z_{n-1,1}}\right)^{\imath \lambda_{n}} \\
\times \exp \left\{-\imath \lambda_{n}\left(\sum_{i=1}^{n} z_{n, i}-x_{n, 1}-2 \sum_{i=2}^{n} x_{n, i}+\sum_{i=1}^{n-1} z_{n-1, i}\right)\right\}
\end{gathered}
$$

где

$$
\begin{aligned}
Q_{C_{n-1}}^{D_{n}}\left(\underline{x}_{n}, \underline{z}_{n-1}\right) & =\exp \left\{-e^{x_{n, 1}+z_{n-1,1}}-\sum_{i=1}^{n-1}\left(e^{z_{n-1, i}-x_{n, i}}+e^{x_{n, i+1}-z_{n-1, i}}\right)\right\}, \\
Q_{D_{n}}^{C_{n}}\left(\underline{z}_{n}, \underline{x}_{n}\right) & =\exp \left\{-e^{x_{n, 1}+z_{n, 1}}-\sum_{i=1}^{n-1}\left(e^{z_{n, i}-x_{n, i}}+e^{x_{n, i+1}-z_{n, i}}\right)-e^{z_{n, n}-x_{n, n}}\right\} .
\end{aligned}
$$

При $n=1$ положим

$$
Q_{C_{0}}^{C_{1}}=\int d x_{1,1} e^{\imath \lambda_{1} x_{1,1}} \exp \left\{-\left(e^{x_{1,1}+z_{1,1}}+e^{z_{1,1}-x_{1,1}}\right)\right\} .
$$

Используя операторы $Q_{C_{n-1}}^{C_{n}}$, интегральное представление для $\mathfrak{s p}_{2 \ell}$-функции Уиттекера можно записать в рекурсивной форме.

Теорема 1.11. Интегральные представления для собственных функиий $\mathfrak{s p}_{2 \ell}$-цепочки Тоды (1.165) имеют вид

$$
\Psi_{\lambda_{1}, \ldots, \lambda_{\ell}}^{C_{\ell}}\left(z_{1}, \ldots, z_{\ell}\right)=\int_{C} \prod_{k=1}^{\ell-1} d \underline{z}_{k} \prod_{n=1}^{\ell} Q_{C_{n-1}}^{C_{n}}\left(\underline{z}_{n}, \underline{z}_{n} ; \lambda_{n}\right),
$$

или, эквивалентно,

$$
\Psi_{\lambda_{1}, \ldots, \lambda_{\ell}}^{C_{\ell}}\left(\underline{z}_{\ell}\right)=\int_{\mathscr{C}_{\ell}} d \underline{z}_{\ell-1} Q_{C_{\ell-1}}^{C_{\ell}}\left(\underline{z}_{\ell}, \underline{z}_{\ell-1} ; \lambda_{\ell}\right) \Psi_{\lambda_{1}, \ldots, \lambda_{\ell-1}}^{C_{\ell-1}}\left(\underline{z}_{\ell-1}\right) .
$$


Здесъ $z_{i}:=z_{\ell, i}, i=1, \ldots, \ell, u C \subset N_{+}$- некомпактное подмногообразие половинной размерности, для которого подынтегральное выражение экспоненииалъно убъвает на границе и на бесконечности.

Эта рекурсивная форма интегрального представления аналогична случаю $\mathfrak{s o}_{2 \ell+1}$. Но в отличие от $\mathfrak{g l}_{\ell+1}$-случая $(1.54)$, ее рекурсивное ядро $Q_{C_{n-1}}^{C_{n}}$ задано нетривиальным интегралом. Подобно $\mathfrak{s o}_{2 \ell+1}$-функции Уиттекера, при рассмотрении функции Уиттекера на нулевом уровне спектра $\left\{\lambda_{i}=0\right\}$ возникает новая структура. Как следует из (1.173), ядра $Q_{C_{n-1}}^{C_{n}}$ при $\lambda_{n}=0$ задаются свертками ядер $Q_{D_{n}}^{C_{n}}\left(\underline{z}_{n}, \underline{x}_{n}\right)$ и $Q_{C_{n-1}}^{D_{n}}\left(\underline{x}_{n}, \underline{z}_{n-1}\right)$. Соответствующие интегральные операторы $Q_{D_{n}}^{C_{n}}, Q_{C_{n-1}}^{D_{n}}$ можно рассматривать как элементарные сплетающие операторы, связывающие гамильтонианы цепочек Тоды для систем корней типов $C_{n}$, $D_{n}$ и $D_{n}, C_{n-1}$ соответственно. Например, легко проверяются сплетающие со-

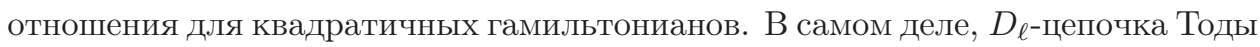
имеет следующие квадратичные гамильтонианы (более подробное обсуждение можно найти в п. 2.4.3):

$$
\mathscr{H}_{2}^{D_{\ell}}\left(\underline{x}^{(\ell)}\right)=-\frac{1}{2} \sum_{i=1}^{\ell} \frac{\partial^{2}}{\partial x_{i}^{2}}+e^{x_{1}+x_{2}}+\sum_{i=1}^{\ell-1} e^{x_{i+1}-x_{i}} .
$$

ПредложениЕ 1.10. Интегралънъе операторь $Q_{C_{n-1}}^{C_{n}}, Q_{D_{n}}^{C_{n}} u Q_{C_{n-1}}^{D_{n}}$ удовлетворяют следующим соотношениям.

1. Операторь $Q_{D_{n}}^{C_{n}}$ и $Q_{C_{n-1}}^{D_{n}}$ cплетают квадратичные гамильтонианы $C$ u D-uепочек Tоды:

$$
\begin{aligned}
\mathscr{H}_{2}^{D_{n}}\left(\underline{x}_{n}\right) Q_{C_{n-1}}^{D_{n}}\left(\underline{x}_{n}, \underline{z}_{n-1}\right) & =Q_{C_{n-1}}^{D_{n}}\left(\underline{x}_{n}, \underline{z}_{n-1}\right) \mathscr{H}_{2}^{C_{n-1}}\left(\underline{x}_{n-1}\right), \\
\mathscr{H}_{2}^{C_{n}}\left(\underline{z}_{n}\right) Q_{D_{n}}^{C_{n}}\left(\underline{z}_{n}, \underline{x}_{n}\right) & =Q_{D_{n}}^{C_{n}}\left(\underline{z}_{n}, \underline{x}_{n}\right) \mathscr{H}_{2}^{D_{n}}\left(\underline{x}_{n}\right) .
\end{aligned}
$$

2. Onератор $Q_{C_{n-1}}^{C_{n}}$ nри $\lambda_{n}=0$ cплетает гамильтонианы $\mathscr{H}_{2}^{C_{n}}$ и $\mathscr{H}_{2}^{C_{n-1}}$ :

$$
\mathscr{H}_{2}^{C_{n}}\left(\underline{z}_{n}\right) Q_{C_{n-1} C_{n}}\left(\underline{z}_{n}, \underline{z}_{n-1}\right)=Q_{C_{n-1}}^{C_{n}}\left(\underline{z}_{n}, \underline{z}_{n-1}\right) \mathscr{H}_{2}^{C_{n-1}}\left(\underline{z}_{n-1}\right) .
$$

Интегральное ядро оператора $Q_{C_{n-1}}^{C_{n}}$ может быть задано следующей диаграммой:

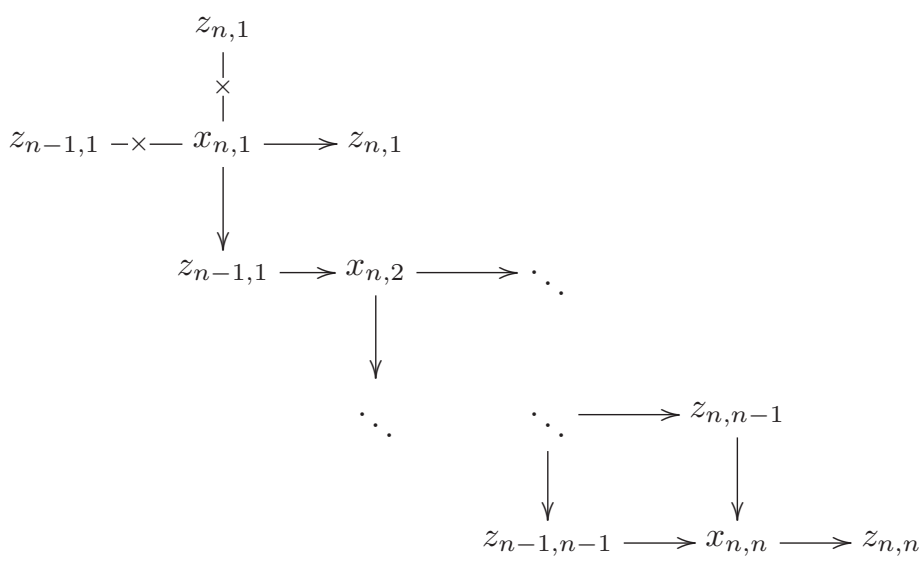


Здесь верхняя (нижняя) граница ориентированной диаграммы отвечает ядрам элементарных сплетающих операторов $Q_{D_{n}}^{C_{n}}\left(Q_{C_{n-1}}^{D_{n}}\right)$ и свертка двух ядер определяется интегрированием по переменным $x_{n, 1}, \ldots, x_{n, n}$ на диагонали диаграммы.

Подобно случаям $\mathfrak{g l}_{\ell+1}$ и $\mathfrak{s p}_{2 \ell}$, операторы рекурсии $Q_{C_{n-1}}^{C_{n}}$ можно рассматривать как вырождения $\mathscr{Q}$-операторов Бакстера для скрученной аффинной $A_{2 \ell-1}^{(2)}$-цепочки Тоды, которая определяется ниже. Подчеркнем, что до настоящего момента $\mathscr{Q}$-операторы для $A_{2 \ell-1}^{(2)}$ не были известны. Мы не будем выписывать здесь полный набор характеристических свойств введенных $\mathscr{Q}$-операторов и ограничимся рассмотрением коммутационных соотношений для гамильтонианов квадратичных аффинных цепочек Тоды. Подробное обсуждение этого вопроса будет дано в другом месте.

Начнем с описания $A_{2 \ell-1}^{(2)}$-цепочек Тоды. Множество простых корней аффинной системы корней $A_{2 \ell-1}^{(2)}$ можно записать в ортонормированном базисе $\left\{\epsilon_{i}, i=1, \ldots, \ell\right\}$ пространства $\mathbb{R}^{\ell}$ следующим образом:

$$
\alpha_{1}=2 \epsilon_{1}, \quad \alpha_{i+1}=\epsilon_{i+1}-\epsilon_{i}, \quad 1 \leqslant i \leqslant \ell-1, \quad \alpha_{\ell+1}=-\epsilon_{\ell}-\epsilon_{\ell-1} .
$$

Соответствующая диаграмма Дынкина имеет вид

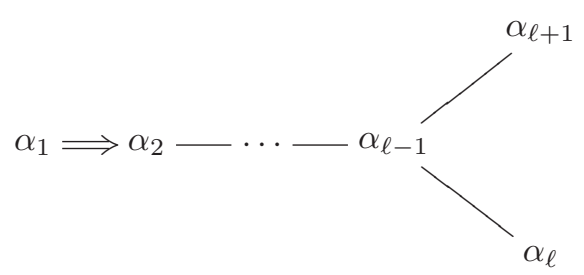

Эти корневые данные позволяют определить аффинную $A_{2 \ell-1}^{(2)}$-цепочку Тоды с квадратичным гамильтонианом

$$
\mathscr{H}_{2}^{A_{2 \ell-1}^{(2)}}\left(\underline{z}^{(\ell)}\right)=-\frac{1}{2} \sum_{i=1}^{\ell} \frac{\partial^{2}}{\partial z_{i}^{2}}+2 e^{2 z_{1}}+\sum_{i=1}^{\ell-1} e^{z_{i+1}-z_{i}}+g e^{-z_{\ell-1}-z_{\ell}}
$$

где $g$ - произвольный параметр.

Определим $\mathscr{Q}$-оператор Бакстера $A_{2 \ell-1}^{(2)}$-цепочки Тоды как интегральный оператор с интегральным ядром

$$
\begin{aligned}
& \mathscr{Q}^{A_{2 \ell-1}^{(2)}}\left(\underline{z}^{(\ell)}, \underline{y}^{(\ell)} ; \lambda\right)=\int d \underline{x}^{(\ell)} Q_{A_{2 \ell-1}^{(2)}\left(\underline{z}^{(\ell)}, \underline{x}^{(\ell)}\right) Q_{A_{2 \ell-1}^{(2)}}^{A_{2 \ell-1}^{(2)}}\left(\underline{x}^{(\ell)}, \underline{y}^{(\ell)}\right)} \\
& \quad \times \exp \left\{-\imath \lambda\left(\sum_{i=1}^{\ell} z_{i}-x_{1}-2 \sum_{i=2}^{\ell} x_{i}+\sum_{i=1}^{\ell} y_{i}\right)\right\}\left(e^{z_{1}}+e^{y_{1}}\right)^{\imath \lambda}\left(e^{-z_{\ell}}+e^{-y_{\ell}}\right)^{-2 \imath \lambda},
\end{aligned}
$$

где

$$
Q_{A_{2 \ell-1}^{(2)-1}}^{A_{(2)}^{(2)}}\left(\underline{z}^{(\ell)}, \underline{x}^{(\ell)}\right)=\exp \left\{-e^{z_{1}+x_{1}}-\sum_{i=1}^{\ell-1}\left(e^{z_{i}-x_{i}}+e^{x_{i+1}-z_{i}}\right)-e^{z_{\ell}-x_{\ell}}-g e^{-z_{\ell}-x_{\ell}}\right\}
$$


$Q_{A_{2 \ell-1}^{(2)}}^{A_{2 \ell}^{(2)}}\left(\underline{x}^{(\ell)}, \underline{y}^{(\ell)}\right)=\exp \left\{-e^{y_{1}+x_{1}}-\sum_{i=1}^{\ell-1}\left(e^{y_{i}-x_{i}}+e^{x_{i+1}-y_{i}}\right)-e^{y_{\ell}-x_{\ell}}-g e^{-y_{\ell}-x_{\ell}}\right\}$.

Здесь мы используем обозначения $\underline{z}^{(\ell)}=\left(z_{1}, \ldots, z_{\ell}\right), \underline{y}^{(\ell)}=\left(y_{1}, \ldots, y_{\ell}\right)$.

Следующее утверждение может быть проверено непосредственно.

ПредЛОЖЕНИЕ 1.11. Q-оператор (1.184) коммутирует с квадратичным гамильтонианом $A_{2 \ell-1}^{(2)}$-цепочки Тодъ:

$$
\mathscr{H}^{A_{2 \ell-1}^{(2)}}\left(\underline{z}^{(\ell)}\right) Q^{A_{2 \ell-1}^{(2)}}\left(\underline{z}^{(\ell)}, \underline{y}^{(\ell)}\right)=Q^{A_{2 \ell-1}^{(2)}}\left(\underline{z}^{(\ell)}, \underline{y}^{(\ell)}\right) \mathscr{H}^{A_{2 \ell-1}^{(2)}}\left(\underline{y}^{(\ell)}\right) .
$$

Покажем, что оператор рекурсии $Q_{C_{\ell-1}}^{C_{\ell}}$ может быть получен вырождением Q-оператора Бакстера для $A_{2 \ell-1}^{(2)}$. Рассмотрим несколько модифицированный оператор рекурсии $Q_{C_{\ell-1} \oplus C_{1}}^{C_{\ell}}$ с ядром

$$
Q_{C_{\ell-1} \oplus C_{1}}^{C_{\ell}}\left(\underline{z}^{(\ell)}, \underline{y}^{(\ell)} ; \lambda\right):=e^{\imath \lambda y_{\ell}} Q_{C_{\ell-1}}^{C_{\ell}}\left(\underline{z}^{(\ell)}, \underline{y}^{(\ell-1)} ; \lambda\right),
$$

где $\underline{y}^{(\ell-1)}=\left(y_{1}, \ldots, y_{\ell-1}\right)$. Так определенный оператор сплетает гамильтонианы $\overline{s p}_{2 \ell^{-}}$и $\mathfrak{s p}_{2 \ell-2} \oplus \mathfrak{s p}_{2}$-цепочек Тоды. Тем самым, для квадратичных гамильтонианов мы имеем

$$
\begin{aligned}
\left.\mathscr{H}_{2}^{C_{\ell}}\left(\underline{z}^{(\ell)}\right) Q_{C_{\ell-1} \oplus C_{1}}^{C_{\ell}}\left(\underline{z}^{(\ell)}, \underline{y}^{(\ell)} ; \lambda\right)=Q_{C_{\ell-1} \oplus C_{1}}^{C_{\ell}} \underline{z}^{(\ell)}, \underline{y}^{(\ell)} ; \lambda\right) \\
\times\left(\mathscr{H}_{2}^{C_{\ell}}\left(\underline{y}^{(\ell-1)}\right)+\mathscr{H}_{2}^{C_{1}}\left(y_{\ell}\right)\right),
\end{aligned}
$$

где $\mathscr{H}_{2}^{C_{1}}\left(y_{\ell}\right)=-\frac{1}{2} \frac{\partial^{2}}{\partial y_{\ell}^{2}}$. Очевидно, что проекция указанного выше уравнения на подпространство функций $F\left(\underline{y}^{(\ell)}\right)=\exp \left(\imath \lambda y_{\ell}\right) f\left(\underline{y}^{(\ell-1)}\right)$ приводит к исходному оператору рекурсии, удовлетворяющему условиям

$$
\mathscr{H}_{2}^{C_{\ell}}\left(\underline{z}^{(\ell)}\right) Q_{C_{\ell-1}}^{C_{\ell}}\left(\underline{z}^{(\ell)}, \underline{y}^{(\ell-1)} ; \lambda\right)=Q_{C_{\ell-1}}^{C_{\ell}}\left(\underline{z}^{(\ell)}, \underline{y}^{(\ell-1)} ; \lambda\right)\left(\mathscr{H}_{2}^{C_{\ell}}\left(\underline{y}^{(\ell-1)}\right)+\frac{1}{2} \lambda^{2}\right) .
$$

Рассмотрим однопараметрическое семейство ядер:

$$
\begin{gathered}
\mathscr{Q}^{A_{2 \ell-1}^{(2)}}\left(\underline{z}^{(\ell)}, \underline{y}^{(\ell)} ; \lambda ; \varepsilon\right)=\varepsilon^{\imath \lambda} e^{\imath \lambda y \ell} \int d \underline{x}^{(\ell+1)} Q_{A_{2 \ell-1}^{(2)}}^{A_{2 \ell-1}^{(2)}}\left(\underline{z}^{(\ell)}, \underline{x}^{(\ell+1)}\right) Q_{A_{2 \ell-1}^{(2)}\left(\underline{x}^{(\ell+1)}, \underline{y}^{(\ell)} ; \varepsilon\right)}^{A^{(2)}} \\
\quad \times \exp \left\{-\imath \lambda\left(\sum_{i=1}^{\ell} z_{i}-x_{1}-2 \sum_{i=2}^{\ell} x_{i}+\sum_{i=1}^{\ell-1} y_{i}\right)\right\}\left(e^{z_{1}}+e^{y_{1}}\right)^{\imath \lambda}\left(\varepsilon e^{y_{\ell}-z_{\ell}}+1\right)^{-2 \imath \lambda}
\end{gathered}
$$

где

$$
\begin{aligned}
Q_{A_{2 \ell-1}^{(2)}}^{A_{2 \ell-1}^{(2)}}\left(\underline{x}^{(\ell+1)}, \underline{y}^{(\ell)} ; \varepsilon\right)=\exp \{ & -e^{y_{1}+x_{1}}-\sum_{i=1}^{\ell-1}\left(e^{y_{i}-x_{i}}+e^{x_{i+1}-y_{i}}\right) \\
& \left.-\varepsilon e^{y_{\ell}-x_{\ell}}-\varepsilon^{-1} g e^{-y_{\ell}-x_{\ell}}\right\},
\end{aligned}
$$


получено сдвигом переменной $y_{\ell}=y_{\ell}+\ln \varepsilon$ в (1.184)-(1.186). Тогда выполнено следующее соотношение между $\mathscr{Q}$-оператором для $A_{2 \ell-1}^{(2)}$-цепочки Тоды и оператором рекурсии для $\mathfrak{s p}_{2 \ell}$-функции Уиттекера:

$$
\left.Q_{C_{\ell-1} \oplus C_{1}}^{C_{\ell}} \underline{z}^{(\ell)}, \underline{y}^{(\ell)} ; \lambda\right)=\lim _{\substack{\varepsilon \rightarrow 0, \varepsilon^{-1} g \rightarrow 0}} \varepsilon^{-\imath \lambda} \mathscr{Q}^{A_{2 \ell-1}^{(2)}}\left(\underline{z}^{(\ell)}, \underline{y}^{(\ell)} ; \lambda ; \varepsilon\right) .
$$

1.5. Интегральные представления собственных функций $\mathfrak{s o}_{2 \ell}$-цепочки Тоды. В этом разделе мы получим аналог интегрального представления Гивенталя функций Уиттекера для алгебр Ли $\mathfrak{s o}_{2 \ell}$. Как и в рассмотренных выше случаях, мы начнем с интегрального представления $\mathfrak{s o}_{2 \ell}$-функций Уиттекера, использующего факторизационную параметризацию. Затем мы определяем модификацию факторизационной параметризации, которая приводит к интегральным представлениям типа Гивенталя для $\mathfrak{s o}_{2 \ell}$-функций Уиттекера.

Рассмотрим систему корней $D_{\ell}$, которая отвечает алгебре Ли $\mathfrak{s o}_{2 \ell}$. Пусть $\left\{\epsilon_{1}, \ldots, \epsilon_{\ell}\right\}$ - ортонормированный базис в $\mathbb{R}^{\ell}$. Мы реализуем простые корни и фундаментальные веса для системы корней $D_{\ell}$ как следующие векторы в $\mathbb{R}^{\ell}$ :

$$
\begin{array}{ll}
\alpha_{1}=\epsilon_{2}-\epsilon_{1}, & \omega_{1}=\frac{1}{2}\left(-\epsilon_{1}+\epsilon_{2}+\cdots+\epsilon_{\ell}\right), \\
\alpha_{2}=\epsilon_{2}+\epsilon_{1}, & \omega_{2}=\frac{1}{2}\left(\epsilon_{1}+\epsilon_{2}+\cdots+\epsilon_{\ell}\right), \\
\alpha_{3}=\epsilon_{3}-\epsilon_{2}, & \omega_{3}=\epsilon_{3}+\cdots+\epsilon_{\ell}, \\
\cdots & \ldots \\
\alpha_{n}=\epsilon_{\ell}-\epsilon_{\ell-1}, & \omega_{\ell}=\epsilon_{\ell} .
\end{array}
$$

Кокорни $\alpha_{i}^{\vee}$ можно отождествить с соответствующими корнями $\alpha_{i}$, используя стандартное скалярное произведение в $\mathbb{R}^{\ell}$. Свяжем с этими корневыми данными $\mathfrak{s o}_{2 \ell}$-цепочку Тоды с квадратичным гамильтонианом

$$
\mathscr{H}_{2}^{D_{\ell}}=-\frac{1}{2} \sum_{i=1}^{\ell} \frac{\partial^{2}}{\partial x_{i}^{2}}+e^{x_{1}+x_{2}}+\sum_{i=1}^{\ell-1} e^{x_{i+1}-x_{i}} .
$$

Можно дополнить (1.193) до полного набора из $\ell$ попарно коммутирующих функционально независимых гамильтонианов $\mathscr{H}_{k}^{D_{\ell}}$ для $\mathfrak{s o}_{2 \ell}$-цепочки Тоды. Мы хотим получить интегральные представления для общих собственных функций этого полного набора гамильтонианов. Соответствующая задача о собственной функции для квадратичного гамильтониана может быть записана в виде:

$$
\mathscr{H}_{2}^{D_{\ell}} \Psi_{\lambda_{1}, \ldots, \lambda_{\ell}}^{D_{\ell}}\left(x_{1}, \ldots, x_{\ell}\right)=\frac{1}{2} \sum_{i=1}^{\ell} \lambda_{i}^{2} \Psi_{\lambda_{1}, \ldots, \lambda_{\ell}}^{D_{\ell}}\left(x_{1}, \ldots, x_{\ell}\right) .
$$

1.5.1. $\mathfrak{s o}_{2 \ell}$-функция Уиттекера: факторизационная параметризация. Приведенное слово, отвечающее элементу максимальной длины $w_{0}$ в группе Вейля системы корней алгебры Ли $\mathfrak{s o}_{2 \ell}$, можно представить в следующей рекурсивной форме:

$$
I_{\ell}=\left(i_{1}, i_{2}, \ldots, i_{m_{\ell}}\right):=(12,3123, \ldots,(\ell \ldots 3123 \ldots \ell)), \quad m_{\ell}=\ell(\ell-1),
$$


где индексы $i_{k} \in \Gamma=\{1, \ldots, \ell\}$ соответствуют отражениям относительно простых корней $\alpha_{i_{k}}$. Пусть $N_{+} \subset G$ - максимальная унипотентная подгруппа в $G=\mathrm{SO}(2 \ell)$. Свяжем с приведенным словом $I_{\ell}$ следующую рекурсивную параметризацию типичного элемента $v^{D_{\ell}} \in N_{+}$:

$$
v^{D_{\ell}}=v^{D_{\ell-1}} \mathfrak{X}_{D_{\ell-1}}^{D_{\ell}},
$$

где

$$
\begin{aligned}
\mathfrak{X}_{D_{\ell-1}}^{D_{\ell}}= & X_{\ell}\left(y_{\ell, 1}\right) \cdots X_{k}\left(y_{k, 2(\ell+1-k)-1}\right) \cdots X_{3}\left(y_{3,2 \ell-5}\right) X_{1}\left(y_{1, \ell-1}\right) \cdots \\
& \times X_{2}\left(y_{2, \ell-1}\right) X_{3}\left(y_{3,2 \ell-4}\right) \cdots X_{k}\left(y_{k, 2(\ell+1-k)}\right) \cdots X_{\ell}\left(y_{\ell, 2}\right) .
\end{aligned}
$$

Здесь $X_{i}(y)=e^{y f_{i}}$ и $f_{i} \equiv f_{\alpha_{i}}$ - образующие, отвечающие простым корням. Подмножество $N_{+}^{(0)}$ элементов, допускающих параметризацию (1.195), (1.196), открыто в $N_{+}$. Действие алгебры Ли $\mathfrak{s o}_{2 \ell}$ на $N_{+}(1.10)$ определяет действие на пространстве функций на $N_{+}^{(0)}$. Явное описание действия на пространстве $V_{\mu}$ эквивариантных функций на $N_{+}^{(0)}$ приведено ниже.

ПреДложЕниЕ 1.12. Следующие дифференииальные операторы определяют реализацию представления основной серии $\pi_{\mu}$ алгебры $\mathscr{U}\left(\mathfrak{s o}_{2 \ell}\right)$ в пространстве $V_{\mu}$ в терминах факторизационной параметризации на $N_{+}^{(0)}$ :

$$
\begin{aligned}
& F_{i}=\frac{\partial}{\partial y_{i, \ell-1}}+\sum_{n=1}^{\alpha(\ell)-1} \prod_{k=2 n-1}^{\ell-2} \frac{y_{3,2 k}}{y_{3,2 k-1}} \prod_{j=n}^{\alpha(\ell)-1} \frac{y_{i, 2 j+\varpi(\ell)}}{y_{i, 2 j+1+\varpi(\ell)}} \frac{y_{i^{*}, 2 j+1+\varpi(\ell)}}{y_{i^{*}, 2 j+\varpi(\ell)}} \\
& \times\left\{\frac{\partial}{\partial y_{i, 2 n-1+\varpi(\ell)}}-\frac{\partial}{\partial y_{i, 2 n+\varpi(\ell)}}\right\}+\sum_{n=1}^{\beta(\ell)-1} \prod_{k=2 n-\varpi(\ell)}^{\ell-2} \frac{y_{3,2 k}}{y_{3,2 k-1}} \frac{y_{i^{*}, \ell-1}}{y_{i, \ell-1}} \\
& \times \prod_{j=n+1}^{\beta(\ell)-1} \frac{y_{i, 2 j-\varpi(\ell)}}{y_{i, 2 j-1-\varpi(\ell)}} \frac{y_{i^{*}, 2 j-1-\varpi(\ell)}}{y_{i^{*}, 2 j-\varpi(\ell)}}\left\{\frac{\partial}{\partial y_{i^{*}, 2 n-\varpi(\ell)}}-\frac{\partial}{\partial y_{i, 2 n+1-\varpi(\ell)}}\right\} \\
& +\sum_{n=1}^{\ell-2} \frac{y_{3,2(\ell-2)}}{i, \ell-1} \prod_{k=n}^{\ell-3} \frac{y_{3,2 k}}{y_{3,2 k+1}} \prod_{j=\alpha(n+\varpi(\ell))}^{\alpha(\ell)-1} \frac{y_{i^{*}, 2 j+1+\varpi(\ell)}}{y_{i^{*}, 2 j+\varpi(\ell)}} \\
& \times \prod_{m=\beta(n+\varpi(\ell))}^{\beta(\ell)-2} \frac{y_{i, 2 m+2-\varpi(\ell)}}{y_{i, 2 m+1-\varpi(\ell)}}\left\{\frac{\partial}{\partial y_{3,2 n-1}}-\frac{\partial}{\partial y_{3,2 n}}\right\}
\end{aligned}
$$

nрu $i=1,2 u *: 1 \leftrightarrow 2$,

$$
\begin{aligned}
F_{k}=\frac{\partial}{\partial y_{k, 2(\ell+1-k)}} & \\
& +\left(1-\delta_{k, \ell)}\right) \sum_{n=1}^{\ell-k}\left\{\prod_{j=n}^{\ell-k} \frac{y_{k+1,2 j}}{y_{k+1,2 j-1}} \frac{y_{k, 2 j+1}}{y_{k, 2 j+2}}\left(\frac{\partial}{\partial y_{k, 2 n}}-\frac{\partial}{\partial y_{k, 2 n+1}}\right)\right. \\
& \left.+\frac{y_{k+1,2(\ell-k)}}{y_{k, 2(\ell+1-k)}} \prod_{j=n}^{\ell-1} \frac{y_{k+1,2 j}}{y_{k+1,2 j+1}} \frac{y_{k, 2 j+3}}{y_{k, 2 j+2}}\left(\frac{\partial}{\partial y_{k+1,2 n-1}}-\frac{\partial}{\partial k+1,2 n}\right)\right\}
\end{aligned}
$$


npu $k=3, \ldots, \ell$,

$$
H_{k}=\left\langle\mu, \alpha_{k}^{\vee}\right\rangle+\sum_{i=1}^{\ell} a_{k, i} \sum_{j=1}^{n_{i}} y_{i, j} \frac{\partial}{\partial y_{i, j}}
$$

npu $k=1, \ldots, \ell$,

$$
E_{i}=\sum_{n=1}^{\ell-1} y_{i, n}\left\{-\left\langle\mu, \alpha_{i}^{\vee}\right\rangle-2 \sum_{k=1}^{n-1} y_{i, k} \frac{\partial}{\partial y_{i, k}}+\sum_{k=1}^{2 n-3} y_{3, k} \frac{\partial}{\partial y_{3, k}}-y_{i, n} \frac{\partial}{\partial y_{i, n}}\right\}
$$

при $i=1,2$,

$$
\begin{aligned}
E_{3}= & \sum_{n=1}^{2(\ell-2)} y_{3, n}\left\{\mu_{2}-\mu_{3}+\sum_{k=1}^{\alpha(n)+1}\left(y_{1, k} \frac{\partial}{\partial y_{1, k}}+y_{2, k} \frac{\partial}{\partial y_{2, k}}\right)\right. \\
& \left.+\sum_{k=1}^{2 \beta(n)-3} y_{4, k} \frac{\partial}{\partial y_{4, k}}-2 \sum_{k=1}^{n-1} y_{3, k} \frac{\partial}{\partial y_{3, k}}-y_{3, n} \frac{\partial}{\partial y_{3, n}}\right\}, \\
E_{k}^{(\ell)}= & \sum_{n=1}^{2(\ell+1-k)} y_{k, n}\left\{\mu_{k-1}-\mu_{k}+\sum_{j=1}^{2 \alpha(n)+2} y_{k-1, j} \frac{\partial}{\partial y_{k-1, j}}+\sum_{j=1}^{2 \beta(n)-3} y_{k+1, j} \frac{\partial}{\partial y_{k+1, j}}\right. \\
& \left.-2 \sum_{j=1}^{n-1} y_{k, j} \frac{\partial}{\partial y_{k, j}}-y_{k, n} \frac{\partial}{\partial y_{k, n}}\right\}, \quad k=4, \ldots, \ell,
\end{aligned}
$$

где $\alpha(n):=[n / 2], \beta(n):=[(n+1) / 2] u \varpi(n)=\left(1-(-1)^{n}\right) / 2$.

Доказательство приведено в п. 2.4.4 части 2 .

Левый и правый векторы Уиттекера, определенные в (1.7) и (1.8), могут быть найдены в факторизационной параметризации явно.

Лемма 1.9. Имеют место следующие выражения для левого и правого векторов Уиттекера:

$$
\begin{aligned}
\psi_{R}(y)= & \exp \left\{-\left(\sum_{n=1}^{\ell-1} y_{1, n}+\sum_{n=1}^{\ell-1} y_{2, n}+\sum_{k=3}^{\ell} \sum_{n=1}^{2(\ell+1-k)} y_{k, n}\right)\right\}, \\
\psi_{L}(y)=( & \left.\prod_{k=1}^{\ell-1} y_{1, k}^{\varpi(k)} y_{2, k}^{1-\varpi(k)} \prod_{n=3}^{\ell} \prod_{i=3}^{n} y_{i, 2(n+1-i)-1}\right)^{\left\langle\mu, \alpha_{1}^{\vee}\right\rangle} \\
& \times\left(\prod_{k=1}^{\ell-1} y_{1, k}^{1-\varpi(k)} y_{2, k}^{\varpi(k)} \prod_{n=3}^{\ell} \prod_{i=3}^{n} y_{i, 2(n+1-i)-1}\right)^{\left\langle\mu, \alpha_{2}^{\vee}\right\rangle} \\
& \times \prod_{k=3}^{\ell}\left(\prod_{i=1}^{k} \prod_{n=1}^{n_{i}} y_{i, n} \prod_{i=k+1}^{\ell} \prod_{n=1}^{n_{i} / 2} y_{i, 2 n-1}^{2}\right)^{\left\langle\mu, \alpha_{k}^{\vee}\right\rangle} \\
& \times \exp \left\{-\left(\sum _ { n = 1 } ^ { \ell - 1 } \left[\frac{1}{y_{1, \ell-1}} \prod_{k=1}^{n-1}\left(\frac{y_{1, \ell-k}}{y_{1, \ell-k-1}}\right)^{1-\varpi(k)}\left(\frac{y_{2, \ell-k}}{y_{2, \ell-k-1}}\right)^{\varpi(k)}\right.\right.\right. \\
& \left.+\frac{1}{y_{2, \ell-1}} \prod_{k=1}^{n-1}\left(\frac{y_{1, \ell-k}}{y_{1, \ell-k-1}}\right)^{\varpi(k)}\left(\frac{y_{2, \ell-k}}{y_{2, \ell-k-1}}\right)^{1-\varpi(k)}\right]\left(1+\frac{y_{3,2(\ell-n-1)}}{y_{3,2(\ell-n-1)-1}}\right)
\end{aligned}
$$




$$
\begin{aligned}
& \times \prod_{k=1}^{n-1} \frac{y_{3,2(\ell-k-1)}}{y_{3,2(\ell-k-1)-1}}+\sum_{k=3}^{\ell} \frac{1}{y_{k, 2(\ell+1-k)}} \sum_{n=k}^{\ell}\left(1+\frac{y_{k+1,2(\ell-n)}}{y_{k+1,2(\ell-n)-1}}\right)^{1-\delta_{n, \ell}} \\
& \left.\left.\times \prod_{j=k}^{n-1} \frac{y_{k, 2(\ell-j)-1}}{y_{k, 2(\ell-j)}} \frac{y_{k+1,2(\ell-j)}}{y_{k+1,2(\ell-j)-1}}\right)\right\}
\end{aligned}
$$

где $\varpi(n)=\left(1-(-1)^{n}\right) / 2$ - четность числа $n$.

Доказательство приведено в п. 2.3.4 части 2.

Используя (1.17) и (1.18), несложно получить интегральные представления для $\mathfrak{s o}_{2 \ell}$-функции Уиттекера в факторизационной параметризации.

ТеОрема 1.12. Собственные функиии $\mathfrak{s o}_{2 \ell}$-цепочки Тоды допускают следующее интегральное представление:

$$
\begin{aligned}
\Psi_{\lambda_{1}, \ldots, \lambda_{\ell}}^{D_{\ell}} & \left(x_{1}, \ldots, x_{\ell}\right)=e^{\imath \lambda_{1} x_{1}+\cdots+\imath \lambda_{\ell} x_{\ell}} \int_{C} \prod_{i=1}^{\ell} \prod_{k=1}^{n_{i}} \frac{d y_{i, k}}{y_{i, k}} \\
& \times \prod_{k=1}^{\ell-1}\left(\frac{y_{1, k}}{y_{2, k}}\right)^{\imath \lambda_{1}(-1)^{k}}\left(\prod_{n=3}^{\ell} \prod_{i=3}^{n} y_{i, 2(n+1-i)-1}\right)^{2 \imath \lambda_{2}} \\
& \times \prod_{k=3}^{\ell}\left(\prod_{i=1}^{k} \prod_{n=1}^{n_{i}} y_{i, n} \prod_{i=k+1}^{\ell} \prod_{n=1}^{n_{i} / 2} y_{i, 2 n-1}^{2}\right)^{\imath\left(\lambda_{k}-\lambda_{k-1}\right)} \\
& \times \exp \left\{-\left(\sum _ { n = 1 } ^ { \ell - 1 } \left[\frac{1}{y_{1, \ell-1}} \prod_{k=1}^{n-1}\left(\frac{y_{1, \ell-k}}{y_{1, \ell-k-1}}\right)^{1-\varpi(k)}\left(\frac{y_{2, \ell-k}}{y_{2, \ell-k-1}}\right)^{\varpi(k)}\right.\right.\right. \\
& \left.+\frac{1}{y_{2, \ell-1}} \prod_{k=1}^{n-1}\left(\frac{y_{1, \ell-k}}{y_{1, \ell-k-1}}\right)^{\varpi(k)}\left(\frac{y_{2, \ell-k}}{y_{2, \ell-k-1}}\right)^{1-\varpi(k)}\right]\left(1+\frac{y_{3,2}(\ell-n-1)}{y_{3,2}(\ell-n-1)-1}\right) \\
& \times \prod_{k=1}^{n-1} \frac{y_{3,2(\ell-k-1)}}{y_{3,2(\ell-k-1)-1}}+\sum_{k=3}^{\ell} \frac{1}{y_{k, 2(\ell+1-k)}} \sum_{n=k}^{\ell}\left(1+\frac{y_{k+1,2(\ell-n)}}{y_{k+1,2(\ell-n)-1}}\right)^{1-\delta_{n, \ell}} \\
& \times \prod_{j=k}^{n-1} \frac{y_{k, 2(\ell-j)-1}}{y_{k, 2(\ell-j)}} \frac{y_{k+1,2(\ell-j)}}{y_{k+1,2(\ell-j)-1}+e^{x_{2}-x_{1}}} \sum_{n=1}^{\ell-1} y_{1, n}+e^{x_{1}+x_{2}} \sum_{n=1}^{\ell} y_{2, n} \\
& \left.\left.+\sum_{k=3}^{\ell} e^{x_{k}-x_{k-1}} \sum_{n=1} y_{k, n}\right)\right\} .
\end{aligned}
$$

Здесъ $\varpi(k)=\left(1-(-1)^{k}\right) / 2, n_{1}=n_{2}=\ell-1, n_{k}=2(\ell+1-k), k>2, u$ $m=l\left(w_{0}\right)$. Область интегрирования $C \subset N_{+}-$это некомпактное подмногообразие половинной размерности, для которого подынтегральное выражение экспоненциально убывает на гранище и на бесконечности. В частности, в качестве $C$ можно взять малую деформацию подмножества $\mathbb{R}_{+}^{\ell(\ell-1)}$ в $\mathbb{C}^{\ell(\ell-1)}$, обеспечивающую сходимость интеграла.

Доказательство приведено в п. 2.3.4 части 2. 
ПримеР 1.6. При $\ell=3$ общая формула (1.205) принимает вид

$$
\begin{aligned}
& \Psi_{\lambda_{1}, \lambda_{2}, \lambda_{3}}^{D_{3}}\left(x_{1}, x_{2}, x_{3}\right)=e^{\lambda_{1} x_{1}+\lambda_{2} x_{2}+\lambda_{3} x_{3}} \int_{C} \prod_{i=1}^{3} \prod_{k=1}^{2} \frac{d y_{i, k}}{y_{i, k}} \\
& \quad \times\left(y_{1,1} y_{3,1} y_{2,2}\right)^{\imath\left(\lambda_{2}-\lambda_{1}\right)}\left(y_{2,1} y_{3,1} y_{1,2}\right)^{\imath\left(\lambda_{2}+\lambda_{1}\right)}\left(y_{3,1} y_{1,2} y_{2,2} y_{3,2}\right)^{\imath\left(\lambda_{3}-\lambda_{2}\right)} \\
& \quad \times \exp \left\{\frac{1}{y_{1,2}}\left(1+\frac{y_{3,2}}{y_{3,1}}\right)+\frac{1}{y_{1,2}} \frac{y_{2,2}}{y_{2,1}} \frac{y_{3,2}}{y_{3,1}}+\frac{1}{y_{2,2}}\left(1+\frac{y_{3,2}}{y_{3,1}}\right)+\frac{1}{y_{2,2}} \frac{y_{1,2}}{y_{1,1}} \frac{y_{3,2}}{y_{3,1}}\right. \\
& \left.\quad+\frac{1}{y_{3,2}}+e^{x_{2}-x_{1}}\left(y_{1,1}+y_{1,2}\right)+e^{x_{2}+x_{1}}\left(y_{2,1}+y_{2,2}\right)+e^{x_{3}-x_{2}}\left(y_{3,1}+y_{3,2}\right)\right\} .
\end{aligned}
$$

1.5.2. $\mathfrak{s o}_{2 \ell}$-функция Уиттекера: модифицированная факторизационная параметризация. В этом пункте мы вводим модифицированную факторизационную параметризацию на открытом подмножестве $N_{+}^{(0)}$ максимальной унипотентной подгруппы $N_{+} \subset \mathrm{SO}(2 \ell)$. Эта параметризация используется для построения интегральных представлений для $\mathfrak{s o}_{2 \ell}$-функций Уиттекера. Подобно другим сериям классических алгебр Ли, эти интегральные представления для $\mathfrak{s o}_{2 \ell}$-функций Уиттекера имеют простую рекурсивную структуру относительно ранга $\ell$ и могут быть описаны в сугубо комбинаторных терминах с использованием подходящих графов. Эти представления можно рассматривать как обобщение интегральных представлений Гивенталя на случай $\mathfrak{g}=\mathfrak{s o}_{2 \ell}$.

Мы следуем тому же подходу, который был использован в описании модифицированной факторизационной параметризации для других классических групп. Имеется реализация тавтологического представления $\pi: \mathfrak{s o}_{2 \ell} \rightarrow \operatorname{End}\left(\mathbb{C}^{2 \ell}\right)$, для которой образующие Вейля, отвечающие борелевской (соответственно картановской) подалгебре в $\mathfrak{s o}_{2 \ell}$, переходят в верхнетреугольные (соответственно диагональные) матрицы. Это определяет вложение $\mathfrak{s o}_{2 \ell} \subset \mathfrak{g l}_{2 \ell}$, при котором борелевская (соответственно картановская) подалгебра отображается в борелевскую (соответственно картановскую) подалгебру (см., например, [17]). Чтобы определить соответствующее вложение групп, рассмотрим следующую инволюцию на $\mathrm{GL}(2 \ell)$ :

$$
g \longmapsto g^{*}:=\dot{W}_{0}^{-1}\left(g^{-1}\right)^{t} \dot{W}_{0},
$$

где $a^{t}$ индуцировано стандартным транспонированием матрицы $a$ и $\dot{W}_{0}-$ подъем элемента максимальной длины в группе Вейля $S_{2 \ell}$ алгебры $\mathfrak{g l}_{2 \ell}$. В матричной форме это можно записать как

$$
\pi\left(\dot{W}_{0}\right)=S J
$$

где $S=\operatorname{diag}(1,-1, \ldots, 1,-1)$ и $J=\left\|J_{i, j}\right\|=\left\|\delta_{i+j, 2 \ell+1}\right\|$. Тогда ортогональная группа $G=\mathrm{SO}(2 \ell)$ отождествляется с подгруппой в $\mathrm{GL}(2 \ell)$ (см., например, [17]):

$$
\mathrm{SO}(2 \ell)=\left\{g \in \mathrm{GL}(2 \ell): g^{*}=g\right\}
$$


Пусть $\epsilon_{i, j}$ - элементарные $(2 \ell \times 2 \ell)$-матрицы с единицей на месте $(i, j)$ и нулями на прочих местах. Рассмотрим следующие матрицы:

$$
\begin{aligned}
& U_{2}=\sum_{i=1}^{\ell} \epsilon_{i, i}+e^{-x_{1,1}} \epsilon_{\ell+1, \ell+1}+e^{x_{1,1}} \epsilon_{\ell+2, \ell+2}+\sum_{i=\ell+3}^{2 \ell} \epsilon_{i, i}, \\
& \widetilde{U}_{2}=U_{2}+e^{z_{1,1}}\left(2 \epsilon_{\ell, \ell+1}+\epsilon_{\ell+1, \ell+2}\right) \\
& U_{n}=\sum_{i=1}^{\ell} \epsilon_{i, i}+e^{-x_{n-1,1}} \epsilon_{\ell+1, \ell+1}+\sum_{k=1}^{n-1} e^{x_{n-1, k}} \epsilon_{\ell+1+k, \ell+1+k}+\sum_{i=\ell+1+n}^{2 \ell} \epsilon_{i, i}, \\
& \widetilde{U}_{n}=U_{n}+2 e^{z_{n-1,1}} \epsilon_{\ell, \ell+1}+\sum_{k=1}^{n-1} e^{z_{n-1, k}} \epsilon_{\ell+k, \ell+1+k}, \\
& \widetilde{U}_{n}^{\prime}=U_{n}+2 e^{z_{n-1,1}} \epsilon_{\ell, \ell+1}+e^{z_{n-1,1}} \epsilon_{\ell+1, \ell+2}, \\
& \widetilde{U}_{n}^{\prime \prime}=U_{n}+\sum_{k=2}^{n-1} e^{z_{n-1, k}} \epsilon_{\ell+k, \ell+1+k}
\end{aligned}
$$

при $n=3, \ldots, \ell$ и

$$
\begin{aligned}
& V_{2}=\sum_{i=1}^{\ell-1}\left(\epsilon_{i, i}+\epsilon_{\ell+1+i, \ell+1+i}\right)+e^{-x_{1,1}} \epsilon_{\ell, \ell}+e^{x_{1,1}} \epsilon_{\ell+1, \ell+1}, \\
& \tilde{V}_{2}=V_{2}+e^{z_{n-1,1}}\left(2 \epsilon_{\ell-1, \ell}+\epsilon_{\ell, \ell+1}\right) \\
& V_{n}=\sum_{i=1}^{\ell-n} \epsilon_{i, i}+e^{-x_{n, 1}} \epsilon_{\ell, \ell}+e^{x_{n, 1}} \epsilon_{\ell+1, \ell+1} \\
& \quad+\sum_{k=2}^{n} e^{x_{n, k}} \epsilon_{\ell+1-k, \ell+1-k}+\sum_{i=\ell+2}^{2 \ell} \epsilon_{i, i}, \\
& \tilde{V}_{n}=V_{n}+e^{z_{n-1,1}} \epsilon_{\ell, \ell+1}+\sum_{k=1}^{n-1}\left(1+\delta_{k, 1}\right) e^{z_{n-1, k}} \epsilon_{\ell-k, \ell+1-k}, \\
& \tilde{V}_{n}^{\prime}=V_{n}+e^{z_{n-1,1}}\left(2 \epsilon_{\ell-1, \ell}+\epsilon_{\ell, \ell+1}\right) \\
& \tilde{V}_{n}^{\prime \prime}=V_{n}+\sum_{k=2}^{n-1} e^{z_{n-1, k}} \epsilon_{\ell-k, \ell+1-k}
\end{aligned}
$$

при $n=3, \ldots, \ell$. Матрицы $U_{n}, \widetilde{U}_{n}$ и $V_{n}, \widetilde{V}_{n}$ удовлетворяют соотношениям

$$
\widetilde{U}_{n}=\widetilde{U}_{n}^{\prime \prime} U_{n}^{-1} \widetilde{U}_{n}^{\prime}, \quad \widetilde{V}_{n}=\widetilde{V}_{n}^{\prime} V_{n}^{-1} \widetilde{V}_{n}^{\prime \prime}, \quad n=3, \ldots, \ell
$$

Tеорема 1.13. і) Образ типичного элемента $v^{D_{\ell}} \in N_{+}^{(0)}$ в тавтологическом представлении $\pi: \mathfrak{s o}_{2 \ell} \rightarrow \operatorname{End}\left(\mathbb{C}^{2 \ell}\right)$ можно представить в виде

$$
\pi\left(v^{D_{\ell}}\right)=\mathfrak{X}_{2} \mathfrak{X}_{3} \cdots \mathfrak{X}_{\ell}, \quad \mathfrak{X}_{n}=\pi\left(\mathfrak{X}_{D_{n-1}}^{D_{n}}\right),
$$


где

$$
\begin{aligned}
& \mathfrak{X}_{2}= R_{\ell+1}\left[\widetilde{U}_{2} U_{2}^{-1}\right]^{*} R_{\ell+1} R_{\ell-1} \widetilde{U}_{2} U_{2}^{-1} R_{\ell-1} R_{\ell} R_{\ell-1} \\
& \times\left[\widetilde{V}_{2} V_{2}^{-1}\right]^{*} R_{\ell-1} R_{\ell+1} \widetilde{V}_{2} V_{2}^{-1} R_{\ell+1} R_{\ell}, \\
& \mathfrak{X}_{n}=\left[\widetilde{U}_{n}^{\prime \prime} U_{n}^{-1}\right]^{*} R_{\ell-1} \widetilde{U}_{n} U_{n}^{-1} R_{\ell-1} R_{\ell+1}\left[\widetilde{U}_{n}^{\prime} U_{n}^{-1}\right]^{*} R_{\ell+1} S_{\ell} R_{\ell-1} \\
& \quad \times\left[\widetilde{V}_{n}^{\prime} V_{n}^{-1}\right]^{*} R_{\ell-1} R_{\ell+1} \widetilde{V}_{n} V_{n}^{-1} R_{\ell+1} R_{\ell} \widetilde{V}_{n}^{\prime \prime} V_{n}^{-1}
\end{aligned}
$$

при $n=3, \ldots, \ell$ u $x_{\ell, i}=0, i=1, \ldots, \ell$, а элементы $R_{i}$ определяются следующим образом:

$$
R_{i}=\sum_{k=1}^{i-1} \epsilon_{k, k}+\epsilon_{i, i+1}+\epsilon_{i+1, i}+\sum_{k=i+2}^{2 \ell} \epsilon_{k, k} .
$$

ii) Формуль (1.213)-(1.216) определяют параметризацию множества полностью неотрицательных унипотентных элементов $N_{+}^{(0)} \subset N_{+}$.

ДокАЗАТЕЛьство. Пусть $v^{D_{\ell}}(y)$ - параметризация на $N_{+}$, заданная в (1.195), (1.196). Положим

$$
\begin{aligned}
& \widetilde{X}_{1}(y)=e^{y \epsilon_{\ell-1, \ell}}, \quad \widetilde{X}_{2}(y)=e^{2 y \epsilon_{\ell, \ell+2}}, \\
& \widetilde{X}_{k}(y)=e^{y \epsilon_{\ell+1-k, \ell+2-k}}, \quad k=3, \ldots, \ell
\end{aligned}
$$

Тогда действие инволюции * имеет вид

$$
\begin{gathered}
\tilde{X}_{1}(y)^{*}=e^{y \epsilon_{\ell+1, \ell+2}}, \quad \widetilde{X}_{2}(y)^{*}=e^{2 y \epsilon_{\ell-1, \ell+1}}, \\
\widetilde{X}_{k}(y)^{*}=\widetilde{X}_{\ell-1+k}(y), \quad k=3, \ldots, \ell
\end{gathered}
$$

Вложим элементарные унипотентные подгруппы $X_{i}(y)$ группы $\mathrm{SO}(2 \ell)$ в $\mathrm{GL}(2 \ell)$ следующим образом:

$$
X_{i}(y)=\tilde{X}_{i}(y)^{*} \tilde{X}_{i}(y), \quad i=1, \ldots, \ell .
$$

Это отображает произвольные регулярные унипотентные элемент $v^{D_{\ell}}$ в унипо-

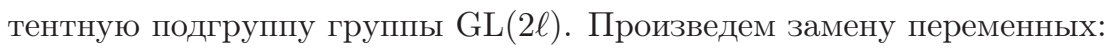

$$
\begin{aligned}
y_{1, n} & =e^{z_{n, 1}-x_{n, 1}}+e^{z_{n, 1}-x_{n+1,1}}, & & n=1, \ldots, \ell-1, \\
y_{2, n} & =e^{z_{n, 1}+x_{n, 1}}+e^{z_{n, 1}+x_{n+1,1}}, & & n=1, \ldots, \ell-1, \\
y_{k, 2 r-1} & =e^{z_{k+r-2, k-1}-x_{k+r-2, k-1}}, & & k=3, \ldots, \ell, \\
y_{k, 2 r} & =e^{z_{k+r-2, k-1}-x_{k+r-1, k-1}}, & & r=1, \ldots, \ell+1-k .
\end{aligned}
$$

Здесь $x_{\ell, k}=0$ при $k=1, \ldots, \ell$. Несложно проверить, что после замены $(1.217)$ образ $\pi\left(v^{D_{\ell}}\right)$ элемента $v^{D_{\ell}}$, определенный в $(1.195),(1.196)$, преобразуется в (1.213)-(1.215). Учитывая, что замена переменных (1.217) обратима, мы получаем параметризацию на $N_{+}^{(0)} \subset N_{+}$. Теорема 1.13 доказана. 
Модифицированная факторизационная параметризация на унипотентной группе $N_{+}$определяет специальную реализацию представления основной серии алгебры $\mathscr{U}\left(\mathfrak{s o}_{2 \ell}\right)$ дифференциальными операторами. Она может быть получена с использованием замены переменных (1.217) в реализации из предложения 1.12 .

Мы называем представлением Гаусса-Гивенталя эту реализацию представления алгебры $\mathscr{U}\left(\mathfrak{s o}_{2 \ell}\right)$.

ПРЕДЛОжЕНИЕ 1.13. Следующие дифференииальные операторь определяют представление $\pi_{\mu}$ алгебры $\mathscr{U}\left(\mathfrak{s o}_{2 \ell}\right)$ в пространстве $V_{\mu}$ в терминах модифицированной факторизационной параметризации на $N_{+}^{(0)}$ :

$$
\begin{gathered}
F_{i}=\sum_{n=1}^{\ell-1} e^{x_{\gamma_{i}(n), 1}-z_{n, 1}}\left[\frac{e^{x_{\gamma_{i}(n), 1}+x_{n+1,1}}-e^{x_{\gamma_{i *}(n), 1}}+e^{x_{n, 2}}}{\left(e^{x_{n, 1}}+e^{x_{n+1,1}}\right)^{2}} \frac{\partial}{\partial z_{n, 1}}+\left(\delta_{n, 1}-1\right) \frac{\partial}{\partial z_{n, 2}}\right. \\
\quad+\frac{\left(1-\delta_{n, 1}\right) e^{x_{n, 2}}+e^{x_{n+1,2}}}{e^{x_{n, 1}}+e^{x_{n+1,1}}} \sum_{k=1}^{n}\left\{(-1)^{\ell+i^{*}-k} \frac{\partial}{\partial x_{k, 1}}-\delta_{k, n} \frac{\partial}{\partial x_{k, 2}}\right. \\
\left.\left.\quad+(-1)^{\ell+i-k} \frac{e^{x_{k, 1}}-e^{x_{k+1,1}}}{e^{x_{k, 1}}+e^{x_{k+1,1}}} \frac{\partial}{\partial z_{k, 1}}\right\}\right], \quad i=1,2,
\end{gathered}
$$

где *: $1 \leftrightarrow 2, \varpi(n)=\left(1-(-1)^{n}\right) / 2$ - четность числа $n$ и $\gamma_{i}(n)=2[(n+\varpi(\ell+$ $1-i)) / 2]+\varpi(\ell-i)$

$$
\begin{aligned}
F_{k}= & \sum_{n=1}^{2(\ell-k)+1} e^{x_{\beta(n)+k-1, k}-z_{\alpha(n)+k-1, k-1}}\left[\frac{\partial}{\partial x_{\alpha(n)+k-1, k-1}}+\left(\delta_{n, 1}-1\right) \frac{\partial}{\partial x_{\alpha(n)+k-1, k}}\right. \\
& \left.+\varpi(n)\left\{\frac{\partial}{\partial z_{\alpha(n)+k-1, k-1}}+\left(\delta_{n, 1}-1\right) \frac{\partial}{\partial z_{\alpha(n)+k-1, k}}\right\}\right]
\end{aligned}
$$

npu $k=3, \ldots, \ell u$

$$
\begin{gathered}
H_{i}=\left\langle\mu, \alpha_{i}^{\vee}\right\rangle+2\left\{\sum_{n=1}^{\ell-1} \frac{\varpi\left(n+i^{*}\right) e^{x_{n, 1}}+\varpi(n+i) e^{x_{n+1,1}}}{e^{x_{n, 1}}+e^{x_{n+1,1}}}\right. \\
\left.+(-1)^{i}\left(\frac{\partial}{\partial x_{1,1}}+\frac{\partial}{\partial x_{\ell-1,1}}\right)\right\}-\sum_{n=2}^{\ell-1} \frac{\partial}{\partial z_{n, 2}}
\end{gathered}
$$

nрu $i=1,2 u \varpi(k)=\left(1-(-1)^{k}\right) / 2$,

$$
\begin{aligned}
H_{k}= & \left\langle\mu, \alpha_{k}^{\vee}\right\rangle+\sum_{i=2}^{\ell} a_{k, i} \sum_{j=i}^{\ell} \frac{\partial}{\partial z_{j-1, i-1}}, \quad k=3, \ldots, \ell, \\
E_{i}= & \sum_{n=1}^{2 \ell-2} e^{z_{\beta(n), 1}+(-1)^{i} x_{\alpha(n)+1,1}\left[\left\langle\mu, \alpha_{i}^{\vee}\right\rangle+(-1)^{i+1}\right.} \\
& \times \sum_{k=1}^{\beta(n)}\left\{\frac{\partial}{\partial x_{k, 1}}+\left(1-\delta_{k, 1}\right) \delta_{k, \beta(n)} \frac{\partial}{\partial x_{k, 2}}\right\}
\end{aligned}
$$




$$
\left.-\sum_{k=1}^{\alpha(n)}\left\{\frac{\partial}{\partial z_{k, 1}}+\delta_{k, 1}\left(1-\delta_{k, \alpha(n)}\right) \frac{\partial}{\partial z_{k, 2}}\right\}\right]
$$

npu $i=1,2 u$

$$
\begin{aligned}
E_{k}= & \sum_{n=1}^{2(\ell+1-k)} e^{z_{\beta(n)+k-2, k-2}-x_{\alpha(n)+k-1, k-1}}\left[\left\langle\mu, \alpha_{k}^{\vee}\right\rangle+\frac{\partial}{\partial z_{k-2, k-2}}\right. \\
& +\frac{\partial}{\partial x_{\beta(n)+k-2, k-1}}+\left(\delta_{\beta(n), 1}-1\right) \frac{\partial}{\partial x_{\beta(n)+k-2, k}} \\
& +\sum_{m=1}^{\alpha(n)}\left\{\frac{\partial}{\partial z_{k-2+m, k-2}}-2 \frac{\partial}{\partial z_{k-2+m, k-1}}+\left(1-\delta_{m, 1}\right) \frac{\partial}{\partial z_{k-2+m, k}}\right\} \\
& \left.+(1-\varpi(n))\left\{\frac{\partial}{\partial z_{\alpha(n)+k-2, k-1}}+\left(\delta_{n, 2}-1\right) \frac{\partial}{\partial z_{\alpha(n)+k-2, k}}\right\}\right],
\end{aligned}
$$

где $3 \leqslant k \leqslant \ell u x_{\ell, k}=0$.

Мы собираемся явно выписать матричный элемент (1.12) при $\mathfrak{g}=\mathfrak{s o}_{2 \ell}$, используя определенное выше представление Гаусса-Гивенталя. Векторы Уиттекера $\psi_{R}$ и $\psi_{L}$ в этом представлении должны удовлетворять системе дифференциальных уравнений (1.7) и (1.8). Ее решения имеют следующий вид.

ЛЕмма 1.10. Имеют место следующие выражения для левого и правого векторов Уиттекера:

$$
\begin{aligned}
& \psi_{R}=\exp \{-\sum_{n=1}^{\ell-1}\left(e^{z_{n, 1}-x_{n, 1}}+e^{z_{n, 1}-x_{n+1,1}}+e^{z_{n, 1}+x_{n, 1}}+e^{z_{n, 1}+x_{n+1,1}}\right) \\
&\left.-\sum_{k=3}^{\ell} \sum_{n=1}^{\ell+1-k}\left(e^{z_{k+n-2, k-1}-x_{k+n-2, k-1}}+e^{z_{k+n-2, k-1}-x_{k+n-1, k-1}}\right)\right\}, \quad(1.2 \\
& \psi_{L}=e^{2 \mu_{1} x_{1,1}} \prod_{n=2}^{\ell}\left(e^{x_{n, 1}}+e^{x_{n-1,1}}\right)^{2 \mu_{n}} \\
& \quad \times \prod_{n=1}^{\ell} \exp \left\{-\mu_{n}\left(\sum_{i=1}^{n} x_{n, i}-2 \sum_{i=1}^{n-1} z_{n-1, i}+\sum_{i=1}^{n-1} x_{n-1, i}\right)\right\} \\
& \quad \times \exp \left\{-\sum_{k=1}^{\ell-1}\left(e^{x_{k+1, k+1}-z_{k, k}}+\sum_{i=k+1}^{\ell-1}\left(e^{x_{i, k+1}-z_{i, k}}+e^{x_{i+1, k+1}-z_{i, k}}\right)\right)\right\}
\end{aligned}
$$

где $x_{\ell, k}=0, k=1, \ldots, \ell, \mu_{n}=\imath \lambda_{n}-\rho_{n}, \rho_{1}=0$ u $\rho_{n}=n-1$ nрu $1<n \leqslant \ell$ $\left(\sum_{i}^{j}=0\right.$ npu $\left.j<i\right)$.

Теперь мы готовы указать интегральное представление спаривания (1.12) при $\mathfrak{g}=\mathfrak{s o}_{2 \ell}$. Чтобы получить явное выражение для подынтегральной функции, мы используем тот же тип разложения элемента максимального тора, что 
и для других классических групп в предыдущих разделах:

$$
e^{-H_{x}}=\pi_{\lambda}\left(\exp \left(-\sum_{i=1}^{\ell}\left\langle\omega_{i}, x\right\rangle h_{i}\right)\right)=e^{H_{L}} e^{H_{R}}
$$

где

$$
\begin{aligned}
-H_{x}=H_{L} & +H_{R}=\sum_{i=1}^{\ell} \mu_{i} x_{\ell, i}+\sum_{k=3}^{\ell}\left(x_{\ell, k}-x_{\ell, k-1}\right) \sum_{i=k-1}^{\ell-1} \frac{\partial}{\partial z_{i, k-1}}+x_{\ell, 2} \sum_{i=1}^{\ell-1} \frac{\partial}{\partial z_{i, 1}} \\
& +x_{\ell, 1}\left(\frac{\partial}{\partial x_{\ell-1,1}}+\frac{\partial}{\partial x_{1,1}}-\sum_{k=1}^{\ell-1}(-1)^{k} \frac{e^{x_{k+1,1}}-e^{x_{k, 1}}}{e^{x_{k, 1}}+e^{x_{k+1,1}}} \frac{\partial}{\partial z_{k, 1}}\right)
\end{aligned}
$$

и

$$
\begin{gathered}
H_{L}=\sum_{k=1}^{\ell} x_{\ell, k}\left(\sum_{i=k}^{\ell-1} \frac{\partial}{\partial x_{i, k}}+\sum_{i=k-1}^{\ell-1} \frac{\partial}{\partial z_{i, k-1}}\right) \\
H_{R}=-H_{x}-H_{L} .
\end{gathered}
$$

Мы считаем, что $H_{L}$ действует на левый вектор и $H_{R}$ действует на правый вектор в (1.12). Принимая во внимание предложение 1.10, мы приходим к следующей теореме.

Теорема 1.14. Собственные функиии $\mathfrak{s o}_{2 \ell}$-цепочки Тоды (1.12) допускают следующее интегральное представление:

$$
\Psi_{\lambda_{1}, \ldots, \lambda_{\ell}}^{D_{\ell}}\left(x_{\ell, 1}, \ldots, x_{\ell, \ell}\right)=\int_{C} \prod_{k=1}^{\ell-1} d \underline{x}_{k} d \underline{z}_{k} e^{\mathscr{F}^{D_{\ell}}}
$$

где

$$
\begin{aligned}
\mathscr{F}^{D_{\ell}}=\imath \lambda_{1} & x_{1,1}-\sum_{n=2}^{\ell} \imath \lambda_{n}\left(\sum_{i=1}^{n} x_{n, i}-2 \sum_{i=1}^{n-1} z_{n-1, i}+\sum_{i=1}^{n-1} x_{n-1, i}-2 \ln \left(e^{x_{n, 1}}+e^{x_{n-1,1}}\right)\right) \\
& -\sum_{k=1}^{\ell-1}\left(e^{x_{k+1, k+1}-z_{k, k}}+\sum_{i=k+1}^{\ell-1}\left(e^{x_{i, k+1}-z_{i, k}}+e^{x_{i+1, k+1}-z_{i, k}}\right)\right) \\
& -\sum_{n=1}^{\ell-1}\left(e^{z_{n, 1}-x_{n, 1}}+e^{z_{n, 1}-x_{n+1,1}}+e^{z_{n, 1}+x_{n, 1}}+e^{z_{n, 1}+x_{n+1,1}}\right) \\
& -\sum_{k=3}^{\ell} \sum_{n=1}^{\ell+1-k}\left(e^{z_{k+n-2, k-1}-x_{k+n-2, k-1}}+e^{z_{k+n-2, k-1}-x_{k+n-1, k-1}}\right),
\end{aligned}
$$

и $x_{i}:=x_{\ell, i}, 1 \leqslant i \leqslant \ell$, a $C \subset N_{+}$- некомпактное подмногообразие половинной размерности, для которого подынтегральная функиия экспоненииально убывает на границе и на бесконечности. В частности, в качестве $C$ можно взять малую деформацию подпространства $\mathbb{R}^{\ell(\ell-1)} \subset \mathbb{C}^{\ell(\ell-1)}$, обеспечивающую сходимость интеграла. 
Заметим, что интеграл (1.229) можно получить непосредственно из (1.205), используя замену переменных:

$$
\begin{aligned}
y_{1, n} & =e^{x_{\ell, 1}-x_{\ell, 2}}\left(e^{z_{n, 1}-x_{n, 1}}+e^{z_{n, 1}-x_{n+1,1}}\right), & & n=1, \ldots, \ell-1, \\
y_{2, n} & =e^{-x_{\ell, 1}-x_{\ell, 2}} e^{z_{n, 1}+x_{n, 1}}+e^{z_{n, 1}+x_{n+1,1}}, & & n=1, \ldots, \ell-1, \\
y_{k, 2 r-1} & =e^{x_{\ell, k-1}-x_{\ell, k}} e^{z_{k+r-2, k-1}-x_{k+r-2, k-1}}, & & k=3, \ldots, \ell, \\
y_{k, 2 r} & =e^{x_{\ell, k-1}-x_{\ell, k}} e^{z_{k+r-2, k-1}-x_{k+r-1, k-1}}, & & r=1, \ldots, \ell+1-k .
\end{aligned}
$$

ПримеР 1.7. При $\ell=2$ общая формула (1.229) принимает вид

$$
\begin{aligned}
& \Psi_{\lambda_{1}, \lambda_{2}}^{D_{2}}\left(x_{2,1}, x_{2,2}\right)=\int_{C} d x_{1,1} d z_{1,1}\left(e^{x_{1,1}}+e^{x_{2,1}}\right)^{2 \imath \lambda_{2}} \\
& \quad \times \exp \left\{\imath \lambda_{1} x_{1,1}-\imath \lambda_{2}\left(x_{2,1}+x_{2,2}-2 z_{1,1}+x_{1,1}\right)\right. \\
& \left.\quad-\left(e^{z_{1,1}-x_{1,1}}+e^{z_{1,1}+x_{1,1}}+e^{z_{1,1}-x_{2,1}}+e^{z_{1,1}+x_{2,1}}+e^{x_{2,2}-z_{1,1}}\right)\right\}
\end{aligned}
$$

Рассмотрим преобразование переменных интегрирования

$$
z_{1,1} \longmapsto-z_{1,1}+x_{2,2}-\ln \left(e^{x_{1,1}}+e^{x_{2,1}}\right), \quad x_{1,1} \longmapsto x_{1,1}-z_{1,1}-x_{2,1},
$$

которое влечет

$$
\begin{aligned}
& \Psi_{\lambda_{1}, \lambda_{2}}^{D_{2}}\left(x_{2,1}, x_{2,2}\right)=e^{-\imath \lambda_{1} x_{2,1}+\imath \lambda_{2} x_{2,2}} \int_{C} d z_{1,1} d x_{1,1} \exp \left\{\imath\left(\lambda_{1}-\lambda_{2}\right) x_{1,1}\right. \\
& \left.\quad-\imath\left(\lambda_{1}+\lambda_{2}\right) z_{1,1}-\left(e^{z_{1,1}+x_{2,1}}+e^{x_{2,2}-z_{1,1}}\right)-\left(e^{x_{2,2}-x_{1,1}}+e^{x_{1,1}-x_{2,1}}\right)\right\} .
\end{aligned}
$$

Теперь сделаем следующую замену переменных интегрирования:

$$
z_{1,1} \longmapsto z_{1,1}+\frac{x_{2,2}-x_{2,1}}{2}, \quad x_{1,1} \longmapsto x_{1,1}+\frac{x_{2,1}+x_{2,2}}{2},
$$

и получим

$$
\Psi_{\lambda_{1}, \lambda_{2}}^{D_{2}}\left(x_{2,1}, x_{2,2}\right)=K_{\imath\left(\lambda_{1}-\lambda_{2}\right)}\left(2 e^{\frac{x_{2,2}-x_{2,1}}{2}}\right) K_{-\imath\left(\lambda_{1}+\lambda_{2}\right)}\left(2 e^{\frac{x_{2,1}+x_{2,2}}{2}}\right),
$$

где с учетом (1.50)

$$
K_{\imath \gamma}\left(2 e^{y}\right)=\int_{\mathbb{R}} d t e^{\imath \gamma t-e^{y}\left(e^{t}+e^{-t}\right)}=\Psi_{\gamma}^{\mathfrak{s l} 2}(y) .
$$

Отождествление функций Уиттекера (1.233) обеспечивается изоморфизмом алгебр Ли $\mathfrak{s o}_{4} \simeq \mathfrak{s l}_{2} \oplus \mathfrak{s l}_{2}$.

Имеется простое комбинаторное описание потенциала $\mathscr{F}^{D_{\ell}}$ на нулевом уровне спектра $\left\{\lambda_{i}=0\right\}$. А именно, его можно представить в виде суммы по всем 
стрелкам следующей диаграммы:

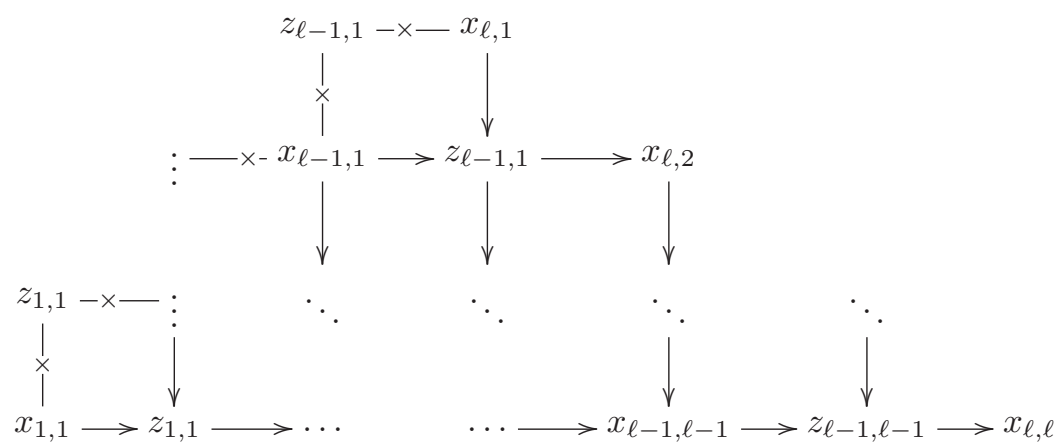

Мы используем то же правило сопоставления переменных стрелкам диаграммы, что и для случая $A_{\ell}$. В дополнение мы сопоставляем символу $z-\times-x$ экспоненту $e^{z+x}$.

Заметим, что диаграмма для $D_{\ell}$ может быть получена посредством факторизации из диаграммы $A_{2 \ell-1}$. Рассмотрим инволюцию

$$
*: X \longmapsto-\dot{W}_{0}^{-1} X^{t} \dot{W}_{0}
$$

где $\dot{W}_{0}$ - подъем элемента максимальной длины группы Вейля $S_{2 \ell}$ и $X^{t}$ обозначает стандартное транспонирование. Соответствующее действие на модифицированных параметрах факторизации имеет вид

$$
*: x_{k, i} \longleftrightarrow-x_{k, k+1-i}
$$

Это определяет факторизацию $A_{2 \ell-1}$-диаграммы, которая приводит к диаграмме для $D_{\ell}$. Заметим, что диаграмму для $D_{\ell}$ также можно получить, стирая последний ряд вершин и стрелок на правом склоне диаграммы для $C_{\ell}$.

Имеется следующий аналог соотношений (1.52). Введем переменные $a_{i, k}$, $b_{i, k}, c_{i, k}, d_{i, k}$, связанные со стрелками диаграммы:

$$
\begin{gathered}
a_{n, 1}=e^{x_{n, 1}+z_{n-1,1}}, \quad a_{n, i}=e^{x_{n, i}-z_{n-1, i-1}}, \quad 2 \leqslant i \leqslant n \leqslant \ell ; \\
b_{k, 1}=e^{x_{k, 1}+z_{k, 1}}, \quad k=1, \ldots, \ell-1 ; \\
b_{n, i}=e^{z_{n, i}-x_{n, i-1}}, \quad 2 \leqslant i \leqslant n<\ell ; \\
c_{m, j}=e^{z_{m, j}-x_{m, j}}, \quad d_{m, j}=e^{z_{m, j}-x_{m+1, j}}, \quad 1 \leqslant j \leqslant m<\ell .
\end{gathered}
$$

Тогда выполнены соотношения

$$
\begin{gathered}
b_{k, i} c_{k, i}=a_{k+1, i} d_{k, i}, \quad a_{k+1, i+1} d_{k, i}=b_{k+1, i+1} c_{k+1, i}, \quad 1 \leqslant i \leqslant k<\ell, \\
a_{\ell, 1} a_{\ell, 2}=e^{x_{\ell, 1}+x_{\ell, 2}}, \quad a_{\ell, i} d_{\ell-1, i-1}=e^{x_{\ell, i}-x_{\ell, i-1}}, \quad i=2, \ldots, \ell .
\end{gathered}
$$


1.5.3. Рекурсия для $\mathfrak{s o}_{2 \ell}$-функций Уиттекера и $\mathscr{Q}$-оператор для $D_{\ell}^{(1)}$-цепочки Тоды. Интегральное представление (1.229) для $\mathfrak{s o}_{2 \ell}$-функций Уиттекера имеет рекурсивную структуру относительно ранга $\ell$. Для всех $n=2, \ldots, \ell$ рассмотрим интегральные операторы $Q_{D_{n-1}}^{D_{n}}$ с ядрами $Q_{D_{n-1}}^{D_{n}}\left(\underline{x}_{n}, \underline{x}_{n-1} ; \lambda_{n}\right)$, которые определяются следующим образом:

$$
\begin{aligned}
& Q_{D_{n-1}}^{D_{n}}\left(\underline{x}_{n}, \underline{x}_{n-1} ; \lambda_{n}\right)=\int d \underline{z}_{n} Q_{C_{n-1}}^{D_{n}}\left(\underline{x}_{n}, \underline{z}_{n-1}\right) Q_{D_{n-1}}^{C_{n-1}}\left(\underline{z}_{n-1}, \underline{x}_{n-1}\right) \\
& \quad \times \exp \left\{-\imath \lambda_{n}\left(\sum_{i=1}^{n} x_{n, i}-2 \sum_{i=1}^{n-1} z_{n-1, i}+\sum_{i=1}^{n-1} x_{n-1, i}\right)\right\}\left(e^{x_{n-1,1}}+e^{x_{n, 1}}\right)^{2 \imath \lambda_{n}}
\end{aligned}
$$

где

$$
\begin{aligned}
& Q_{C_{n-1}}^{D_{n}}\left(\underline{x}_{n}, \underline{z}_{n-1}\right)=\exp \left\{-\left(e^{x_{n, 1}+z_{n-1,1}}+\sum_{i=1}^{n-1}\left(e^{z_{n-1, i}-x_{n, i}}+e^{x_{n, i+1}-z_{n-1, i}}\right)\right)\right\} \\
& Q_{D_{n-1}}^{C_{n-1}}\left(\underline{z}_{n-1}, \underline{x}_{n-1}\right)=\exp \left\{-\left(e^{x_{n-1,1}+z_{n-1,1}}\right.\right. \\
& \left.\left.\quad+\sum_{i=1}^{n-2}\left(e^{z_{n-1, i}-x_{n-1, i}}+e^{x_{n-1, i+1}-z_{n-1, i}}\right)+e^{z_{n-1, n-1}-x_{n-1, n-1}}\right)\right\}
\end{aligned}
$$

и при $n=1$ мы полагаем

$$
Q_{D_{0}}^{D_{1}}\left(x_{1,1} ; \lambda_{1}\right)=e^{\imath \lambda_{1} x_{1,1}}
$$

С использованием $Q_{D_{n-1}}^{D_{n}}, n=1, \ldots, \ell$, интегральное представление $(1.229)$ можно записать в рекурсивной форме.

Теорема 1.15. Собственные функции $\mathfrak{s o}_{2 \ell}$-цепочки Тоды можно записать в следующей рекурсивной форме:

$$
\Psi_{\lambda_{1}, \ldots, \lambda_{\ell}}^{D_{\ell}}\left(x_{1}, \ldots, x_{\ell}\right)=\int_{C} \prod_{k=1}^{\ell-1} d \underline{x}_{k} \prod_{k=1}^{\ell} Q_{D_{k}-1}^{D_{k}}\left(\underline{x}_{k}, \underline{x}_{k-1} ; \lambda_{k}\right),
$$

или, эквивалентно,

$$
\Psi_{\lambda_{1}, \ldots, \lambda_{\ell}}^{D_{\ell}}\left(\underline{x}_{\ell}\right)=\int_{\mathscr{C}_{\ell}} d \underline{x}_{\ell-1} Q_{D_{\ell-1}}^{D_{\ell}}\left(\underline{x}_{\ell}, \underline{x}_{\ell-1} ; \lambda_{\ell}\right) \Psi_{\lambda_{1}, \ldots, \lambda_{\ell-1}}^{D_{\ell-1}}\left(\underline{x}_{\ell-1}\right),
$$

где $x_{n}:=x_{\ell, n}, 1 \leqslant n \leqslant \ell$. Здесъ $C \subset N_{+}-$некомпактное подмногообразие половинной размерности, для которого подынтегральная функция экспоненциально убывает на границе и на бесконечности.

Как и для других классических групп, отличных от $\mathfrak{g l}_{\ell+1}$, специализация на нулевом уровне спектра $\left\{\lambda_{n}=0\right\}$ обнаруживает более тонкую рекурсивную структуру. В этом случае ядро оператора $Q_{D_{n-1}}^{D_{n}}$ редуцируется к свертке двух ядер $Q_{C_{n-1}}^{D_{n}}\left(\underline{x}_{n}, \underline{z}_{n-1}\right)$ и $Q_{D_{n-1}}^{C_{n-1}}\left(\underline{z}_{n-1}, \underline{x}_{n-1}\right)$. Соответствующие интегральные операторы $Q_{C_{n-1}}^{D_{n}}, Q_{D_{n-1}}^{C_{n-1}}$ можно рассматривать как элементарные сплетающие операторы, связывающие гамильтонианы цепочек Тоды для систем корней $D_{n}, C_{n-1}$ и $C_{n-1}, D_{n-1}$. Тем самым, несложно непосредственно проверить следующие соотношения для квадратичных гамильтонианов. 
Лемма 1.11. Операторъ $Q_{D_{n-1}}^{D_{n}}, Q_{C_{n-1}}^{D_{n}} u Q_{D_{n-1}}^{C_{n-1}}$ удовлетворяют следующим сплетающим соотношениям с квадратичными гамильтонианами Тоды.

1. Операторы $Q_{C_{n-1}^{D_{n}}}^{D_{n}} Q_{D_{n-1}}^{C_{n-1}}$ сплетают квадратичные гамильтонианы $C$ и D-цепочек Tодь:

$$
\begin{aligned}
\mathscr{H}_{2}^{D_{n}}\left(\underline{x}_{n}\right) Q_{C_{n-1}}^{D_{n}}\left(\underline{x}_{n}, \underline{z}_{n-1}\right) & =Q_{C_{n-1}}^{D_{n}}\left(\underline{x}_{n}, \underline{z}_{n-1}\right) \mathscr{H}_{2}^{C_{n-1}}\left(\underline{x}_{n-1}\right), \\
\mathscr{H}_{2}^{C_{n}}\left(\underline{z}_{n}\right) Q_{D_{n}}^{C_{n}}\left(\underline{z}_{n}, \underline{x}_{n}\right) & =Q_{D_{n}}^{C_{n}}\left(\underline{z}_{n}, \underline{x}_{n}\right) \mathscr{H}_{2}^{D_{n}}\left(\underline{x}_{n}\right) .
\end{aligned}
$$

2. Onератор $Q_{D_{n-1}}^{D_{n}}$ nри $\lambda_{n}=0$ cплетает гамильтониань $\mathscr{H}_{2}^{D_{n}}$ и $\mathscr{H}_{2}^{D_{n-1}}$ :

$$
\mathscr{H}_{2}^{D_{n}}\left(\underline{x}_{n}\right) Q_{D_{n-1}}^{D_{n}}\left(\underline{x}_{n}, \underline{x}_{n-1} ; \lambda_{n}=0\right)=Q_{D_{n-1}}^{D_{n}}\left(\underline{x}_{n}, \underline{x}_{n-1} ; \lambda_{n}=0\right) \mathscr{H}_{2}^{D_{n-1}}\left(\underline{x}_{n-1}\right),
$$

әде

$$
\begin{aligned}
& \mathscr{H}_{2}^{C_{n}}=-\frac{1}{2} \sum_{i=1}^{n} \frac{\partial^{2}}{\partial z_{i}^{2}}+2 e^{2 z_{1}}+\sum_{i=1}^{n-2} e^{z_{i+1}-z_{i}} \\
& \mathscr{H}_{2}^{D_{n}}=-\frac{1}{2} \sum_{i=1}^{n} \frac{\partial^{2}}{\partial x_{i}^{2}}+e^{x_{1}+x_{2}}+\sum_{i=1}^{n-1} e^{x_{i+1}-x_{i}}
\end{aligned}
$$

Интегральное ядро оператора $Q_{D_{n-1}}^{D_{n}}\left(\underline{x}_{n}, \underline{x}_{n-1}\right)$ при $\lambda_{n}=0$ задается следующей диаграммой:

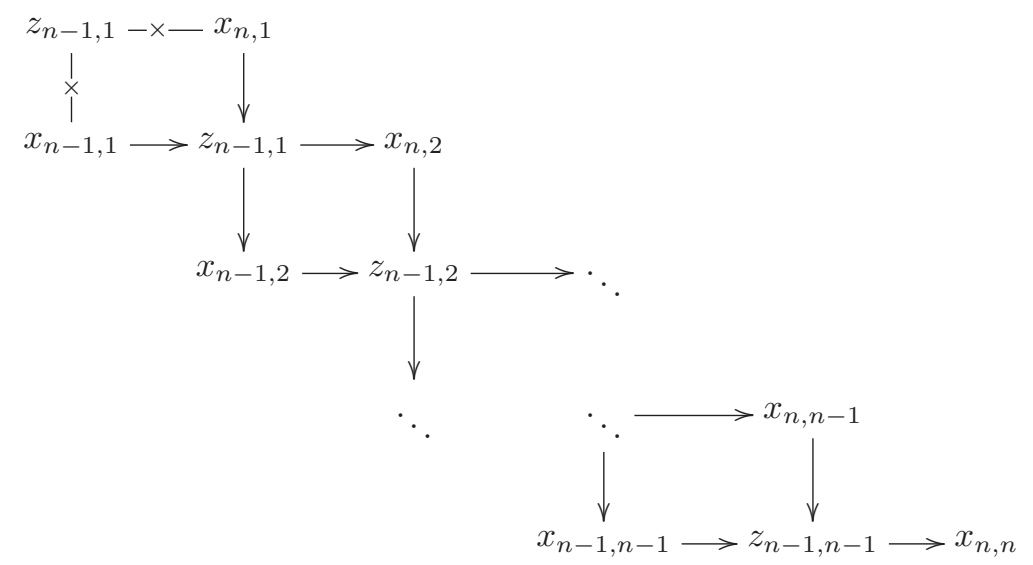

Здесь нижние и верхние спускающиеся пути ориентированной диаграммы отвечают ядрам элементарных сплетений $Q_{C_{n-1}^{D_{n}}}^{D_{n}} Q_{D_{n-1}}^{C_{n}}$ соответственно. Свертка ядер $Q_{C_{n}}^{D_{n}}$ и $Q_{D_{n-1}}^{C_{n-1}}$ соответствует интегрированию по переменным $z_{n-1, i}$ на внутренних вершинах поддиаграммы (1.245).

По аналогии с классическими алгебрами Ли других серий, операторы рекурсии $Q_{D_{n-1}}^{D_{n}}$ можно рассматривать как вырождения $\mathscr{Q}$-операторов Бакстера для аффинных $D_{\ell}^{(1)}$-цепочек Тоды. Напомним корневые данные для $D_{\ell}^{(1)}$. Простые корни для аффинной системы корней $D_{\ell}^{(1)}$ можно задать как векторы в $\mathbb{R}^{\ell}$ следующим образом:

$$
\alpha_{1}=\epsilon_{1}+\epsilon_{2}, \quad \alpha_{i+1}=\epsilon_{i+1}-\epsilon_{i}, \quad 1 \leqslant i \leqslant \ell-1, \quad \alpha_{\ell+1}=-\epsilon_{\ell}-\epsilon_{\ell-1} .
$$


Диаграмма Дынкина здесь имеет вид

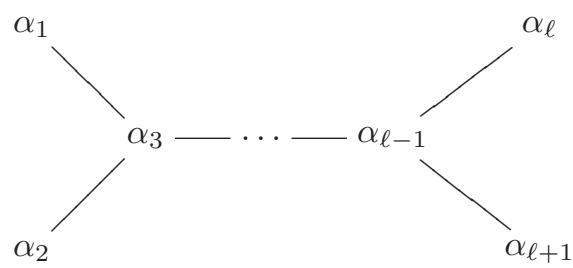

Соответствующий квадратичный гамильтониан $D_{\ell}^{(1)}$-цепочки Тоды определяется как

$$
\mathscr{H}_{2}^{D_{\ell}^{(1)}}=-\frac{1}{2} \sum_{i=1}^{\ell} \frac{\partial^{2}}{\partial x_{i}^{2}}+e^{x_{1}+x_{2}}+\sum_{i=1}^{\ell-2} e^{x_{i+1}-x_{i}}+g e^{x_{\ell}-x_{\ell-1}}+g e^{-x_{\ell}-x_{\ell-1}} .
$$

Определим $\mathscr{Q}$-оператор Бакстера для $D_{\ell}^{(1)}$-цепочки Тоды как интегральный оператор со следующим интегральным ядром:

$$
\begin{aligned}
& \mathscr{Q}^{D_{\ell}^{(1)}}\left(\underline{x}^{(\ell)}, \underline{y}^{(\ell)} ; \lambda\right)=\int d \underline{z}^{(\ell-1)} Q_{C_{\ell-1}^{(1)}}^{D_{\ell}^{(1)}}\left(\underline{x}^{(\ell)}, \underline{z}^{(\ell-1)}\right) Q_{D_{\ell}^{(1)}}^{C_{\ell-1}^{(1)}}\left(\underline{z}^{(\ell-1)}, \underline{y}^{(\ell)}\right) \\
& \quad \times \exp \left\{-\imath \lambda\left(\sum_{i=1}^{\ell} x_{i}-2 \sum_{i=1}^{\ell-1} z_{i}+\sum_{i=1}^{\ell} y_{i}\right)\right\}\left(e^{x_{1}}+e^{y_{1}}\right)^{2 \imath \lambda}\left(e^{-x_{\ell}}+e^{-y_{\ell}}\right)^{-2 \imath \lambda}
\end{aligned}
$$

где

$$
Q_{C_{\ell-1}^{(1)}}^{D_{\ell}^{(1)}}\left(\underline{x}^{(\ell)}, \underline{z}^{(\ell-1)}\right)=\exp \left\{-e^{z_{1}+x_{1}}-\sum_{i=1}^{\ell-1}\left(e^{z_{i}-x_{i}}+e^{x_{i+1}-z_{i}}\right)-g e^{-x_{\ell}-z_{\ell-1}}\right\}
$$

и

$$
Q_{C_{\ell-1}^{(1)}}^{D_{\ell}^{(1)}}\left(x_{1}, \ldots, x_{\ell} ; z_{1}, \ldots, z_{\ell}\right)=Q_{D_{\ell}^{(1)}}^{C_{\ell-1}^{(1)}}\left(z_{1}, \ldots, z_{\ell} ; x_{1}, \ldots, x_{\ell}\right) .
$$

Здесь мы используем обозначения $\underline{x}^{(\ell)}=\left(x_{1}, \ldots, x_{\ell}\right)$ и $\underline{y}^{(\ell)}=\left(y_{1}, \ldots, y_{\ell}\right)$.

ПредлоЖениЕ 1.14. Q-оператор (1.247) коммутирует с квадратичным гамильтонианом $D_{\ell}^{(1)}$-иепочки Тодъ:

$$
\mathscr{H}^{D_{\ell}^{(1)}}\left(\underline{x}^{(\ell)}\right) \mathscr{Q}^{D_{\ell}^{(1)}}\left(\underline{x}^{(\ell)}, \underline{y}^{(\ell)}\right)=\mathscr{Q}^{D_{\ell}^{(1)}}\left(\underline{x}^{(\ell)}, \underline{y}^{(\ell)}\right) \mathscr{H}^{D_{\ell}^{(1)}}\left(\underline{y}^{(\ell)}\right) .
$$

Покажем, что оператор рекурсии $Q_{D_{\ell-1}}^{D_{\ell}}$ можно рассматривать как обобщение $\mathscr{Q}$-операторов Бакстера для $D_{\ell}^{(1)}$. Введем слегка модифицированный оператор рекурсии с ядром $Q_{D_{\ell-1} \oplus D_{1}}^{D_{\ell}}$ :

$$
Q_{D_{\ell-1} \oplus D_{1}}^{D_{\ell}}\left(\underline{x}^{(\ell)}, \underline{y}^{(\ell)} ; \lambda\right)=e^{\imath \lambda y_{\ell}} Q_{D_{\ell-1}}^{D_{\ell}}\left(\underline{x}^{(\ell)}, \underline{y}^{(\ell-1)} ; \lambda\right),
$$

где $\underline{y}^{(\ell-1)}=\left(y_{1}, \ldots, y_{\ell-1}\right)$. Этот оператор сплетает гамильтонианы $\mathfrak{s o}_{2 \ell^{-}}$и $\mathfrak{s o}_{2 \ell-2} \oplus \mathfrak{s o}_{2}$-цепочек Тоды. Например, для квадратичных гамильтонианов получаем

$$
\begin{aligned}
\mathscr{H}_{2}^{D_{\ell}} & \left(\underline{x}^{(\ell)}\right) Q_{D_{\ell-1} \oplus D_{1}}^{D_{\ell}}\left(\underline{x}^{(\ell)}, \underline{y}^{(\ell)} ; \lambda\right) \\
& =Q_{D_{\ell-1} \oplus D_{1}}^{D_{\ell}}\left(\underline{x}^{(\ell)}, \underline{y}^{(\ell)} ; \lambda\right)\left(\mathscr{H}_{2}^{D_{\ell-1}}\left(\underline{y}^{(\ell-1)}\right)+\mathscr{H}_{2}^{D_{1}}\left(y_{\ell}\right)\right),
\end{aligned}
$$


где $\mathscr{H}_{2}^{D_{1}}\left(y_{\ell}\right)=-\frac{1}{2} \frac{\partial^{2}}{\partial y_{\ell}^{2}}$. Очевидно, что проекция соотношения на подпространство функций $F\left(\underline{y}^{(\ell)}\right)=\exp \left(\imath \lambda y_{\ell}\right) f\left(\underline{y}^{(\ell-1)}\right)$ приводит к исходному оператору рекурсии, для которого

$$
\begin{aligned}
\mathscr{H}_{2}^{D_{\ell}} & \left(\underline{x}^{(\ell)}\right) Q_{D_{\ell-1}}^{D_{\ell}}\left(\underline{x}^{(\ell)}, \underline{y}^{(\ell-1)} ; \lambda\right) \\
& =Q_{D_{\ell-1}}^{D_{\ell}}\left(\underline{x}^{(\ell)}, \underline{y}^{(\ell-1)} ; \lambda\right)\left(\mathscr{H}_{2}^{D_{\ell-1}}\left(\underline{y}^{(\ell-1)}\right)+\frac{1}{2} \lambda^{2}\right) .
\end{aligned}
$$

Введем однопараметрическое семейство операторов с ядрами

$$
\begin{aligned}
\mathscr{Q}^{D_{\ell}^{(1)}} & \left(\underline{x}^{(\ell)}, \underline{y}^{(\ell)} ; \lambda ; \varepsilon\right):=\varepsilon^{\imath \lambda} e^{\imath \lambda y_{\ell}} \int d \underline{z}^{(\ell-1)} Q_{C_{\ell-1}^{(1)}}^{D_{\ell}^{(1)}}\left(\underline{x}^{(\ell)}, \underline{z}^{(\ell-1)}\right) Q_{D_{\ell}^{(1)}}^{C_{\ell-1}^{(1)}}\left(\underline{z}^{(\ell-1)}, \underline{y}^{(\ell)} ; \varepsilon\right) \\
& \times \exp \left\{-\imath \lambda\left(\sum_{i=1}^{\ell} x_{i}-2 \sum_{i=1}^{\ell-1} z_{i}+\sum_{i=1}^{\ell-1} y_{i}\right)\right\}\left(e^{x_{1}}+e^{y_{1}}\right)^{2 \imath \lambda}\left(\varepsilon e^{-x_{\ell}+y_{\ell}}+1\right)^{-2 \imath \lambda},
\end{aligned}
$$

где

$$
Q_{C_{\ell-1}^{(1)}}^{D_{\ell}^{(1)}}\left(\underline{x}^{(\ell)}, \underline{z}^{(\ell-1)}\right)=\exp \left\{-e^{z_{1}+x_{1}}-\sum_{i=1}^{\ell-1}\left(e^{z_{i}-x_{i}}+e^{x_{i+1}-z_{i}}\right)-g e^{-x_{\ell}-z_{\ell-1}}\right\}
$$

И

$$
\begin{gathered}
Q_{D_{\ell}^{(1)}}^{C_{\ell-1}^{(1)}}\left(z_{1}, \ldots, z_{\ell-1}, y_{1}, \ldots, y_{\ell} ; \varepsilon\right)=\exp \left\{-e^{z_{1}+y_{1}}-\sum_{i=1}^{\ell-2}\left(e^{z_{i}-y_{i}}+e^{y_{i+1}-z_{i}}\right)\right. \\
\left.-e^{z_{\ell-1}-y_{\ell-1}}-\varepsilon e^{y_{\ell}-z_{\ell-1}}-\varepsilon^{-1} g e^{-x_{\ell}-z_{\ell-1}}\right\}
\end{gathered}
$$

Эти операторы получаются сдвигом переменной $y_{\ell}=y_{\ell}+\ln \varepsilon$ в (1.247)-(1.249). Тогда имеет место следующее соотношение между $\mathscr{Q}$-оператором для $D_{\ell}^{(1)}$-цепочки Тоды и (модифицированным) оператором рекурсии для $\mathfrak{s o}_{2 \ell}$-функции Уиттекера:

$$
Q_{D_{\ell-1} \oplus D_{1}}^{D_{\ell}}\left(\underline{x}^{(\ell)}, \underline{y}^{(\ell)} ; \lambda\right)=\lim _{\substack{\varepsilon \rightarrow 0, \varepsilon^{-1} g \rightarrow 0}} \varepsilon^{-\imath \lambda} Q^{D_{\ell}^{(1)}}\left(\underline{x}^{(\ell)}, \underline{y}^{(\ell)} ; \lambda ; \varepsilon\right) .
$$

\section{2. Часть 2. Доказательства}

Пусть $G$ - комплексная связная односвязная полупростая группа Ли ранга $\ell$ и $\mathfrak{g}=\operatorname{Lie}(G)$ - отвечающая ей полупростая алгебра Ли с образующими Шевалле $f_{i}, h_{i}, e_{i}$. Зафиксируем борелевскую подгруппу $B_{+}$и максимальный тор $T \subset B_{+}$. Эти данные определяют пару $N_{+}, N_{-}$противоположных унипотентных подгрупп группы $G, N_{+} \subset B_{+}$. Пусть $\Gamma$ - множество вершин графа Дынкина алгебры Ли $\mathfrak{g},\left\{\alpha_{i}, i \in \Gamma\right\}-$ множество простых корней, $\left\{\gamma_{k}, k=1, \ldots, m\right\}$, $m=\frac{1}{2} \operatorname{dim} \mathfrak{g} / \mathfrak{h},-$ положительные корни и $\left\{\alpha_{i}^{\vee}, i \in \Gamma\right\}-$ множество простых кокорней. Для каждого $i \in \Gamma$ имеется групповой гомоморфизм

$$
\varphi_{i}: \operatorname{SL}(2) \longrightarrow G
$$


который определен следующим образом. Рассмотрим множество однопараметрических подгрупп $e^{t f_{i}}=X_{i}(t) \subset N_{+}, e^{t e_{i}}=Y_{i}(t) \subset N_{-}$и $e^{t h_{i}}=\alpha_{i}^{\vee}(t) \subset T$ и положим

$$
\varphi_{i}\left(e^{t e}\right)=e^{t e_{i}}, \quad \varphi_{i}\left(e^{t f}\right)=e^{t f_{i}}, \quad \varphi_{i}\left(e^{t h}\right)=\alpha_{i}^{\vee}(t),
$$

где $e, f, h$ - стандартные образующие алгебры Ли $\mathfrak{s l}_{2}$. Зафиксируем подъемы $\dot{s}_{i} \subset G, \dot{s} \subset \mathrm{SL}(2)$ образующих $s_{i}$ группы Вейля группы $G$ и образующего группы Вейля группы $\mathrm{SL}(2)$ :

$$
\dot{s}=e^{f} e^{-e} e^{f}, \quad \dot{s}_{i}=e^{f_{i}} e^{-e_{i}} e^{f_{i}} .
$$

Так определенные подъемы групповых образующих очевидным образом согласованы с гомоморфизмами $(2.1)$, т. е. $\varphi_{i}(\dot{s})=\dot{s}_{i}$. Имеют место следующие соотношения:

$$
\dot{s}^{-1} e \dot{s}=-f, \quad \dot{s}_{i}^{-1} e_{i} \dot{s}_{i}=-f_{i}
$$

Действие $w_{0}\left(\alpha_{i}\right)=-\alpha_{i *}$ элемента максимальной длины $w_{0}$ группы Вейля на простых корнях определяет инволюцию $i \mapsto i^{*}$. Соответствующее действие элемента $\dot{w}_{0}$ задается как

$$
\dot{w}_{0}^{-1} e_{i} \dot{w}_{0}=-f_{i^{*}}
$$

ЗАмечАниЕ 2.1. Для классических групп Ли имеем: $i^{*}=\ell+1-i$ для $G=\mathrm{SL}(\ell+1), i^{*}=i$ для $G=\mathrm{SO}(2 \ell+1)$ и для $G=\operatorname{Sp}(2 \ell)$. В случае $G=\mathrm{SO}(2 \ell)$ $($ при $\ell \geqslant 2)$ действие инволюции $*$ имеет вид:

$$
\begin{gathered}
*: \quad 1 \longmapsto\left\{\begin{array} { l } 
{ 1 , \quad \ell \text { четно, } } \\
{ 2 , \quad \ell \text { нечетно, } }
\end{array} \quad 2 \longmapsto \left\{\begin{array}{ll}
2, & \ell \text { четно, } \\
1, & \ell \text { нечетно, }
\end{array}\right.\right. \\
k^{*}=k, \quad 2<k \leqslant \ell,
\end{gathered}
$$

где нумерация корней группы $\mathrm{SO}(2 \ell)$ совпадает с (1.192).

Ниже мы рассматриваем матричные элементы конечномерных представлений $V_{\omega_{i}}$ алгебры Ли $\mathfrak{g}$, отвечающих фундаментальным весам $\omega_{i}, i \in \Gamma$. Пусть $\xi_{\omega_{i}}^{+}$ и $\xi_{\omega_{i}}^{-}-$старший и младший векторы в $V_{\omega_{i}}$, определенные условием $\mathfrak{n}_{ \pm} \xi_{i}^{ \pm}=0$ и нормализованные так, что $\left\langle\xi_{\omega_{i}}^{-} \mid \xi_{\omega_{i}}^{+}\right\rangle=1$. Для подъема (2.3) элементов группы Вейля мы имеем (см., например, [24; лемма 3.8], [15; формула (2.29)])

$$
\dot{w}_{0}^{-1} \xi_{\omega_{i}}^{+}=\xi_{\omega_{i}}^{-}, \quad \dot{s}_{i}^{-1} \xi_{\omega_{i}}^{+}=e_{i} \xi_{\omega_{i}}^{+}
$$

Рассмотрим следующую параметризацию типичного элемента $g \in G$ :

$$
g=g^{(-)} g^{(0)} g^{(+)}=\exp \left(\sum_{\alpha \in \Phi_{+}} u_{-\alpha} e_{\alpha}\right) \exp \left(\sum_{i=1}^{\ell} u_{i} h_{i}\right) \exp \left(\sum_{\alpha \in \Phi_{+}} u_{\alpha} f_{\alpha}\right)
$$

Для координат $u_{i}$, соответствующих образующим Картана $h_{i}$, и для координат $u_{ \pm \alpha_{i}}$, соответствующих образующим $e_{\alpha_{i}}, f_{\alpha_{i}}$, которые связаны с простыми 
корнями, имеются простые выражения в терминах матричных элементов фундаментальных представлений $V_{\omega_{i}}$ :

$$
\begin{gathered}
u_{\alpha_{i}}(g)=\frac{\left\langle\xi_{\omega_{i}}^{-}\left|\pi_{i}(g) \pi_{i}\left(e_{i}\right)\right| \xi_{\omega_{i}}^{+}\right\rangle}{\left\langle\xi_{\omega_{i}}\left|\pi_{i}(g)\right| \xi_{\omega_{i}}^{+}\right\rangle}, \quad u_{-\alpha_{i}}(g)=\frac{\left\langle\xi_{\omega_{i}}^{-}\left|\pi_{i}\left(f_{i}\right) \pi_{i}(g)\right| \xi_{\omega_{i}}^{+}\right\rangle}{\left\langle\xi_{\omega_{i}}^{-}\left|\pi_{i}(g)\right| \xi_{\omega_{i}}^{+}\right\rangle}, \\
u_{i}(g)=\left\langle\xi_{\omega_{i}}^{-}\left|\pi_{i}(g)\right| \xi_{\omega_{i}}^{+}\right\rangle
\end{gathered}
$$

где $\pi_{i} \equiv \pi_{\omega_{i}}$ - фундаментальное представление в пространстве $V_{\omega_{i}}$. Определим обобщенные подкрученные миноры как

$$
\Delta_{\omega_{i}, \dot{w}}(g)=\left\langle\xi_{\omega_{i}}^{-}\left|\pi_{\omega_{i}}(g) \pi_{\omega_{i}}(\dot{w})\right| \xi_{\omega_{i}}^{+}\right\rangle, \quad g \in G .
$$

Тогда координаты $u_{i}$ и $u_{\alpha_{i}}$ подкрученного унипотентного элемента $v \dot{w}_{0}^{-1} \in G$ (где $v \in N_{+}$) можно выразить через подкрученные миноры (2.10) следующим образом:

$$
\begin{gathered}
e^{u_{i}\left(v \dot{w}_{0}^{-1}\right)}=\Delta_{\omega_{i}, \dot{w}_{0}^{-1}}(v) \\
u_{\alpha_{i}}\left(v \dot{w}_{0}^{-1}\right)=\frac{\left\langle\xi_{i}^{-}\left|\pi_{i}\left(v \dot{w}_{0}^{-1}\right) \pi_{i}\left(e_{i}\right)\right| \xi_{i}^{+}\right\rangle}{\left\langle\xi_{i}^{-}\left|\pi_{i}\left(v \dot{w}_{0}^{-1}\right)\right| \xi_{i}^{+}\right\rangle}=\frac{\left\langle\xi_{i}^{-}\left|\pi_{i}(v) \pi_{i}\left(\dot{w}_{0}^{-1}\right) \pi_{i}\left(\dot{s}_{i}^{-1}\right)\right| \xi_{\omega_{i}}^{+}\right\rangle}{\left\langle\xi_{i}^{-}\left|\pi_{i}\left(v \dot{w}_{0}^{-1}\right)\right| \xi_{\omega_{i}}^{+}\right\rangle} \\
=-\frac{\left\langle\xi_{\omega_{i}}^{-}\left|\pi_{i}(v) \pi_{i}\left(f_{i^{*}}\right) \pi_{i}\left(\dot{w}_{0}^{-1}\right)\right| \xi_{\omega_{i}}^{+}\right\rangle}{\left\langle\xi_{i}^{-}\left|\pi_{i}\left(v \dot{w}_{0}^{-1}\right)\right| \xi_{i}^{+}\right\rangle}=\frac{\Delta_{\omega_{i}, \dot{w}_{0}^{-1} \dot{s}_{i}^{-1}}(v)}{\Delta_{\omega_{i}, \dot{w}_{0}^{-1}}(v)}
\end{gathered}
$$

В дальнейшем мы используем следующие краткие обозначения:

$$
\begin{aligned}
& \Delta_{i}^{\prime}(v):=\left\langle\xi_{\omega_{i}}^{-}\left|\pi_{i}\left(v f_{i^{*}} \dot{w}_{0}^{-1}\right)\right| \xi_{\omega_{i}}^{+}\right\rangle=-\Delta_{\omega_{i}, \dot{w}_{0}^{-1} \dot{s}_{i}^{-1}}(v), \\
& \Delta_{i}(v):=\Delta_{\omega_{i}, \dot{w}_{0}^{-1}}(v) .
\end{aligned}
$$

2.1. Мера на $N_{+}$: доказательство леммы 1.2. В этом разделе мы получим явное выражение (1.21) для меры $d \mu_{N_{+}}(x)$ на унипотентной подгруппе $N_{+} \subset G$ в классической группе, используя факторизационную параметризацию (1.20) на $N_{+}$. Случай общей полупростой группы Ли следует из результатов работы [33].

Напомним, что для приведенного слова $I_{\ell}=\left(i_{1}, \ldots, i_{m_{\ell}}\right)$ элемента $w_{0}$ имеется бирациональный изоморфизм $\mathbb{C}^{m_{\ell}} \rightarrow N_{+}$. В частности, фиксируя унипотентный элемент $v \in N_{+}^{(0)}$, мы получаем факторизационное представление

$$
v(t)=X_{i_{1}}\left(t_{1}\right) X_{i_{2}}\left(t_{2}\right) \cdots X_{i_{m_{\ell}}}\left(t_{m_{\ell}}\right),
$$

где $X_{i}(t)=e^{t f_{i}}$. Переменные $t_{i}$ называются параметрами факторизации для элемента $v$.

ПредЛОЖенИЕ 2.1. Пустъ $v(t) \in N_{+}^{(0)}$ - факторизационная параметризаиия (2.13), отвечающая приведенному слову $I=\left(i_{1}, \ldots, i_{m_{\ell}}\right)$. Тогда мера

$$
d \mu_{N_{+}}(v(t))=\prod_{k=1}^{\ell} \prod_{i=1}^{m_{\ell}} t_{i}^{\left\langle\omega_{k}, \gamma_{i}\right\rangle} \cdot \bigwedge_{i=1}^{m_{\ell}} \frac{d t_{i}}{t_{i}}
$$

является ограничением правоинвариантной меры $d \mu_{N_{+}}$на $N_{+}^{(0)}$, m.е.

$$
d \mu_{N_{+}}(v(t))=d \mu_{N_{+}}\left(v(t) X_{j}(\tau)\right), \quad j=1, \ldots, \ell .
$$


ДокАЗАТЕЛЬСтво. Исследуем зависимость от выбора приведенного слова $I=\left(i_{1}, \ldots, i_{m_{\ell}}\right)$. Пусть $t^{I}=\left(t_{1}^{I}, \ldots, t_{m_{\ell}}^{I}\right)$ - параметры факторизации, отвечающие редуцированному слову $I$. Согласно [16; теорема 4.3], имеются следующие выражения для матричных элементов:

$$
\Delta_{k}\left(t^{I}\right):=\Delta_{k}\left(x\left(t^{I}\right) w_{0}^{-1}\right)=\prod_{i=1}^{m_{\ell}}\left(t_{i}^{I}\right)^{\left\langle\omega_{k}, \gamma_{i}\right\rangle} .
$$

Две параметризации $x\left(t^{I}\right)$ и $x\left(t^{I^{\prime}}\right)$ открытого множества $N_{+}^{(0)}$, отвечающие приведенным словам $I$ и $I^{\prime}$, связаны бирациональным преобразованием.

ЛЕмма 2.1. Для двух параметризаиий $x\left(t^{I}\right)$ u $x\left(t^{I^{\prime}}\right)$, соответствующих приведенным словам I и $I^{\prime}$, имеют место следующие утверждения.

1. Соответствующие матричные элементы совпадают:

$$
\Delta_{k}\left(t^{I}\right)=\Delta_{k}\left(t^{I^{\prime}}\right), \quad 1 \leqslant k \leqslant \ell .
$$

2. Следующие дифференииаль совпадают:

$$
\bigwedge_{j=1}^{m_{\ell}} \frac{d t_{j}^{I}}{t_{j}^{I}}=\bigwedge_{j=1}^{m_{\ell}} \frac{d t_{j}^{I^{\prime}}}{t_{j}^{I^{\prime}}}
$$

ДоКАЗАТЕЛЬСтво ЛЕммы. Как показано в [14], бирациональные преобразования $R_{I}^{I^{\prime}}$ на $N_{+}$, отвечающие любой паре приведенных слов $I$ и $I^{\prime}$, можно представить в виде композиции элементарных преобразований (так называемых 3-, 4- и 6-движений). Поэтому для доказательства (2.17), (2.18) надо проверить тождества для элементарных движений только в следующих случаях. А именно, в случае классических групп Ли достаточно рассматривать следующие два бирациональные преобразования $R_{I}^{I^{\prime}}: t^{I} \rightarrow t^{I^{\prime}}$ :

1) $X_{i}\left(t_{1}\right) X_{j}\left(t_{2}\right) X_{i}\left(t_{3}\right)=X_{j}\left(t_{1}^{\prime}\right) X_{i}\left(t_{2}^{\prime}\right) X_{j}\left(t_{3}^{\prime}\right)$ при $a_{i j}=a_{j i}=-1$,

2) $X_{j}\left(t_{1}\right) X_{i}\left(t_{2}\right) X_{j}\left(t_{3}\right) X_{i}\left(t_{4}\right)=X_{i}\left(t_{1}^{\prime}\right) X_{j}\left(t_{2}^{\prime}\right) X_{i}\left(t_{3}^{\prime}\right) X_{j}\left(t_{4}^{\prime}\right)$ при $a_{i j}=-1$ и $a_{j i}=$ -2 , где мы обозначаем $t=t^{I}$ и $t^{\prime}=t^{I^{\prime}}$.

Доказательство тождества (2.17) для элементарных 3-, 4- и 6-движений следует непосредственно из результатов работы [16]. Для полноты изложения достаточно проверить (2.18) для 3- и 4-движений.

1) В случае $a_{i j}=a_{j i}=-1$ мы должны рассмотреть бирациональное преобразование между параметризациями, отвечающими приведенным словам $I=$ $(\ldots i j i \ldots)$ и $I^{\prime}=(\ldots j i j \ldots)$. Имеется следующее соотношение между параметрами:

$$
v=X_{i}\left(t_{1}\right) X_{j}\left(t_{2}\right) X_{i}\left(t_{3}\right)=X_{j}\left(t_{1}^{\prime}\right) X_{i}\left(t_{2}^{\prime}\right) X_{j}\left(t_{3}^{\prime}\right),
$$

где

$$
t_{1}^{\prime}=\frac{t_{2} t_{3}}{t_{1}+t_{3}}, \quad t_{2}^{\prime}=t_{1}+t_{3}, \quad t_{3}^{\prime}=\frac{t_{1} t_{2}}{t_{1}+t_{3}} .
$$

Непосредственная проверка показывает, что

$$
d \ln t_{1}^{\prime} \wedge d \ln t_{2}^{\prime} \wedge d \ln t_{3}^{\prime}=d \ln t_{1} \wedge d \ln t_{2} \wedge d \ln t_{3}
$$


2) В случае $a_{i j}=-1, a_{j i}=-2$ мы должны рассмотреть бирациональное преобразование между параметризациями, отвечающими приведенным словам $I=(\ldots j i j i \ldots)$ и $I^{\prime}=(\ldots i j i j \ldots)$. Тем самым, мы получаем следующее соотношение между параметрами:

$$
X=X_{j}\left(t_{1}\right) X_{i}\left(t_{2}\right) X_{j}\left(t_{3}\right) X_{i}\left(t_{4}\right)=X_{i}\left(t_{1}^{\prime}\right) X_{j}\left(t_{2}^{\prime}\right) X_{i}\left(t_{3}^{\prime}\right) X_{j}\left(t_{4}^{\prime}\right),
$$

где

$$
\begin{aligned}
t_{1}^{\prime} & =\frac{t_{2} t_{3}^{2} t_{4}}{t_{1}^{2} t_{2}+\left(t_{1}+t_{3}\right)^{2} t_{4}}, & t_{2}^{\prime} & =\frac{t_{1}^{2} t_{2}+\left(t_{1}+t_{3}\right)^{2} t_{4}}{t_{1} t_{2}+\left(t_{1}+t_{3}\right) t_{4}}, \\
t_{3}^{\prime} & =\frac{\left(t_{1} t_{2}+\left(t_{1}+t_{3}\right) t_{4}\right)^{2}}{t_{1}^{2} t_{2}+\left(t_{1}+t_{3}\right)^{2} t_{4}}, & t_{4}^{\prime} & =\frac{t_{1} t_{2} t_{3}}{t_{1} t_{2}+\left(t_{1}+t_{3}\right) t_{4}} .
\end{aligned}
$$

Можно непосредственно проверить следующее тождество:

$$
d \ln t_{1}^{\prime} \wedge d \ln t_{2}^{\prime} \wedge d \ln t_{3}^{\prime} \wedge d \ln t_{4}^{\prime}=d \ln t_{1} \wedge d \ln t_{2} \wedge d \ln t_{3} \wedge d \ln t_{4}
$$

Это завершает доказательство леммы.

Сейчас мы готовы завершить доказательство предложения 2.1. Чтобы установить правоинвариантность меры $d \mu_{N_{+}}(v)$, мы используем $(2.17),(2.18)$. Для любого простого корня $\alpha_{i}$ можно найти приведенное слово $I\left(\alpha_{i}\right)=\left(j_{1}, \ldots, j_{m}\right)$ элемента $w_{0}$ с $m=m_{\ell}$, для которого $j_{m}=i$. Тогда из тождеств $(2.17),(2.18)$ следует, что

$$
d \mu_{N_{+}}\left(v\left(t^{I\left(\alpha_{i}\right)}\right)\right)=d \mu_{N_{+}}\left(v\left(t^{I_{\ell}}\right)\right) .
$$

Таким образом, мы получаем

$$
v\left(t^{I\left(\alpha_{i}\right)}\right) \cdot X_{i}(\tau)=X_{j_{1}}\left(t_{1}\right) X_{j_{m-1}}\left(t_{m-1}\right) X_{i}\left(t_{m}+\tau\right) .
$$

По построению параметр факторизации $t_{m}$ входит только в (мономиальное) выражение для $\Delta_{j}(v(t))$ как однородный множитель первой степени. Тем самым, параметр факторизации $t_{m}$ возникает в выражении для меры $d \mu_{N_{+}}$только в $\alpha_{j}$-компоненте $\Delta_{j}(v(t)) d \ln t_{m}$, а значит, мера $d \mu_{N_{+}}$инвариантна относительно сдвига $t_{m} \rightarrow t_{m}+\tau$. Итак, мера правоинвариантна относительно действия $X_{j}(\tau)$ для любого $j=1, \ldots, \ell$ и, значит, правоинвариантна относительно всей группы $N_{+}$. Это завершает доказательство предложения 2.1, а с учетом (2.16) и доказательство леммы 1.2 .

\section{2. Векторы Уиттекера для классических групп Ли: доказатель-} ства леммы 1.1 и предложения 1.1. В этом разделе мы получим выражения для левого и правого g-векторов Уиттекера в терминах матричных элементов конечномерных представлений алгебры g. Векторы Уиттекера удовлетворяют следующим уравнениям:

$$
f_{i} \psi_{R}=-\psi_{R}, \quad e_{i} \psi_{L}=-\psi_{L}, \quad i=1, \ldots, \ell .
$$

Интегрируя действия нильпотентных подалгебр Ли $\mathfrak{n}_{ \pm} \subset \mathfrak{g}$ до действий нильпотентных подгрупп Ли $N_{ \pm} \subset G$, уравнения на g-функции Уиттекера можно переписать в терминах однопараметрических подгрупп $X_{i}(t) \subset N_{+}$и $Y_{i}(t) \subset N_{-}$ следующим образом:

$$
\pi_{\mu}\left(X_{i}(t)\right) \psi_{R}(v)=e^{-t} \psi_{R}(v), \quad \pi_{\mu}\left(Y_{i}(t)\right) \psi_{L}(v)=e^{-t} \psi_{L}(v),
$$


где $i=1, \ldots, \ell, v \in N_{+}$. Эквивалентно, для любого $z_{ \pm} \in N_{ \pm}$имеем

$$
\begin{aligned}
& \pi_{\mu}\left(z_{+}\right) \psi_{R}(v)=\exp \left\{-\sum_{i=1}^{\ell}\left(z_{+}\right)_{i}\right\} \psi_{R}(v), \\
& \pi_{\lambda}\left(z_{-}\right) \psi_{L}(v)=\exp \left\{-\sum_{i=1}^{\ell}\left(z_{-}\right)_{i}\right\} \psi_{L}(v),
\end{aligned}
$$

где $\left(z_{ \pm}\right)_{i}:=u_{ \pm \alpha_{i}}\left(z_{ \pm}\right)$. Конструкция правого вектора Уиттекера получается непосредственно. Заметим, что имеют место простые тождества

$$
u_{\alpha_{i}}\left(v_{1} v_{2}\right)=u_{\alpha_{i}}\left(v_{1}\right)+u_{\alpha_{i}}\left(v_{2}\right), \quad v_{1}, v_{2} \in N_{+} .
$$

Тогда из (2.23) мы заключаем, что правый вектор Уиттекера задается мультипликативным характером максимальной унипотентной подгруппы $N_{+}$:

$$
\psi_{R}(v)=\exp \left\{-\sum_{i=1}^{\ell} v_{i}\right\}=\exp \left\{-\frac{\Delta_{\omega_{i}, \dot{s}_{i}^{-1}}(v)}{\Delta_{\omega_{i}, 1}(v)}\right\}, \quad v \in N_{+},
$$

где $v_{i}:=u_{\alpha_{i}}(v)$ и мы используем $(2.9)$ чтобы выразить $v_{i}$ через матричные элементы.

Для построения левого вектора Уиттекера в терминах матричных элементов применим внутренний автоморфизм группы $G$, действующий на $z \in G$ как $z^{\tau}=\dot{w}_{0}^{-1} z \dot{w}_{0}$. Принимая во внимание, что $\dot{w}_{0}^{-1} X_{i^{*}}(-t) \dot{w}_{0}=Y_{i}(t)$, мы получаем $\dot{w}_{0}^{-1} N_{+} \dot{w}_{0}=N_{-}$. Теперь уравнение на левый вектор Уиттекера

$$
\pi_{\mu}\left(Y_{i}(t)\right) \psi_{L}(v)=e^{-t} \psi_{L}(v), \quad i=1, \ldots, \ell,
$$

может быть записано в виде

$$
\pi_{\mu}\left(z^{\tau}\right) \psi_{L}(v)=\exp \left\{-\sum_{i=1}^{\ell} z_{i}\right\} \psi_{L}(v), \quad z \in N_{+} .
$$

Левый вектор Уиттекера можно выразить через правый вектор:

$$
\psi_{L}(v)=\psi_{R}\left(v \dot{w}_{0}^{-1}\right)^{-1},
$$

где функция $\psi_{R}$ рассматривается как $B_{-}$-эквивариантная функция на $G$ (точное определение дано в (1.9)). Используя разложение Гаусса и параметризацию (2.8), (2.9), мы получаем для левого вектора Уиттекера

$$
\psi_{L}(v)=\exp \left\langle\imath \lambda-\rho, \sum_{i=1}^{\ell} u_{i}\left(v \dot{w}_{0}^{-1}\right) h_{i}\right\rangle \exp \left\{\sum_{i=1}^{\ell} u_{\alpha_{i}}\left(v \dot{w}_{0}^{-1}\right)\right\} .
$$

В терминах матричных элементов конечномерных представлений имеет место представление

$$
\begin{aligned}
\psi_{L}(v) & =\prod_{i=1}^{\ell} \Delta_{\omega_{i}, \dot{w}_{0}^{-1}}(v)^{\left\langle\imath \lambda-\rho, \alpha_{i}^{\vee}\right\rangle} \exp \left\{\frac{\Delta_{\omega_{i}, \dot{w}_{0}^{-1} \dot{s}_{i}^{-1}(v)}}{\Delta_{\omega_{i}, \dot{w}_{0}^{-1}}(v)}\right\} \\
& =\prod_{i=1}^{\ell} \Delta_{i}(v)^{\left\langle\imath \lambda-\rho, \alpha_{i}^{\vee}\right\rangle} \exp \left\{-\frac{\Delta_{i}^{\prime}(v)}{\Delta_{i}(v)}\right\}
\end{aligned}
$$


Это завершает доказательство леммы 1.1. Доказательство предложения 1.1 можно получить, комбинируя выражения для правого вектора Уиттекера и левого вектора Уиттекера, подкрученных соответственно на элементы $e^{H_{L}}$ и $e^{H_{R}}$ максимального тора, такие, что $e^{H_{L}+H_{R}}=\exp \left(-h_{x}\right)=\exp \left\{-\sum_{i=1}^{\ell}\left\langle\omega_{i}, x\right\rangle h_{i}\right\}$.

2.3. Явные вычисления для матричных элементов. Для построения интегральных представлений функций Уиттекера надо выразить различные матричные элементы, входящие в интегральную формулу (1.19), используя факторизационную и модифицированную факторизационную параметризации элементов группы. Это можно сделать непосредственно, применяя результаты работ [16], [15]. Ниже мы используем рекурсивную структуру приведенного слова $I_{\ell}$, отвечающего элементу максимальной длины $w_{0}$ в группе Вейля $W\left(X_{\ell}\right)$ классической алгебры Ли типа $X_{\ell}$ для $X=A, B, C, D$. Эта рекурсивная структура приводит к рекурсивным формулам для подходящих отношений матричных элементов. Решая рекурсивные уравнения, мы находим явные выражения для $\psi_{L}$ и $\psi_{R}$ в (модифицированной) факторизационной параметризации. Это дает соответствующие интегральные представления для функций Уиттекера классических групп Ли. В случае модифицированной факторизационной параметризации мы получаем обобщение интегрального представления Гивенталя для $\mathfrak{g}=\mathfrak{g l}_{\ell+1}$ на остальные алгебры Ли классического типа.

2.3.1. Выражения для $\mathfrak{g l}_{\ell+1}$-матричных элементов: доказательства теоремы 1.1 и теоремы 1.3. В этом пункте мы находим выражения для матричных элементов, которые связаны с построением интегральных представлений для $\mathfrak{g l}_{\ell+1}$-функций Уиттекера, используя факторизационную параметризацию открытого подмножества $N_{+} \subset \mathrm{GL}(\ell+1)$. Это дает доказательство интегральных представлений $\mathfrak{g l}_{\ell+1}$-функций Уиттекера, анонсированных в части 1.

Собственные функции $\mathfrak{g l}_{\ell+1}-$ и $\mathfrak{s l}_{\ell+1}$-цепочек Тоды отличаются простым множителем (1.31), и векторы Уиттекера $\psi_{L}, \psi_{R}$ совпадают для обеих алгебр Ли. Мы используем корневые данные для $\mathfrak{s l}_{\ell+1}$ для вычисления матричных элементов $\Delta_{\omega_{i}, \dot{w}_{0}^{-1}}(v), \Delta_{\omega_{i}, \dot{w}_{0}^{-1} \dot{s}_{i}^{-1}}(v), i=1, \ldots, \ell$, в фундаментальных представлениях алгебры Ли $\mathfrak{s l}_{\ell+1}$ и дополнительно полагаем $\Delta_{\omega_{\ell+1}, \dot{w}_{0}^{-1}}(v)=1$. Корневые данные для $\mathfrak{s l}_{\ell+1}$ указаны в (1.28). Разложение $w_{0}=s_{i_{1}} s_{i_{2}} \cdots s_{i_{m}}$ элемента максимальной длины $w_{0} \in S_{\ell+1}$, соответствующее приведенному слову $I_{\ell}=\left(i_{1}, \ldots, i_{m}\right)$ при $m=m_{\ell}=\ell(\ell+1) / 2$, определяет упорядочение на положительных кокорнях: $R_{+}^{\vee}=\left\{\gamma_{k}^{\vee}=s_{i_{1}} \cdots s_{i_{k-1}} \alpha_{k}^{\vee}, k=1, \ldots, m_{\ell}\right\}$. Мы рассматриваем разложение элемента $w_{0}$, соответствующее следующему приведенному слову:

$$
I_{\ell}=(1,21, \ldots,(\ell \ldots 21))
$$

Соответствующее упорядочение положительных кокорней имеет вид:

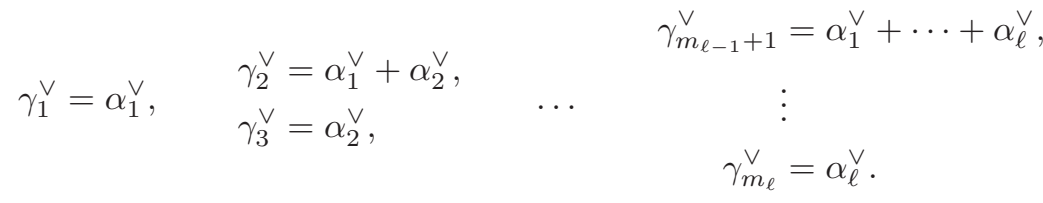


Рекурсивная параметризация открытого подмножества $N_{+}^{(0)}$ в $N_{+}$, соответствующая приведенному слову $I_{\ell}$, приобретает следующий вид. Для $v^{A_{\ell}} \in N_{+}^{(0)}$ мы имеем

$$
v^{A_{\ell}}(y)=\mathfrak{X}_{1}(y) \mathfrak{X}_{2}(y) \cdots \mathfrak{X}_{\ell}(y),
$$

где

$$
\mathfrak{X}_{k}(y)=X_{k}\left(y_{k, n_{k, k}}\right) \cdots X_{2}\left(y_{2, n_{k, 2}}\right) X_{1}\left(y_{1, n_{k, 1}}\right)
$$

и $\mathfrak{X}_{1}=X_{1}\left(y_{1,1}\right)$. Здесь использованы следующие обозначения. Пусть $\left|I_{\ell}\right|=$ $m_{\ell}$ - длина элемента $w_{0}$. Для системы корней типа $A_{\ell}$ имеем $m_{\ell}=\ell(\ell+1) / 2$. Тогда для любого $k \in\{1, \ldots, \ell\}$ рассмотрим подслово

$$
I_{k}=\left(i_{1}, \ldots, i_{m_{k}}\right) \subset I_{\ell}=\left(i_{1}, \ldots, i_{m_{k}}, i_{m_{k}+1}, \ldots, i_{m_{\ell}}\right),
$$

где $\left|I_{k}\right|=m_{k}=k(k+1) / 2$. Пусть $A_{k}$ - соответствующая корневая подсистема в $R_{+}$и $v^{A_{k}}=\mathfrak{X}_{1} \cdots \mathfrak{X}_{k}-$ факторизационная параметризация на соответствующей подгруппе. Параметры факторизации для $v^{A_{k}}(y)$ естественно пронумеровать так: $\left\{y_{i, n} ; 1 \leqslant i \leqslant k, 1 \leqslant n \leqslant n_{k, i}\right\}$ и

$$
n_{k, i}=k+1-i, \quad 1 \leqslant i \leqslant \ell .
$$

Мы интересуемся явными выражениями для следующих матричных элементов в терминах параметров факторизации $\left\{y_{i, n}\right\}$ :

$$
\Delta_{i}(v):=\left\langle\xi_{\omega_{i}}^{-}\left|\pi_{i}\left(v \dot{w}_{0}^{-1}\right)\right| \xi_{\omega_{i}}^{+}\right\rangle, \quad \Delta_{i}^{\prime}(v):=\left\langle\xi_{\omega_{i}}^{-}\left|\pi_{i}\left(v f_{i^{*}} \dot{w}_{0}^{-1}\right)\right| \xi_{\omega_{i}}^{+}\right\rangle,
$$

где $\pi_{i}=\pi_{\omega_{i}}, i=1, \ldots, \ell,-$ фундаментальное представление со старшим весом $\omega_{i}$, а $\xi_{\omega_{i}}^{+}$и $\xi_{\omega_{i}}^{-}-$старший и младший весовые векторы в представлениях $\pi_{i}$, определенные условиями $\mathfrak{n}_{ \pm} \xi_{i}^{ \pm}=0$ и нормализованные так, что $\left\langle\xi_{\omega_{i}}^{+} \mid \xi_{\omega_{i}}^{-}\right\rangle=1$. Заметим, что в соответствии с (2.5) для алгебры Ли $\mathfrak{s l}_{\ell+1}$ мы получаем $i^{*}=\ell+1-i$. Доказательство следующего утверждения получается последовательным вычислением матричных элементов с учетом соотношений Серра и определяющих идеалов для фундаментальных представлений с использованием техники работы [16].

Лемма 2.2. При $i=1, \ldots, \ell$ имеют место следующие рекурсивные соотношения:

$$
\begin{aligned}
\Delta_{i}(v)^{A_{\ell}} & =\left(\prod_{k=1}^{i} y_{\ell+1-k, i}\right) \Delta_{i}(v)^{A_{\ell-1}} \\
\left(\frac{\Delta_{i}^{\prime}(v)}{\Delta_{i}(v)}\right)^{A_{\ell}} & =\frac{1}{y_{\ell+1-i, i}}+\left(1-\delta_{i, \ell}\right) \frac{y_{\ell-i, i+1}}{y_{\ell+1-i, i}}\left(\frac{\Delta_{i}^{\prime}(v)}{\Delta_{i}(v)}\right)^{A_{\ell-1}} .
\end{aligned}
$$

Матричные элементы теперь можно найти, решая рекурсивные соотношения (2.30).

Лемма 2.3. Пусть v определено формулами (1.32), (1.33). Тогда при $i=$ $1, \ldots, \ell$ имеют место следуюшие соотношения на матричные элементы $v$ в терминах переменных $y_{i, k}$ :

$$
\Delta_{\omega_{i}, \dot{s}_{i}}(v)^{A_{\ell}}=\sum_{n=i}^{\ell} y_{i, n},
$$




$$
\begin{gathered}
\Delta_{i}(v)^{A_{\ell}}=\prod_{k=i}^{\ell} \prod_{n=1}^{i} y_{k+1-n, n}, \\
\left(\frac{\Delta_{k}^{\prime}(v)}{\Delta_{i}(v)}\right)^{A_{\ell}}=\frac{1}{y_{\ell+1-i, i}}\left[1+\left(1-\delta_{i, \ell}\right) \sum_{n=1}^{\ell-i} \prod_{k=1}^{n} \frac{y_{\ell+1-k-i, i+1}}{y_{\ell+1-k-i, i}}\right] .
\end{gathered}
$$

Подставляя выражение (2.31) в (1.17), (1.18) и комбинирую результат с выражением (1.21) для меры, мы собираем полученные выражения в (1.19) и таким образом получаем интегральную формулу (1.37), что и завершает доказательство теоремы 1.1.

Рассмотрим интегральное представление для $\mathfrak{g l}_{\ell+1}$-функции Уиттекера в модифицированной факторизационной параметризации (1.41). Начнем с аналога рекурсивных соотношений (2.30) для матричных элементов в модифицированной факторизационной параметризации. Для упрощения вида рекурсивных соотношений оказывается полезным рассматривать модифицированную версию

$$
y_{i, n}=e^{x_{\ell+1, i}-x_{\ell+1, i+1}} e^{x_{n+i, i+1}-x_{n+i-1, i}}
$$

факторизационной параметризации (1.41) с учетом действия части $e^{H_{R}}(1.46)$ элемента максимального тора $e^{-h_{x}}$. Замена переменных $(2.32)$, примененная к (2.30), приводит к следующему.

ЛЕмма 2.4. 1) В модифицированной факторизащионной параметризащии (2.32) рекурсивные соотношения (2.30) имеют вид

$$
\left(\frac{\Delta_{i}^{\prime}(v)}{\Delta_{i}(v)}\right)^{A_{\ell}}=e^{x_{\ell, \ell-i+1}-x_{\ell+1, \ell-i+1}}+\frac{e^{x_{\ell, \ell-i+1}-x_{\ell, \ell-i}}}{e^{x_{\ell+1, \ell-i+1}-x_{\ell+1, \ell-i}}}\left(\frac{\Delta_{i}^{\prime}(v)}{\Delta_{i}(v)}\right)^{A_{\ell-1}} .
$$

2) Решение рекурсивных уравнений можно записать как

$$
\begin{aligned}
\left(\frac{\Delta_{i}^{\prime}(v)}{\Delta_{i}(v)}\right)^{A_{\ell}} & =\sum_{n=1}^{\ell+1-i} e^{x_{n+i-1, n}-x_{n+i, n}}, \\
\Delta_{\omega_{i}, \dot{w}_{0}^{-1}}(v)^{A_{\ell}} & =\exp \left\{\sum_{n=1}^{i}\left(x_{\ell+1, n}-x_{i, n}\right)\right\}, \\
\left(\frac{\Delta_{i}(v)}{\Delta_{i+1}(v)}\right)^{A_{\ell}} & =e^{-x_{\ell+1, i+1} \exp \left\{\sum_{k=1}^{i+1} x_{i+1, k}-\sum_{k=1}^{i} x_{i, k}\right\},}
\end{aligned}
$$

где $i=1, \ldots, \ell u \Delta_{\ell+1}(v)=1$.

Подставляя (2.34), (2.35) в (1.17), (1.18), мы получаем векторы Уиттекера в параметризации (2.32). Полагая $x_{\ell, k}=0, k=1, \ldots, \ell+1$, мы приходим к выражениям для векторов Уиттекера из леммы 1.4. Для того чтобы доказать теорему 1.3 , остается учесть вид меры $d \mu_{N_{+}}$в модифицированной факторизационной параметризации. Это завершает доказательство теоремы 1.3. 
2.3.2. Выражения для $\mathfrak{s o}_{2 \ell+1}$-матричных элементов: доказательства теоремы 1.4 и теоремы 1.6. В этом пункте мы приводим выражения для матричных элементов, возникающих в конструкции интегральных представлений $\mathfrak{s o}_{2 \ell+1}$ функций Уиттекера. Здесь используется факторизационная параметризация открытого подмножества в $N_{+} \subset \mathrm{SO}(2 \ell+1)$. Это дает доказательство интегральных представлений для $\mathfrak{s o}_{2 \ell+1}$-функций Уиттекера, анонсированных в части 1.

Мы используем корневые данные, описанные в (1.64). Разложение $w_{0}=$ $s_{i_{1}} s_{i_{2}} \cdots s_{i_{m_{\ell}}}$ элемента максимальной длины $w_{0} \in W\left(B_{\ell}\right)$, отвечающее приведенному слову $I_{\ell}=\left(i_{1}, \ldots, i_{m_{\ell}}\right)$ с $m_{\ell}=\ell^{2}$, определяет следующее упорядочение на множестве положительных корней: $R_{+}^{\vee}=\left\{\gamma_{k}^{\vee}=s_{i_{1}} \cdots s_{i_{k-1}} \alpha_{k}^{\vee}\right.$, $\left.k=1, \ldots, m_{\ell}\right\}$ для алгебры $\mathfrak{s o}_{2 \ell+1}$. Мы рассматриваем разложение элемента $w_{0}$, описываемое следующим приведенным словом:

$$
I_{\ell}=(1,212, \ldots,(\ell \ldots 212 \ldots \ell)) .
$$

Соответствующее упорядочение положительных кокорней задано как

$$
\begin{aligned}
& \gamma_{2}^{\vee}=\alpha_{1}^{\vee}+\alpha_{2}^{\vee} \\
& \gamma_{1}^{\vee}=\alpha_{1}^{\vee}, \quad \gamma_{3}^{\vee}=\alpha_{1}^{\vee}+2 \alpha_{2}^{\vee} \text {, } \\
& \gamma_{4}^{\vee}=\alpha_{2}^{\vee} \\
& \gamma_{(\ell-1)^{2}+1}^{\vee}=\alpha_{1}^{\vee}+2\left(\alpha_{2}^{\vee}+\cdots+\alpha_{\ell-1}^{\vee}\right)+\alpha_{\ell}^{\vee} \text {, } \\
& \gamma_{(\ell-1)^{2}+2}^{\vee}=\alpha_{1}^{\vee}+2\left(\alpha_{2}^{\vee}+\cdots+\alpha_{\ell-2}^{\vee}\right)+\alpha_{\ell-1}^{\vee}+\alpha_{\ell}^{\vee} \text {, }
\end{aligned}
$$

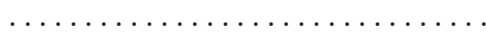

$$
\begin{aligned}
& \gamma_{\ell(\ell-1)}^{\vee}=\alpha_{1}^{\vee}+\alpha_{2}^{\vee}+\cdots+\alpha_{\ell}^{\vee}, \\
& \gamma_{\ell(\ell-1)+1}^{\vee}=\alpha_{1}^{\vee}+2\left(\alpha_{2}^{\vee}+\cdots+\alpha_{\ell}^{\vee}\right) \text {, } \\
& \gamma_{\ell(\ell-1)+2}^{\vee}=\alpha_{2}^{\vee}+\cdots+\alpha_{\ell}^{\vee} \text {, } \\
& \gamma_{\ell^{2}}^{\vee}=\alpha_{\ell}^{\vee}
\end{aligned}
$$

Рекурсивная параметризация открытого подмножества $N_{+}^{(0)}$ в $N_{+}$, соответствующая приведенному слову $I_{\ell}$, имеет следующий вид. Для $v^{B_{\ell}} \in N_{+}^{(0)}$ мы получаем

$$
v^{B_{\ell}}(y)=\mathfrak{X}_{1}(y) \mathfrak{X}_{2}(y) \cdots \mathfrak{X}_{\ell}(y),
$$

где

$$
\mathfrak{X}_{k}(y)=X_{k}\left(y_{k, n_{k, k}-1}\right) \cdots X_{2}\left(y_{2, n_{k, 2}-1}\right) X_{1}\left(y_{1, n_{k, 1}}\right) X_{2}\left(y_{2, n_{k, 2}}\right) \cdots X_{k}\left(y_{k, n_{k, k}}\right)
$$

и $\mathfrak{X}_{1}=X_{1}\left(y_{1,1}\right)$. Для любого $k \in\{1, \ldots, \ell\}$ рассмотрим подслово

$$
I_{k}=\left(i_{1}, \ldots, i_{m_{k}}\right) \subset I_{\ell}=\left(i_{1}, \ldots, i_{m_{k}}, i_{m_{k}+1}, \ldots, i_{m_{\ell}}\right)
$$


с $\left|I_{k}\right|=m_{k}=k^{2}$. Пусть $B_{k}$ - соответствующая подсистема корней в $R_{+}$и $v^{B_{k}}=\mathfrak{X}_{1} \cdots \mathfrak{X}_{k}-$ факторизационная параметризация на соответствующей подгруппе. Параметры факторизации для $v^{B_{k}}(y)$ можно естественно занумеровать элементами $\left\{y_{i, n} ; 1 \leqslant i \leqslant k, 1 \leqslant n \leqslant n_{k, i}\right\}$, где

$$
n_{k, 1}=k, \quad n_{k, i}=2(k+1-i), \quad 1<i \leqslant \ell .
$$

Также мы используем обозначение $n_{i}:=n_{\ell, i}$.

Мы интересуемся явными выражениями для матричных элементов в терминах параметров факторизации $\left\{y_{i, n}\right\}$ :

$$
\Delta_{i}(v):=\left\langle\xi_{\omega_{i}}^{-}\left|\pi_{i}\left(v \dot{w}_{0}^{-1}\right)\right| \xi_{\omega_{i}}^{+}\right\rangle, \quad \Delta_{i}^{\prime}(v):=\left\langle\xi_{\omega_{i}}^{-}\left|\pi_{i}\left(v f_{i^{*}} \dot{w}_{0}^{-1}\right)\right| \xi_{\omega_{i}}^{+}\right\rangle
$$

где $\pi_{i}=\pi_{\omega_{i}}$ - фундаментальное представление со старшим весом $\omega_{i}$, а $\xi_{\omega_{i}}^{+}$и $\xi_{\omega_{i}}^{-}-$ старший и младший векторы в представлении $\pi_{i}$, для которых $\left\langle\xi_{\omega_{i}}^{+} \mid \xi_{\omega_{i}}^{-}\right\rangle=1$. Заметим, что в случае алгебры Ли $\mathfrak{s o}_{2 \ell+1}$ мы имеем $i \rightarrow i^{*}$ для инволюции, определенной в (2.5). Доказательство следующего утверждения получается последовательным вычислением матричных элементов с учетом соотношений Серра и определяющих идеалов для фундаментальных представлений с использованием техники работы [16].

Лемма 2.5. Пусть $v:=v^{B_{\ell}}$, где $v^{B_{\ell}}$ определено в (2.37). Тогда имеют место следующие рекурсивные уравнения:

$$
\begin{aligned}
\Delta_{1}(v)^{B_{\ell}} & =\left(y_{1, \ell} \prod_{k=2}^{\ell} y_{k, n_{k}-1}\right) \cdot \Delta_{1}(v)^{B_{\ell-1}}, \\
\Delta_{i}(v)^{B_{\ell}} & =\left(y_{1, \ell}^{2} \prod_{k=2}^{i} y_{k, n_{k}-1} y_{k, n_{k}} \prod_{k=i+1}^{\ell} y_{k, n_{k}-1}^{2}\right) \cdot \Delta_{i}(v)^{B_{\ell-1},} \quad 1<i \leqslant \ell, \\
\left(\frac{\Delta_{1}^{\prime}(v)}{\Delta_{1}(v)}\right)^{B_{\ell}}= & \frac{1}{y_{1, \ell}}\left(1+\frac{y_{2,2(\ell-1)}}{y_{2,2(\ell-1)-1}}\right)+\frac{y_{2,2(\ell-1)}}{y_{2,2(\ell-1)-1}}\left(\frac{\Delta_{1}^{\prime}(v)}{\Delta_{1}(v)}\right)^{B_{\ell-1}}, \\
\left(\frac{\Delta_{k}^{\prime}(v)}{\Delta_{k}(v)}\right)^{B_{\ell}}= & \frac{1}{y_{k, 2}(\ell+1-k)}\left(1+\frac{y_{k+1,2(\ell-k)}}{y_{k+1,2(\ell-k)-1}}\right) \\
& +\frac{y_{k, 2(\ell+1-k)-1}}{y_{k, 2(\ell+1-k)}} \frac{y_{k+1,2(\ell-k)}}{y_{k+1,2(\ell-k)-1}}\left(\frac{\Delta_{k}^{\prime}(v)}{\Delta_{k}(v)}\right)^{B_{\ell-1}}, \\
\left(\frac{\Delta_{\ell}^{\prime}(v)}{\Delta_{\ell}(v)}\right)^{B_{\ell}} & =\frac{1}{y_{\ell, 2}},
\end{aligned}
$$

где $n_{k}=2(\ell+1-k), k=2, \ldots, \ell, u \prod_{n=M}^{N} a_{n}=1$ nрu $N<M$. 
Теперь матричные элементы можно найти, разрешая указанные выше рекурсивные соотношения.

Лемма 2.6. Пусть $v$ определено как в (1.67) и (1.68). Тогда имеют место следующие выражения матричных элементов $v$ через переменные $y_{i, k}$ :

$$
\begin{aligned}
\Delta_{\omega_{i}, s_{i}}(v)^{B_{\ell}} & =\sum_{n=i}^{\ell} y_{i, n}, \quad i=1, \ldots, \ell \\
\Delta_{1}(v)^{B_{\ell}}= & \prod_{n=1}^{\ell} y_{1, n} \cdot \prod_{k=2}^{n_{1}} \prod_{n=1}^{n_{k} / 2} y_{k, 2 n-1}, \\
\Delta_{k}(v)^{B_{\ell}}= & \prod_{n=2}^{n_{1}} y_{1, n}^{2} \cdot \prod_{i=k+1}^{\ell} \prod_{n=1}^{n_{i} / 2} y_{i, 2 n-1}^{2} \cdot \prod_{i=2}^{k} \prod_{n=1}^{n_{i} / 2} y_{i, 2 n-1} y_{i, 2 n}, \\
\left.\frac{\Delta_{1}^{\prime}(v)}{\Delta_{1}(v)}\right)^{B_{\ell}}= & \sum_{n=1}^{\ell} \frac{1}{y_{1, n}}\left(1+\frac{y_{2,2(n-1)}}{y_{2,2(n-1)-1}}\right) \prod_{i=n+1}^{\ell} \frac{y_{2,2(i-1)}}{y_{2,2(i-1)-1}}, \\
\left(\frac{\Delta_{k}^{\prime}(v)}{\Delta_{k}(v)}\right)^{B_{\ell}}= & \sum_{n=1}^{n_{k} / 2} \frac{1}{y_{k, 2 n}}\left(1+\frac{y_{k+1,2(n-1)}}{y_{k+1,2(n-1)-1}}\right) \prod_{i=n+1}^{n_{k} / 2} \frac{y_{k+1,2(i-1)}}{y_{k+1,2(i-1)-1}} \frac{y_{k, 2 i-1}}{y_{k, 2 i}}, \\
& k=2, \ldots, \ell,
\end{aligned}
$$

где $n_{1}=\ell u n_{k}=2(\ell+1-k)$.

Доказательство леммы 2.5 следует из непосредственной подстановки выражений из леммы 2.6 в (2.24) и (2.26). Аналогично, подставляя полученные выражения из леммы 2.6 в формулу (1.19) из предложения 1.1, мы получаем интегральную формулу (1.74), таким образом завершая доказательство теоремы 1.4 .

Рассмотрим интегральное представление для $\mathfrak{s o}_{2 \ell+1}$-функции Уиттекера в модифицированной факторизационной параметризации (1.89), (1.90). Начнем с аналога рекурсивных соотношений (2.39) для матричных элементов в модифицированной факторизационной параметризации. Для упрощения формулировки рекурсивных соотношений оказывается удобным рассматривать модифицированную версию

$$
\begin{aligned}
& y_{1,1}=e^{-x_{\ell, 1}} e^{x_{1,1}-z_{1,1}}, \quad y_{1, k}=e^{-x_{\ell, 1}}\left(e^{x_{k-1,1}-z_{k, 1}}+e^{x_{k, 1}-z_{k, 1}}\right), \\
& y_{k, 2 r-1}=e^{x_{\ell, k-1}-x_{\ell, k}} e^{z_{k+r-1, k}-x_{k+r-2, k-1}}, \\
& y_{k, 2 r}=e^{x_{\ell, k-1}-x_{\ell, k}} e^{z_{k+r-1, k}-x_{k+r-1, k-1}}
\end{aligned}
$$


при $k=2, \ldots, \ell$ и $r=1, \ldots, \ell+1-k$, модифицированной параметризации $(1.89)$ с учетом действия части $H_{R}$ образующих Картана (1.99).

ЛЕмма 2.7. Для унипотентного элемента общего вида $v \in N_{+}^{(0)}$ имеют место следующие выражения для матричных элементов $v$ через переменные $x_{k, i}, z_{k, i}$, определенные в $(2.45)$ :

$$
\begin{aligned}
& \frac{\Delta_{1}^{2}(v)}{\Delta_{2}(v)}=e^{x_{1,1}-2 z_{1,1}}, \\
& \frac{\Delta_{k}(v)}{\Delta_{k+1}(v)}=\exp \left\{-\sum_{i=1}^{k} x_{k, i}-2 z_{k, 1}+2 \sum_{i=2}^{k} z_{k, i}-\sum_{i=1}^{k-1} x_{k-1, i}\right\} \\
& \quad \times\left(e^{x_{k-1,1}}+e^{x_{k, 1}}\right)^{2}, \quad k=2, \ldots, \ell \\
& \Delta_{\ell+1}(v)=1 ; \\
& \frac{\Delta_{1}^{\prime}(v)}{\Delta_{1}(v)}=\sum_{k=1}^{\ell} e^{z_{k, 1}}, \quad \Delta_{n=k+1}^{\ell}(v) \\
& \left.\frac{\Delta_{k}^{\prime}(v)}{\Delta_{k}(v)} e^{x_{k, k}-z_{k, k}}+\sum_{n=2, \ldots, \ell .}^{x_{n-1, k}-z_{n, k}}+e^{x_{n, k}-z_{n, k}}\right), \quad k=2, \ldots,
\end{aligned}
$$

Здесъ $x_{\ell, k}=0, k=1, \ldots, \ell$. Мь полагаем, что члены вида $e^{z_{\ell+1, i}}$ в $(2.47)$ сокращаются и, как обычно, $\sum_{n=i}^{j} a_{n}=0$, если $i>j$.

Подставляя (2.46), (2.47) в (1.17), (1.18), мы получаем выражения для векторов Уиттекера в параметризации (2.45). Полагая $x_{\ell, k}=0, k=1, \ldots, \ell$, мы приходим к выражениям для векторов Уиттекера из леммы 1.6. Для доказательства теоремы 1.6 остается учесть вид меры $d \mu_{N_{+}}$в модифицированной факторизационной параметризации. Это завершает доказательство теоремы 1.6.

2.3.3. Выражения для $\mathfrak{s p}_{2 \ell}$-матричных элементов: доказательства теоремы 1.8 и теоремы 1.10. В этом пункте мы находим выражения для матричных элементов, возникающих в конструкции интегральных представлений $\mathfrak{s p}_{2 \ell^{-}}$ функций Уиттекера, используя факторизационную параметризацию открытого подмножества в $N_{+} \subset \mathrm{Sp}(2 \ell)$. Это дает доказательство интегральных представлений для $\mathfrak{s p}_{2 \ell}$-функций Уиттекера, анонсированных в части 1.

Мы используем корневые данные для $\mathfrak{g}=\mathfrak{s p}_{2 \ell}$, определенные в (1.131). Разложение $w_{0}=s_{i_{1}} s_{i_{2}} \cdots s_{i_{m}}, m=m_{\ell}$, элемента максимальной длины $w_{0} \in W\left(C_{\ell}\right)$, отвечающее приведенному слову $I_{\ell}=\left(i_{1}, \ldots, i_{m_{\ell}}\right)$, определяет следующее упорядочение положительных кокорней: $R_{+}^{\vee}=\left\{\gamma_{k}^{\vee}=s_{i_{1}} \cdots s_{i_{k-1}} \alpha_{k}^{\vee}, k=1, \ldots, m_{\ell}\right\}$. Для системы корней типа $C_{\ell}$ мы имеем $m_{\ell}=\ell^{2}$. Мы рассматриваем разложение элемента $w_{0}$, описываемое следующим приведенным словом:

$$
I_{\ell}=(1,212, \ldots,(\ell \ldots 212 \ldots \ell))
$$


Соответствующее упорядочение на положительных кокорнях имеет вид:

$$
\begin{aligned}
& \gamma_{(\ell-1)^{2}+1}^{\vee}=2 \alpha_{1}^{\vee}+\cdots+2 \alpha_{\ell-1}^{\vee}+\alpha_{\ell}^{\vee}, \\
& \gamma_{2}^{\vee}=2 \alpha_{1}^{\vee}+\alpha_{2}^{\vee}, \\
& \gamma_{1}^{\vee}=\alpha_{1}^{\vee} \quad \gamma_{3}^{\vee}=\alpha_{1}^{\vee}+\alpha_{2}^{\vee} \text {, } \\
& \gamma_{\ell(\ell-1)}^{\vee}=2 \alpha_{1}^{\vee}+\alpha_{2}^{\vee}+\cdots+\alpha_{\ell}^{\vee}, \\
& \gamma_{4}^{\vee}=\alpha_{2}^{\vee} \text {, } \\
& \gamma_{(\ell-1)^{2}+\ell}^{\vee}=\alpha_{1}^{\vee}+\cdots+\alpha_{\ell}^{\vee} \text {, } \\
& \gamma_{\ell^{2}}^{\vee}=\alpha_{\ell}^{\vee}
\end{aligned}
$$

Рекурсивная параметризация открытого подмножества $N_{+}^{(0)}$ в $N_{+}$, определяемая приведенным словом $I_{\ell}$, имеет следующий вид. Для $v^{C_{\ell}} \in N_{+}^{(0)}$ мы получаем

$$
v^{C_{\ell}}(y)=\mathfrak{X}_{1}(y) \mathfrak{X}_{2}(y) \cdots \mathfrak{X}_{\ell}(y),
$$

где

$$
\mathfrak{X}_{k}(y)=X_{k}\left(y_{k, n_{k, k}-1}\right) \cdots X_{2}\left(y_{2, n_{k, 2}-1}\right) X_{1}\left(y_{1, n_{k, 1}}\right) X_{2}\left(y_{2, n_{k, 2}}\right) \cdots X_{k}\left(y_{k, n_{k, k}}\right)
$$

и $\mathfrak{X}_{1}=X_{1}\left(y_{1,1}\right)$. Для любого $k \in\{1, \ldots, \ell\}$ рассмотрим подслово

$$
I_{k}=\left(i_{1}, \ldots, i_{m_{k}}\right) \subset I_{\ell}=\left(i_{1}, \ldots, i_{m_{k}}, i_{m_{k}+1}, \ldots, i_{m_{\ell}}\right)
$$

с $\left|I_{k}\right|=m_{k}=k^{2}$. Пусть $C_{k}$ - соответствующая подсистема корней в $R_{+}$и $v^{C_{k}}=\mathfrak{X}_{1} \cdots \mathfrak{X}_{k}$ - факторизационная параметризация на соответствующей подгруппе. Параметры факторизации для $v(y)^{C_{k}}$ естественно занумеровать как $\left\{y_{i, n} ; 1 \leqslant i \leqslant k, 1 \leqslant n \leqslant n_{k, i}\right\}$, где

$$
n_{k, 1}=k, \quad n_{k, i}=2(k+1-i), \quad 1<i \leqslant \ell .
$$

Обозначим также $n_{i}:=n_{\ell, i}$.

Мы интересуемся явными выражениями для матричных элементов в терминах параметров факторизации $\left\{y_{i, n}\right\}$ :

$$
\Delta_{i}(v):=\left\langle\xi_{\omega_{i}}^{-}\left|\pi_{i}\left(v \dot{w}_{0}^{-1}\right)\right| \xi_{\omega_{i}}^{+}\right\rangle, \quad \Delta_{i}^{\prime}(v):=\left\langle\xi_{\omega_{i}}^{-}\left|\pi_{i}\left(v f_{i^{*}} \dot{w}_{0}^{-1}\right)\right| \xi_{\omega_{i}}^{+}\right\rangle,
$$

где $\pi_{i}=\pi_{\omega_{i}}-$ фундаментальное представление со старшим весом $\omega_{i}$, а $\xi_{\omega_{i}}^{+}$и $\xi_{\omega_{i}}^{-}-$старший и младший векторы в представлении $\pi_{i}$ такие, что $\left\langle\xi_{\omega_{i}}^{+} \mid \xi_{\omega_{i}}^{-}\right\rangle=1$. Заметим, что для алгебры Ли $\mathfrak{s p}_{2 \ell}$ инволюция $i \rightarrow i^{*}$, определенная в $(2.5)$, тривиальна, т. е. $i^{*}=i$. Доказательство следующего утверждения получается последовательным вычислением матричных элементов с учетом соотношений Серра и определяющих идеалов для фундаментальных представлений с использованием техники работы [16]. 
Лемма 2.8. Матричные элементы $\Delta_{i}(v)^{C_{\ell}}$ u $\Delta_{i}^{\prime}(v)^{C_{\ell}}$ удовлетворяют следующим рекурсивным соотношениям:

$$
\begin{aligned}
\Delta_{i}(v)^{C_{\ell}}= & \left(y_{1, n_{1}} \prod_{k=2}^{i} y_{k, n_{k}-1} y_{k, n_{k}} \prod_{k=i+1}^{\ell} y_{k, n_{k}-1}^{2}\right) \Delta_{i}(v)^{C_{\ell-1}}, \quad i=1, \ldots, \ell, \\
\left(\frac{\Delta_{1}^{\prime}(v)}{\Delta_{1}(v)}\right)^{C_{\ell}}= & \frac{1}{y_{1, \ell}}\left(1+\frac{y_{2,2(\ell-1)}}{y_{2,2(\ell-1)-1}}\right)^{2}+\left(\frac{y_{2,2(\ell-1)}}{y_{2,2(\ell-1)-1}}\right)^{2}\left(\frac{\Delta_{1}^{\prime}(v)}{\Delta_{1}(v)}\right)^{C_{\ell-1}}, \\
\left(\frac{\Delta_{k}^{\prime}(v)}{\Delta_{k}(v)}\right)^{C_{\ell}}= & \frac{1}{y_{k, 2(\ell+1-k)}}\left(1+\frac{y_{k+1,2(\ell-k)}}{y_{k+1,2(\ell-k)-1}}\right) \\
& +\frac{y_{k, 2(\ell+1-k)-1}}{y_{k, 2(\ell+1-k)}} \frac{y_{k+1,2(\ell-k)}}{y_{k+1,2(\ell-k)-1}}\left(\frac{\Delta_{k}^{\prime}(v)}{\Delta_{k}(v)}\right)^{C_{\ell-1}}, \quad k=2, \ldots, \ell-1, \\
\left(\frac{\Delta_{\ell}^{\prime}(v)}{\Delta_{\ell}(v)}\right)^{C_{\ell}}= & \frac{1}{y_{\ell, 2}} .
\end{aligned}
$$

Теперь матричные элементы можно найти, разрешая рекурсивные соотношения (2.51).

Лемма 2.9. Пусть $v$ определен как в (1.134) и (1.135). Тогда имеют место следующие соотношения для матричных элементов $v$ в терминах переменных $y_{i, k}$ :

$$
\begin{aligned}
\Delta_{\omega_{i}, \dot{s}_{i}}(v)^{C_{\ell}} & =\sum_{n=i}^{\ell} y_{i, n}, \quad i=1, \ldots, \ell \\
\Delta_{\omega_{i}, \dot{w}_{0}^{-1}}(v)^{C_{\ell}} & =\prod_{n=1}^{n_{1}} y_{1, n} \cdot \prod_{k=2}^{i} \prod_{n=1}^{n_{k}} y_{k, n} \cdot \prod_{k=i+1}^{\ell} \prod_{n=1}^{n_{k} / 2} y_{k, 2 n-1}^{2}, \\
\left(\frac{\Delta_{1}^{\prime}(v)}{\Delta_{1}(v)}\right)^{C_{\ell}} & =\sum_{n=1}^{n_{1}} \frac{1}{y_{1, n}}\left(1+\frac{y_{2,2(n-1)}}{y_{2,2(n-1)-1}}\right)^{2-1} \prod_{i=n}^{\ell}\left(\frac{y_{2,2 i}}{y_{2,2 i-1}}\right)^{2}, \\
\left(\frac{\Delta_{k}^{\prime}(v)}{\Delta_{k}(v)}\right)^{C_{\ell}} & =\sum_{n=1}^{n_{k} / 2} \frac{1}{y_{k, 2 n}}\left(1+\frac{y_{k+1,2(n-1)}}{y_{k+1,2(n-1)-1}}\right) \\
\left(\frac{\Delta_{\ell}^{\prime}(v)}{\Delta_{\ell}(v)}\right)^{C_{\ell}} & =\frac{1}{y_{\ell, 2}} .
\end{aligned}
$$

Комбинируя эти выражения с выражениями (1.21) для инвариантной меры на $N_{+}$и подставляя в $(1.17),(1.18)$ и $(1.19)$, мы завершаем доказательство теоремы 1.8 .

Теперь рассмотрим интегральное представление для $\mathfrak{s p}_{2 \ell}$-функции Уиттекера в модифицированной факторизационной параметризации (1.150), (1.151). Начнем с аналога рекурсивных соотношений (2.51) для матричных элементов 
в модифицированной факторизационной параметризации. Для упрощения вида рекурсивных соотношений оказывается полезной скрученная версия модифицированной параметризации (1.152) с учетом действия части $H_{R}(1.162)$ элемента максимального тора $e^{-h_{z}}$. Тем самым, мы рассматриваем следующую замену переменных:

$$
\begin{gathered}
y_{1,1}=e^{-2 z_{\ell, 1}} e^{x_{1,1}+z_{1,1}}, \quad y_{1, k}=e^{-2 z_{\ell, 1}}\left(e^{z_{k-1,1}+x_{k, 1}}+e^{z_{k, 1}+x_{k, 1}}\right), \\
y_{k, 2 r-1}=e^{z_{\ell, k-1}-z_{\ell, k}} e^{x_{k+r-1, k}-z_{k+r-2, k-1}}, \\
y_{k, 2 r}=e^{z_{\ell, k-1}-z_{\ell, k}} e^{x_{k+r-1, k}-z_{k+r-1, k-1}}
\end{gathered}
$$

здесь $k=2, \ldots, \ell, r=1, \ldots, \ell+1-k$.

ЛЕмма 2.10. 1) В модифицированной факторизационной параметризации рекурсивные соотношения (2.51) имеют вид

$$
\left(\frac{\Delta_{k}^{\prime}}{\Delta_{k}}\right)^{C_{n}}=e^{z_{n-1, k}-x_{n, k}}+e^{z_{n, k}-x_{n, k}}+\frac{e^{\left\langle\alpha_{k}, \underline{z}_{n-1}\right\rangle}}{e^{\left\langle\alpha_{k}, \underline{z}_{n}\right\rangle}}\left(\frac{\Delta_{k}^{\prime}}{\Delta_{k}}\right)^{C_{n-1}}, \quad 1 \leqslant k<n<\ell,
$$

с решением

$$
\left(\frac{\Delta_{k}^{\prime}(v)}{\Delta_{k}(v)}\right)^{C_{n}}=e^{z_{k, k}-x_{k, k}}+\sum_{n=k+1}^{\ell}\left(e^{z_{n-1, k}-x_{n, k}}+e^{z_{n, k}-x_{n, k}}\right), \quad k=1, \ldots, \ell,
$$

где $\underline{z}_{n}=\left(z_{n, 1}, \ldots, z_{n, n}\right)$ и мы полагаем $\left\langle\alpha_{k}, \underline{z}_{n}\right\rangle=z_{n, k+1}-z_{n, k},\left\langle\alpha_{k}, \underline{z}_{n-1}\right\rangle=$ $z_{n-1, k+1}-z_{n-1, k}$.

2) Имеют место следующие выражения для $\Delta_{k}(v)$ через переменные $x_{k, i}, z_{k, i}$ :

$$
\begin{aligned}
\left(\frac{\Delta_{1}(v)}{\Delta_{2}(v)}\right)^{C_{n}}= & e^{-z_{\ell, 1}} e^{x_{1,1}} \\
\left(\frac{\Delta_{k}(v)}{\Delta_{k+1}(v)}\right)^{C_{n}}= & e^{-z_{\ell, k}}\left(e^{z_{k, 1}}+e^{z_{k-1,1}}\right) \\
& \quad \times \exp \left\{-\sum_{i=1}^{k} z_{k, i}+x_{k, 1}+2 \sum_{i=2}^{k} x_{k, i}-\sum_{i=1}^{k-1} z_{k-1, i}\right\},
\end{aligned}
$$

где $k=2, \ldots, \ell u \Delta_{\ell+1}=1$.

Подставляя (2.53), (2.54) в (1.17), (1.18), мы получаем левый и правый векторы Уиттекера в параметризации (2.53). Полагая $z_{\ell, k}=0, k=1, \ldots, \ell$, мы приходим к формулам для векторов Уиттекера из леммы 1.8. Для доказательства теоремы 1.10 остается учесть вид меры $d \mu_{N_{+}}$в модифицированной факторизационной параметризации. Это завершает доказательство теоремы 1.10.

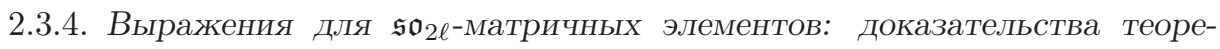
мы 1.12 и теоремы 1.14. В этом пункте мы находим выражения для матричных элементов, возникающих в конструкции интегральных представлений $\mathfrak{s o}_{2 \ell}$-функций Уиттекера, используя факторизационную параметризацию открытого подмножества в $N_{+} \subset \mathrm{SO}(2 \ell)$. Это дает доказательство интегральных представлений для $\mathfrak{s o}_{2 \ell}$-функций Уиттекера, анонсированных в части 1 . 
Мы используем корневые данные, описанные в (1.192). Приведенное разложение $w_{0}=s_{i_{1}} s_{i_{2}} \cdots s_{i_{m_{\ell}}}$ элемента максимальной длины $w_{0} \in W\left(D_{\ell}\right)$, отвечающее приведенному слову $I_{\ell}=\left(i_{1}, \ldots, i_{m_{\ell}}\right)$ с $m_{\ell}=\ell(\ell-1)$, определяет следующее упорядочение положительных кокорней: $R_{+}^{\vee}=\left\{\gamma_{k}^{\vee}=s_{i_{1}} \cdots s_{i_{k-1}} \alpha_{k}^{\vee}\right.$, $\left.k=1, \ldots, m_{\ell}\right\}$ для $\mathfrak{s p}_{2 \ell}$. Мы рассматриваем разложение элемента $w_{0}$, описываемое следующим приведенным словом:

$$
I_{\ell}=(12,3123, \ldots,(\ell \ldots 3123 \ldots \ell)) .
$$

Соответствующее упорядочение положительных кокорней имеет вид

$$
\begin{aligned}
& \gamma_{3}^{\vee}=\alpha_{1}^{\vee}+\alpha_{2}^{\vee}+\alpha_{3}^{\vee}, \\
& \gamma_{1}^{\vee}=\alpha_{1}^{\vee}, \quad \gamma_{4}^{\vee}=\alpha_{2}^{\vee}+\alpha_{3}^{\vee}, \\
& \gamma_{2}^{\vee}=\alpha_{2}^{\vee}, \quad \gamma_{5}^{\vee}=\alpha_{1}^{\vee}+\alpha_{3}^{\vee} \text {, } \\
& \gamma_{6}^{\vee}=\alpha_{3}^{\vee} \\
& \gamma_{m_{\ell-1}+1}^{\vee}=\alpha_{1}^{\vee}+\alpha_{2}^{\vee}+2\left(\alpha_{3}^{\vee}+\cdots+\alpha_{\ell-1}^{\vee}\right)+\alpha_{\ell}^{\vee}, \\
& \gamma_{m_{\ell-1}+2}^{\vee}=\alpha_{1}^{\vee}+\alpha_{2}^{\vee}+2\left(\alpha_{3}^{\vee}+\cdots+\alpha_{\ell-2}^{\vee}\right)+\alpha_{\ell-1}^{\vee}+\alpha_{\ell}^{\vee} \text {, } \\
& \gamma_{m_{\ell-1}+\ell-2}^{\vee}=\alpha_{1}^{\vee}+\alpha_{2}^{\vee}+\alpha_{3}^{\vee}+\cdots+\alpha_{\ell}^{\vee}, \\
& \gamma_{m_{\ell-1}+\ell-1}^{\vee}=p_{\ell-1} \alpha_{1}^{\vee}+p_{\ell} \alpha_{2}^{\vee}+\alpha_{3}^{\vee}+\cdots+\alpha_{\ell}^{\vee}, \\
& \gamma_{m_{\ell-1}+\ell}^{\vee}=p_{\ell} \alpha_{1}^{\vee}+p_{\ell+1} \alpha_{2}^{\vee}+\alpha_{3}^{\vee}+\cdots+\alpha_{\ell}^{\vee}, \\
& \gamma_{m_{\ell-1}+\ell+1}^{\vee}=\alpha_{3}^{\vee}+\cdots+\alpha_{\ell}^{\vee} \text {, } \\
& \gamma_{m_{\ell}}^{\vee}=\alpha_{\ell}^{\vee} .
\end{aligned}
$$

Рекурсивная параметризация открытого подмножества $N_{+}^{(0)}$ в $N_{+}$, соответствующая приведенному слову $I_{\ell}$, приобретает следующий вид. Для $v^{D_{\ell}} \in N_{+}^{(0)}$ мы имеем

$$
v^{D_{\ell}}(y)=\mathfrak{X}_{2}(y) \mathfrak{X}_{2}(y) \cdots \mathfrak{X}_{\ell}(y),
$$

где

$$
\begin{aligned}
\mathfrak{X}_{k}(y)= & X_{k}\left(y_{k, n_{k, k}-1}\right) \cdots X_{3}\left(y_{2, n_{k, 3}-1}\right) X_{1}\left(y_{1, n_{k, 1}}\right) X_{2}\left(y_{1, n_{k, 2}}\right) \\
& \times X_{3}\left(y_{2, n_{k, 3}}\right) \cdots X_{k}\left(y_{k, n_{k, k}}\right)
\end{aligned}
$$

и $\mathfrak{X}_{2}=X_{1}\left(y_{1,1}\right) X_{2}\left(y_{2,1}\right)$. Для любого $k \in\{1, \ldots, \ell\}$ рассмотрим подслово

$$
I_{k}=\left(i_{1}, \ldots, i_{m_{k}}\right) \subset I_{\ell}=\left(i_{1}, \ldots, i_{m_{k}}, i_{m_{k}+1}, \ldots, i_{m_{\ell}}\right)
$$

с $\left|I_{k}\right|=m_{k}=k(k-1)$. Пусть $D_{k}$ - соответствующая подсистема корней в $R_{+}$ и $v^{D_{k}}=\mathfrak{X}_{2} \cdots \mathfrak{X}_{k}$ - факторизационная параметризация на соответствующей подгруппе. Параметры факторизации для $v^{D_{k}}(y)$ естественно занумеровать как $\left\{y_{i, n} ; 1 \leqslant i \leqslant k, 1 \leqslant n \leqslant n_{k, i}\right\}$, где

$$
n_{k, 1}=n_{k, 2}=k-1, \quad n_{k, i}=2(k+1-i), \quad 2<i \leqslant \ell .
$$


Также мы обозначаем $n_{i}=n_{\ell, i}$.

Мы интересуемся явными выражениями для матричных элементов в терминах параметров факторизации $\left\{y_{i, n}\right\}$ :

$$
\Delta_{i}(v):=\left\langle\xi_{\omega_{i}}^{-}\left|\pi_{i}\left(v \dot{w}_{0}^{-1}\right)\right| \xi_{\omega_{i}}^{+}\right\rangle, \quad \Delta_{i}^{\prime}(v):=\left\langle\xi_{\omega_{i}}^{-}\left|\pi_{i}\left(v f_{i^{*}} \dot{w}_{0}^{-1}\right)\right| \xi_{\omega_{i}}^{+}\right\rangle
$$

где $\pi_{i}=\pi_{\omega_{i}}$ - фундаментальное представление со старшим весом $\omega_{i}$, a $\xi_{\omega_{i}}^{+}$ и $\xi_{\omega_{i}}^{-}-$старший и младший векторы в представлении $\pi_{i}$ такие, что $\left\langle\xi_{\omega_{i}}^{+} \mid \xi_{\omega_{i}}^{-}\right\rangle=1$. Заметим, что в случае алгебры Ли $\mathfrak{s o}_{2 \ell}$ мы имеем $i \rightarrow i^{*}$ для инволюции, определенной в (2.5). Доказательство следующего утверждения получается последовательным вычислением матричных элементов с учетом соотношений Серра и определяющих идеалов для фундаментальных представлений с использованием техники работы [16].

Лемма 2.11. Имеют место следующие рекурсивные соотношения:

$$
\begin{aligned}
\Delta_{1}(v)^{D_{\ell}} & =\left(\left(y_{1, n_{1}}\right)^{p_{\ell-1}}\left(y_{2, n_{2}}\right)^{p_{\ell}} \prod_{k=3}^{\ell} y_{k, n_{k}-1}\right) \Delta_{1}(v)^{D_{\ell-1}}, \\
\Delta_{2}(v)^{D_{\ell}} & =\left(\left(y_{1, n_{1}}\right)^{p_{\ell}}\left(y_{2, n_{2}}\right)^{p_{\ell+1}} \prod_{k=3}^{\ell} y_{k, n_{k}-1}\right) \Delta_{2}(v)^{D_{\ell-1},} \\
\Delta_{i}(v)^{D_{\ell}} & =\left(y_{1, n_{1}} y_{2, n_{2}} \prod_{k=3}^{i} y_{k, n_{k}-1} y_{k, n_{k}} \prod_{k=i+1}^{\ell} y_{k, n_{k}-1}^{2}\right)_{i}(v)^{D_{\ell-1}}, \quad 2<i<\ell, \\
\left(\frac{\Delta_{1}^{\prime}}{\Delta_{1}}\right)^{D_{2 r}} & =\frac{1}{y_{1,2 r-1}}\left(1+\frac{y_{3,2(2 r-2)}}{y_{3,2(2 r-2)-1}}\right)+\frac{y_{2,2 r-1}}{y_{1,2 r-1}} \frac{y_{3,2(2 r-2)}}{y_{3,2(2 r-2)-1}}\left(\frac{\Delta_{1}^{\prime}}{\Delta_{1}}\right)^{D_{2 r-1},}, \\
\left(\frac{\Delta_{1}^{\prime}}{\Delta_{1}}\right)^{D_{2 r+1}} & =\frac{1}{y_{2,2 r}}\left(1+\frac{y_{3,2(2 r-1)}}{y_{3,2(2 r-1)-1}}\right)+\frac{y_{1,2 r}}{y_{2,2 r}} \frac{y_{3,2(2 r-1)}}{y_{3,2(2 r-1)-1}}\left(\frac{\Delta_{1}^{\prime}}{\Delta_{1}}\right)^{D_{2 r}}, \quad, \quad 2.58, \\
\left(\frac{\Delta_{2}^{\prime}}{\Delta_{2}}\right)^{D_{2 r}} & =\frac{1}{y_{2,2 r-1}}\left(1+\frac{y_{3,4(r-1)}}{y_{3,4(r-1)-1}}\right)+\frac{y_{1,2 r-1}}{y_{2,2 r-1}} \frac{y_{3,4(r-1)}}{y_{3,4(r-1)-1}}\left(\frac{\Delta_{2}^{\prime}}{\Delta_{2}}\right)^{D_{2 r-1}}, \\
\left(\frac{\Delta_{2}^{\prime}}{\Delta_{2}}\right)^{D_{2 r+1}} & =\frac{1}{y_{1,2 r-1}}\left(1+\frac{y_{3,2(2 r-1)}}{y_{3,2(2 r-1)-1}}\right)+\frac{y_{2,2 r}}{y_{1,2 r}} \frac{y_{3,2(2 r-1)}}{y_{3,2(2 r-1)-1}}\left(\frac{\Delta_{2}^{\prime}}{\Delta_{2}}\right)^{D_{2 r}} .
\end{aligned}
$$

Матричные элементы могут быть вычислены как решения рекурсивных уравнений. Результаты вычислений представлены в следующей лемме.

Лемма 2.12. Пусть $v$ определен как в (2.56). Тогда имеют место следующие выражения матричных элементов $v$ через переменные $y_{i, k}$ :

$$
\begin{aligned}
& \Delta_{i}(v)=\prod_{n=1}^{\ell-1} y_{i, n}^{\varpi(n)} y_{i^{*}, n}^{1-\varpi(n)} \prod_{n=1}^{(\ell-1) / 2} y_{2,2 n} \prod_{n=3}^{\ell} \prod_{i=3}^{n} y_{i, 2(n+1-i)-1}, \quad i=1,2 ; \\
& \Delta_{k}(v)=\prod_{n=k}^{\ell}\left(y_{1, n-1} y_{2, n-1} \prod_{j=3}^{k} y_{j, 2(n-j)+1} y_{j, 2(n-j)+2} \prod_{m=k+1}^{n} y_{m, 2(n-m)-1}^{2}\right)
\end{aligned}
$$


при $k=3, \ldots, \ell$, где $\varpi(n)=\left(1-(-1)^{n}\right) / 2$ - четность числа $n$, и $n_{k}=2(\ell+1-k)$, $2<k \leqslant \ell$

$$
\begin{aligned}
\left(\frac{\Delta_{1}^{\prime}}{\Delta_{1}}+\frac{\Delta_{2}^{\prime}}{\Delta_{2}}\right)^{D_{\ell}}=\sum_{n=1}^{\ell-1} & {\left[\frac{1}{y_{1, \ell-1}} \prod_{k=1}^{n-1}\left(\frac{y_{1, \ell-k}}{y_{1, \ell-k-1}}\right)^{1-\varpi(k)}\left(\frac{y_{2, \ell-k}}{y_{2, \ell-k-1}}\right)^{\varpi(k)}\right.} \\
& \left.+\frac{1}{y_{2, \ell-1}} \prod_{k=1}^{n-1}\left(\frac{y_{1, \ell-k}}{y_{1, \ell-k-1}}\right)^{\varpi(k)}\left(\frac{y_{2, \ell-k}}{y_{2, \ell-k-1}}\right)^{1-\varpi(k)}\right] \\
& \times\left(1+\frac{y_{3,2(\ell-n-1)}}{y_{3,2(\ell-n-1)-1}}\right)^{1-\delta_{n, \ell-1}} \prod_{k=1}^{n-1} \frac{y_{3,2(\ell-k-1)}}{y_{3,2(\ell-k-1)-1}} \\
\left(\frac{\Delta_{k}^{\prime}(v)}{\Delta_{k}(v)}\right)^{D_{\ell}}= & \frac{1}{y_{k, 2(\ell+1-k)}} \sum_{n=k}^{\ell}\left(1+\frac{y_{k+1,2(\ell-n)}}{y_{k+1,2(\ell-n)-1}}\right)^{1-\delta_{n, \ell}} \\
& \times \prod_{j=k}^{n-1} \frac{y_{k, 2(\ell-j)-1}}{y_{k, 2(\ell-j)}} \frac{y_{k+1,2(\ell-j)}}{y_{k+1,2(\ell-j)-1}}
\end{aligned}
$$

nри $k=3, \ldots, \ell$.

Для доказательства леммы 1.9 нужно подставить полученные выражения для матричных элементов из леммы 2.12 в (2.24) и (2.26). Утверждение теоремы 1.12 (интегральная формула (1.205)) следует из непосредственной подстановки выражений из леммы 2.12 в формулу (1.19) из предложения 1.1.

Теперь рассмотрим интегральные представления для $\mathfrak{s o}_{2 \ell}$-функции Уиттекера в модифицированной факторизационной параметризации (1.217). Начнем с аналога рекурсивных соотношений (2.58) для матричных элементов в модифицированной факторизационной параметризации. Для упрощения вида рекурсивных соотношений оказывается полезным рассматривать скрученную версию модифицированной параметризации (1.217), учитывая действие части $e^{H_{R}}(1.226)$ элемента максимального тора $e^{-h_{x}}$. Тем самым, мы рассматриваем следующую замену переменных:

$$
\begin{aligned}
y_{1, n} & =e^{x_{\ell, 1}-x_{\ell, 2}}\left(e^{z_{n, 1}-x_{n, 1}}+e^{z_{n, 1}-x_{n+1,1}}\right), & & n=1, \ldots, \ell-1, \\
y_{2, n} & =e^{-x_{\ell, 1}-x_{\ell, 2}}\left(e^{z_{n, 1}+x_{n, 1}}+e^{z_{n, 1}+x_{n+1,1}}\right), & & n=1, \ldots, \ell-1, \\
y_{k, 2 r-1} & =e^{x_{\ell, k-1}-x_{\ell, k}} e^{z_{k+r-2, k-1}-x_{k+r-2, k-1}}, & & k=3, \ldots, \ell, \\
y_{k, 2 r} & =e^{x_{\ell, k-1}-x_{\ell, k}} e^{z_{k+r-2, k-1}-x_{k+r-1, k-1}}, & & r=1, \ldots, \ell+1-k .
\end{aligned}
$$

Лемма 2.13. Имеет место следующее рекурсивное соотношение относительно переменных, определенных в (2.62):

$$
\begin{aligned}
\left(\frac{\Delta_{1}^{\prime}}{\Delta_{1}}\right)^{D_{n}}+\left(\frac{\Delta_{2}^{\prime}}{\Delta_{2}}\right)^{D_{n}}=e^{x_{n-1,2}-z_{n-1,1}}+e^{x_{n, 2}-z_{n-1,1}} & \\
& +\frac{e^{x_{n-1,2}+(-1)^{\varpi(n)} x_{n-1,1}}}{e^{x_{n, 2}+(-1)^{\varpi(n)} x_{n, 1}}\left(\frac{\Delta_{1}^{\prime}}{\Delta_{1}}\right)^{D_{n-1}}} \\
& +\frac{e^{x_{n-1,1}+(-1)^{1-\varpi(n)} x_{n-1,2}}}{e^{x_{n, 1}+(-1)^{1-\varpi(n)} x_{n, 2}}}\left(\frac{\Delta_{2}^{\prime}}{\Delta_{2}}\right)^{D_{n-1}}
\end{aligned}
$$

где $\varpi(n)=\left(1-(-1)^{n}\right) / 2$ - четность числа $n=3, \ldots, \ell$. 
Решая рекурсивные уравнения, несложно получить следующий результат.

Лемма 2.14. Для унипотентного элемента $v \in N_{+}$имеют место следующие выражения для матричных элементов $v$ в модифицированной параметризачии:

$$
\begin{aligned}
& \frac{\Delta_{1}^{\prime}}{\Delta_{1}}+\frac{\Delta_{2}^{\prime}}{\Delta_{2}}=\sum_{k=1}^{\ell-1}\left(e^{z_{k, k}-x_{k, k-1}}+e^{z_{k, k}-x_{k+1, k}}\right), \\
& \frac{\Delta_{k}^{\prime}}{\Delta_{k}}=e^{z_{\ell-1, \ell+1-k}-x_{\ell, \ell+1-k}}, \quad k=3, \ldots, l \\
& \frac{\Delta_{2}}{\Delta_{1}}=e^{-x_{\ell, 1}} e^{x_{1,1}} \\
& \frac{\Delta_{1} \Delta_{2}}{\Delta_{3}}=e^{-x_{\ell, 2}} \exp \left\{-\left(x_{2,1}+x_{2,2}\right)+2 z_{1,1}-x_{1,1}\right\}\left(e^{x_{1,1}}+e^{x_{2,1}}\right)^{2}, \\
& \frac{\Delta_{k}}{\Delta_{k+1}}=e^{-x_{\ell, k} \exp \left\{-\sum_{i=1}^{k} x_{k, i}+2 \sum_{i=1}^{k-1} z_{k-1, i}-\sum_{i=1}^{k-1} x_{k-1, i}\right\}} \\
& \quad \times\left(e^{x_{k-1,1}}+e^{x_{k, 1}}\right)^{2}, \quad k=3, \ldots, \ell
\end{aligned}
$$

$u \Delta_{\ell+1}=1$.

Подставляя (2.64) в (1.17), (1.18), мы получаем векторы Уиттекера в параметризации (2.62). Полагая $x_{\ell, k}=0, k=1, \ldots, \ell$, мы приходим к выражениям для векторов Уиттекера из леммы 1.10. Для завершения доказательства теоремы 1.14 остается учесть вид меры $d \mu_{N_{+}}$в модифицированной факторизационной параметризации.

2.4. Реализация $\mathscr{U}(\mathfrak{g})$ дифференциальными операторами. В этом разделе мы доказываем формулы для реализации образующих Шевалле классических алгебр Ли типов $X_{\ell}$ для $X=A, B, C, D$ дифференциальными операторами, действующими в пространстве эквивариантных функций на группе $N_{+}$ относительно факторизационной параметризации. Аналогичные формулы для реализации образующих алгебры Ли в модифицированной факторизационной параметризации (представление Гаусса-Гивенталя) можно получить непосредственно, используя замену переменных, которую мы обсуждали в части 1 ; здесь мы не будем на этом останавливаться.

Начнем с изложения общей стратегии получения реализации алгебры Ли дифференциальными операторами. Пусть $V_{\mu}$ - пространство $B_{-}$-эквивариантных функций на $B_{-} \backslash G$ :

$$
f(b g)=\chi_{\mu}(b) f(g), \quad b \in B_{-},
$$

где $\chi_{\mu}$ - характер борелевской подгруппы $B_{-} \subset G$. Представление основной серии алгебры $\mathscr{U}(\mathfrak{g})$ в пространстве $V_{\mu}$ определяется как

$$
(X f)(v)=\left.\frac{\partial}{\partial \varepsilon} f\left(v e^{\varepsilon X}\right)\right|_{\varepsilon \rightarrow 0}, \quad X \in \mathfrak{g} .
$$

Пусть $I_{\ell}=\left(i_{1}, \ldots, i_{m_{\ell}}\right)$ - приведенное слово, отвечающее приведенному разложению $w_{0}=s_{i_{1}} \cdots s_{i_{\ell}}$ элемента $w_{0}$ максимальной длины из группы Вейля 
$W\left(X_{\ell}\right)$ (здесь $X_{\ell}$ принадлежит классическим сериям $A, B, C$ или $D$ и имеет ранг $\ell$ ). При этом можно выбрать слово $I_{\ell}$ так, что оно обладает рекурсивной структурой по отношению к рангу $\ell$ алгебры Ли типа $X_{\ell}$. Далее рассмотрим рекурсивную факторизационную параметризацию унипотентных элементов из соответствующей классической группы Ли $G$ типа $X_{\ell}$ :

$$
v^{X_{\ell}}=\mathfrak{X}_{X_{1}}^{X_{2}} \cdots \mathfrak{X}_{X_{\ell-1}}^{X_{\ell}}=v^{X_{\ell-1}} \mathfrak{X}_{X_{\ell-1}}^{X_{\ell}} .
$$

Для алгебры Ли $\mathfrak{g}$ классического типа $X_{\ell}$ мы получим явные формулы, определяющие представление $\pi_{\mu}$ алгебры $\mathscr{U}(\mathfrak{g})$ в пространстве $V_{\mu}$, в два шага. На первом шаге мы используем рекурсивную структуру (2.67), чтобы построить рекурсивные соотношения между образующими Шевалле, действующими в представлении $\left(\pi_{\mu}, V_{\mu}\right)$, для алгебр Ли одного типа, ранги которых отличаются на 1. На втором шаге мы решаем рекурсивные соотношения между образами $\pi_{\mu}\left(e_{i}\right), \pi_{\mu}\left(f_{i}\right), \pi_{\mu}\left(h_{i}\right)$ образующих Шевалле алгебр типов $X_{\ell}$ и $X_{\ell \pm 1}$ и получаем явные формулы для действия образующих в пространстве $V_{\mu}$.

Начнем со списка соотношений между однопараметрическими подгруппами в $G$ (см., например, [14], [16]). Пусть $e_{i}, h_{i}, f_{i}, i=1, \ldots, \ell$, - образующие Шевалле в $\mathfrak{g}$ и $A=\left\|a_{j, i}\right\|$ - матрица Картана. Введем однопараметрические подгруппы:

$$
X_{i}(y)=e^{y f_{i}}, \quad \alpha_{i}^{\vee}(1+y)=e^{y h_{i}}, \quad Y_{i}(y)=e^{y e_{i}}, \quad i=1, \ldots, \ell .
$$

Тогда выполнены соотношения

$$
\begin{aligned}
X_{i}(y) \alpha_{j}^{\vee}(1+\varepsilon) & =\alpha_{j}^{\vee}(1+\varepsilon) X_{i}\left(y+a_{j i} \varepsilon y\right) \bmod \varepsilon^{2}, \\
X_{i}(y) Y_{i}(\varepsilon) & =Y_{i}(\varepsilon) \alpha_{i}^{\vee}(1-\varepsilon y) X_{i}\left(y-\varepsilon y^{2}\right) \bmod \varepsilon^{2} .
\end{aligned}
$$

При $a_{i, j}=a_{j, i}=-1$ мы получаем

$$
X_{i}\left(y_{1}\right) X_{j}\left(y_{2}\right) X_{i}(\varepsilon)=X_{j}\left(\varepsilon \frac{y_{2}}{y_{1}}\right) X_{i}\left(y_{1}+\varepsilon\right) X_{j}\left(y_{2}-\varepsilon \frac{y_{2}}{y_{1}}\right) \bmod \varepsilon^{2} .
$$

При $a_{i, j}=-2$ и $a_{j, i}=-1$ имеем

$$
\begin{aligned}
& X_{j}\left(y_{1}\right) X_{i}\left(y_{2}\right) X_{j}\left(y_{3}\right) X_{i}(\varepsilon)=X_{i}\left(\varepsilon \frac{y_{3}}{y_{1}}\right) X_{j}\left(y_{1}+2 \varepsilon \frac{y_{3}}{y_{2}}\right) X_{i}\left(y_{2}+\varepsilon-\varepsilon \frac{y_{3}}{y_{1}}\right) \\
& \times X_{j}\left(y_{3}-2 \varepsilon \frac{y_{3}}{y_{2}}\right) \bmod \varepsilon^{2} .
\end{aligned}
$$

При $a_{j, i}=-2$ и $a_{i, j}=-1$ имеем

$$
\begin{gathered}
X_{j}\left(y_{1}\right) X_{i}\left(y_{2}\right) X_{j}\left(y_{3}\right) X_{i}(\varepsilon)=X_{i}\left(\varepsilon \frac{y_{3}^{2}}{y_{1}^{2}}\right) X_{j}\left(y_{1}+\varepsilon \frac{y_{1} y_{3}+y_{3}^{2}}{y_{1} y_{2}}\right) X_{i}\left(y_{2}+\varepsilon \frac{y_{1}^{2}-y_{3}^{2}}{y_{1}^{2}}\right) \\
\times X_{j}\left(y_{3}-\varepsilon \frac{y_{1} y_{3}+y_{3}^{2}}{y_{1} y_{2}}\right) \bmod \varepsilon^{2} .
\end{gathered}
$$

Для вывода рекурсивного соотношения между образующими алгебры Ли рассмотрим правое действие однопараметрических подгрупп (2.68) на рекурсивной 
факторизационной параметризации (2.67) элемента $v^{\ell} \in N_{+}^{(0)}$. Воспользуемся соотношениями (2.69)-(2.73), чтобы сдвинуть образующие на один шаг влево. Например, в случае однопараметрической подгруппы, порожденной $f_{i}$, мы имеем:

$$
v^{(\ell)} X_{i}(\varepsilon)=v^{(\ell-1)} \mathfrak{X}_{\ell-1}^{\ell}(y) X_{i}(\varepsilon)=v^{(\ell-1)} X_{i}\left(c_{i}(y) \varepsilon\right) \mathfrak{X}_{\ell-1}^{\ell}\left(y^{\prime}(y)\right) \bmod \varepsilon^{2}
$$

Это приводит к рекурсивным соотношениям между образующими алгебры Ли ранга $\ell$ через образующие алгебры Ли ранга $(\ell-1)$ и дифференциальные операторы по переменным $y_{i, n}$, параметризующим $\mathfrak{X}_{\ell-1}^{\ell}$. На последнем шаге редукции мы используем (2.65). В следующих пунктах мы приводим рекурсивные соотношения и явные формулы для образующих всех классических алгебр Ли без дальнейших комментариев.

2.4.1. Образующие $\mathfrak{g l}_{\ell+1}$ : доказательство предложения 1.2. Пусть $E_{i, i+1}^{(\ell+1)}$, $E_{i, i}^{(\ell+1)}, E_{i+1, i}^{(\ell+1)}$ - образы базисных элементов $e_{i, j}$ алгебры $\mathfrak{g l}_{\ell+1}$, действующих в представлении основной серии $\left(\pi_{\mu}, V_{\mu}\right)$. Ниже мы приводим рекурсивные соотношения и явные выражения для этих образующих.

Рекурсивные соотношения имеют вид

$$
\begin{aligned}
E_{i, i+1}^{(\ell+1)}= & \frac{\partial}{\partial y_{i, \ell+1-i}}+\frac{y_{i-1, \ell+2-i}}{y_{i, \ell+1-i}}\left(E_{i-1, i}^{(\ell)}-\frac{\partial}{\partial y_{i-1, \ell+2-i}}\right), \\
E_{i, i}^{(\ell+1)}= & \mu_{i}^{(\ell+1)}-\mu_{i}^{(\ell)}+E_{i, i}^{(\ell)}+y_{i-1, \ell+2-i} \frac{\partial}{\partial y_{i-1, \ell+2-i}} \\
& \quad-y_{i, \ell+1-i} \frac{\partial}{\partial y_{i, \ell+1-i}}, \quad i \neq \ell+1, \\
E_{\ell+1, \ell+1}^{(\ell+1)}= & \mu_{\ell+1}^{(\ell+1)}+y_{\ell, 1} \frac{\partial}{\partial y_{\ell, 1}}, \\
E_{i+1, i}^{(\ell+1)}= & E_{i+1, i}^{(\ell)}+y_{i, \ell+1-i}\left(E_{i, i}^{(\ell)}-E_{i+1, i+1}^{(\ell)}\right) \\
& \quad-y_{i, \ell+1-i}\left\{y_{i, \ell+1-i} \frac{\partial}{\partial y_{i, \ell+1-i}}-y_{i+1, \ell-i} \frac{\partial}{\partial y_{i+1, \ell-i}}\right\} .
\end{aligned}
$$

Разрешая рекурсию, мы приходим к (1.34). Это завершает доказательство предложения 1.2 .

2.4.2. Образующие $\mathfrak{s o}_{2 \ell+1}$ : доказательство предложения 1.4. Пусть $E_{i}^{(\ell)}$, $H_{i}^{(\ell)}, F_{i}^{(\ell)}$ - образы базисных элементов Шевалле алгебры Ли $\mathfrak{s o}_{2 \ell+1}$, действующих в представлении основной серии $\left(\pi_{\mu}, V_{\mu}\right)$. Ниже мы приводим рекурсивные соотношения и явные выражения для этих образующих.

Рекурсивные соотношения имеют вид

$$
\begin{aligned}
F_{1}^{(\ell)}= & \frac{\partial}{\partial y_{1, \ell}}+\frac{y_{2,2(\ell-1)}}{y_{2,2(\ell-1)-1}}\left(F_{1}^{(\ell-1)}-\frac{\partial}{\partial y_{1, \ell}}\right) \\
& +2 \frac{y_{2,2(\ell-1)}}{y_{1, \ell}}\left\{\frac{\partial}{\partial y_{2,2(\ell-1)-1}}-\frac{\partial}{\partial y_{2,2(\ell-1)}}\right\},
\end{aligned}
$$




$$
\begin{aligned}
& F_{k}^{(\ell)}=\frac{\partial}{\partial y_{k, 2(\ell+1-k)}}+\frac{y_{k, 2(\ell+1-k)-1}}{y_{k, 2(\ell+1-k)}} \frac{y_{k+1,2(\ell-k)}}{y_{k+1,2(\ell-k)-1}}\left(F_{k}^{(\ell-1)}-\frac{\partial}{\partial y_{k, 2(\ell+1-k)-1}}\right) \\
& +\frac{y_{k+1,2(\ell-k)}}{y_{k, 2(\ell+1-k)}}\left\{\frac{\partial}{\partial y_{k+1,2(\ell-k)-1}}-\frac{\partial}{\partial y_{k+1,2(\ell-k)}}\right\}, \quad k=2, \ldots, \ell-1, \\
& E_{1}^{(\ell)}=E_{1}^{(\ell-1)}-y_{1, \ell} H_{1}^{(\ell-1)}+2 y_{1, \ell} y_{2,2(\ell-1)-1} \frac{\partial}{\partial y_{2,2(\ell-1)-1}}-y_{1, \ell}^{2} \frac{\partial}{\partial y_{1, \ell}}, \\
& E_{2}^{(\ell)}=E_{2}^{(\ell-1)}-\left(y_{2,2(\ell-1)-1}+y_{2,2(\ell-1)}\right) H_{2}^{(\ell-1)} \\
& +\left(y_{2,2(\ell-1)-1}+y_{2,2(\ell-1)}\right) y_{3,2(\ell-2)-1} \frac{\partial}{\partial y_{3,2(\ell-2)-1}}+2 y_{2,2(\ell-1)} y_{1, \ell} \frac{\partial}{\partial y_{1, \ell}} \\
& -\left\{y_{2,2(\ell-1)-1}^{2} \frac{\partial}{\partial y_{2,2(\ell-1)-1}}+2 y_{2,2(\ell-1)} y_{2,2(\ell-1)-1} \frac{\partial}{\partial y_{2,2(\ell-1)-1}}\right. \\
& \left.+y_{2,2(\ell-1)}^{2} \frac{\partial}{\partial y_{2,2(\ell-1)}}\right\} \\
& E_{k}^{(\ell)}=E_{k}^{(\ell-1)}-\left(y_{k, 2(\ell+1-k)-1}+y_{k, 2(\ell+1-k)}\right) H_{k}^{(\ell-1)} \\
& +\left(y_{k, 2(\ell+1-k)-1}+y_{k, 2(\ell+1-k)}\right) y_{k+1,2(\ell-k)-1} \frac{\partial}{\partial y_{k+1,2(\ell-k)-1}} \\
& +y_{k, 2(\ell+1-k)}\left\{y_{k-1,2(\ell+2-k)-1} \frac{\partial}{\partial y_{k-1,2(\ell+2-k)-1}}\right. \\
& \left.+y_{k-1,2(\ell+2-k)} \frac{\partial}{\partial y_{k-1,2(\ell+2-k)}}\right\}-\left\{y_{k, 2(\ell+1-k)-1}^{2} \frac{\partial}{\partial y_{k, 2(\ell+1-k)-1}}\right. \\
& +2 y_{k, 2(\ell+1-k)-1} y_{k, 2(\ell+1-k)} \frac{\partial}{\partial y_{k, 2(\ell+1-k)-1}} \\
& \left.+y_{k, 2(\ell+1-k)}^{2} \frac{\partial}{\partial y_{k, 2(\ell+1-k)}}\right\} \text {. }
\end{aligned}
$$

Для действия образующих картановской подалгебры мы получаем

$$
H_{k}^{(\ell)}=\left\langle\mu^{(\ell)}, \alpha_{k}^{\vee}\right\rangle+\sum_{i=1}^{\ell} a_{k, i} \sum_{j=1}^{n_{i}} y_{i, j} \frac{\partial}{\partial y_{i, j}}, \quad k=1, \ldots, \ell
$$

где $n_{1}=\ell, n_{k}=2(\ell+1-k)$ при $1<k \leqslant \ell$.

Разрешая рекурсию, мы приходим к (1.69), (1.70) и (1.71). Это завершает доказательство предложения 1.4.

2.4.3. Образующие $\mathfrak{s p}_{2 \ell}$ : доказательство предложения 1.8. Пусть $E_{i}^{(\ell)}, H_{i}^{(\ell)}$, $F_{i}^{(\ell)}$ - образы базисных элементов Шевалле алгебры Ли $\mathfrak{s p}_{2 \ell}$, действующих в представлении основной серии $\left(\pi_{\mu}, V_{\mu}\right)$. Ниже мы приводим рекурсивные соотношения и явные выражения для этих образующих. 
Рекурсивные соотношения имеют вид

$$
\begin{aligned}
& F_{1}^{(\ell)}=\frac{\partial}{\partial y_{1, \ell}}+\left(\frac{y_{2,2(\ell-1)}}{y_{2,2(\ell-1)-1}}\right)^{2}\left(F_{1}^{(\ell-1)}-\frac{\partial}{\partial y_{1, \ell}}\right) \\
& +\frac{y_{2,2(\ell-1)-1} y_{2,2(\ell-1)}+y_{2,2(\ell-1)}^{2}}{y_{1, \ell} y_{2,2(\ell-1)-1}}\left\{\frac{\partial}{\partial y_{2,2(\ell-1)-1}}-\frac{\partial}{\partial y_{2,2(\ell-1)}}\right\}, \\
& F_{k}^{(\ell)}=\frac{\partial}{\partial y_{k, 2(\ell+1-k)}}+\frac{y_{k, 2(\ell+1-k)-1}}{y_{k, 2(\ell+1-k)}} \frac{y_{k+1,2(\ell-k)}}{y_{k+1,2(\ell-k)-1}}\left(F_{k}^{(\ell-1)}-\frac{\partial}{\partial y_{k, 2(\ell+1-k)-1}}\right) \\
& +\frac{y_{k+1,2(\ell-k)}}{y_{k, 2(\ell+1-k)}}\left\{\frac{\partial}{\partial y_{k+1,2(\ell-k)-1}}-\frac{\partial}{\partial y_{k+1,2(\ell-k)}}\right\}, \quad 1<k<\ell, \\
& F_{\ell}^{(\ell)}=\frac{\partial}{\partial y_{\ell, 2}} \\
& E_{1}^{(\ell)}=E_{1}^{(\ell-1)}-y_{1, \ell} H_{1}^{(\ell-1)}+y_{1, \ell} y_{2,2(\ell-1)-1} \frac{\partial}{\partial y_{2,2(\ell-1)-1}}-y_{1, \ell}^{2} \frac{\partial}{\partial y_{1, \ell}}, \\
& E_{2}^{(\ell)}=E_{2}^{(\ell-1)}-\left(y_{2,2(\ell-1)-1}+y_{2,2(\ell-1)}\right) H_{2}^{(\ell-1)}+2 y_{2,2(\ell-1)-1} y_{1, \ell} \frac{\partial}{\partial y_{1, \ell}} \\
& +\left(y_{2,2(\ell-1)-1}+y_{2,2(\ell-1)}\right) y_{3,2(\ell-2)-1} \frac{\partial}{\partial y_{3,2(\ell-2)-1}} \\
& -\left\{y_{2,2(\ell-1)-1}^{2} \frac{\partial}{\partial y_{2,2(\ell-1)-1}}+2 y_{2,2(\ell-1)-1} y_{2,2(\ell-1)-1} \frac{\partial}{\partial y_{2,2(\ell-1)-1}}\right. \\
& \left.+y_{2,2(\ell-1)}^{2} \frac{\partial}{\partial y_{2,2(\ell-1)}}\right\} \\
& E_{k}^{(\ell)}=E_{k}^{(\ell-1)}-\left(y_{k, 2(\ell+1-k)-1}+y_{k, 2(\ell+1-k)}\right) H_{k}^{(\ell-1)} \\
& +\left(y_{k, 2(\ell+1-k)-1}+y_{k, 2(\ell+1-k)}\right) y_{k+1,2(\ell-k)-1} \frac{\partial}{\partial y_{k+1,2(\ell-k)-1}} \\
& +y_{k, 2(\ell+1-k)}\left\{y_{k-1,2(\ell+2-k)-1} \frac{\partial}{\partial y_{k-1,2(\ell+2-k)-1}}\right. \\
& \left.+y_{k-1,2(\ell+2-k)} \frac{\partial}{\partial y_{k-1,2(\ell+2-k)}}\right\}-\left\{y_{k, 2(\ell+1-k)-1}^{2} \frac{\partial}{\partial y_{k, 2(\ell+1-k)-1}}\right. \\
& +2 y_{k, 2(\ell+1-k)-1} y_{k, 2(\ell+1-k)} \frac{\partial}{\partial y_{k, 2(\ell+1-k)-1}} \\
& \left.+y_{k, 2(\ell+1-k)}^{2} \frac{\partial}{\partial y_{k, 2(\ell+1-k)}}\right\}, \quad 2<k<\ell \\
& E_{\ell}^{(\ell)}=\left(y_{\ell, 1}+y_{\ell, 2}\right)\left\{-\left\langle\mu, \alpha_{\ell}^{\vee}\right\rangle+y_{\ell-1,1} \frac{\partial}{\partial y_{\ell-1,1}}+y_{\ell-1,2} \frac{\partial}{\partial y_{\ell-1,2}}\right\} \\
& +y_{\ell, 2}\left\{y_{\ell-1,3} \frac{\partial}{\partial y_{\ell-1,3}}+y_{\ell-1,4} \frac{\partial}{\partial y_{\ell-1,4}}\right\} \\
& -\left\{y_{\ell, 1}^{2} \frac{\partial}{\partial y_{\ell, 1}}+2 y_{\ell, 1} y_{\ell, 2} \frac{\partial}{\partial y_{\ell, 1}}+y_{\ell, 2}^{2} \frac{\partial}{\partial y_{\ell, 2}}\right\} \text {. }
\end{aligned}
$$


Для действия образующих подалгебры Картана мы имеем:

$$
H_{k}^{(\ell)}=\left\langle\mu, \alpha_{k}^{\vee}\right\rangle+\sum_{i=1}^{\ell} a_{k, i} \sum_{j=1}^{n_{i}} y_{i, j} \frac{\partial}{\partial y_{i, j}}, \quad k=1, \ldots, \ell
$$

где $n_{1}=\ell$ и $n_{k}=2(\ell+1-k)$ при $1<k \leqslant \ell$.

Разрешая рекурсию, мы получаем выражения (1.136), (1.137) и (1.138). Это завершает доказательство предложения 1.8.

2.4.4. Образующие $\mathfrak{s o}_{2 \ell}$ : доказательство предложения 1.12. Пусть $E_{i}^{(\ell)}, H_{i}^{(\ell)}$, $F_{i}^{(\ell)}$ - образы базисных элементов Шевалле алгебры Ли $\mathfrak{s o}_{2 \ell}$, действующих в представлении основной серии $\left(\pi_{\mu}, V_{\mu}\right)$. Ниже мы приводим рекурсивные соотношения и явные выражения для этих образующих.

Рекурсивные соотношения имеют вид

$$
\begin{aligned}
& F_{1}^{(\ell)}=\frac{\partial}{\partial y_{1, \ell-1}}+\frac{y_{2, \ell-1}}{y_{1, \ell-1}} \frac{y_{3,2(\ell-2)}}{y_{3,2(\ell-2)-1}}\left(F_{2}^{(\ell-1)}-\frac{\partial}{\partial y_{2, \ell-1}}\right) \\
& +\frac{y_{3,2(\ell-2)}}{y_{1, \ell-1}}\left\{\frac{\partial}{\partial y_{3,2(\ell-2)-1}}-\frac{\partial}{\partial y_{3,2(\ell-2)}}\right\} \text {, } \\
& F_{2}^{(\ell)}=\frac{\partial}{\partial y_{2, \ell-1}}+\frac{y_{1, \ell-1}}{y_{2, \ell-1}} \frac{y_{3,2(\ell-2)}}{y_{3,2(\ell-2)-1}}\left(F_{1}^{(\ell-1)}-\frac{\partial}{\partial y_{1, \ell-1}}\right) \\
& +\frac{y_{3,2(\ell-2)}}{y_{2, \ell-1}}\left\{\frac{\partial}{\partial y_{3,2(\ell-2)-1}}-\frac{\partial}{\partial y_{3,2(\ell-2)}}\right\} \text {, } \\
& F_{k}^{(\ell)}=\frac{\partial}{\partial y_{k, 2(\ell+1-k)}}+\frac{y_{k, 2(\ell+1-k)-1}}{y_{k, 2(\ell+1-k)}} \frac{y_{k+1,2(\ell-k)}}{y_{k+1,2(\ell-k)-1}}\left(F_{k}^{(\ell-1)}-\frac{\partial}{\partial y_{k, 2(\ell+1-k)-1}}\right) \\
& +\frac{y_{k+1,2(\ell-k)}}{y_{k, 2(\ell+1-k)}}\left\{\frac{\partial}{\partial y_{k, 2(\ell+1-k)-1}}-\frac{\partial}{\partial y_{k, 2(\ell+1-k)}}\right\}, \quad k=3, \ldots, \ell-1, \\
& E_{i}^{(\ell)}=E_{i}^{(\ell-1)}-y_{i, \ell-1}^{2} \frac{\partial}{\partial y_{i, \ell-1}} \\
& +y_{i, \ell-1}\left\{-H_{i}^{(\ell-1)}+y_{3,2(\ell-2)-1} \frac{\partial}{\partial y_{3,2(\ell-2)-1}}\right\}, \quad i=1,2, \\
& E_{3}^{(\ell)}=E_{3}^{(\ell-1)}-\left\{y_{3,2(\ell-2)-1}^{2} \frac{\partial}{\partial y_{3,2(\ell-2)-1}}+y_{3,2(\ell-2)}^{2} \frac{\partial}{\partial y_{3,2(\ell-2)}}\right\} \\
& +\left(y_{3,2(\ell-2)-1}+y_{3,2(\ell-2)}\right)\left\{-H_{3}^{(\ell-1)}+y_{4,2(\ell-3)-1} \frac{\partial}{\partial y_{4,2(\ell-3)-1}}\right\} \\
& +y_{3,2(\ell-2)}\left\{y_{1, \ell-1} \frac{\partial}{\partial y_{1, \ell-1}}+y_{2, \ell-1} \frac{\partial}{\partial y_{2, \ell-1}}\right\} \text {, } \\
& E_{k}^{(\ell)}=E_{k}^{(\ell-1)}-\left\{y_{k, 2(\ell+1-k)-1}^{2} \frac{\partial}{\partial y_{k, 2(\ell+1-k)-1}}+y_{k, 2(\ell+1-k)}^{2} \frac{\partial}{\partial y_{k, 2(\ell+1-k)}}\right\} \\
& +\left(y_{k, 2(\ell+1-k)-1}+y_{k, 2(\ell+1-k)}\right)
\end{aligned}
$$




$$
\begin{aligned}
& \times\left\{-H_{k}^{(\ell-1)}+y_{k+1,2(\ell-k)-1} \frac{\partial}{\partial y_{k+1,2(\ell-k)-1}}\right\} \\
& +y_{k, 2(\ell+1-k)}\left\{y_{k-1,2(\ell+2-k)-1} \frac{\partial}{\partial y_{k-1,2(\ell+2-k)-1}}\right. \\
& \left.+y_{k-1,2(\ell+2-k)} \frac{\partial}{\partial y_{k-1,2(\ell+2-k)}}\right\}, \quad 3<k \leqslant \ell .
\end{aligned}
$$

Для действия образующих подалгебры Картана мы имеем:

$$
H_{i}^{(\ell)}=\left\langle\mu, \alpha_{i}^{\vee}\right\rangle+\sum_{k=1}^{\ell} a_{i, k} \sum_{j=1}^{n_{k}} y_{k, j} \frac{\partial}{\partial y_{k, j}}, \quad k=1, \ldots, \ell .
$$

где $n_{1}=n_{2}=\ell-1, n_{k}=2(\ell+1-k)$ при $2<k \leqslant \ell$.

Разрешая рекурсию, мы получаем выражения (1.197)-(1.202). Это завершает доказательство предложения 1.12.

\section{Список литературы}

[1] A. Givental, "Stationary phase integrals, quantum Toda lattices, flag manifolds and the mirror conjecture", Topics in singularity theory, Amer. Math. Soc. Transl. Ser. 2, 180, Amer. Math. Soc., Providence, RI, 1997, 103-115; arXiv: alg-geom/9612001.

[2] D. Joe, B. Kim, "Equivariant mirrors and the Virasoro conjecture for flag manifolds", Int. Math. Res. Not., 2003:15 (2003), 859-882; arXiv: math.AG/0210377.

[3] И. М. Гельфанд, М. С. Цейтлин, "Конечномерные представления группы унимодулярных матриц", Докл. АН СССР, 71:5 (1950), 825-828.

[4] V.V. Batyrev, "Toric degenerations of Fano varieties and constructing mirror manifolds", The Fano Conference, Univ. Torino, Turin, 2004, 109-122; arXiv: alg-geom/9712034.

[5] V. V. Batyrev, I. Ciocan-Fontanine, B. Kim, D. van Straten, "Mirror symmetry and toric degenerations of partial flag manifolds", Acta Math., 184:1 (2000), 1-39; arXiv: math.AG/9803108.

[6] A. Gerasimov, S. Kharchev, D. Lebedev, S. Oblezin, "On a Gauss-Givental representation of quantum Toda chain wave function", Int. Math. Res. Not., 2006 (2006), Art. ID 96489, 23 pp.; arXiv: math.RT/0505310.

[7] B. Kostant, "Quantization and representation theory", Representation theory of Lie groups, Proceedings of the SRC/LMS Research Symposium (Oxford, 1977), London Math. Soc. Lecture Note Ser., 34, Cambridge University Press, Cambridge-New York, 1979, 287-316.

[8] B. Kostant, "On Whittaker vectors and representation theory", Invent. Math., 1978, № 2, 101-184.

[9] E. T. Whittaker, G. N. Watson, A course of modern analysis, 4th ed., Cambridge Univ. Press, Cambridge, 1996, 608 pp.; pус. пер.: Э. Т. Уиттекер, Дж. Н. Ватсон, Курс современного анализа, Ч. 2. Трансцендентные функиии, 2-е изд., Мир, М., $1963,500 \mathrm{c}$.

[10] V. Pasquier, M. Gaudin, "The periodic Toda chain and a matrix generalization of the Bessel function recursion relation", J. Phys. A, 25:20 (1992), 5243-5252.

[11] E. K. Sklyanin, "Bäcklund transformations and Baxter's $Q$-operator", Integrable systems: from classical to quantum (Montréal, QC, 1999), CRM Proc. Lecture Notes, 26, Amer. Math. Soc., Providence, RI, 2000, 227-250. 
[12] K. Rietsch, "A mirror construction for the totally nonnegative part of the Peterson variety", Nagoya Math. J., 183 (2006), 105-142; arXiv: math.AG/0604170.

[13] М. А. Ольшанецкий, А. М. Переломов, А. Г. Рейман, М. А. Семенов-ТянШанский, "Интегрируемые системы. II", Динамические системы - 7, Итоги науки и техники. Соврем. пробл. матем. Фундам. напр., 16, ВИНИТИ, М., 1987, 86-226; англ. пер.: M. A. Ol'shanetskij, M. A. Perelomov, A. G. Reyman, M. A. Semenov-Tian-Shansky, "Integrable systems. II", Dynamical systems. VII. Integrable systems, nonholonomic dynamical systems, Encyclopaedia Math. Sci., 16, Springer-Verlag, Berlin, 1994, 83-259.

[14] G. Lusztig, "Total positivity in reductive groups", Lie theory and geometry, Progr. Math., 123, Birkhäuser Boston, Boston, MA, 1994, 531-568.

[15] S. Fomin, A. Zelevinsky, "Double Bruhat cells and total positivity", J. Amer. Math. Soc., 12:2 (1999), 335-380; arXiv: math.RT/9802056.

[16] A. Berenstein, A. Zelevinsky, "Total positivity in Schubert varieties", Comment. Math. Helv., 72:1 (1997), 128-166.

[17] В.Г. Дринфельд, В.В. Соколов, “Алгебры Ли и уравнения типа Кортевегаде Фриза", Итоги науки и техники. Соврем. пробл. матем. Нов. достиж., 24, ВИНИТИ, М., 1984, 81-180; англ. пер.: V. G. Drinfeld, V. V. Sokolov, "Lie algebras and equations of Korteweg-de Vries type", J. Soviet Math., 30:2 (1985), 1975-2036.

[18] A.D. Berenstein, A. V. Zelevinsky, "Tensor product multiplicities and convex polytopes in partition space", J. Geom. Phys., 5:3 (1989), 453-472.

[19] V. Lakshmibai, "Degeneration of flag varieties to toric varieties", C. R. Acad. Sci. Paris Sér. I Math., 321:9 (1995), 1229-1234.

[20] A. Gerasimov, D. Lebedev, S. Oblezin, On a Gauss-Givental representation for classical groups, arXiv: math.RT/0608152.

[21] A. Gerasimov, D. Lebedev, S. Oblezin, Baxter Q-operator and Givental integral representation for $C_{n}$ and $D_{n}$, arXiv: math.RT/0609082.

[22] A. Gerasimov, D. Lebedev, S. Oblezin, "Quantum Toda chains intertwined", Алгебра и анализ, 22:3 (2010), 107-141; англ. изд.: A. Gerasimov, D. Lebedev, S. Oblezin, "Quantum Toda chains intertwined", St. Petersburg Math. J., 22:3 (2011), 411-435.

[23] P. Etingof, Differential topology, infinite-dimensional Lie algebras, and applications, Amer. Math. Soc. Transl. Ser. 2, 194, Amer. Math. Soc., Providence, RI, 1999, 9-25; arXiv: math.QA/9901053.

[24] V.G. Kac, Infinite-dimensional Lie algebras, 3rd ed., Cambridge University Press, Cambridge, 1990, xхіi+400 pp.; рус. пер.: В. Кац, Бесконечномерные алгебры Ли, Мир, М., 1993, 426 с.

[25] T.A. Springer, "Reductive groups", Automorphic forms, representations and L-functions, Part 1 (Oregon State Univ., Corvallis, OR, 1977), Proc. Sympos. Pure Math., 33, eds. A. Borel, W. Casselman, Amer. Math. Soc., Providence, RI, 1979, $3-27$.

[26] S. Helgason, Differential geometry, Lie groups, and symmetric spaces, Pure Appl. Math., 34, Academic Press, New York-London, 1978, xv+628 pp.; pyc. пер. издания 1962 г.: С. Хелгасон, Дифференииальная геометрия и симметрические пространства, Мир, М., 1964, 533 с.

[27] H. Jacquet, "Fonctions de Whittaker associées aux groupes de Chevalley", Bull. Soc. Math. France, 95 (1967), 243-309.

[28] M. Hashizume, "Whittaker functions on semisimple Lie groups", Hiroshima Math. J., 12:2 (1982), 259-293.

[29] A. Gerasimov, S. Kharchev, A. Morozov, M. Olshanetsky, A. Marshakov, A. Mironov, "Liouville type models in the group theory framework. I. Finite-dimensional algebras", Internat. J. Modern Phys. A, 12:14 (1997), 2523-2583. 
[30] N. Bourbaki, Éléments de mathématique. Fasc. XXXIV, Groupes et algèbres de Lie: Chapitre VI: Systèmes des racines, Hermann, Paris, 1968; pус. пер.: Н. Бурбаки, Группы и алгебры Ли. Главы IV-VI, Мир, М., 1972, 334 с.

[31] R. J. Baxter, Exactly solved models in statistical mechanics, Academic Press, London, 1982, хіi+486 pр.; рус. пер.: Р. Бэкстер, Точно решаемъе модели в статистической механике, Мир, М., 1985, 488 с.

[32] А. Г. Рейман, М. А. Семенов-Тян-Шанский, "Интегрируемые системы", Теоретико-групповой подход, Современная математика, Ин-т компьютерных исследований, М.-Ижевск, 2003, 352 с.

[33] K. Rietsch, "A mirror symmetric construction of $q H_{T}^{*}(G / P)_{(q)}$ ", Adv. Math., 217:6 (2008), 2401-2442; arXiv: math.AG/0511124v2.

\section{А. А. Герасимов (А. А. Gerasimov)}

Институт теоретической и экспериментальной физики им. А. И. Алиханова;

The Hamilton Mathematics Institute, Trinity College

Dublin, Ireland

\section{Д. Р. Лебедев (D. R. Lebedev)}

Институт теоретической и экспериментальной физики им. А. И. Алиханова

\section{C. В. Облезин (S. V. Oblezin)}

Институт теоретической и экспериментальной

физики им. А. И. Алиханова

E-mail: Sergey.Oblezin@itep.ru
Поступила в редакцию

14.07.2011 